\title{
Zariski Decompositions on Arithmetic Surfaces
}

\author{
by
}

\author{
Atsushi MORIWAKI
}

\begin{abstract}
In this paper, we establish the Zariski decompositions of arithmetic $\mathbb{R}$-Cartier divisors of continuous type on arithmetic surfaces and investigate their properties. We also develop a general theory of arithmetic $\mathbb{R}$-Cartier divisors on arithmetic varieties.

2010 Mathematics Subject Classification: Primary 14G40; Secondary 11G50, 37P30. Keywords: Zariski decomposition, arithmetic variety, Arakelov geometry.

\section{Contents}

$0 \quad$ Introduction 799

1 Zariski decompositions in vector spaces 808

2 Green functions for $\mathbb{R}$-Cartier divisors 814

3 Gromov's inequality and distorsion functions for $\mathbb{R}$-Cartier divisors 826

4 Plurisubharmonic upper envelopes 832

5 Arithmetic $\mathbb{R}$-Cartier divisors 843

6 Positivity of arithmetic $\mathbb{R}$-Cartier divisors 854

$7 \quad$ Limit of nef arithmetic $\mathbb{R}$-Cartier divisors on arithmetic surfaces 870

$8 \sigma$-decompositions on arithmetic surfaces 875

9 Zariski decompositions and their properties on arithmetic surfaces 882

References 897
\end{abstract}

\section{$\S 0$. Introduction}

Let $S$ be a non-singular projective surface over an algebraically closed field and let $\operatorname{Div}(S)$ be the group of Cartier divisors on $S$. An element of $\operatorname{Div}(S) \otimes_{\mathbb{Z}} \mathbb{R}$ is called an $\mathbb{R}$-Cartier divisor on $S$. It is said to be effective if it is a linear combination of curves with non-negative real coefficients. The problem of the Zariski decomposition for an effective $\mathbb{R}$-Cartier divisor $D$ is to find a decomposition $D=P+N$ with the following properties:

Communicated by S. Mukai. Received February 9, 2011. Revised March 7, 2012

A. Moriwaki: Department of Mathematics, Faculty of Science, Kyoto University, Kyoto, 606-8502, Japan;

e-mail: moriwaki@math.kyoto-u.ac.jp

(C) 2012 Research Institute for Mathematical Sciences, Kyoto University. All rights reserved. 
(1) $P, N \in \operatorname{Div}(S) \otimes_{\mathbb{Z}} \mathbb{R}$.

(2) $P$ is nef, that is, $(P \cdot C) \geq 0$ for all reduced and irreducible curves $C$ on $S$.

(3) $N$ is effective.

(4) Assuming $N \neq 0$, let $N=c_{1} C_{1}+\cdots+c_{l} C_{l}$ be the decomposition such that $c_{1}, \ldots, c_{l} \in \mathbb{R}_{>0}$ and $C_{1}, \ldots, C_{l}$ are distinct reduced and irreducible curves on $S$. Then the following hold:

(4.1) $\left(P \cdot C_{i}\right)=0$ for all $i$.

(4.2) The $l \times l$ matrix given by $\left(\left(C_{i} \cdot C_{j}\right)\right)_{\substack{1 \leq i \leq l \\ 1 \leq j \leq l}}$ is negative definite.

In 1962, Zariski [24] established the decomposition in the case where $D \in \operatorname{Div}(S)$. By the recent work due to Bauer [1] (see also Section 1), $P$ is characterized by the greatest element in

$$
\left\{M \in \operatorname{Div}(S) \otimes_{\mathbb{Z}} \mathbb{R} \mid D-M \text { is effective and } M \text { is nef }\right\} .
$$

In this paper, we would like to consider an arithmetic analogue of the above problem on an arithmetic surface. In order to make the main theorem clear, we need to introduce some terminology.

\section{$\S 0.1$. Green functions for $\mathbb{R}-$ Cartier divisors}

Let $V$ be an equidimensional smooth projective variety over $\mathbb{C}$. An element of $\operatorname{Div}(V)_{\mathbb{R}}:=\operatorname{Div}(V) \otimes_{\mathbb{Z}} \mathbb{R}$ is called an $\mathbb{R}$-Cartier divisor on $V$. For an $\mathbb{R}$-Cartier divisor $D$ on $V$, we will introduce several types of Green functions for $D$. We set $D=a_{1} D_{1}+\cdots+a_{l} D_{l}$, where $a_{1}, \ldots, a_{l} \in \mathbb{R}$ and $D_{i}$ 's are reduced and irreducible divisors on $V$. Let $g: V \rightarrow \mathbb{R} \cup\{ \pm \infty\}$ be a locally integrable function on $V$. We say $g$ is a $D$-Green function of $C^{\infty}$-type (resp. a $D$-Green function of $C^{0}$-type) on $V$ if, for each point $x \in V$, there are a small open neighborhood $U_{x}$ of $x$, local equations $f_{1}, \ldots, f_{l}$ of $D_{1}, \ldots, D_{l}$ over $U_{x}$ respectively and a $C^{\infty}$-function (resp. continuous function) $u_{x}$ over $U_{x}$ such that

$$
g=u_{x}+\sum_{i=1}^{l}\left(-a_{i}\right) \log \left|f_{i}\right|^{2} \quad \text { (a.e.) }
$$

on $U_{x}$. These definitions correspond $C^{\infty}$-metrics and continuous metrics. Moreover, it is necessary to introduce a degenerate version of semipositive metrics. We say $g$ is a $D$-Green function of $\mathrm{PSH}_{\mathbb{R}}$-type on $V$ if the above $u_{x}$ is a real valued plurisubharmonic function on $U_{x}$ (i.e., $u_{x}$ is a plurisubharmonic function on $U_{x}$ and $u_{x}(y) \in \mathbb{R}$ for all $\left.y \in U_{x}\right)$. More generally, let $\mathcal{L}_{\text {loc }}^{1}$ be the sheaf of locally integrable functions, that is,

$$
\mathcal{L}_{\text {loc }}^{1}(U)=\{g: U \rightarrow \mathbb{R} \cup\{ \pm \infty\} \mid g \text { is locally integrable }\}
$$


for an open set $U$ of $V$, and let us fix a subsheaf $\mathscr{T}$ of $\mathcal{L}_{\text {loc }}^{1}$ satisfying the following conditions (in (1)-(3), $U$ is an arbitrary open set of $V$ ):

(1) If $u, v \in \mathscr{T}(U)$ and $a \in \mathbb{R}_{\geq 0}$, then $u+v \in \mathscr{T}(U)$ and $a u \in \mathscr{T}(U)$.

(2) If $u, v \in \mathscr{T}(U)$ and $u \leq v$ almost everywhere, then $u \leq v$.

(3) If $\phi \in \mathscr{O}_{V}^{\times}(U)$ (i.e., $\phi$ is a nowhere vanishing holomorphic function on $U$ ), then $\log \left(|\phi|^{2}\right) \in \mathscr{T}(U)$.

Any such subsheaf $\mathscr{T}$ is called a type for Green functions on $V$. Moreover, $\mathscr{T}$ is said to be real valued if $u(x) \in \mathbb{R}$ for any open set $U, u \in \mathscr{T}(U)$ and $x \in U$. Using $\mathscr{T}$, we say $g$ is a $D$-Green function of $\mathscr{T}$-type on $V$ if the above $u_{x}$ is an element of $\mathscr{T}\left(U_{x}\right)$ for each $x \in V$. The set of all $D$-Green functions of $\mathscr{T}$-type on $V$ is denoted by $G_{\mathscr{T}}(V ; D)$. If $x \notin \operatorname{Supp}(D)$, then, by using $(2)$ and (3), we can see that the value

$$
u_{x}(x)+\sum_{i=1}^{l}\left(-a_{i}\right) \log \left|f_{i}(x)\right|^{2}
$$

does not depend on the choice of the local expression

$$
g=u_{x}+\sum_{i=1}^{l}\left(-a_{i}\right) \log \left|f_{i}\right|^{2} \quad \text { (a.e.) }
$$

this value is called the canonical value of $g$ at $x$ and denoted by $g_{\text {can }}(x)$. Note that $g_{\text {can }} \in \mathscr{T}(V \backslash \operatorname{Supp}(D))$ and $g=g_{\text {can }}$ (a.e.) on $V \backslash \operatorname{Supp}(D)$. Further, if $\mathscr{T}$ is real valued, then $g_{\text {can }}(x) \in \mathbb{R}$.

- $H^{0}(V, D)$ for an $\mathbb{R}$-Cartier divisor $D$ and its norm arising from a Green function. Let $D$ be an $\mathbb{R}$-Cartier divisor. If $V$ is connected, then $H^{0}(V, D)$ is defined by

$$
H^{0}(V, D):=\left\{\begin{array}{l|l}
\phi & \begin{array}{l}
\phi \text { is a non-zero rational function } \\
\text { on } V \text { with }(\phi)+D \geq 0
\end{array}
\end{array}\right\} \cup\{0\} .
$$

In general, let $V=V_{1} \cup \cdots \cup V_{r}$ be the decomposition of $V$ into connected components. Then

$$
H^{0}(V, D):=\bigoplus_{i=1}^{r} H^{0}\left(V_{i},\left.D\right|_{V_{i}}\right)
$$

Let $g$ be a $D$-Green function of $C^{0}$-type on $V$. For $\phi \in H^{0}(V, D)$, it is easy to see that $|\phi|_{g}:=\exp (-g / 2)|\phi|$ coincides with a continuous function almost everywhere on $V$, so that the supremum norm $\|\phi\|_{g}$ of $\phi$ with respect to $g$ is defined by

$$
\|\phi\|_{g}:=\operatorname{ess} \sup \left\{|\phi|_{g}(x) \mid x \in V\right\} .
$$




\section{§0.2. Arithmetic $\mathbb{R}-$ Cartier divisors}

Let $X$ be a $d$-dimensional generically smooth normal projective arithmetic variety, that is, $X$ is a flat and projective integral scheme over $\mathbb{Z}$ such that $X$ is normal, $X$ is smooth over $\mathbb{Q}$ and the Krull dimension of $X$ is $d$. Let $\operatorname{Div}(X)$ be the group of Cartier divisors on $X$. As before, an element of $\operatorname{Div}(X)_{\mathbb{R}}:=\operatorname{Div}(X) \otimes_{\mathbb{Z}} \mathbb{R}$ is called an $\mathbb{R}$-Cartier divisor on $X$. It is said to be effective if it is a linear combination of prime divisors with non-negative real coefficients. In addition, for $D, E \in \operatorname{Div}(X)_{\mathbb{R}}$, if $D-E$ is effective, then we write $D \geq E$ or $E \leq D$.

Let $D$ be an $\mathbb{R}$-Cartier divisor on $X$ and let $g$ be a locally integrable function on $X(\mathbb{C})$. A pair $\bar{D}=(D, g)$ is called an arithmetic $\mathbb{R}$-Cartier divisor on $X$ if $F_{\infty}^{*}(g)=g$ (a.e.), where $F_{\infty}$ is the complex conjugation map on $X(\mathbb{C})$. Moreover, $\bar{D}$ is said to be of $C^{\infty}$-type (resp. of $C^{0}$-type, of $\mathrm{PSH}_{\mathbb{R}}$-type) if $g$ is a $D$-Green function of $C^{\infty}$-type (resp. of $C^{0}$-type, of $\mathrm{PSH}_{\mathbb{R}}$-type). More generally, for a fixed type $\mathscr{T}$ for Green functions, $\bar{D}$ is said to be of $\mathscr{T}$-type if $g$ is a $D$-Green function of $\mathscr{T}$-type. For arithmetic $\mathbb{R}$-Cartier divisors $\bar{D}_{1}=\left(D_{1}, g_{1}\right)$ and $\bar{D}_{2}=\left(D_{2}, g_{2}\right)$, we define

$$
\begin{aligned}
& \bar{D}_{1}=\bar{D}_{2} \stackrel{\text { def }}{\Longleftrightarrow} D_{1}=D_{2} \text { and } g_{1}=g_{2} \text { (a.e.), } \\
& \bar{D}_{1} \leq \bar{D}_{2} \stackrel{\text { def }}{\Longleftrightarrow} D_{1} \leq D_{2} \text { and } g_{1} \leq g_{2} \text { (a.e.). }
\end{aligned}
$$

If $\bar{D} \geq(0,0)$, then $\bar{D}$ is said to be effective. Further, the set

$\{\bar{M} \mid \bar{M}$ is an arithmetic $\mathbb{R}$-Cartier divisor on $X$ and $\bar{M} \leq \bar{D}\}$

is denoted by $(-\infty, \bar{D}]$.

- Volume of arithmetic $\mathbb{R}$-Cartier divisors of $C^{0}$-type. Let $\widehat{\operatorname{Div}}_{C^{0}}(X)_{\mathbb{R}}$ be the group of arithmetic $\mathbb{R}$-Cartier divisors of $C^{0}$-type on $X$. For $\bar{D} \in \widehat{\operatorname{Div}}_{C^{0}}(X)_{\mathbb{R}}$, we define

$$
\begin{aligned}
& H^{0}(X, D):=\left\{\begin{array}{l|l}
\psi & \begin{array}{l}
\psi \text { is a non-zero rational function } \\
\text { on } X \text { with }(\psi)+D \geq 0
\end{array}
\end{array}\right\} \cup\{0\}, \\
& \hat{H}^{0}(X, \bar{D}):=\left\{\psi \in H^{0}(X, D) \mid\|\psi\|_{g} \leq 1\right\}, \\
& \hat{h}^{0}(X, \bar{D}):=\log \#\left(\hat{H}^{0}(X, \bar{D})\right) \text {, } \\
& \widehat{\operatorname{vol}}(\bar{D}):=\limsup _{n \rightarrow \infty} \frac{\hat{h}^{0}(X, n \bar{D})}{n^{d} / d !} .
\end{aligned}
$$

Note that

$$
\hat{H}^{0}(X, \bar{D})=\left\{\begin{array}{l|l}
\psi & \begin{array}{l}
\psi \text { is a non-zero rational function } \\
\text { on } X \text { with } \widehat{(\psi)}+\bar{D} \geq(0,0)
\end{array}
\end{array}\right\} \cup\{0\} .
$$


The continuity of

$$
\widehat{\operatorname{vol}}: \widehat{\operatorname{Pic}}(X)_{\mathbb{Q}} \rightarrow \mathbb{R}
$$

is proved in [14], where $\widehat{\operatorname{Pic}}(X)_{\mathbb{Q}}:=\widehat{\operatorname{Pic}}(X) \otimes_{\mathbb{Z}} \mathbb{Q}$. Moreover, in [15], we introduce $\widehat{\operatorname{Pic}}_{C^{0}}(X)_{\mathbb{R}}$ as a natural extension of $\widehat{\operatorname{Pic}}(X)_{\mathbb{Q}}($ for details, see [15] or Subsection 5.1) and prove that $\widehat{\operatorname{vol}}: \widehat{\mathrm{Pic}}(X)_{\mathbb{Q}} \rightarrow \mathbb{R}$ has a continuous extension

$$
\widehat{\operatorname{vol}}: \widehat{\operatorname{Pic}}_{C^{0}}(X)_{\mathbb{R}} \rightarrow \mathbb{R} \text {. }
$$

Theorem 5.2.2 shows that there is a natural surjective homomorphism

$$
\overline{\mathscr{O}}_{\mathbb{R}}: \widehat{\operatorname{Div}}_{C^{0}}(X)_{\mathbb{R}} \rightarrow \widehat{\operatorname{Pic}}_{C^{0}}(X)_{\mathbb{R}}
$$

such that $\widehat{\operatorname{vol}}(\bar{D})=\widehat{\operatorname{vol}}\left(\overline{\mathscr{O}}_{\mathbb{R}}(\bar{D})\right)$ for all $\bar{D} \in \widehat{\operatorname{Div}}_{C^{0}}(X)_{\mathbb{R}}$. In particular, by using results in [5], [6], [14], [15], [16] and [23], we have the following properties of $\widehat{\mathrm{vol}}: \widehat{\operatorname{Div}}_{C^{0}}(X)_{\mathbb{R}} \rightarrow \mathbb{R}(\mathrm{cf}$. Theorems 5.2.2 and 6.6.1):

(1) $\widehat{\operatorname{vol}}: \widehat{\operatorname{Div}}_{C^{0}}(X)_{\mathbb{R}} \rightarrow \mathbb{R}$ is positively homogeneous of degree $d$, that is, $\widehat{\operatorname{vol}}(a \bar{D})=$ $a^{d} \widehat{\operatorname{vol}}(\bar{D})$ for all $a \in \mathbb{R}_{\geq 0}$ and $\bar{D} \in \widehat{\operatorname{Div}}_{C^{0}}(X)_{\mathbb{R}}(\mathrm{cf}$. [14], [15]).

(2) $\widehat{\text { vol }}: \widehat{\operatorname{Div}}_{C^{0}}(X)_{\mathbb{R}} \rightarrow \mathbb{R}$ is continuous in the following sense: Let $\bar{D}_{1}, \ldots, \bar{D}_{r}$, $\bar{A}_{1}, \ldots, \bar{A}_{r^{\prime}}$ be arithmetic $\mathbb{R}$-Cartier divisors of $C^{0}$-type. For a compact set $B$ in $\mathbb{R}^{r}$ and a positive number $\epsilon$, there are positive numbers $\delta$ and $\delta^{\prime}$ such that, for all $a_{1}, \ldots, a_{r}, \delta_{1}, \ldots, \delta_{r^{\prime}} \in \mathbb{R}$ and $\phi \in C^{0}(X)$ with $\left(a_{1}, \ldots, a_{r}\right) \in B$, $\sum_{j=1}^{r^{\prime}}\left|\delta_{j}\right| \leq \delta$ and $\|\phi\|_{\text {sup }} \leq \delta^{\prime}$, we have

$$
\left|\widehat{\operatorname{vol}}\left(\sum_{i=1}^{r} a_{i} \bar{D}_{i}+\sum_{j=1}^{r^{\prime}} \delta_{j} \bar{A}_{j}+(0, \phi)\right)-\widehat{\operatorname{vol}}\left(\sum_{i=1}^{r} a_{i} \bar{D}_{i}\right)\right| \leq \epsilon .
$$

Moreover, if $\bar{D}_{1}, \ldots, \bar{D}_{r}, \bar{A}_{1}, \ldots, \bar{A}_{r^{\prime}}$ are $C^{\infty}$, then there is a positive constant $C$ depending only on $X$ and $\bar{D}_{1}, \ldots, \bar{D}_{r}, \bar{A}_{1}, \ldots, \bar{A}_{r^{\prime}}$ such that

$$
\begin{array}{r}
\left|\widehat{\operatorname{vol}}\left(\sum_{i=1}^{r} a_{i} \bar{D}_{i}+\sum_{j=1}^{r^{\prime}} \delta_{j} \bar{A}_{j}+(0, \phi)\right)-\widehat{\operatorname{vol}}\left(\sum_{i=1}^{r} a_{i} \bar{D}_{i}\right)\right| \\
\leq C\left(\sum_{i=1}^{r}\left|a_{i}\right|+\sum_{j=1}^{r^{\prime}}\left|\delta_{j}\right|\right)^{d-1}\left(\|\phi\|_{\text {sup }}+\sum_{j=1}^{r^{\prime}}\left|\delta_{j}\right|\right)
\end{array}
$$

for all $a_{1}, \ldots, a_{r}, \delta_{1}, \ldots, \delta_{r^{\prime}} \in \mathbb{R}$ and $\phi \in C^{0}(X)$ (cf. [14], [15]).

(3) $\widehat{\operatorname{vol}}(\bar{D})$ is given by "lim", that is,

$$
\widehat{\operatorname{vol}}(\bar{D})=\lim _{t \rightarrow \infty} \frac{\hat{h}^{0}(t \bar{D})}{t^{d} / d !}
$$

where $\bar{D} \in \widehat{\operatorname{Div}}_{C^{0}}(X)_{\mathbb{R}}$ and $t \in \mathbb{R}_{>0}$ (cf. [5], [15]). 
(4) $\widehat{\operatorname{vol}}(-)^{1 / d}$ is concave, that is, for arithmetic $\mathbb{R}$-Cartier divisors $\bar{D}_{1}, \bar{D}_{2}$ of $C^{0}$ type, if $\bar{D}_{1}$ and $\bar{D}_{2}$ are pseudo-effective (for the definition of pseudo-effectivity, see Subsection 6.1), then

$$
\widehat{\operatorname{vol}}\left(\bar{D}_{1}+\bar{D}_{2}\right)^{1 / d} \geq \widehat{\operatorname{vol}}\left(\bar{D}_{1}\right)^{1 / d}+\widehat{\operatorname{vol}}\left(\bar{D}_{2}\right)^{1 / d}
$$

(cf. [16], [23]).

(5) (Fujita's approximation theorem for $\mathbb{R}$-Cartier divisors) If $\bar{D}$ is an arithmetic $\mathbb{R}$-Cartier divisor of $C^{0}$-type and $\widehat{\operatorname{vol}}(\bar{D})>0$, then, for any positive number $\epsilon$, there are a birational morphism $\mu: Y \rightarrow X$ of generically smooth and normal projective arithmetic varieties and an ample arithmetic $\mathbb{Q}$-Cartier divisor $\bar{A}$ of $C^{\infty}$-type on $Y$ (cf. Section 6) such that $\bar{A} \leq \mu^{*}(\bar{D})$ and $\widehat{\operatorname{vol}}(\bar{A}) \geq \widehat{\operatorname{vol}}(\bar{D})-\epsilon$ (cf. [6], [23]).

(6) (The generalized Hodge index theorem for $\mathbb{R}$-Cartier divisors) If $\bar{D}$ is an arithmetic $\mathbb{R}$-Cartier divisor of $\left(C^{0} \cap \mathrm{PSH}\right)$-type and $D$ is nef on every fiber of $X \rightarrow \operatorname{Spec}(\mathbb{Z})$, then $\widehat{\operatorname{vol}}(\bar{D}) \geq \widehat{\operatorname{deg}}\left(\bar{D}^{d}\right)$ (see descriptions in "Positivity of arithmetic $\mathbb{R}$-Cartier divisors" below or Proposition 6.4.2 for the definition of $\left.\widehat{\operatorname{deg}}\left(\bar{D}^{d}\right)\right)$ (cf. [14]).

- Intersection number of an arithmetic $\mathbb{R}$-Cartier divisor with a 1-dimensional subscheme. Let $\mathscr{T}$ be a real valued type for Green functions such that $C^{0} \subseteq \mathscr{T}$ and $-u \in \mathscr{T}$ whenever $u \in \mathscr{T}$. Let $\bar{D}=(D, g)$ be an arithmetic $\mathbb{R}$-Cartier divisor of $\mathscr{T}$-type. Let $C$ be a 1-dimensional closed integral subscheme of $X$. Let $D=a_{1} D_{1}+\cdots+a_{l} D_{l}$ be a decomposition such that $a_{1}, \ldots, a_{l} \in \mathbb{R}$ and $D_{i}$ 's are Cartier divisors. For simplicity, we assume that $D_{i}$ 's are effective, $C \nsubseteq \operatorname{Supp}\left(D_{i}\right)$ for all $i$ and that $C$ is flat over $\mathbb{Z}$. In this case, we define

$$
\widehat{\operatorname{deg}}\left(\left.\bar{D}\right|_{C}\right):=\sum_{i=1}^{l} a_{i} \log \#\left(\mathscr{O}_{C}\left(D_{i}\right) / \mathscr{O}_{C}\right)+\frac{1}{2} \sum_{x \in C(\mathbb{C})} g_{\mathrm{can}}(x)
$$

For the general case, see Section 5.3. Let $Z$ be a 1-cycle on $X$ with coefficients in $\mathbb{R}$, that is, there are $a_{1}, \ldots, a_{l} \in \mathbb{R}$ and 1-dimensional closed integral subschemes $C_{1}, \ldots, C_{l}$ on $X$ such that $Z=a_{1} C_{1}+\cdots+a_{l} C_{l}$. Then we define

$$
\widehat{\operatorname{deg}}(\bar{D} \mid Z):=\sum_{i=1}^{l} a_{i} \widehat{\operatorname{deg}}\left(\left.\bar{D}\right|_{C_{i}}\right) .
$$

- Positivity of arithmetic $\mathbb{R}$-Cartier divisors. An arithmetic $\mathbb{R}$-Cartier divisor $\bar{D}$ is said to be nef if $\bar{D}$ is of $\mathrm{PSH}_{\mathbb{R}}$-type and $\widehat{\operatorname{deg}}\left(\left.D\right|_{C}\right) \geq 0$ for all 1-dimensional closed integral subschemes $C$ of $X$. The cone of all nef arithmetic $\mathbb{R}$-Cartier divisors on $X$ is denoted by $\widehat{\operatorname{Nef}}(X)_{\mathbb{R}}$. Moreover, the cone of all nef arithmetic 
$\mathbb{R}$-Cartier divisors of $C^{\infty}$-type (resp. $C^{0}$-type) on $X$ is denoted by $\widehat{\operatorname{Nef}}_{C^{\infty}}(X)_{\mathbb{R}}$ (resp. $\left.\widehat{\operatorname{Nef}}_{C^{0}}(X)_{\mathbb{R}}\right)$. Further, we say $\bar{D}$ is big if $\widehat{\operatorname{vol}}(\bar{D})>0$.

Let $\widehat{\operatorname{Div}}_{C^{0}}$ Nef $(X)_{\mathbb{R}}$ be the vector subspace of $\widehat{\operatorname{Div}}_{C^{0}}(X)_{\mathbb{R}}$ generated by $\widehat{\operatorname{Nef}}_{C^{0}}(X)_{\mathbb{R}}$. Then, by Proposition 6.4.2,

$$
\widehat{\operatorname{Div}}_{C^{\infty}}(X)_{\mathbb{R}}+\widehat{\operatorname{Div}}_{C^{0} \cap \mathrm{PSH}}(X)_{\mathbb{R}} \subseteq \widehat{\operatorname{Div}}_{C^{0}}^{\mathrm{Nef}}(X)_{\mathbb{R}}
$$

and the symmetric multi-linear map

$$
\widehat{\operatorname{Div}}_{C \infty}(X)_{\mathbb{R}} \times \cdots \times \widehat{\operatorname{Div}}_{C \infty}(X)_{\mathbb{R}} \rightarrow \mathbb{R}
$$

given by $\left(\bar{D}_{1}, \ldots, \bar{D}_{d}\right) \mapsto \widehat{\operatorname{deg}}\left(\bar{D}_{1} \cdots \bar{D}_{d}\right)$ (cf. Proposition-Definition 6.4.1) extends to a unique symmetric multi-linear map

$$
\widehat{\operatorname{Div}}_{C^{0}}^{\mathrm{Nef}}(X)_{\mathbb{R}} \times \cdots \times \widehat{\operatorname{Div}}_{C^{0}}^{\mathrm{Nef}}(X)_{\mathbb{R}} \rightarrow \mathbb{R}
$$

such that $(\bar{D}, \ldots, \bar{D}) \mapsto \widehat{\operatorname{vol}}(\bar{D})$ for $\bar{D} \in \widehat{\operatorname{Nef}}_{C^{0}}(X)_{\mathbb{R}}$.

\section{§0.3. Zariski decompositions on arithmetic surfaces}

Let $X$ be a regular projective arithmetic surface. The main theorem of this paper is the following:

Theorem A (cf. Theorems 9.2.1 and 9.3.5). Let $\bar{D}$ be an arithmetic $\mathbb{R}$-Cartier divisor of $C^{0}$-type on $X$ such that the set

$$
\begin{aligned}
& (-\infty, \bar{D}] \cap \widehat{\operatorname{Nef}}(X)_{\mathbb{R}} \\
& \quad=\{\bar{M} \mid \bar{M} \text { is a nef arithmetic } \mathbb{R} \text {-Cartier divisor on } X \text { and } \bar{M} \leq \bar{D}\}
\end{aligned}
$$

is not empty. Then there is a nef arithmetic $\mathbb{R}$-Cartier divisor $\bar{P}$ of $C^{0}$-type which is the greatest element of $(-\infty, \bar{D}] \cap \widehat{\operatorname{Nef}}(X)_{\mathbb{R}}$, that is, $\bar{P} \in(-\infty, \bar{D}] \cap \widehat{\operatorname{Nef}}(X)_{\mathbb{R}}$ and $\bar{M} \leq \bar{P}$ for all $\bar{M} \in(-\infty, \bar{D}] \cap \widehat{\operatorname{Nef}}(X)_{\mathbb{R}}$. Moreover, if we set $\bar{N}=\bar{D}-\bar{P}$, then the following properties hold:

(1) $\widehat{\operatorname{vol}}(\bar{D})=\widehat{\operatorname{vol}}(\bar{P})=\widehat{\operatorname{deg}}\left(\bar{P}^{2}\right)$.

(2) $\widehat{\operatorname{deg}}\left(\left.\bar{P}\right|_{C}\right)=0$ for all 1-dimensional closed integral subschemes $C$ with $C \subseteq$ $\operatorname{Supp}(N)$.

(3) If $\bar{L}$ is an arithmetic $\mathbb{R}$-Cartier divisor of $\mathrm{PSH}_{\mathbb{R}}$-type on $X$ such that $0 \leq \bar{L} \leq$ $\bar{N}$ and $\operatorname{deg}\left(\left.\bar{L}\right|_{C}\right) \geq 0$ for all 1-dimensional closed integral subschemes $C$ with $C \subseteq \operatorname{Supp}(N)$, then $\bar{L}=0$.

Note that the condition $(-\infty, \bar{D}] \cap \widehat{\operatorname{Nef}}(X)_{\mathbb{R}} \neq \emptyset$ is guaranteed if $\hat{h}^{0}(X, a \bar{D}) \neq 0$ for some $a \in \mathbb{R}_{>0}$ (cf. Proposition 9.3.2). The above decomposition $\bar{D}=\bar{P}+\bar{N}$ is 
called the Zariski decomposition of $\bar{D}$ and we say $\bar{P}$ (resp. $\bar{N}$ ) is the positive part (resp. the negative part) of the decomposition. For example, let $\mathbb{P}_{\mathbb{Z}}^{1}=\operatorname{Proj}(\mathbb{Z}[x, y])$, $C_{0}=\{x=0\}, z=x / y$ and $\alpha, \beta \in \mathbb{R}_{>0}$ with $\alpha>1$ and $\beta<1$. Then the positive part of the arithmetic Cartier divisor

$$
\left(C_{0},-\log |z|^{2}+\log \max \left\{\alpha^{2}|z|^{2}, \beta^{2}\right\}\right)
$$

of $\left(C^{0} \cap \mathrm{PSH}\right)$-type on $\mathbb{P}_{\mathbb{Z}}^{1}$ is

$$
\left(\theta C_{0},-\theta \log |z|^{2}+\log \max \left\{\alpha^{2}|z|^{2 \theta}, 1\right\}\right),
$$

where $\theta=\log \alpha /(\log \alpha-\log \beta)$ (cf. Subsection 9.4). This example shows that an $\mathbb{R}$-Cartier divisor is necessary for the arithmetic Zariski decomposition. In addition, an example in Remark 9.4.3 shows that the Arakelov Chow group consisting of admissible metrics due to Arakelov-Faltings is insufficient to get the Zariski decomposition.

Assume that $N \neq 0$. Let $N=c_{1} C_{1}+\cdots+c_{l} C_{l}$ be the decomposition of $N$ such that $c_{1}, \ldots, c_{l} \in \mathbb{R}_{>0}$ and $C_{i}$ 's are distinct 1-dimensional closed integral subschemes on $X$. Let $\left(C_{1}, g_{1}\right), \ldots,\left(C_{l}, g_{l}\right)$ be effective arithmetic Cartier divisors of $\mathrm{PSH}_{\mathbb{R}^{-}}$ type such that

$$
c_{1}\left(C_{1}, g_{1}\right)+\cdots+c_{l}\left(C_{l}, g_{l}\right) \leq \bar{N},
$$

which is possible by Proposition 2.4.2 and Lemma 9.1.3. Then, by Lemma 1.2.3, item (3) above yields the inequality

$$
(-1)^{l} \operatorname{det}\left(\widehat{\operatorname{deg}}\left(\left.\left(C_{i}, g_{i}\right)\right|_{C_{j}}\right)\right)>0 .
$$

This is a counterpart of the property (4.2) of the Zariski decomposition on an algebraic surface. On the other hand, our Zariski decomposition is a refinement of Fujita's approximation theorem due to Chen [6] and Yuan [23] on an arithmetic surface. Actually Fujita's approximation theorem on an arithmetic surface is a consequence of the above theorem (cf. Proposition 9.3.7).

Let $\bar{D}$ be an effective arithmetic $\mathbb{R}$-Cartier divisor of $C^{0}$-type. For each $n \geq 1$, we define

$$
\begin{aligned}
F_{n}(\bar{D}) & =\frac{1}{n} \sum_{C} \min \left\{\operatorname{mult}_{C}((\phi)+n D) \mid \phi \in \hat{H}^{0}(X, n \bar{D}) \backslash\{0\}\right\} C, \\
M_{n}(\bar{D}) & =D-F_{n}(\bar{D}) .
\end{aligned}
$$

Let $V(n \bar{D})$ be the complex vector space generated by $\hat{H}^{0}(X, n \bar{D})$. It is easy to see that

$$
g_{M_{n}(\bar{D})}:=g+\frac{1}{n} \log \operatorname{dist}(V(n \bar{D}) ; n g)
$$


is an $M_{n}(\bar{D})$-Green function of $C^{\infty}$-type (for the definition of distorsion functions, see Subsection 3.2). Then we have the following:

Theorem B (Asymptotic orthogonality). If $\bar{D}$ is big, then

$$
\lim _{n \rightarrow \infty} \widehat{\operatorname{deg}}\left(\left(M_{n}(\bar{D}), g_{M_{n}(\bar{D})}\right) \mid F_{n}(\bar{D})\right)=0 .
$$

\section{§0.4. Technical results for the proof of the arithmetic Zariski decomposition}

In order to get the greatest element of $(-\infty, \bar{D}] \cap \widehat{\operatorname{Nef}}(X)_{\mathbb{R}}$, we need to consider the nefness of the limit of a convergent sequence of nef arithmetic $\mathbb{R}$-Cartier divisors. The following theorem is our solution for this problem:

Theorem $\mathbf{C}$ (cf. Theorem 7.1). Let $X$ be a regular projective arithmetic surface. Let $\left\{\bar{M}_{n}=\left(M_{n}, h_{n}\right)\right\}_{n=0}^{\infty}$ be a sequence of nef arithmetic $\mathbb{R}$-Cartier divisors on $X$ with the following properties:

(a) There is an arithmetic Cartier divisor $\bar{D}=(D, g)$ of $C^{0}$-type such that $\bar{M}_{n} \leq$ $\bar{D}$ for all $n \geq 1$.

(b) There is a proper closed subset $E$ of $X$ such that $\operatorname{Supp}(D) \subseteq E$ and $\operatorname{Supp}\left(M_{n}\right)$ $\subseteq E$ for all $n \geq 1$.

(c) $\lim _{n \rightarrow \infty} \operatorname{mult}_{C}\left(M_{n}\right)$ exists for all 1-dimensional closed integral subschemes $C$ on $X$.

(d) $\lim \sup _{n \rightarrow \infty}\left(h_{n}\right)_{\text {can }}(x)$ exists in $\mathbb{R}$ for all $x \in X(\mathbb{C}) \backslash E(\mathbb{C})$.

Then there is a nef arithmetic $\mathbb{R}$-Cartier divisor $\bar{M}=(M, h)$ on $X$ such that $\bar{M} \leq \bar{D}$

$$
M=\sum_{C}\left(\lim _{n \rightarrow \infty} \operatorname{mult}_{C}\left(M_{n}\right)\right) C
$$

and $\left.h_{\mathrm{can}}\right|_{X(\mathbb{C}) \backslash E(\mathbb{C})}$ is the upper semicontinuous regularization of the function given by $x \mapsto \lim \sup _{n \rightarrow \infty}\left(h_{n}\right)_{\text {can }}(x)$ over $X(\mathbb{C}) \backslash E(\mathbb{C})$.

Moreover, for the first property $\widehat{\operatorname{vol}}(\bar{P})=\widehat{\operatorname{vol}}(\bar{D})$ of the arithmetic Zariski decomposition, it is necessary to observe the following behavior of distorsion functions (cf. Remark 9.3.9), which is a consequence of Gromov's inequality for an $\mathbb{R}$-Cartier divisor (cf. Proposition 3.1.1).

Theorem D (cf. Theorem 3.2.3). Let $V$ be an equidimensional smooth projective variety over $\mathbb{C}$ and let $D$ be an $\mathbb{R}$-Cartier divisor on $V$. Let $R=\bigoplus_{n \geq 0} R_{n}$ be a graded subring of $\bigoplus_{n \geq 0} H^{0}(V, n D)$. If $g$ is a $D$-Green function of $C^{\infty}$-type, then there is a positive constant $C$ with the following properties: 
(1) $\operatorname{dist}\left(R_{n} ; n g\right) \leq C(n+1)^{3 \operatorname{dim} V}$ for all $n \geq 0$.

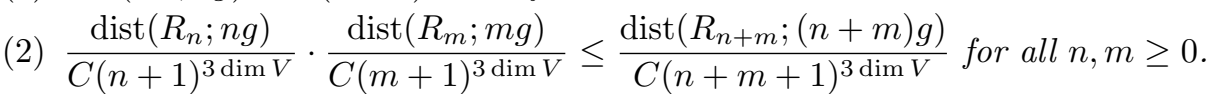

The most difficult point for the proof of the arithmetic Zariski decomposition is to check the continuity of the positive part. For this purpose, the following theorem is needed:

Theorem E (cf. Theorem 4.6). Let $V$ be an equidimensional smooth projective variety over $\mathbb{C}$. Let $A$ and $B$ be $\mathbb{R}$-Cartier divisors on $V$ with $A \leq B$. If there is an $A$-Green function $h$ of $C^{\infty}$-type such that $d d^{c}([h])+\delta_{A}$ is represented by either a positive $C^{\infty}$-form or the zero form, then, for a B-Green function $g_{B}$ of $C^{0}$-type, there is an A-Green function $g$ of $\left(C^{0} \cap \mathrm{PSH}\right)$-type such that $g$ is the greatest element of the set

$$
\left.G_{\mathrm{PSH}}(V ; A)_{\leq g_{B}}:=\left\{u \in G_{\mathrm{PSH}}(V ; A) \mid u \leq g_{B} \text { (a.e. }\right)\right\}
$$

modulo null functions, that is, $g \in G_{\mathrm{PSH}}(V ; A)_{\leq g_{B}}$ and $u \leq g$ (a.e.) for all $u \in$ $G_{\mathrm{PSH}}(V ; A)_{\leq g_{B}}$.

For the proof, we actually use a recent regularity result due to BermanDemailly [3]. Even starting from an arithmetic Cartier divisor $\bar{D}$ of $C^{\infty}$-type, it is not expected that the positive part $\bar{P}$ is of $C^{\infty}$-type again (cf. [17]). It could be that $\bar{P}$ is of $C^{1,1}$-type.

\section{§1. Zariski decompositions in vector spaces}

Logically the material of this section is not necessary except Lemma 1.2.3. However, it gives an elementary case for our considerations and provides a good overview of our paper.

\section{$\S 1.1$}

In [1], Bauer presents a simple proof of the existence of Zariski decompositions on an algebraic surface. Unfortunately, he uses linear series on the algebraic surface to show the negative definiteness of the negative part of the Zariski decomposition. In this section, we give a linear algebraic proof without using any material of algebraic geometry. The main technical result for our purpose is Lemma 1.2.3. After the first draft of this paper was completed, Bauer, Caibăr and Kennedy kindly informed me that, in [2], they independently obtained several results similar to those of this section. Their paper is written for a general reader. 
Let $V$ be a vector space over $\mathbb{R}$. Let $\boldsymbol{e}=\left\{e_{\lambda}\right\}_{\lambda \in \Lambda}$ be a basis of $V$ and let $\phi=\left\{\phi_{\lambda}\right\}_{\lambda \in \Lambda}$ be a family of elements of $\operatorname{Hom}_{\mathbb{R}}(V, \mathbb{R})$ such that $\phi_{\lambda}\left(e_{\mu}\right) \geq 0$ for $\lambda \neq \mu$. The pair $(\boldsymbol{e}, \boldsymbol{\phi})$ is called a system of Zariski decompositions in $V$.

Let us fix several notations which are used only in this section. For $\lambda \in \Lambda$, the coefficient of $e_{\lambda}$ in the expansion of $x$ with respect to the basis $\boldsymbol{e}$ is denoted by $x(\lambda ; \boldsymbol{e})$, that is, $x=\sum_{\lambda} x(\lambda ; \boldsymbol{e}) e_{\lambda}$. Let $\leq_{\boldsymbol{e}}$ be order relation on $V$ given by

$$
x \leq_{\boldsymbol{e}} y \stackrel{\text { def }}{\Longleftrightarrow} x(\lambda ; \boldsymbol{e}) \leq y(\lambda ; \boldsymbol{e}) \text { for all } \lambda \in \Lambda \text {. }
$$

We often use $y \geq_{\boldsymbol{e}} x$ instead of $x \leq_{\boldsymbol{e}} y \cdot \operatorname{Supp}(x ; \boldsymbol{e}),[x, y]_{\boldsymbol{e}},(-\infty, x]_{\boldsymbol{e}},[x, \infty)_{\boldsymbol{e}}$, $\operatorname{Nef}(\boldsymbol{\phi})$ and $\operatorname{Num}(\boldsymbol{\phi})$ are defined as follows:

$$
\begin{aligned}
\operatorname{Supp}(x ; \boldsymbol{e}) & :=\{\lambda \in \Lambda \mid x(\lambda ; \boldsymbol{e}) \neq 0\}, \\
{[x, y]_{\boldsymbol{e}} } & :=\left\{v \in V \mid x \leq_{\boldsymbol{e}} v \leq_{\boldsymbol{e}} y\right\}, \\
(-\infty, x]_{\boldsymbol{e}} & :=\left\{v \in V \mid v \leq_{\boldsymbol{e}} x\right\}, \\
{[x, \infty)_{\boldsymbol{e}} } & :=\left\{v \in V \mid v \geq_{\boldsymbol{e}} x\right\}, \\
\operatorname{Nef}(\boldsymbol{\phi}) & :=\left\{v \in V \mid \phi_{\lambda}(v) \geq 0 \text { for all } \lambda \in \Lambda\right\}, \\
\operatorname{Num}(\boldsymbol{\phi}) & :=\left\{v \in V \mid \phi_{\lambda}(v)=0 \text { for all } \lambda \in \Lambda\right\} .
\end{aligned}
$$

For an element $x$ of $V$, a decomposition $x=y+z$ is called a Zariski decomposition of $x$ with respect to $(\boldsymbol{e}, \boldsymbol{\phi})$ if the following conditions are satisfied:

(1) $y \in \operatorname{Nef}(\phi)$ and $z \geq_{\boldsymbol{e}} 0$.

(2) $\phi_{\lambda}(y)=0$ for all $\lambda \in \operatorname{Supp}(z ; \boldsymbol{e})$.

(3) $\left\{x \in \sum_{\lambda \in \operatorname{Supp}(z ; \boldsymbol{e})} \mathbb{R}_{\geq 0} e_{\lambda} \mid \phi_{\lambda}(x) \geq 0\right.$ for all $\left.\lambda \in \operatorname{Supp}(z ; \boldsymbol{e})\right\}=\{0\}$.

We call $y$ (resp. $z$ ) the positive part of $x$ (resp. negative part of $x$ ).

The purpose of this section is to give the proof of the following proposition.

Proposition 1.1.1. For an element $x$ of $V$, we have the following:

(1) The following are equivalent:

(1.1) A Zariski decomposition of $x$ with respect to $(\boldsymbol{e}, \boldsymbol{\phi})$ exists.

$(1.2)(-\infty, x]_{\boldsymbol{e}} \cap \operatorname{Nef}(\phi) \neq \emptyset$.

(2) If a Zariski decomposition exists, then it is uniquely determined.

(3) If a Zariski decomposition of $x$ with respect to $(\boldsymbol{e}, \boldsymbol{\phi})$ exists and the negative part $z$ of $x$ is non-zero, then $z$ has the following properties:

(3.1) Let $Q$ be the matrix $\left(\phi_{\lambda}\left(e_{\mu}\right)\right)_{\lambda, \mu \in \operatorname{Supp}(z ; \boldsymbol{e})}$. Then

$$
(-1)^{\#(\operatorname{Supp}(z ; \boldsymbol{e}))} \operatorname{det} Q>0 \text {. }
$$

Moreover, if $Q$ is symmetric, then $Q$ is negative definite.

(3.2) $\left\{e_{\lambda}\right\}_{\lambda \in \operatorname{Supp}(z ; \boldsymbol{e})}$ is linearly independent on $V / \operatorname{Num}(\boldsymbol{\phi})$. 


\section{$\S 1.2$. Proofs}

Here we prove Proposition 1.1.1.

For $x_{1}, \ldots, x_{r} \in V, \max _{\boldsymbol{e}}\left\{x_{1}, \ldots, x_{r}\right\} \in V$ is given by

$$
\max _{\boldsymbol{e}}\left\{x_{1}, \ldots, x_{r}\right\}:=\sum_{\lambda \in \Lambda} \max \left\{x_{1}(\lambda ; \boldsymbol{e}), \ldots, x_{r}(\lambda ; \boldsymbol{e})\right\} e_{\lambda} .
$$

Let us begin with the following lemma.

Lemma 1.2.1. If $x_{1}, \ldots, x_{r} \in \operatorname{Nef}(\boldsymbol{\phi})$, then $\max \left\{x_{1}, \ldots, x_{r}\right\} \in \operatorname{Nef}(\boldsymbol{\phi})$.

Proof. It is sufficient to see that if $\phi_{\lambda}\left(x_{i}\right) \geq 0$ for all $i$, then $\phi_{\lambda}\left(\max _{\boldsymbol{e}}\left\{x_{1}, \ldots, x_{r}\right\}\right)$ $\geq 0$. We set $z=\max _{\boldsymbol{e}}\left\{x_{1}, \ldots, x_{r}\right\}$. Note that $\operatorname{Supp}\left(z-x_{1} ; \boldsymbol{e}\right) \cap \cdots \cap \operatorname{Supp}\left(z-x_{r} ; \boldsymbol{e}\right)$ $=\emptyset$. Thus there is $i$ with $\lambda \notin \operatorname{Supp}\left(z-x_{i} ; \boldsymbol{e}\right)$. Then $\phi_{\lambda}\left(z-x_{i}\right) \geq 0$, and hence

$$
\phi_{\lambda}(z)=\phi_{\lambda}\left(z-x_{i}\right)+\phi_{\lambda}\left(x_{i}\right) \geq 0 .
$$

Lemma 1.2.2. Let $x$ be an element of $V$ such that $(-\infty, x]_{\boldsymbol{e}} \cap \operatorname{Nef}(\boldsymbol{\phi}) \neq \emptyset$. Then there is the greatest element $y$ in $(-\infty, x]_{\boldsymbol{e}} \cap \operatorname{Nef}(\boldsymbol{\phi})$, that is, $y \in \operatorname{Nef}(\boldsymbol{\phi}) \cap(-\infty, x]_{\boldsymbol{e}}$ and $y \geq_{\boldsymbol{e}} v$ for all $v \in \operatorname{Nef}(\phi) \cap(-\infty, x]_{\boldsymbol{e}}$. This greatest element $y$ is denoted by

$$
\max \left(\operatorname{Nef}(\phi) \cap(-\infty, x]_{\boldsymbol{e}}\right) .
$$

Further, $y$ and $z:=x-y$ have the following properties:

(a) $y \in \operatorname{Nef}(\phi), z \geq_{\boldsymbol{e}} 0$ and $x=y+z$.

(b) $\phi_{\lambda}(y)=0$ for all $\lambda \in \operatorname{Supp}(z ; \boldsymbol{e})$.

(c) $\left\{v \in \sum_{\lambda \in \operatorname{Supp}(z ; \boldsymbol{e})} \mathbb{R}_{\geq 0} e_{\lambda} \mid \phi_{\lambda}(v) \geq 0\right.$ for all $\left.\lambda \in \operatorname{Supp}(z ; \boldsymbol{e})\right\}=\{0\}$.

Proof. We choose $x^{\prime} \in(-\infty, x]_{\boldsymbol{e}} \cap \operatorname{Nef}(\boldsymbol{\phi})$. Let us prove the following claim.

Claim 1.2.2.1. There is a greatest element $y$ of $\operatorname{Nef}(\phi) \cap\left[x^{\prime}, x\right]_{e}$.

Proof. Note that $\left[x^{\prime}, x\right]_{\boldsymbol{e}}=x^{\prime}+\left[0, x-x^{\prime}\right]_{\boldsymbol{e}}$. Moreover, it is easy to see that

$$
\begin{aligned}
\operatorname{Nef}(\boldsymbol{\phi}) \cap\left[x^{\prime}, x\right]_{\boldsymbol{e}} & \\
& =x^{\prime}+\left\{v \in\left[0, x-x^{\prime}\right]_{\boldsymbol{e}} \mid \phi_{\lambda}(v) \geq-\phi_{\lambda}\left(x^{\prime}\right) \text { for all } \lambda \in \operatorname{Supp}\left(x-x^{\prime} ; \boldsymbol{e}\right)\right\} .
\end{aligned}
$$

Therefore, $\operatorname{Nef}(\phi) \cap\left[x^{\prime}, x\right]_{\boldsymbol{e}}$ is a translation of a bounded convex polyhedral set in a finite-dimensional vector space $\bigoplus_{\lambda \in \operatorname{Supp}\left(x-x^{\prime} ; \boldsymbol{e}\right)} \mathbb{R} e_{\lambda}$. Hence $\operatorname{Nef}(\boldsymbol{\phi}) \cap\left[x^{\prime}, x\right]_{\boldsymbol{e}}$ is a convex polytope, that is, there are $\gamma_{1}, \ldots, \gamma_{l} \in \operatorname{Nef}(\boldsymbol{\phi}) \cap\left[x^{\prime}, x\right]_{\boldsymbol{e}}$ such that $\operatorname{Nef}(\boldsymbol{\phi}) \cap$ $\left[x^{\prime}, x\right]_{\boldsymbol{e}}=\operatorname{Conv}\left\{\gamma_{1}, \ldots, \gamma_{l}\right\}$ (cf. [22, Theorem 3.2.5 or Finite basis theorem]). If we set $y=\max \left\{\gamma_{1}, \ldots, \gamma_{l}\right\}$, then, by Lemma 1.2.1, $y \in \operatorname{Nef}(\boldsymbol{\phi}) \cap\left[x^{\prime}, x\right]_{\boldsymbol{e}}$. Moreover, 
for $v=a_{1} \gamma_{1}+\cdots+a_{l} \gamma_{l} \in \operatorname{Nef}(\boldsymbol{\phi}) \cap\left[x^{\prime}, x\right]_{\boldsymbol{e}}\left(a_{1}, \ldots, a_{l} \in \mathbb{R}_{\geq 0}\right.$ and $\left.a_{1}+\cdots+a_{l}=1\right)$, we have

$$
y=a_{1} y+\cdots+a_{l} y \geq_{\boldsymbol{e}} a_{1} \gamma_{1}+\cdots+a_{l} \gamma_{l}=v .
$$

This $y$ is actually the greatest element of $(-\infty, x]_{\boldsymbol{e}} \cap \operatorname{Nef}(\boldsymbol{\phi})$. Indeed, if $v \in$ $(-\infty, x]_{\boldsymbol{e}} \cap \operatorname{Nef}(\phi)$, then $\max \{v, y\} \in\left[x^{\prime}, x\right]_{\boldsymbol{e}} \cap \operatorname{Nef}(\boldsymbol{\phi})$ by Lemma 1.2.1, and hence

$$
v \leq \max \{v, y\} \leq y \text {. }
$$

Let us check the properties (a), (b) and (c). First of all, (a) is obvious. In order to see (b) and (c), we may assume that $z \neq 0$.

(b) Assume that $\phi_{\lambda}(y)>0$ for $\lambda \in \operatorname{Supp}(z ; \boldsymbol{e})$. Let $\epsilon$ be a sufficiently small positive number. Then $y+\epsilon e_{\lambda} \leq_{\boldsymbol{e}} x$ and

$$
\phi_{\mu}\left(y+\epsilon e_{\lambda}\right)=\phi_{\mu}(y)+\epsilon \phi_{\mu}\left(e_{\lambda}\right) \geq 0
$$

for all $\mu \in \Lambda$ because $0<\epsilon \ll 1$. Thus $y+\epsilon e_{\lambda} \in \operatorname{Nef}(\phi)$, which contradicts the maximality of $y$. Therefore, $\phi_{\lambda}(y)=0$ for $\lambda \in \operatorname{Supp}(z ; \boldsymbol{e})$.

(c) Assume that there is $v \in\left(\sum_{\lambda \in \operatorname{Supp}(z ; \boldsymbol{e})} \mathbb{R}_{\geq 0} e_{\lambda}\right) \backslash\{0\}$ such that $\phi_{\lambda}(v) \geq 0$ for all $\lambda \in \operatorname{Supp}(z ; \boldsymbol{e})$. Then there is a sufficiently small positive number $\epsilon^{\prime}$ such that $y+\epsilon^{\prime} v \leq_{\boldsymbol{e}} x$. Note that $\phi_{\mu}\left(y+\epsilon^{\prime} v\right) \geq 0$ for all $\mu$, which yields a contradiction as before.

Lemma 1.2.3. Let $W$ be a vector space over $\mathbb{R}$. Let $e_{1}, \ldots, e_{n} \in W$ and $\phi_{1}, \ldots, \phi_{n}$ $\in \operatorname{Hom}_{\mathbb{R}}(W, \mathbb{R})$ with the following properties:

(a) $\left\{\left(a_{1}, \ldots, a_{n}\right) \in \mathbb{R}_{\geq 0}^{n} \mid a_{1} e_{1}+\cdots+a_{n} e_{n}=0\right\}=\{(0, \ldots, 0)\}$.

(b) $\phi_{i}\left(e_{j}\right) \geq 0$ for all $i \neq j$.

(c) $\left\{x \in \mathbb{R}_{\geq 0} e_{1}+\cdots+\mathbb{R}_{\geq 0} e_{n} \mid \phi_{i}(x) \geq 0\right.$ for all $\left.i\right\}=\{0\}$.

Then we have the following:

(1) Let $Q$ be the $(n \times n)$-matrix $\left(\phi_{i}\left(e_{j}\right)\right)$. Then there are $(n \times n)$-matrices $A$ and $B$ with the following properties:

(1.1) A (resp. B) is a lower (resp. upper) triangular matrix consisting of nonnegative numbers.

(1.2) $\operatorname{det} A>0, \operatorname{det} B>0$ and

$$
A Q B=\left(\begin{array}{ccc}
-1 & \cdots & 0 \\
\vdots & \ddots & \vdots \\
0 & \cdots & -1
\end{array}\right)
$$

(1.3) If $Q$ is symmetric, then $B={ }^{t} A$. 
(2) The vectors $e_{1}, \ldots, e_{n}$ are linearly independent in

$$
W /\left\{x \in W \mid \phi_{1}(x)=\cdots=\phi_{n}(x)=0\right\} .
$$

Proof. (1) Let us begin with the following claim.

Claim 1.2.3.1. $\phi_{i}\left(e_{i}\right)<0$ for all $i$.

Proof. If $\phi_{i}\left(e_{i}\right) \geq 0$, then $e_{i} \in\left\{x \in \mathbb{R}_{\geq 0} e_{1}+\cdots+\mathbb{R}_{\geq 0} e_{n} \mid \phi_{j}(x) \geq 0\right.$ for all $\left.j\right\}$. This is a contradiction because $e_{i} \neq 0$.

The above claim proves (1) in the case where $n=1$. Now we set

$$
\phi_{i}^{\prime}=-\phi_{1}\left(e_{1}\right) \phi_{i}+\phi_{i}\left(e_{1}\right) \phi_{1}(i \geq 2), \quad e_{j}^{\prime}=-\phi_{1}\left(e_{1}\right) e_{j}+\phi_{1}\left(e_{j}\right) e_{1}(j \geq 2) .
$$

We claim the following:

Claim 1.2.3.2. (i) $\phi_{i}^{\prime}\left(e_{1}\right)=0$ and $\phi_{1}\left(e_{j}^{\prime}\right)=0$ for all $i \geq 2$ and $j \geq 2$.

(ii) $e_{2}^{\prime}, \ldots, e_{n}^{\prime}$ and $\phi_{2}^{\prime}, \ldots, \phi_{n}^{\prime}$ satisfy all assumptions (a)-(c) of the lemma.

(iii) Let $Q^{\prime}$ be the matrix $\left(\phi_{i}^{\prime}\left(e_{j}^{\prime}\right)\right)_{2 \leq i, j \leq n}$. Then

$$
A_{1} Q B_{1}=\left(\begin{array}{cc}
\phi_{1}\left(e_{1}\right) & 0 \\
0 & Q^{\prime}
\end{array}\right)
$$

where $A_{1}$ and $B_{1}$ are the matrices

$$
\left(\begin{array}{ccccc}
1 & 0 & 0 & \cdots & 0 \\
\phi_{2}\left(e_{1}\right) & -\phi_{1}\left(e_{1}\right) & 0 & \cdots & 0 \\
\phi_{3}\left(e_{1}\right) & 0 & -\phi_{1}\left(e_{1}\right) & \cdots & 0 \\
\vdots & \vdots & \vdots & \ddots & \vdots \\
\phi_{n}\left(e_{1}\right) & 0 & 0 & \cdots & -\phi_{1}\left(e_{1}\right)
\end{array}\right),\left(\begin{array}{ccccc}
1 & \phi_{1}\left(e_{2}\right) & \phi_{1}\left(e_{3}\right) & \cdots & \phi_{1}\left(e_{n}\right) \\
0 & -\phi_{1}\left(e_{1}\right) & 0 & \cdots & 0 \\
0 & 0 & -\phi_{1}\left(e_{1}\right) & \cdots & 0 \\
\vdots & \vdots & \vdots & \ddots & \vdots \\
0 & 0 & 0 & \cdots & -\phi_{1}\left(e_{1}\right)
\end{array}\right)
$$

respectively. Note that if $Q$ is symmetric, then $B_{1}={ }^{t} A_{1}$ and $Q^{\prime}$ is also symmetric.

Proof. (i) is obvious.

(ii) It is easy to see (a) for $e_{2}^{\prime}, \ldots, e_{n}^{\prime}$ by using Claim 1.2.3.1. For $i, j \geq 2$ with $i \neq j$, by Claim 1.2.3.1,

$$
\phi_{i}^{\prime}\left(e_{j}^{\prime}\right)=\phi_{1}\left(e_{1}\right)^{2} \phi_{i}\left(e_{j}\right)+\left(-\phi_{1}\left(e_{1}\right)\right) \phi_{i}\left(e_{1}\right) \phi_{1}\left(e_{j}\right) \geq 0 .
$$

Finally let $x \in \sum_{j \geq 2} \mathbb{R}_{\geq 0} e_{j}^{\prime}$ be such that $\phi_{i}^{\prime}(x) \geq 0$ for all $i \geq 2$. Note that $\phi_{i}^{\prime}(x)=\left(-\phi_{1}\left(e_{1}\right)\right) \phi_{i}(x)$ for $i \geq 2$. Therefore, $\phi_{i}(x) \geq 0$ for all $i \geq 1$, and hence $x=0$ because $\sum_{j \geq 2} \mathbb{R}_{\geq 0} e_{j}^{\prime} \subseteq \sum_{j \geq 1} \mathbb{R}_{\geq 0} e_{j}$.

(iii) is a straightforward calculation. 
We prove (1) by induction on $n$. By induction hypothesis, there are matrices $A^{\prime}$ and $B^{\prime}$ satisfying (1.1)-(1.3) for $Q^{\prime}$, that is,

$$
A^{\prime} Q^{\prime} B^{\prime}=\left(\begin{array}{ccc}
-1 & \cdots & 0 \\
\vdots & \ddots & \vdots \\
0 & \cdots & -1
\end{array}\right)
$$

Therefore,

$$
\left(\begin{array}{cc}
\frac{1}{\sqrt{-\phi_{1}\left(e_{1}\right)}} & 0 \\
0 & A^{\prime}
\end{array}\right) A_{1} Q B_{1}\left(\begin{array}{cc}
\frac{1}{\sqrt{-\phi_{1}\left(e_{1}\right)}} & 0 \\
0 & B^{\prime}
\end{array}\right)=\left(\begin{array}{ccc}
-1 & \cdots & 0 \\
\vdots & \ddots & \vdots \\
0 & \cdots & -1
\end{array}\right) .
$$

Thus (1) follows.

(2) Let $a_{1} e_{1}+\cdots+a_{n} e_{n}=0$ be a linear relation on

$$
W /\left\{x \in W \mid \phi_{1}(x)=\cdots=\phi_{n}(x)=0\right\} .
$$

Then there is $x \in W$ such that $x=a_{1} e_{1}+\cdots+a_{n} e_{n}$ and $\phi_{1}(x)=\cdots=\phi_{n}(x)=0$. Thus $0=\phi_{i}(x)=\sum \phi_{i}\left(e_{j}\right) a_{j}$. Hence (1) yields $(2)$.

Proof of Proposition 1.1.1. (1) Clearly (1.1) implies (1.2). If we assume (1.2), then (1.1) follows from Lemma 1.2.2.

(2) Let $x=y+z$ be a Zariski decomposition of $x$ with respect to $(\boldsymbol{e}, \boldsymbol{\phi})$ and $y^{\prime}=\max \left(\operatorname{Nef}_{\boldsymbol{\phi}} \cap(-\infty, x]_{\boldsymbol{e}}\right)$. Then $y \leq_{\boldsymbol{e}} y^{\prime}$. As $\phi_{\lambda}(y)=0$ for all $\lambda \in \operatorname{Supp}(z ; \boldsymbol{e})$,

$$
y^{\prime}-y \in\left\{x \in \sum_{\lambda \in \operatorname{Supp}(z ; \boldsymbol{e})} \mathbb{R}_{\geq 0} e_{\lambda} \mid \phi_{\lambda}(x) \geq 0 \text { for all } \lambda \in \operatorname{Supp}(z ; \boldsymbol{e})\right\},
$$

and hence $y^{\prime}=y$.

(3) follows from Lemma 1.2.3.

Remark 1.2.4. We assume that $\phi_{\lambda}\left(e_{\mu}\right) \in \mathbb{Q}$ for all $\lambda, \mu \in \Lambda$. Let $x \in \bigoplus_{\lambda} \mathbb{Q} e_{\lambda}$ be such that $(-\infty, x]_{\boldsymbol{e}} \cap \mathrm{Nef}_{\boldsymbol{\phi}} \neq \emptyset$. Let $x=y+z$ be the Zariski decomposition of $x$ with respect to $(\boldsymbol{e}, \boldsymbol{\phi})$. Then $y, z \in \bigoplus_{\lambda} \mathbb{Q} e_{\lambda}$. Indeed, if we set $\operatorname{Supp}(z ; \boldsymbol{e})=\left\{\lambda_{1}, \ldots, \lambda_{n}\right\}$ and $z=\sum a_{i} e_{\lambda_{i}}$, then

$$
\sum \phi_{\lambda_{i}}\left(e_{\lambda_{j}}\right) a_{j}=\phi_{\lambda_{i}}(x) \in \mathbb{Q}
$$

On the other hand, by our assumption and (3.1) in Proposition 1.1.1, we have $\left(\phi_{\lambda_{i}}\left(e_{\lambda_{j}}\right)\right)_{1 \leq i, j \leq n} \in \mathrm{GL}_{n}(\mathbb{Q})$. Thus $\left(a_{1}, \ldots, a_{n}\right) \in \mathbb{Q}^{n}$. 


\section{$\S 2$. Green functions for $\mathbb{R}$-Cartier divisors}

\section{$\S 2.1$. Plurisubharmonic functions}

Here we recall plurisubharmonic functions and the upper semicontinuous regularization of a function locally bounded above.

Let $T$ be a metric space with a metric $d$. A function $f: T \rightarrow\{-\infty\} \cup \mathbb{R}$ is said to be upper semicontinuous if $\{x \in T \mid f(x)<c\}$ is open for any $c \in \mathbb{R}$. In other words,

$$
f(a)=\limsup _{x \rightarrow a} f(x)\left(:=\inf _{\epsilon>0}(\sup \{f(y) \mid d(a, y) \leq \epsilon\})\right)
$$

for all $a \in T$. Let $u: T \rightarrow\{-\infty\} \cup \mathbb{R}$ be a function such that $u$ is locally bounded above. The upper semicontinuous regularization $u^{*}$ of $u$ is given by

$$
u^{*}(x)=\limsup _{y \rightarrow x} u(y)
$$

Note that $u^{*}$ is upper semicontinuous and $u \leq u^{*}$.

Let $D$ be an open set in $\mathbb{C}$. A function $u: D \rightarrow\{-\infty\} \cup \mathbb{R}$ is said to be subharmonic if $u$ is upper semicontinuous and

$$
u(a) \leq \frac{1}{2 \pi} \int_{0}^{2 \pi} u\left(a+r e^{\sqrt{-1} \theta}\right) d \theta
$$

for any $a \in D$ and $r \in \mathbb{R}_{>0}$ with $\{z \in \mathbb{C}|| z-a \mid \leq r\} \subseteq D$.

Let $X$ be a $d$-equidimensional complex manifold. A function $u: X \rightarrow\{-\infty\} \cup \mathbb{R}$ is said to be plurisubharmonic if $u$ is upper semicontinuous and $u \circ \phi$ is subharmonic for any analytic map $\phi:\{z \in \mathbb{C}|| z \mid<1\} \rightarrow X$. We say $u$ is a real valued plurisubharmonic function if $u(x) \neq-\infty$ for all $x \in X$. If $X$ is an open set in $\mathbb{C}^{d}$, then an upper semicontinuous function $u: X \rightarrow \mathbb{R} \cup\{-\infty\}$ is plurisubharmonic if and only if

$$
u(a) \leq \frac{1}{2 \pi} \int_{0}^{2 \pi} u(a+\xi \exp (\sqrt{-1} \theta)) d \theta
$$

for any $a \in X$ and $\xi \in \mathbb{C}^{d}$ with $\{a+\xi \exp (\sqrt{-1} \theta) \mid 0 \leq \theta \leq 2 \pi\} \subseteq X$. As an example, if $f_{1}, \ldots, f_{r}$ are holomorphic functions on $X$, then

$$
\log \left(\left|f_{1}\right|^{2}+\cdots+\left|f_{r}\right|^{2}\right)
$$

is a plurisubharmonic function on $X$. In particular, if

$$
x \notin\left\{z \in X \mid f_{1}(z)=\cdots=f_{r}(z)=0\right\},
$$

then $d d^{c}\left(\log \left(\left|f_{1}\right|^{2}+\cdots+\left|f_{r}\right|^{2}\right)\right)$ is semipositive around $x$. 
Let $\left\{u_{\lambda}\right\}_{\lambda \in \Lambda}$ be a family of plurisubharmonic functions on $X$, locally uniformly bounded above. If we set $u(x):=\sup _{\lambda \in \Lambda} u_{\lambda}(x)$ for $x \in X$, then the upper semicontinuous regularization $u^{*}$ of $u$ is plurisubharmonic and $u=u^{*}$ (a.e.) (cf. [9, Theorem 2.9.14 and Proposition 2.6.2]). Moreover, let $\left\{v_{n}\right\}_{n=1}^{\infty}$ be a sequence of plurisubharmonic functions on $X$, locally uniformly bounded above. If we set $v(x):=\lim \sup _{n \rightarrow \infty} v_{n}(x)$ for $x \in X$, then the upper semicontinuous regularization $v^{*}$ of $v$ is plurisubharmonic and $v=v^{*}$ (a.e.) (cf. [9, Proposition 2.9.17 and Theorem 2.6.3]).

\section{$\S 2.2 . \mathbb{R}$-Cartier divisors}

Let $X$ be either a $d$-equidimensional smooth algebraic variety over $\mathbb{C}$, or a $d$-equidimensional complex manifold. Let $\operatorname{Div}(X)$ be the group of Cartier divisors on $X$. An element $D$ of $\operatorname{Div}(X)_{\mathbb{R}}:=\operatorname{Div}(X) \otimes_{\mathbb{Z}} \mathbb{R}$ is called an $\mathbb{R}$-Cartier divisor on $X$. Let $D=\sum_{i=1}^{n} a_{i} D_{i}$ be the irreducible decomposition of $D$, that is, $a_{1}, \ldots, a_{n} \in \mathbb{R}$ and $D_{i}$ 's are reduced and irreducible divisors on $X$. For a prime divisor $\Gamma$ on $X$ (i.e., a reduced and irreducible divisor on $X$ ), the coefficient of $\Gamma$ in the above irreducible decomposition is denoted by $\operatorname{mult}_{\Gamma}(D)$, that is,

$$
\operatorname{mult}_{\Gamma}(D)= \begin{cases}a_{i} & \text { if } \Gamma=D_{i} \text { for some } i, \\ 0 & \text { if } \Gamma \neq D_{i} \text { for all } i,\end{cases}
$$

and $D=\sum_{\Gamma} \operatorname{mult}_{\Gamma}(D) \Gamma$. The support $\operatorname{Supp}(D)$ of $D$ is defined by $\bigcup_{\text {mult }}(D) \neq 0$ If $a_{i} \geq 0$ for all $i$, then $D$ is said to be effective, written $D \geq 0$. More generally, for $D_{1}, D_{2} \in \operatorname{Div}(X)_{\mathbb{R}}$,

$$
D_{1} \leq D_{2}\left(\text { or } D_{2} \geq D_{2}\right) \stackrel{\text { def }}{\Longleftrightarrow} D_{2}-D_{1} \geq 0 .
$$

The round-up $\lceil D\rceil$ of $D$ and the round-down $\lfloor D\rfloor$ of $D$ are defined by

$$
\lceil D\rceil=\sum_{i=1}^{n}\left\lceil a_{i}\right\rceil D_{i} \quad \text { and } \quad\lfloor D\rfloor=\sum_{i=1}^{n}\left\lfloor a_{i}\right\rfloor D_{i},
$$

where $\lceil x\rceil=\min \{a \in \mathbb{Z} \mid x \leq a\}$ and $\lfloor x\rfloor=\max \{a \in \mathbb{Z} \mid a \leq x\}$ for $x \in \mathbb{R}$.

We assume that $X$ is algebraic. Let $\operatorname{Rat}(X)$ be the ring of rational functions on $X$. Note that $X$ is not necessarily connected, so that $\operatorname{Rat}(X)$ is not necessarily a field. In the case where $X$ is connected, we define

$$
H^{0}(X, D):=\left\{\phi \in \operatorname{Rat}(X)^{\times} \mid(\phi)+D \geq 0\right\} \cup\{0\} .
$$

In general, let $X=\amalg_{\alpha} X_{\alpha}$ be the decomposition into connected components, and let $D_{\alpha}=\left.D\right|_{X_{\alpha}}$. Then we define

$$
H^{0}(X, D):=\bigoplus_{\alpha} H^{0}\left(X_{\alpha}, D_{\alpha}\right) .
$$


Note that if $D$ is effective, then $H^{0}(X, D)$ is generated by

$$
\left\{\phi \in \operatorname{Rat}(X)^{\times} \mid(\phi)+D \geq 0\right\} .
$$

Indeed, for $\phi_{\alpha} \in H^{0}\left(X_{\alpha}, D_{\alpha}\right)$, if we choose $c \in \mathbb{C}^{\times}$with $\phi_{\alpha}+c \neq 0$, then

$$
\left(0, \ldots, 0, \phi_{\alpha}, 0, \ldots, 0\right)=\left(1, \ldots, 1, \phi_{\alpha}+c, 1, \ldots, 1\right)-(1, \ldots, 1, c, 1, \ldots, 1),
$$

which shows the assertion. Since

$$
\left(\phi_{\alpha}\right)+D_{\alpha} \geq 0 \Leftrightarrow\left(\phi_{\alpha}\right)+\left\lfloor D_{\alpha}\right\rfloor \geq 0,
$$

we have $H^{0}(X, D)=H^{0}(X,\lfloor D\rfloor)$.

In the case where $X$ is not necessarily algebraic, the ring of meromorphic functions on $X$ is denoted by $\mathcal{M}(X)$. By using $\mathcal{M}(X)$ instead of $\operatorname{Rat}(X)$, we can define $H_{\mathcal{M}}^{0}(X, D)$ in the same way as above, that is, if $X$ is connected, then

$$
H_{\mathcal{M}}^{0}(X, D):=\left\{\phi \in \mathcal{M}(X)^{\times} \mid(\phi)+D \geq 0\right\} \cup\{0\} .
$$

If $X$ is a proper smooth algebraic scheme over $\mathbb{C}$, then $\operatorname{Rat}(X)=\mathcal{M}(X)$ by GAGA, and hence $H^{0}(X, D)=H_{\mathcal{M}}^{0}(X, D)$.

\section{§2.3. Definition of Green functions for $\mathbb{R}$-Cartier divisors}

Let $X$ be a $d$-equidimensional complex manifold. Let $\mathcal{L}_{\text {loc }}^{1}$ be the sheaf of locally integrable functions, that is,

$$
\mathcal{L}_{\text {loc }}^{1}(U):=\{g: U \rightarrow \mathbb{R} \cup\{ \pm \infty\} \mid g \text { is locally integrable }\}
$$

for an open set $U$ of $X$. Let $\mathscr{T}$ be a subsheaf of $\mathcal{L}_{\text {loc }}^{1}$ and let $S$ be a subset of $\mathbb{R} \cup\{ \pm \infty\}$. Then $\mathscr{T}_{S}, \mathscr{T}^{b}$ and $-\mathscr{T}$ are defined as follows:

$$
\begin{aligned}
\mathscr{T}_{S}(U) & :=\{g \in \mathscr{T}(U) \mid g(x) \in S \text { for all } x \in U\}, \\
\mathscr{T}^{b}(U) & :=\{g \in \mathscr{T}(U) \mid g \text { is locally bounded on } U\}, \\
-\mathscr{T}(U) & :=\left\{-g \in \mathcal{L}_{\text {loc }}^{1}(U) \mid g \in \mathscr{T}(U)\right\} .
\end{aligned}
$$

Let $\mathscr{T}^{\prime}$ be another subsheaf of $\mathcal{L}_{\text {loc }}^{1}$. We assume that $u+u^{\prime}$ is well-defined as a function for any open set $U, u \in \mathscr{T}(U)$ and $u^{\prime} \in \mathscr{T}^{\prime}(U)$. Then $\mathscr{T}+\mathscr{T}^{\prime}$ is defined by

$$
\left(\mathscr{T}+\mathscr{T}^{\prime}\right)(U):=\left\{\begin{array}{l|l}
g \in \mathcal{L}_{\text {loc }}^{1}(U) & \begin{array}{l}
\text { For any } x \in U, \text { we can find an open } \\
\text { neighborhood } V_{x}, u \in \mathscr{T}\left(V_{x}\right) \text { and } \\
u^{\prime} \in \mathscr{T}^{\prime}\left(V_{x}\right) \text { such that }\left.g\right|_{V_{x}}=u+u^{\prime}
\end{array}
\end{array}\right\} .
$$


Similarly, if $u-u^{\prime}$ is well-defined as a function for any open set $U, u \in \mathscr{T}(U)$ and $u^{\prime} \in \mathscr{T}^{\prime}(U)$, then $\mathscr{T}-\mathscr{T}^{\prime}$ is defined by

$$
\left(\mathscr{T}-\mathscr{T}^{\prime}\right)(U):=\left\{\begin{array}{l|l}
g \in \mathcal{L}_{\text {loc }}^{1}(U) & \begin{array}{l}
\text { For any } x \in U, \text { we can find an open } \\
\text { neighborhood } V_{x}, u \in \mathscr{T}\left(V_{x}\right) \text { and } \\
u^{\prime} \in \mathscr{T}^{\prime}\left(V_{x}\right) \text { such that }\left.g\right|_{V_{x}}=u-u^{\prime}
\end{array}
\end{array}\right\} .
$$

Note that $\mathscr{T}-\mathscr{T}^{\prime}=\mathscr{T}+\left(-\mathscr{T}^{\prime}\right)$. A subsheaf $\mathscr{T}$ of $\mathcal{L}_{\text {loc }}^{1}$ is called a type for Green functions on $X$ if the following conditions are satisfied (here $U$ is an arbitrary open set of $X)$ :

(1) If $u, v \in \mathscr{T}(U)$ and $a \in \mathbb{R}_{\geq 0}$, then $u+v \in \mathscr{T}(U)$ and $a u \in \mathscr{T}(U)$.

(2) If $u, v \in \mathscr{T}(U)$ and $u \leq v$ (a.e.), then $u \leq v$.

(3) If $\phi \in \mathscr{O}_{X}^{\times}(U)$ (i.e., $\phi$ is a nowhere vanishing holomorphic function on $U$ ), then $\log |\phi|^{2} \in \mathscr{T}(U)$.

Note that, for $u, v \in \mathscr{T}(U), u=v$ if $u=v$ (a.e.). If $\mathscr{T}=\mathscr{T}_{\mathbb{R}}$, that is, $u(x) \in \mathbb{R}$ for any open set $U, u \in \mathscr{T}(U)$ and $x \in U$, then $\mathscr{T}$ is called a real valued type. As examples of types for Green functions on $X$, we have

$C^{0}$ : the sheaf of continuous functions,

$C^{\infty}$ : the sheaf of $C^{\infty}$-functions,

PSH: the sheaf of plurisubharmonic functions.

Note that

$$
\operatorname{PSH}_{\mathbb{R}}(U)=\{g \in \operatorname{PSH}(U) \mid g(x) \neq-\infty \text { for all } x \in U\}
$$

for an open set $U$ of $X$. Let $\mathscr{T}$ and $\mathscr{T}^{\prime}$ be types for Green functions on $X$. We say $\mathscr{T}^{\prime}$ is a subjacent type of $\mathscr{T}$ if the following property holds for any open set $U$ of $X$ :

$$
u^{\prime} \leq u \text { (a.e.) on } U \text { for } u^{\prime} \in \mathscr{T}^{\prime}(U) \text { and } u \in \mathscr{T}(U) \Rightarrow u^{\prime} \leq u \text { on } U \text {. }
$$

Lemma 2.3.1. Let $\mathscr{T}$ be either $C^{0}+\mathrm{PSH}$ or $C^{0}+\mathrm{PSH}_{\mathbb{R}}-\mathrm{PSH}_{\mathbb{R}}$. Then $\mathscr{T}$ is a type for Green functions on X. Moreover, PSH is a subjacent type of $\mathscr{T}$.

Proof. The conditions (1) and (3) are obvious for $\mathscr{T}$. Let us see (2). For $z=$ $\left(z_{1}, \ldots, z_{d}\right) \in \mathbb{C}^{d}$, we set $\|z\|=\sqrt{\left|z_{1}\right|^{2}+\cdots+\left|z_{d}\right|^{2}}$. Moreover, for $a \in \mathbb{C}^{d}$ and $r>0$, the ball $\left\{z \in \mathbb{C}^{d} \mid\|z-a\|<r\right\}$ is denoted by $B^{d}(a ; r)$.

The assertion of (2) is local, so that we may assume that $X=B^{d}((0, \ldots, 0) ; 1)$. It is sufficient to see that, for $u_{1}, u_{2} \in \mathscr{T}(X)$, if $u_{1} \leq u_{2}$ (a.e.), then $u_{1} \leq u_{2}$. Let us fix $a \in B^{d}((0, \ldots, 0) ; 1)$. There are a sufficiently small $r>0$ and $v_{i j} \in$ $\mathcal{L}_{\text {loc }}^{1}\left(B^{d}(a ; r)\right)(i=1,2$ and $j=1,2,3)$ with the following properties: 
(a) $u_{1}=v_{11}+v_{12}-v_{13}$ and $u_{2}=v_{21}+v_{22}-v_{23}$.

(b) $v_{11}, v_{21} \in C^{0}\left(B^{d}(a ; r)\right)$.

(c) $v_{12}, v_{22} \in \operatorname{PSH}\left(B^{d}(a ; r)\right)$ in the case $\mathscr{T}=C^{0}+\mathrm{PSH}$.

$(\text { c) })^{\prime} v_{12}, v_{22} \in \mathrm{PSH}_{\mathbb{R}}\left(B^{d}(a ; r)\right)$ in the case $\mathscr{T}=C^{0}+\mathrm{PSH}_{\mathbb{R}}-\mathrm{PSH}_{\mathbb{R}}$.

(d) $v_{13}=v_{23}=0$ in the case $\mathscr{T}=C^{0}+\mathrm{PSH}$.

$(\mathrm{d})^{\prime} v_{13}, v_{23} \in \mathrm{PSH}_{\mathbb{R}}\left(B^{d}(a ; r)\right)$ in the case $\mathscr{T}=C^{0}+\mathrm{PSH}_{\mathbb{R}}-\mathrm{PSH}_{\mathbb{R}}$.

Let $\chi_{\epsilon}(\epsilon>0)$ be the standard smoothing kernels on $\mathbb{C}^{d}$ (cf. [9, Section 2.5]). It is well known that $v_{i j}(a)=\lim _{\epsilon \rightarrow 0}\left(v_{i j} * \chi_{\epsilon}\right)(a)$ for $i=1,2$ and $j=1,2,3$ (cf. [9, Proposition 2.5.2 and Theorem 2.9.2]). In the case $\mathscr{T}=C^{0}+\mathrm{PSH}$, since $v_{11}(a), v_{21}(a) \in \mathbb{R}, v_{12}(a), v_{22}(a) \in \mathbb{R} \cup\{-\infty\}$ and $v_{13}=v_{23}=0$,

$$
\begin{aligned}
\lim _{\epsilon \rightarrow 0}\left(u_{i} * \chi_{\epsilon}\right)(a) & =\lim _{\epsilon \rightarrow 0}\left(\left(v_{i 1} * \chi_{\epsilon}\right)(a)+\left(v_{i 2} * \chi_{\epsilon}\right)(a)-\left(v_{i 3} * \chi_{\epsilon}\right)(a)\right) \\
& =\lim _{\epsilon \rightarrow 0}\left(v_{i 1} * \chi_{\epsilon}\right)(a)+\lim _{\epsilon \rightarrow 0}\left(v_{i 2} * \chi_{\epsilon}\right)(a)-\lim _{\epsilon \rightarrow 0}\left(v_{i 3} * \chi_{\epsilon}\right)(a) \\
& =v_{i 1}(a)+v_{i 2}(a)-v_{i 3}(a)=u_{i}(a) .
\end{aligned}
$$

If $\mathscr{T}=C^{0}+\mathrm{PSH}_{\mathbb{R}}-\mathrm{PSH}_{\mathbb{R}}$, then, in the same way as above, we can also see $u_{i}(a)=\lim _{\epsilon \rightarrow 0}\left(u_{i} * \chi_{\epsilon}\right)(a)$ for $i=1,2$ because $v_{i j}(a) \in \mathbb{R}$ for $i=1,2$ and $j=1,2,3$. Therefore, (2) follows from the inequalities $\left(u_{1} * \chi_{\epsilon}\right)(a) \leq\left(u_{2} * \chi_{\epsilon}\right)(a)$ (for all $\epsilon>0$ ). The last assertion can be checked similarly.

Let $\mathscr{T}$ be a type for Green functions on $X$. Let $g$ be a locally integrable function on $X$ and let $D=\sum_{i=1}^{l} a_{i} D_{i}$ be an $\mathbb{R}$-Cartier divisor on $X$, where $D_{i}$ 's are reduced and irreducible divisors on $X$. We say $g$ is a D-Green function of $\mathscr{T}$-type (or a Green function of $\mathscr{T}$-type for $D$ ) if, for each point $x \in X, g$ has a local expression

$$
g=u+\sum_{i=1}^{l}\left(-a_{i}\right) \log \left|f_{i}\right|^{2} \quad \text { (a.e.) }
$$

over an open neighborhood $U_{x}$ of $x$ such that $u \in \mathscr{T}\left(U_{x}\right)$, where $f_{1}, \ldots, f_{l}$ are local equations of $D_{1}, \ldots, D_{l}$ on $U_{x}$ respectively. Note that this definition does not depend on the choice of local equations $f_{1}, \ldots, f_{l}$ on $U_{x}$ by the properties (1) and (3) of $\mathscr{T}$. The set of all $D$-Green functions of $\mathscr{T}$-type is denoted by $G_{\mathscr{T}}(X ; D)$.

Let $g$ be a $D$-Green function of $\mathscr{T}$-type. We say $g$ is of upper bounded type (resp. of lower bounded type) if, in the above local expression around each point of $X, u$ is locally bounded above (resp. locally bounded below). If $g$ is of upper and lower bounded type, then $g$ is said to be of bounded type. These definitions also do not depend on the choice of local equations. Note that the set of all $D$-Green functions of $\mathscr{T}$-type and of bounded type is nothing other than $G_{\mathscr{T}^{b}}(X ; D)$. 
We assume $x \notin \operatorname{Supp}(D)$. Let $g$ be a $D$-Green function of $\mathscr{T}$-type. Let $f_{1}, \ldots, f_{l}$ and $f_{1}^{\prime}, \ldots, f_{l}^{\prime}$ be two sets of local equations of $D_{1}, \ldots, D_{l}$ on an open neighborhood $U_{x}$ of $x$. Let

$$
g=u+\sum_{i=1}^{l}\left(-a_{i}\right) \log \left|f_{i}\right|^{2} \text { (a.e.) and } g=u^{\prime}+\sum_{i=1}^{l}\left(-a_{i}\right) \log \left|f_{i}^{\prime}\right|^{2} \text { (a.e.) }
$$

be two local expressions of $g$ over $U_{x}$, where $u, u^{\prime} \in \mathscr{T}\left(U_{x}\right)$. Since $x \notin \operatorname{Supp}(D)$, there is an open neighborhood $V_{x}$ of $x$ such that $V_{x} \subseteq U_{x}$ and $f_{1}, \ldots, f_{l}, f_{1}^{\prime}, \ldots, f_{l}^{\prime} \in$ $\mathscr{O}_{X}^{\times}\left(V_{x}\right)$. Thus, by the properties (1) and (3) of $\mathscr{T}$,

$$
u+\sum_{i=1}^{l}\left(-a_{i}\right) \log \left|f_{i}\right|^{2}, \quad u^{\prime}+\sum_{i=1}^{l}\left(-a_{i}\right) \log \left|f_{i}^{\prime}\right|^{2} \in \mathscr{T}\left(V_{x}\right),
$$

and hence

$$
u+\sum_{i=1}^{l}\left(-a_{i}\right) \log \left|f_{i}\right|^{2}=u^{\prime}+\sum_{i=1}^{l}\left(-a_{i}\right) \log \left|f_{i}^{\prime}\right|^{2} \in \mathscr{T}\left(V_{x}\right)
$$

over $V_{x}$ by the second property of $\mathscr{T}$. This observation shows that

$$
u(x)+\sum_{i=1}^{l}\left(-a_{i}\right) \log \left|f_{i}(x)\right|^{2}
$$

does not depend on the choice of the local expression of $g$. In this sense, the value

$$
u(x)+\sum_{i=1}^{l}\left(-a_{i}\right) \log \left|f_{i}(x)\right|^{2}
$$

is called the canonical value of $g$ at $x$ and it is denoted by $g_{\text {can }}(x)$. Note that $g_{\text {can }} \in \mathscr{T}(X \backslash \operatorname{Supp}(D))$ and $g=g_{\text {can }}$ (a.e.) on $X \backslash \operatorname{Supp}(D)$. Moreover, if $\mathscr{T}$ is real valued, then $g_{\text {can }}(x) \in \mathbb{R}$. It is easy to prove the following propositions.

Proposition 2.3.2. Let $g$ be a D-Green function of $C^{\infty}$-type. Then the current $d d^{c}([g])+\delta_{D}$ is represented by a unique $C^{\infty}$-form $\alpha$, that is, $d d^{c}([g])+\delta_{D}=[\alpha]$. We often identify the current $d d^{c}([g])+\delta_{D}$ with $\alpha$, and denote it by $c_{1}(D, g)$.

Proposition 2.3.3. Let $\mathscr{T}^{\prime}$ and $\mathscr{T}^{\prime \prime}$ be two types for Green functions on $X$ such that $\mathscr{T}^{\prime}, \mathscr{T}^{\prime \prime} \subseteq \mathscr{T}$. Then $G_{\mathscr{T}^{\prime} \cap \mathscr{T}^{\prime \prime}}(X ; D)=G_{\mathscr{T}^{\prime}}(X ; D) \cap G_{\mathscr{T}^{\prime \prime}}(X ; D)$.

Proposition 2.3.4. (1) If $g$ is a D-Green function of $\mathscr{T}$-type and $a \in \mathbb{R}_{\geq 0}$, then ag is an (aD)-Green function of $\mathscr{T}$-type. Moreover, if $x \notin \operatorname{Supp}(D)$, then $(a g)_{\operatorname{can}}(x)=a g_{\text {can }}(x)$.

(2) If $g_{1}$ (resp. $\left.g_{2}\right)$ is a $D_{1}$-Green function of $\mathscr{T}$-type (resp. $D_{2}$-Green function of $\mathscr{T}$-type), then $g_{1}+g_{2}$ is a $\left(D_{1}+D_{2}\right)$-Green function of $\mathscr{T}$-type. Moreover, if $x \notin \operatorname{Supp}\left(D_{1}\right) \cup \operatorname{Supp}\left(D_{2}\right)$, then $\left(g_{1}+g_{2}\right)_{\text {can }}(x)=\left(g_{1}\right)_{\text {can }}(x)+\left(g_{2}\right)_{\text {can }}(x)$. 
(3) Assume that $-\mathscr{T} \subseteq \mathscr{T}$. If $g$ is a D-Green function of $\mathscr{T}$-type, then $-g$ is a $(-D)$-Green function of $\mathscr{T}$-type. Moreover, if $x \notin \operatorname{Supp}(D)$, then $(-g)_{\operatorname{can}}(x)=$ $-g_{\text {can }}(x)$.

(4) Let $g$ be a $D$-Green function of $\mathscr{T}$-type. If $g \geq 0$ (a.e.) and $x \notin \operatorname{Supp}(D)$, then $g_{\text {can }}(x) \geq 0$.

Finally, we prove three propositions.

Proposition 2.3.5. Let $D=b_{1} E_{1}+\cdots+b_{r} E_{r}$ be an $\mathbb{R}$-Cartier divisor on $X$ such that $b_{1}, \ldots, b_{r} \in \mathbb{R}$ and $E_{i}$ 's are Cartier divisors on $X$. Let $g$ be a D-Green function of $\mathscr{T}$-type on $X$. Let $U$ be an open set of $X$ and let $\phi_{1}, \ldots, \phi_{r}$ be local equations of $E_{1}, \ldots, E_{r}$ over $U$ respectively. Then there is a unique expression

$$
\left.g=u+\sum_{i=1}^{r}\left(-b_{i}\right) \log \left|\phi_{i}\right|^{2} \quad \text { (a.e. }\right) \quad(u \in \mathscr{T}(U))
$$

on $U$ modulo null functions. This expression is called the local expression of $g$ over $U$ with respect to $\phi_{1}, \ldots, \phi_{r}$.

Proof. Let us choose reduced and irreducible divisors $D_{1}, \ldots, D_{l}$ and $\alpha_{i j} \in \mathbb{Z}$ such that $E_{i}=\sum_{j=1}^{l} \alpha_{i j} D_{j}$ for each $i$. If we set $a_{j}=\sum_{i=1}^{r} b_{i} \alpha_{i j}$, then $D=\sum_{j=1}^{l} a_{j} D_{j}$. For each point $x \in U$, there are an open neighborhood $U_{x}$ of $x$, local equations $f_{1, x}, \ldots, f_{l, x}$ of $D_{1}, \ldots, D_{l}$ on $U_{x}$ and $u_{x} \in \mathscr{T}\left(U_{x}\right)$ such that $U_{x} \subseteq U$ and

$$
g=u_{x}+\sum_{j=1}^{l}\left(-a_{j}\right) \log \left|f_{j, x}\right|^{2} \quad \text { (a.e.) }
$$

on $U_{x}$. Note that

$$
g=u_{x}+\sum_{i=1}^{r}\left(-b_{i}\right) \log \left|\prod_{j=1}^{l} f_{j, x}^{\alpha_{i j}}\right|^{2} \quad \text { (a.e.) }
$$

and $\prod_{j=1}^{l} f_{j, x}^{\alpha_{i j}}$ is a local equation of $E_{i}$ over $U_{x}$, so that we can find nowhere vanishing holomorphic functions $e_{1, x}, \ldots, e_{r, x}$ on $U_{x}$ such that $\prod_{j=1}^{l} f_{j, x}^{\alpha_{i j}}=e_{i, x} \phi_{i}$ on $U_{x}$ for all $i=1, \ldots, r$. Then

$$
g=u_{x}+\sum_{i=1}^{r}\left(-b_{i}\right) \log \left|e_{i, x}\right|^{2}+\sum_{i=1}^{r}\left(-b_{i}\right) \log \left|\phi_{i}\right|^{2} \quad \text { (a.e.) }
$$

on $U_{x}$. Thus, for $x, x^{\prime} \in U$,

$$
u_{x}+\sum_{i=1}^{r}\left(-b_{i}\right) \log \left|e_{i, x}\right|^{2}=u_{x^{\prime}}+\sum_{i=1}^{r}\left(-b_{i}\right) \log \left|e_{i, x^{\prime}}\right|^{2} \quad \text { (a.e.) }
$$


on $U_{x} \cap U_{x^{\prime}}$, and hence

$$
u_{x}+\sum_{i=1}^{r}\left(-b_{i}\right) \log \left|e_{i, x}\right|^{2}=u_{x^{\prime}}+\sum_{i=1}^{r}\left(-b_{i}\right) \log \left|e_{i, x^{\prime}}\right|^{2}
$$

on $U_{x} \cap U_{x^{\prime}}$. This means that there is $u \in \mathscr{T}(U)$ such that $u$ is locally given by $u_{x}+\sum_{i=1}^{r}\left(-b_{i}\right) \log \left|e_{i, x}\right|^{2}$. Therefore, $g=u+\sum_{i=1}^{r}\left(-b_{i}\right) \log \left|\phi_{i}\right|^{2}$ (a.e.) on $U$. The uniqueness of the expression modulo null functions is obvious by the second property of $\mathscr{T}$.

Proposition 2.3.6. Let $g$ be a D-Green function of $\mathscr{T}$-type. Then we have the following:

(1) If $g$ is of lower bounded type, then locally $|\phi| \exp (-g / 2)$ is essentially bounded above for $\phi \in H_{\mathcal{M}}^{0}(X, D)$.

(2) If $g$ is of upper bounded type, then there is a D-Green function $g^{\prime}$ of $C^{\infty}$-type such that $g \leq g^{\prime}$ (a.e.).

Proof. We set $D=\sum_{i=1}^{l} a_{i} D_{i}$ such that $a_{1}, \ldots, a_{l} \in \mathbb{R}$ and $D_{i}$ 's are reduced and irreducible divisors on $X$.

(1) Clearly we may assume that $X$ is connected. For $x \in X$, let

$$
g=u+\sum_{i=1}^{l}\left(-a_{i}\right) \log \left|f_{i}\right|^{2} \quad \text { (a.e.) }
$$

be a local expression of $g$ around $x$, where $f_{1}, \ldots, f_{l}$ are local equations of $D_{1}, \ldots$, $D_{l}$. For $\phi \in H_{\mathcal{M}}^{0}(X, D)$, we set $\phi=f_{1}^{b_{1}} \cdots f_{l}^{b_{l}} \cdot v$ around $x$ where $v$ has no factors of $f_{1}, \ldots, f_{l}$. Then, as $(\phi)+D \geq 0$, we can see that $a_{i}+b_{i} \geq 0$ for all $i$, and that $v$ is a holomorphic function around $x$. On the other hand,

$$
\exp (-g / 2)|\phi|=\exp (-u / 2)\left|f_{1}\right|^{a_{1}+b_{1}} \cdots\left|f_{n}\right|^{a_{n}+b_{n}}|v| \quad \text { (a.e.) }
$$

as required.

(2) By our assumption, there is a locally finite open covering $\left\{U_{\lambda}\right\}_{\lambda \in \Lambda}$ with the following properties:

(a) There are local equations $f_{\lambda, 1}, \ldots, f_{\lambda, n}$ of $D_{1}, \ldots, D_{n}$ on $U_{\lambda}$.

(b) There is a constant $C_{\lambda}$ such that $g \leq C_{\lambda}-\sum a_{i} \log \left|f_{\lambda, i}\right|^{2}$ (a.e.) on $U_{\lambda}$.

Let $\left\{\rho_{\lambda}\right\}_{\lambda \in \Lambda}$ be a partition of unity subordinate to the covering $\left\{U_{\lambda}\right\}_{\lambda \in \Lambda}$. We set

$$
g^{\prime}=\sum_{\lambda \in \lambda} \rho_{\lambda}\left(C_{\lambda}-\sum a_{i} \log \left|f_{\lambda, i}\right|^{2}\right) .
$$

Clearly $g \leq g^{\prime}$ (a.e.). Moreover, by Lemma 2.4.1 below, $g^{\prime}$ is a $D$-Green function of $C^{\infty}$-type. 
Proposition 2.3.7. Let $g$ be a D-Green function of $\left(\mathrm{PSH}+C^{\infty}\right)$-type. Let $A$ be an $\mathbb{R}$-Cartier divisor on $X$, and let $h$ be an $A$-Green function of $C^{\infty}$-type. Let $\alpha=c_{1}(A, h)$, that is, $\alpha$ is a $C^{\infty}(1,1)$-form on $X$ such that $d d^{c}([h])+\delta_{A}=[\alpha]$ (cf. Proposition 2.3.2). If $X$ is compact and $\alpha$ is positive, then there is a positive number $t_{0}$ such that $g+$ th is a $(D+t A)$-Green function of PSH-type for all $t \in \mathbb{R}_{\geq t_{0}}$.

Proof. For each $x \in X$, let

$$
g=u_{x}+\sum_{i}\left(-a_{i}\right) \log \left|f_{i}\right|^{2} \quad \text { (a.e.), } \quad h=v_{x}+\sum_{i}\left(-b_{i}\right) \log \left|f_{i}\right|^{2} \quad \text { (a.e.) }
$$

be local expressions of $g$ and $h$ respectively over an open neighborhood $U_{x}$ of $x$. By our assumption, after shrinking $U_{x}$ if necessary, there are a plurisubharmonic function $p_{x}$ and a $C^{\infty}$-function $q_{x}$ such that $u_{x}=p_{x}+q_{x}$. Moreover, since $\alpha$ is positive, shrinking $U_{x}$ if necessary, we can find a positive number $t_{x}$ such that $d d^{c}\left(q_{x}\right)+t \alpha$ is positive for all $t \geq t_{x}$. By the compactness of $X$, we can choose finitely many $x_{1}, \ldots, x_{r} \in X$ such that $X=U_{x_{1}} \cup \cdots \cup U_{x_{r}}$. If we set $t_{0}=$ $\max \left\{t_{x_{1}}, \ldots, t_{x_{r}}\right\}$, then, for $t \geq t_{0}$,

$$
g+t h=p_{x_{j}}+\left(q_{x_{j}}+t v_{x_{j}}\right)+\sum_{i}-\left(a_{i}+t b_{i}\right) \log \left|f_{i}\right|^{2} \quad \text { (a.e.) }
$$

over $U_{x_{j}}$. Note that $d d^{c}\left(q_{x_{j}}+t v_{x_{j}}\right)=d d^{c}\left(q_{x_{j}}\right)+t \alpha$ is positive, which means that $q_{x_{j}}+t v_{x_{j}}$ is a $C^{\infty}$-plurisubharmonic function. Thus $g+t h$ is of PSH-type.

\section{§2.4. Partitions of Green functions}

Let $X$ be a $d$-equidimensional complex manifold. Let $\mathscr{T}$ be a type for Green functions. Besides the properties (1)-(3) of Subsection 2.3, we assume the following additional property:

(4) For an open set $U$, if $u \in \mathscr{T}(U)$ and $v \in C^{\infty}(U)$, then $v u \in \mathscr{T}(U)$.

As examples, $C^{0}$ and $C^{\infty}$ have the property (4).

Lemma 2.4.1. Let $D$ be an $\mathbb{R}$-Cartier divisor on $X$. Let $\left\{U_{\lambda}\right\}$ be a locally finite covering of $X$ and let $\left\{\rho_{\lambda}\right\}_{\lambda \in \Lambda}$ be a partition of unity subordinate to the covering $\left\{U_{\lambda}\right\}_{\lambda \in \Lambda}$. Let $g_{\lambda}$ be a $\left(\left.D\right|_{U_{\lambda}}\right)$-Green function of $\mathscr{T}$-type on $U_{\lambda}$ for each $\lambda$. Then $g:=\sum_{\lambda} \rho_{\lambda} g_{\lambda}$ is a D-Green function of $\mathscr{T}$-type on $X$.

Proof. We set $D=a_{1} D_{1}+\cdots+a_{r} D_{r}$. Let $f_{i, x}$ be a local equation of $D_{i}$ on an open neighborhood $U_{x}$ of $x$. As $g_{\lambda}$ is a $\left(\left.D\right|_{U_{\lambda}}\right)$-Green function of $\mathscr{T}$-type on $U_{\lambda}$, for $\lambda$ with $x \in U_{\lambda}$,

$$
g_{\lambda}=v_{\lambda, x}-\sum a_{i} \log \left|f_{i, x}\right|^{2} \quad \text { (a.e.) }
$$


around $x$, where $v_{\lambda, x} \in \mathscr{T}\left(U_{\lambda} \cap U_{x}\right)$. Thus

$$
\begin{aligned}
g & =\sum_{\lambda} \rho_{\lambda}\left(v_{\lambda, x}-\sum a_{i} \log \left|f_{i, x}\right|^{2}\right) \quad \text { (a.e.) } \\
& =\left(\sum_{\lambda} \rho_{\lambda} v_{\lambda, x}\right)-\sum a_{i} \log \left|f_{i, x}\right|^{2}
\end{aligned}
$$

around $x$, as required.

The main result of this subsection is the following proposition.

Proposition 2.4.2. Let $g$ be a D-Green function of $\mathscr{T}$-type on $X$ and let

$$
D=b_{1} E_{1}+\cdots+b_{r} E_{r}
$$

be a decomposition such that $E_{1}, \ldots, E_{r} \in \operatorname{Div}(X)$ and $b_{1}, \ldots, b_{r} \in \mathbb{R}$. Note that $E_{i}$ is not necessarily a prime divisor. Then we have the following:

(1) There are locally integrable functions $g_{1}, \ldots, g_{r}$ such that $g_{i}$ is an $E_{i}$-Green function of $\mathscr{T}$-type for each $i$ and $g=b_{1} g_{1}+\cdots+b_{r} g_{r}$ (a.e.).

(2) If $E_{1}, \ldots, E_{r}$ are effective, $b_{1}, \ldots, b_{r} \in \mathbb{R}_{\geq 0}, g \geq 0$ (a.e.) and $g$ is of lower bounded type, then there are locally integrable functions $g_{1}, \ldots, g_{r}$ such that $g_{i}$ is a non-negative $E_{i}$-Green function of $\mathscr{T}$-type for each $i$ and $g=b_{1} g_{1}+\cdots+$ $b_{r} g_{r}$ (a.e.).

Proof. (1) Clearly we may assume that $b_{i} \neq 0$ for all $i$. Let $g_{i}^{\prime}$ be an $E_{i}$-Green function of $C^{\infty}$-type. Then there is $f \in \mathscr{T}(X)$ such that $f=g-\left(b_{1} g_{1}^{\prime}+\cdots+\right.$ $\left.b_{r} g_{r}^{\prime}\right)$ (a.e.). Thus

$$
g=b_{1}\left(g_{1}^{\prime}+f / b_{1}\right)+b_{2} g_{2}^{\prime}+\cdots+b_{r} g_{r}^{\prime} \quad \text { (a.e.) }
$$

(2) Clearly we may assume that $b_{i}>0$ for all $i$. First let us prove

Claim 2.4.2.1. For each $x \in X$, there are locally integrable functions $g_{1, x}, \ldots, g_{r, x}$ and an open neighborhood $U_{x}$ of $x$ such that $g_{i, x}$ is a non-negative $E_{i}$-Green function of $\mathscr{T}$-type on $U_{x}$ for every $i$, and $g=b_{1} g_{1, x}+\cdots+b_{r} g_{r, x}$ (a.e.) on $U_{x}$.

Proof. Let $U_{x}$ be a sufficiently small open neighborhood of $x$ and let $f_{i, x}$ be a local equation of $E_{i}$ on $U_{x}$ for every $i$. Let $g=v_{x}+\sum_{i=1}^{r}\left(-b_{i}\right) \log \left|f_{i, x}\right|^{2}$ (a.e.) be the local expression of $g$ on $U_{x}$ with respect to $f_{1, x}, \ldots, f_{r, x}$. We set $I=\left\{i \mid f_{i, x}(x)=0\right\}$ and $J=\left\{i \mid f_{i, x}(x) \neq 0\right\}$.

First we assume $I=\emptyset$. Then, shrinking $U_{x}$ if necessary, we may assume that

$$
v_{x}+\sum_{i=1}^{r}\left(-b_{i}\right) \log \left|f_{i, x}\right|^{2} \in \mathscr{T}\left(U_{x}\right)
$$


and $E_{i}=0$ on $U_{x}$ for all $i$. Thus if we set

$$
g_{i, x}=\left(1 / r b_{i}\right)\left(v_{x}+\sum_{i=1}^{r}\left(-b_{i}\right) \log \left|f_{i, x}\right|^{2}\right)
$$

for each $i$, then we have our assertion.

Next we consider the case where $I \neq \emptyset$. We put $f=v_{x}+\sum_{j \in J}\left(-b_{j}\right) \log \left|f_{j, x}\right|^{2}$. Then, shrinking $U_{x}$ if necessary, we may assume that $f \in \mathscr{T}\left(U_{x}\right)$ and is bounded below. We set

$$
g_{i, x}= \begin{cases}f /\left(b_{i} \#(I)\right)-\log \left|f_{i, x}\right|^{2} & \text { if } i \in I, \\ 0 & \text { if } i \in J .\end{cases}
$$

Note that $g=\sum_{i=1}^{r} b_{i} g_{i, x}$ (a.e.) and $g_{i, x} \geq 0$ around $x$ for $i \in I$. Thus, shrinking $U_{x}$ if necessary, we obtain our assertion.

By using the above claim, we can construct an open covering $\left\{U_{\lambda}\right\}_{\lambda \in \Lambda}$ and locally integrable functions $g_{1, \lambda}, \ldots, g_{r, \lambda}$ on $U_{\lambda}$ with the following properties:

(i) $\left\{U_{\lambda}\right\}_{\lambda \in \Lambda}$ is locally finite and the closure of $U_{\lambda}$ is compact for every $\lambda$.

(ii) $g_{i, \lambda}$ is a non-negative $E_{i}$-Green function of $\mathscr{T}$-type on $U_{\lambda}$ for every $i$.

(iii) $g=b_{1} g_{1, \lambda}+\cdots+b_{r} g_{r, \lambda}$ (a.e.) on $U_{\lambda}$.

Let $\left\{\rho_{\lambda}\right\}_{\lambda \in \Lambda}$ be a partition of unity subordinate to the covering $\left\{U_{\lambda}\right\}_{\lambda \in \Lambda}$. We set $g_{i}=\sum_{\lambda} \rho_{\lambda} g_{i, \lambda}$. Clearly $g_{i} \geq 0$ and

$$
g=\left.\sum_{\lambda} \rho_{\lambda} g\right|_{U_{\lambda}} \stackrel{\text { (a.e.) }}{=} \sum_{\lambda} \rho_{\lambda} \sum_{i=1}^{r} b_{i} g_{i, \lambda}=\sum_{i=1}^{r} b_{i} g_{i} .
$$

Moreover, by Lemma 2.4.1, $g_{i}$ is an $E_{i}$-Green function of $\mathscr{T}$-type.

\section{§2.5. Norms arising from Green functions}

Let $X$ be a $d$-equidimensional complex manifold. Let $g$ be a locally integral function on $X$. For $\phi \in \mathcal{M}(X)$, we define

$$
|\phi|_{g}:=\exp (-g / 2)|\phi|
$$

Moreover, the essential supremum of $|\phi|_{g}$ is denoted by $\|\phi\|_{g}$, that is,

$$
\|\phi\|_{g}:=\operatorname{ess} \sup \left\{|\phi|_{g}(x) \mid x \in X\right\} \text {. }
$$

Lemma 2.5.1. (1) $\|\cdot\|_{g}$ has the following properties:

(1.1) $\|\lambda \phi\|_{g}=|\lambda|\|\phi\|_{g}$ for all $\lambda \in \mathbb{C}$ and $\phi \in \mathcal{M}(X)$.

(1.2) $\|\phi+\psi\|_{g} \leq\|\phi\|_{g}+\|\psi\|_{g}$ for all $\phi, \psi \in \mathcal{M}(X)$.

(1.3) For $\phi \in \mathcal{M}(X),\|\phi\|_{g}=0$ if and only if $\phi=0$. 
(2) Let $V$ be a vector subspace of $\mathcal{M}(X)$ over $\mathbb{C}$. If $\|\phi\|_{g}<\infty$ for all $\phi \in V$, then $\|\cdot\|_{g}$ yields a norm on $V$. In particular, if $D$ is an $\mathbb{R}$-Cartier divisor, $g$ is a D-Green function of $\mathscr{T}$-type and $g$ is of lower bounded type, then $\|\cdot\|_{g}$ is a norm of $H_{\mathcal{M}}^{0}(X, D)$ (cf. Proposition 2.3.6), where $\mathscr{T}$ is a type for Green functions.

Proof. (1) (1.1) and (1.2) are obvious. If $\|\phi\|_{g}=0$, then $|\phi|_{g}=0$ (a.e.). Moreover, as $g$ is integrable, the measure of $\{x \in X \mid g(x)=\infty\}$ is zero. Thus $|\phi|=0$ (a.e.), and hence $\phi=0$.

(2) follows from (1).

Let $\Phi$ be a continuous volume form on $X$. For $\phi, \psi \in \mathcal{M}(X)$, if $\phi \bar{\psi} \exp (-g)$ is integrable, then we denote its integral

$$
\int_{X} \phi \bar{\psi} \exp (-g) \Phi
$$

by $\langle\phi, \psi\rangle_{g}$.

We assume that $g$ is a $D$-Green function of $C^{0}$-type. We set

$$
D=a_{1} D_{1}+\cdots+a_{l} D_{l},
$$

where $D_{i}$ 's are reduced and irreducible divisors on $X$ and $a_{1}, \ldots, a_{r} \in \mathbb{R}$. Let us fix $x \in X$. Let $f_{1}, \ldots, f_{l}$ be local equations of $D_{1}, \ldots, D_{l}$ around $x$, and let

$$
g=u+\sum_{i=1}^{l}\left(-a_{i}\right) \log \left|f_{i}\right|^{2} \quad \text { (a.e.) }
$$

be the local expression of $g$ around $x$ with respect to $f_{1}, \ldots, f_{l}$. For $\phi \in H_{\mathcal{M}}^{0}(X, D)$, we set $\phi=f_{1}^{b_{1}} \cdots f_{l}^{b_{l}} v$ around $x$, where $v$ has no factors of $f_{1}, \ldots, f_{l}$. Note that $b_{1}, \ldots, b_{l}$ do not depend on the choice of $f_{1}, \ldots, f_{l}$. Since $(\phi)+D \geq 0$, we have $a_{i}+b_{i} \geq 0$ for all $i$ and $v$ is holomorphic around $x$. Then

$$
|\phi|_{g}=\left|f_{1}\right|^{a_{1}+b_{1}} \cdots\left|f_{l}\right|^{a_{l}+b_{l}}|v| \exp (-u / 2) \quad \text { (a.e.). }
$$

Let us choose another set of local equations $f_{1}^{\prime}, \ldots, f_{l}^{\prime}$ of $D_{1}, \ldots, D_{l}$ around $x$, and let

$$
g=u^{\prime}+\sum_{i=1}^{l}\left(-a_{i}\right) \log \left|f_{i}^{\prime}\right|^{2} \quad \text { (a.e.) }
$$

be the local expression of $g$ around $x$ with respect to $f_{1}^{\prime}, \ldots, f_{l}^{\prime}$. Moreover, we set $\phi=f_{1}^{\prime b_{1}} \cdots f_{l}^{\prime b_{l}} v^{\prime}$ around $x$ as before. Then

$$
\left.|\phi|_{g}=\left|f_{1}^{\prime}\right|^{a_{1}+b_{1}} \ldots\left|f_{l}^{\prime}\right|^{a_{l}+b_{l}}\left|v^{\prime}\right| \exp \left(-u^{\prime} / 2\right) \quad \text { (a.e. }\right) \text {. }
$$


Note that

$$
\left|f_{1}\right|^{a_{1}+b_{1}} \cdots\left|f_{l}\right|^{a_{l}+b_{l}}|v| \exp (-u / 2) \quad \text { and } \quad\left|f_{1}^{\prime}\right|^{a_{1}+b_{1}} \cdots\left|f_{l}^{\prime}\right|^{a_{l}+b_{l}}\left|v^{\prime}\right| \exp \left(-u^{\prime} / 2\right)
$$

are continuous, so they are equal around $x$. This observation shows that there is a unique continuous function $h$ on $X$ such that $|\phi|_{g}=h$ (a.e.). Therefore, in the case where $g$ is of $C^{0}$-type, we always assume that $|\phi|_{g}$ means the above continuous function $h$. Then we have the following proposition.

Proposition 2.5.2. Let $g$ be a D-Green function of $C^{0}$-type.

(1) For $\phi \in H_{\mathcal{M}}^{0}(X, D),|\phi|_{g}$ is locally bounded above.

(2) If $X$ is compact, then $\langle\phi, \psi\rangle_{g}$ exists for $\phi, \psi \in H_{\mathcal{M}}^{0}(X, D)$. Moreover, $\langle,\rangle_{g}$ yields a hermitian inner product on $H_{\mathcal{M}}^{0}(X, D)$.

\section{$\S 3$. Gromov's inequality and distorsion functions for $\mathbb{R}$-Cartier divisors}

Let $X$ be a $d$-equidimensional compact complex manifold. Let $D$ be an $\mathbb{R}$-Cartier divisor on $X$ and let $g$ be a $D$-Green function of $C^{0}$-type. Let us fix a continuous volume form $\Phi$ on $X$. Recall that $|\phi|_{g},\|\phi\|_{g}$ and $\langle\phi, \psi\rangle_{g}$ for $\phi, \psi \in H_{\mathcal{M}}^{0}(X, D)$ are given by

$$
\begin{gathered}
|\phi|_{g}:=|\phi| \exp (-g / 2), \quad\|\phi\|_{g}:=\operatorname{ess} \sup \left\{|\phi|_{g}(x) \mid x \in X\right\}, \\
\langle\phi, \psi\rangle_{g}:=\int_{X} \phi \bar{\psi} \exp (-g) \Phi .
\end{gathered}
$$

As described in Subsection 2.5, we can view $|\phi|_{g}$ as a continuous function, so that $|\phi|_{g}$ is always assumed to be continuous.

In this section, we consider Gromov's inequality and distorsion functions for $\mathbb{R}$-Cartier divisors.

\section{§3.1. Gromov's inequality for $\mathbb{R}$-Cartier divisors}

Proposition 3.1.1 (Gromov's inequality for $\mathbb{R}$-Cartier divisors). Let $D_{1}, \ldots, D_{l}$ be $\mathbb{R}$-Cartier divisors on $X$ and let $g_{1}, \ldots, g_{l}$ be locally integrable functions on $X$ such that $g_{i}$ is a $D_{i}$-Green function of $C^{\infty}$-type for each $i$. Then there is a positive constant $C$ such that

$$
\|\phi\|_{a_{1} g_{1}+\cdots+a_{l} g_{l}}^{2} \leq C\left(1+\left|a_{1}\right|+\cdots+\left|a_{l}\right|\right)^{2 d}\langle\phi, \phi\rangle_{a_{1} g_{1}+\cdots+a_{l} g_{l}}
$$

for all $\phi \in H_{\mathcal{M}}^{0}\left(X, a_{1} D_{1}+\cdots+a_{l} D_{l}\right)$ and $a_{1}, \ldots, a_{l} \in \mathbb{R}$.

Proof. We can find distinct prime divisors $\Gamma_{1}, \ldots, \Gamma_{r}$ on $X$, locally integrable functions $\gamma_{1}, \ldots, \gamma_{r}$ on $X, C^{\infty}$-functions $f_{1}, \ldots, f_{l}$ and real numbers $\alpha_{i j}$ such that 
$\gamma_{j}$ is a $\Gamma_{j}$-Green function of $C^{\infty}$-type for each $j=1, \ldots, r$,

$$
D_{i}=\sum_{j=1}^{r} \alpha_{i j} \Gamma_{j} \quad \text { and } \quad g_{i}=f_{i}+\sum_{j=1}^{r} \alpha_{i j} \gamma_{j} \text { (a.e.). }
$$

Then

$$
\begin{aligned}
a_{1} D_{1}+\cdots+a_{l} D_{l} & =\sum_{j=1}^{r}\left(\sum_{i=1}^{l} a_{i} \alpha_{i j}\right) \Gamma_{j}+\sum_{i=1}^{l} a_{i} \quad \text { (the zero divisor) } \\
a_{1} g_{1}+\cdots+a_{l} g_{l} & =\sum_{j=1}^{r}\left(\sum_{i=1}^{l} a_{i} \alpha_{i j}\right) \gamma_{j}+\sum_{i=1}^{l} a_{i} f_{i} \quad \text { (a.e.). }
\end{aligned}
$$

Moreover, if we set $A=\max \left\{\left|\alpha_{i j}\right|\right\}$, then

$$
1+\sum_{i=1}^{l}\left|a_{i}\right|+\sum_{j=1}^{r}\left|\sum_{i=1}^{l} a_{i} \alpha_{i j}\right| \leq 1+(A r+1) \sum_{i=1}^{l}\left|a_{i}\right| \leq(A r+1)\left(1+\sum_{i=1}^{l}\left|a_{i}\right|\right) .
$$

Thus we may assume that $D_{1}, \ldots, D_{r}$ are distinct prime divisors and

$$
D_{r+1}=\cdots=D_{l}=0 .
$$

Let $U$ be an open set of $X$ over which there are local equations $f_{1}, \ldots, f_{r}$ of $D_{1}, \ldots, D_{r}$ respectively.

Claim 3.1.1.1. For all $\phi \in H_{\mathcal{M}}^{0}\left(X, a_{1} D_{1}+\cdots+a_{l} D_{l}\right)$ and $a_{1}, \ldots, a_{l} \in \mathbb{R}$, the product

$$
\phi f_{1}^{\left\lfloor a_{1}\right\rfloor} \cdots f_{r}^{\left\lfloor a_{r}\right\rfloor}
$$

is holomorphic over $U$, that is, there are $b_{1}, \ldots, b_{r} \in \mathbb{Z}$ and a holomorphic function $f$ on $U$ such that $\phi=f_{1}^{b_{1}} \cdots f_{r}^{b_{r}} f$ and $b_{1}+a_{1} \geq 0, \ldots, b_{r}+a_{r} \geq 0$.

Proof. Fix $x \in U$. Let $f_{i}=e_{i} f_{i 1} \cdots f_{i c_{i}}$ be the prime decomposition of $f_{i}$ in $\mathscr{O}_{X, x}$, where $e_{i} \in \mathscr{O}_{X, x}^{\times}$and $f_{i j}$ 's are distinct prime elements of $\mathscr{O}_{X, x}$. Let $D_{i j}$ be the prime divisor given by $f_{i j}$ around $x$. Since $\phi \in H_{\mathcal{M}}^{0}\left(X, a_{1} D_{1}+\cdots+a_{l} D_{l}\right)$, we have

$$
\begin{aligned}
& (\phi)+a_{1} D_{1}+\cdots+a_{l} D_{l} \\
& \quad=(\phi)+a_{1} D_{11}+\cdots+a_{1} D_{1 c_{1}}+\cdots+a_{r} D_{r 1}+\cdots+a_{r} D_{r c_{r}} \geq 0
\end{aligned}
$$

around $x$. Note that $D_{11}, \ldots, D_{1 c_{1}}, \ldots, D_{r 1}, \ldots, D_{r c_{r}}$ are distinct prime divisors around $x$. Thus $\phi f_{11}^{\left\lfloor a_{1}\right\rfloor} \cdots f_{1 c_{1}}^{\left\lfloor a_{1}\right\rfloor} \cdots f_{r 1}^{\left\lfloor a_{r}\right\rfloor} \cdots f_{r c_{r}}^{\left\lfloor a_{r}\right\rfloor}$ is holomorphic around $x$. Therefore, as

$$
f_{1}^{\left\lfloor a_{1}\right\rfloor} \cdots f_{r}^{\left\lfloor a_{r}\right\rfloor}=e_{1}^{\left\lfloor a_{1}\right\rfloor} \cdots e_{r}^{\left\lfloor a_{r}\right\rfloor} f_{11}^{\left\lfloor a_{1}\right\rfloor} \cdots f_{1 c_{1}}^{\left\lfloor a_{1}\right\rfloor} \cdots f_{r 1}^{\left\lfloor a_{r}\right\rfloor} \cdots f_{r c_{r}}^{\left\lfloor a_{r}\right\rfloor},
$$

$\phi f_{1}^{\left\lfloor a_{1}\right\rfloor} \cdots f_{r}^{\left\lfloor a_{r}\right\rfloor}$ is holomorphic around $x$. 
By the above observation, the assertion of the proposition follows from the local version below.

Lemma 3.1.2. Let $a, b, c$ be real numbers with $a>b>c>0$. Set

$$
U=\left\{z \in \mathbb{C}^{d}|| z \mid<a\right\}, \quad V=\left\{z \in \mathbb{C}^{d}|| z \mid<b\right\}, \quad W=\left\{z \in \mathbb{C}^{d}|| z \mid<c\right\} .
$$

Let $\Phi$ be a continuous volume form on $U, f_{1}, \ldots, f_{l} \in \mathscr{O}_{U}(U), v_{1}, \ldots, v_{l} \in C^{\infty}(U)$ and

$$
g_{i}=v_{i}-\log \left|f_{i}\right|^{2}
$$

for $i=1, \ldots, l$. For $a_{1}, \ldots, a_{l} \in \mathbb{R}$, set

$$
L\left(a_{1}, \ldots, a_{l}\right)=\left\{\begin{array}{l|l}
f_{1}^{b_{1}} \cdots f_{l}^{b_{l}} f & \begin{array}{l}
f \in \mathscr{O}_{U}(U) \text { and } b_{1}, \ldots, b_{l} \in \mathbb{Z} \text { with } \\
b_{1}+a_{1} \geq 0, \ldots, b_{l}+a_{l} \geq 0
\end{array}
\end{array}\right\} .
$$

(Note that $L\left(a_{1}, \ldots, a_{l}\right)$ is a complex vector space.) Then there is a positive constant $C$ such that

$$
\begin{aligned}
& \max _{z \in \bar{W}}\left\{|\phi|^{2} \exp \left(-a_{1} g_{1}-\cdots-a_{l} g_{l}\right)(z)\right\} \\
& \leq C\left(\left|a_{1}\right|+\cdots+\left|a_{l}\right|+1\right)^{2 d} \int_{V}|\phi|^{2} \exp \left(-a_{1} g_{1}-\cdots-a_{l} g_{l}\right) \Phi
\end{aligned}
$$

for all $\phi \in L\left(a_{1}, \ldots, a_{l}\right)$ and all $a_{1}, \ldots, a_{l} \in \mathbb{R}$.

Proof. We set

$$
u_{1}=\exp \left(-v_{1}\right), \ldots, u_{l}=\exp \left(-v_{l}\right), \quad u_{l+1}=\exp \left(v_{1}\right), \ldots, u_{2 l}=\exp \left(v_{l}\right) .
$$

Then in the same way as in [14, Lemma 1.1.1], we can find a positive constant $D$ with the following properties:

(a) For $x_{0}, x \in \bar{V}, u_{i}(x) \geq u_{i}\left(x_{0}\right)\left(1-D\left|x-x_{0}\right|^{\prime}\right)$ for all $i=1, \ldots, 2 l$, where $|z|^{\prime}=\left|z_{1}\right|+\cdots+\left|z_{d}\right|$ for $z=\left(z_{1}, \ldots, z_{d}\right) \in \mathbb{C}^{d}$.

(b) If $x_{0} \in \bar{W}$, then $B\left(x_{0}, 1 / D\right) \subseteq \bar{V}$, where

$$
B\left(x_{0}, 1 / D\right)=\left\{x \in \mathbb{C}^{d}|| x-\left.x_{0}\right|^{\prime} \leq 1 / D\right\} .
$$

We set

$$
\Phi_{\text {can }}=\left(\frac{\sqrt{-1}}{2}\right)^{d} d z_{1} \wedge d \bar{z}_{1} \wedge \cdots \wedge d z_{d} \wedge d \bar{z}_{d} .
$$

Then we can choose a positive constant $e$ with $\Phi \geq e \Phi_{\text {can }}$. For

$$
\phi=f_{1}^{b_{1}} \ldots f_{l}^{b_{l}} f \in L\left(a_{1}, \ldots, a_{l}\right),
$$


we assume that the continuous function

$$
\begin{aligned}
|\phi|^{2} \exp \left(-a_{1} g_{1}-\right. & \left.\cdots-a_{l} g_{l}\right) \\
& =\left|f_{1}\right|^{2\left(b_{1}+a_{1}\right)} \cdots\left|f_{l}\right|^{2\left(b_{l}+a_{l}\right)}|f|^{2} \exp \left(-a_{1} v_{1}-\cdots-a_{l} v_{l}\right)
\end{aligned}
$$

on $\bar{W}$ takes the maximal value at $x_{0} \in \bar{W}$. Let us choose $\epsilon_{i} \in\{ \pm 1\}$ such that $a_{i}=\epsilon_{i}\left|a_{i}\right|$. Note that

$$
\begin{aligned}
\exp \left(-a_{1} v_{1}(x)-\cdots-a_{l} v_{l}(x)\right)=\prod_{i=1}^{l} \exp \left(-\epsilon_{i} v_{i}(x)\right)^{\left|a_{i}\right|} \\
\geq\left(\prod_{i=1}^{l} \exp \left(-\epsilon_{i} v_{i}\left(x_{0}\right)\right)^{\left|a_{i}\right|}\right)\left(1-D\left|x-x_{0}\right|^{\prime}\right)^{\left|a_{1}\right|+\cdots+\left|a_{l}\right|} \\
=\exp \left(-a_{1} v_{1}\left(x_{0}\right)-\cdots-a_{l} v_{l}\left(x_{0}\right)\right)\left(1-D\left|x-x_{0}\right|^{\prime}\right)^{\left|a_{1}\right|+\cdots+\left|a_{l}\right|}
\end{aligned}
$$

on $B\left(x_{0}, 1 / D\right)$. Therefore,

$$
\begin{aligned}
\int_{V}|\phi|^{2} & \exp \left(-a_{1} g_{1}-\cdots-a_{l} g_{l}\right) \Phi \geq e \exp \left(-a_{1} v_{1}\left(x_{0}\right)-\cdots-a_{l} v_{l}\left(x_{0}\right)\right) \\
& \times \int_{B\left(x_{0}, 1 / D\right)}\left|f_{1}\right|^{2\left(b_{1}+a_{1}\right)} \cdots\left|f_{l}\right|^{2\left(b_{l}+a_{l}\right)}|f|^{2}\left(1-D\left|x-x_{0}\right|^{\prime}\right)^{\left|a_{1}\right|+\cdots+\left|a_{l}\right|} \Phi_{\text {can }} .
\end{aligned}
$$

If we set $x-x_{0}=\left(r_{1} \exp \left(\sqrt{-1} \theta_{1}\right), \ldots, r_{d} \exp \left(\sqrt{-1} \theta_{d}\right)\right)$, then, by using [8, Theorem 4.1.3] and the pluriharmonicity of $\left|f_{1}\right|^{2\left(b_{1}+a_{1}\right)} \cdots\left|f_{l}\right|^{2\left(b_{l}+a_{l}\right)}|f|^{2}$,

$$
\begin{aligned}
\int_{B\left(x_{0}, 1 / D\right)} \mid & \left.f_{1}\right|^{2\left(b_{1}+a_{1}\right)} \cdots\left|f_{l}\right|^{2\left(b_{l}+a_{l}\right)}|f|^{2}\left(1-D\left|x-x_{0}\right|^{\prime}\right)^{\left|a_{1}\right|+\cdots+\left|a_{l}\right|} \Phi_{\text {can }} \\
= & \int_{\substack{r_{1}+\cdots+r_{d} \leq 1 / D \\
r_{1} \geq 0, \ldots, r_{d} \geq 0}}\left(\int_{0}^{2 \pi} \cdots \int_{0}^{2 \pi}\left|f_{1}\right|^{2\left(b_{1}+a_{1}\right)} \cdots\left|f_{l}\right|^{2\left(b_{l}+a_{l}\right)}|f|^{2} d \theta_{1} \cdots d \theta_{d}\right) \\
& \times r_{1} \cdots r_{d}\left(1-D\left(r_{1}+\cdots+r_{d}\right)\right)^{\left|a_{1}\right|+\cdots+\left|a_{l}\right|} d r_{1} \cdots d r_{d} \\
\geq & (2 \pi)^{d}\left|f_{1}\left(x_{0}\right)\right|^{2\left(b_{1}+a_{1}\right)} \cdots\left|f_{l}\left(x_{0}\right)\right|^{2\left(b_{l}+a_{l}\right)}\left|f\left(x_{0}\right)\right|^{2} \\
& \times \int_{[0,1 /(d D)]^{d}} r_{1} \cdots r_{d}\left(1-D\left(r_{1}+\cdots+r_{d}\right)\right)^{\left|a_{1}\right|+\cdots+\left|a_{l}\right|} d r_{1} \cdots d r_{d} .
\end{aligned}
$$

Therefore,

$$
\begin{aligned}
\int_{V}|\phi|^{2} \exp ( & \left.-a_{1} g_{1}-\cdots-a_{l} g_{l}\right) \Phi \\
\geq & \frac{e(2 \pi)^{d}}{(d D)^{2 d}} \max _{z \in W}\left\{|\phi|^{2} \exp \left(-a_{1} g_{1}-\cdots-a_{l} g_{l}\right)(z)\right\} \\
& \times \int_{[0,1]^{d}} t_{1} \cdots t_{d}\left(1-(1 / d)\left(t_{1}+\cdots+t_{d}\right)\right)^{\left|a_{1}\right|+\cdots+\left|a_{l}\right|} d t_{1} \cdots d t_{d} .
\end{aligned}
$$

Hence our assertion follows from [14, Claim 1.1.1.1 in Lemma 1.1.1]. 


\section{§3.2. Distorsion functions for $\mathbb{R}$-Cartier divisors}

Let $D$ be an $\mathbb{R}$-Cartier divisor on $X$ and let $g$ be a $D$-Green function of $C^{0}$-type. Let $V$ be a complex vector subspace of $H_{\mathcal{M}}^{0}(X, D)$. Let $\phi_{1}, \ldots, \phi_{l}$ be an orthonormal basis of $V$ with respect to $\langle,\rangle_{g}$. It is easy to see that

$$
\left|\phi_{1}\right|_{g}^{2}+\cdots+\left|\phi_{l}\right|_{g}^{2}
$$

does not depend on the choice of the orthonormal basis $\phi_{1}, \ldots, \phi_{l}$ of $V$; it is denoted by $\operatorname{dist}(V ; g)$ and called the distorsion function of $V$ with respect to $g$.

Proposition 3.2.1. Let $V$ be a complex vector subspace of $H^{0}(X, D)$. Then

$$
|s|_{g}^{2}(x) \leq\langle s, s\rangle_{g} \operatorname{dist}(V ; g)(x) \quad(\forall x \in X)
$$

for all $s \in V$. In particular,

$$
|s|_{g}(x) \leq\left(\int_{X} \Phi\right)^{1 / 2}\|s\|_{g} \sqrt{\operatorname{dist}(V ; g)(x)} \quad(\forall x \in X) .
$$

Proof. Let $e_{1}, \ldots, e_{N}$ be an orthonormal basis of $V$ with respect to $\langle,\rangle_{g}$. If we set $s=a_{1} e_{1}+\cdots+a_{N} e_{N}$ for $s \in V$, then

$$
\langle s, s\rangle_{g}=\left|a_{1}\right|^{2}+\cdots+\left|a_{N}\right|^{2} .
$$

Therefore, by the Cauchy-Schwarz inequality,

$$
\begin{aligned}
|s|_{g}(x) & \leq\left|a_{1}\right|\left|e_{1}\right|_{g}(x)+\cdots+\left|a_{N}\right|\left|e_{N}\right|_{g}(x) \\
& \leq \sqrt{\left|a_{1}\right|^{2}+\cdots+\left|a_{N}\right|^{2}} \sqrt{\left|e_{1}\right|_{g}^{2}(x)+\cdots+\left|e_{N}\right|_{g}^{2}(x)} \\
& =\sqrt{\langle s, s\rangle_{g}} \sqrt{\operatorname{dist}(V ; g)(x)} .
\end{aligned}
$$

Lemma 3.2.2. Let $g^{\prime}$ be another D-Green function of $C^{0}$-type such that $g \leq$ $g^{\prime}$ (a.e.). Let $V$ be a complex vector subspace of $H^{0}(X, D)$. Then $\operatorname{dist}(V ; g) \leq$ $\exp \left(g^{\prime}-g\right) \operatorname{dist}\left(V ; g^{\prime}\right)$.

Proof. We can find a continuous function $u$ on $X$ such that $u \geq 0$ on $X$ and $g^{\prime}=g+u$ (a.e.). Let $\phi_{1}, \ldots, \phi_{l}$ be an orthonormal basis of $V$ with respect to $\langle,\rangle_{g^{\prime}}$ such that $\phi_{1}, \ldots, \phi_{l}$ are orthogonal with respect to $\langle,\rangle_{g}$. This is possible because any hermitian matrix is diagonalizable by a unitary matrix. Then

$$
\frac{\phi_{1}}{\sqrt{\left\langle\phi_{1}, \phi_{1}\right\rangle_{g}}}, \ldots, \frac{\phi_{l}}{\sqrt{\left\langle\phi_{l}, \phi_{l}\right\rangle_{g}}}
$$

form an orthonormal basis of $V$ with respect to $\langle,\rangle_{g}$. Thus

$$
\operatorname{dist}(V ; g)=\frac{\left|\phi_{1}\right|_{g}^{2}}{\left\langle\phi_{1}, \phi_{1}\right\rangle_{g}}+\cdots+\frac{\left|\phi_{l}\right|_{g}^{2}}{\left\langle\phi_{l}, \phi_{l}\right\rangle_{g}} .
$$


On the other hand, as $\left|\phi_{i}\right|_{g}^{2}=\left|\phi_{i}\right|_{g^{\prime}}^{2} \exp (u)$,

$$
\left\langle\phi_{i}, \phi_{i}\right\rangle_{g}=\int_{X}\left|\phi_{i}\right|_{g^{\prime}}^{2} \exp (u) \Phi \geq \int_{X}\left|\phi_{i}\right|_{g^{\prime}}^{2} \Phi=1
$$

Hence the lemma follows.

Let us consider the following fundamental estimate.

Theorem 3.2.3. Let $R=\bigoplus_{n>0} R_{n}$ be a graded subring of $\bigoplus_{n>0} H_{\mathcal{M}}^{0}(X, n D)$. If $g$ is a D-Green function of $C^{\infty}$-type, then there is a positive constant $C$ with the following properties:

(1) $\operatorname{dist}\left(R_{n} ; n g\right) \leq C(n+1)^{3 d}$ for all $n \geq 0$.

(2) $\frac{\operatorname{dist}\left(R_{n} ; n g\right)}{C(n+1)^{3 d}} \cdot \frac{\operatorname{dist}\left(R_{m} ; m g\right)}{C(m+1)^{3 d}} \leq \frac{\operatorname{dist}\left(R_{n+m} ;(n+m) g\right)}{C(n+m+1)^{3 d}}$ for all $n, m \geq 0$.

Proof. Let us begin with the following claim:

Claim 3.2.3.1. There is a positive constant $C_{1}$ such that $\operatorname{dist}\left(R_{n} ; n g\right) \leq$ $C_{1}(n+1)^{3 d}$ for all $n \geq 0$.

Proof. First of all, by Gromov's inequality for an $\mathbb{R}$-Cartier divisor (cf. Proposition 3.1.1), there is a positive constant $C^{\prime}$ such that

$$
\|\phi\|_{n g}^{2} \leq C^{\prime}(n+1)^{2 d}\langle\phi, \phi\rangle_{n g}
$$

for all $\phi \in H_{\mathcal{M}}^{0}(X, n D)$ and $n \geq 0$. Let $\phi_{1}, \ldots, \phi_{l_{n}}$ be an orthonormal basis of $R_{n}$. Then

$$
\begin{aligned}
& \operatorname{dist}\left(R_{n} ; n g\right) \leq\left\|\phi_{1}\right\|_{n g}^{2}+\cdots+\left\|\phi_{l_{n}}\right\|_{n g}^{2} \\
& \quad \leq C^{\prime}(n+1)^{2 d}\left(\left\langle\phi_{1}, \phi_{1}\right\rangle_{n g}+\cdots+\left\langle\phi_{l_{g}}, \phi_{l_{g}}\right\rangle_{n g}\right) \leq C^{\prime}(n+1)^{2 d} \operatorname{dim} R_{n},
\end{aligned}
$$

as required.

Claim 3.2.3.2. There is a positive constant $C_{2}$ such that

$$
\operatorname{dist}\left(R_{n} ; n g\right) \cdot \operatorname{dist}\left(R_{m} ; m g\right) \leq C_{2}(m+1)^{3 d} \operatorname{dist}\left(R_{n+m} ;(n+m) g\right)
$$

for $n \geq m \geq 0$.

Proof. Let $t_{1}, \ldots, t_{l}$ be an orthonormal basis of $R_{m}$. For each $j=1, \ldots, l$, we choose an orthonormal basis $s_{1}, \ldots, s_{r}$ of $R_{n}$ such that $s_{1} t_{j}, \ldots, s_{r} t_{j}$ are orthogonal in $R_{n+m}$. Note that the above $s_{1}, \ldots, s_{r}$ depend on $j$. We set $I=\{1 \leq i \leq r \mid$ $\left.s_{i} t_{j} \neq 0\right\}$. As

$$
\left\{\frac{s_{i} t_{j}}{\sqrt{\left\langle s_{i} t_{j}, s_{i} t_{j}\right\rangle_{(n+m) g}}}\right\}_{i \in I}
$$


can be extended to an orthonormal basis of $R_{n+m}$, we have

$$
\sum_{i \in I} \frac{\left|s_{i} t_{j}\right|_{(n+m) g}^{2}}{\left\langle s_{i} t_{j}, s_{i} t_{j}\right\rangle_{(n+m) g}} \leq \operatorname{dist}\left(R_{n+m} ;(n+m) g\right) .
$$

By using Gromov's inequality as in the previous claim, we obtain

$$
\left\langle s_{i} t_{j}, s_{i} t_{j}\right\rangle_{(n+m) g} \leq\left\langle s_{i}, s_{i}\right\rangle_{n g}\left\|t_{j}\right\|_{m g}^{2} \leq C^{\prime}(m+1)^{2 d}\left\langle t_{j}, t_{j}\right\rangle_{m g}=C^{\prime}(m+1)^{2 d} .
$$

Hence

$$
\begin{aligned}
\operatorname{dist}\left(R_{n} ; n g\right)\left|t_{j}\right|_{m g}^{2} & =\sum_{i=1}^{r}\left|s_{i} t_{j}\right|_{(n+m) g}=\sum_{i \in I}\left|s_{i} t_{j}\right|_{(n+m) g} \\
& \leq \sum_{i \in I} \frac{C^{\prime}(m+1)^{2 d}}{\left\langle s_{i} t_{j}, s_{i} t_{j}\right\rangle_{(n+m) g}}\left|s_{i} t_{j}\right|_{(n+m) g}^{2} \\
& \leq C^{\prime}(m+1)^{2 d} \operatorname{dist}\left(R_{n+m} ;(n+m) g\right),
\end{aligned}
$$

which implies

$$
\operatorname{dist}\left(R_{n} ; n g\right) \cdot \operatorname{dist}\left(R_{m} ; m g\right) \leq \operatorname{dim}\left(R_{m}\right) C^{\prime}(m+1)^{2 d} \operatorname{dist}\left(R_{n+m} ;(n+m) g\right),
$$

as required.

We set $C=\max \left\{C_{1}, 8^{d} C_{2}\right\}$. Then, for $n \geq m \geq 0$,

$$
\begin{aligned}
\frac{C(n+1)^{3 d} C(m+1)^{3 d}}{C(n+m+1)^{3 d}} & \geq C_{2}(m+1)^{3 d} 8^{d}\left(\frac{n+1}{n+m+1}\right)^{3 d} \\
& \geq C_{2}(m+1)^{3 d} 8^{d}\left(\frac{n+1}{2 n+1}\right)^{3 d} \\
& >C_{2}(m+1)^{3 d} 8^{d}\left(\frac{1}{2}\right)^{3 d}=C_{2}(m+1)^{3 d}
\end{aligned}
$$

Thus the proposition follows from the above claims.

\section{§4. Plurisubharmonic upper envelopes}

The main result of this section is the continuity of the upper envelope of a family of Green functions of $\mathrm{PSH}_{\mathbb{R}}$-type bounded above by a Green function of $C^{0}$-type. This will give the continuity of the positive part of the Zariski decomposition.

Throughout this section, let $X$ be a $d$-equidimensional complex manifold. Let us begin with the following fundamental estimate. 
Lemma 4.1. Let $f_{1}, \ldots, f_{r}$ be holomorphic functions on $X$ such that $f_{1}, \ldots, f_{r}$ are not zero on each connected component of $X$. Let $a_{1}, \ldots, a_{r} \in \mathbb{R}_{\geq 0}$ and $M \in \mathbb{R}$. Denote by $\operatorname{PSH}\left(X ; f_{1}, \ldots, f_{r}, a_{1}, \ldots, a_{r}, M\right)$ the set of all plurisubharmonic functions $u$ on $X$ such that

$$
u \leq M-\sum_{i=1}^{r} a_{i} \log \left|f_{i}\right|^{2} \quad \text { (a.e.) }
$$

over $X$. Then, for each point $x \in X$, there are an open neighborhood $U_{x}$ of $x$ and a constant $M_{x}^{\prime}$ depending only on $f_{1}, \ldots, f_{r}$ and $x$ such that

$$
u \leq M+M_{x}^{\prime}\left(a_{1}+\cdots+a_{r}\right)
$$

on $U_{x}$ for any $u \in \operatorname{PSH}\left(X ; f_{1}, \ldots, f_{r}, a_{1}, \ldots, a_{r}, M\right)$.

Proof. Let us begin with the following claim:

Claim 4.1.1. For any $u \in \operatorname{PSH}\left(X ; f_{1}, \ldots, f_{r}, a_{1}, \ldots, a_{r}, M\right)$,

$$
u \leq M-\sum_{i=1}^{r} a_{i} \log \left|f_{i}\right|^{2}
$$

over $X$.

Proof. Clearly we may assume that $a_{i}>0$ for all $i$. Let us fix $x \in X$. If $f_{i}(x)=0$ for some $i$, then the right hand side is $\infty$, so that the assertion is obvious. Now assume that $f_{i}(x) \neq 0$ for all $i$. Then the right hand side is continuous around $x$. Thus the assertion follows from Lemma 2.3.1.

Claim 4.1.2. Let $\epsilon \in \mathbb{R}_{>0}, a_{1}, \ldots, a_{d} \in \mathbb{R}_{\geq 0}$, and $M \in \mathbb{R}$. Then

$$
u \leq M-2 \log (\epsilon / 4)\left(a_{1}+\cdots+a_{d}\right)
$$

on $\Delta_{\epsilon / 4}^{d}$ for any $u \in \operatorname{PSH}\left(\Delta_{\epsilon}^{d} ; z_{1}, \ldots, z_{d}, a_{1}, \ldots, a_{d}, M\right)$, where $\left(z_{1}, \ldots, z_{d}\right)$ are the coordinates of $\mathbb{C}^{d}$ and

$$
\Delta_{t}^{d}=\left\{\left(z_{1}, \ldots, z_{d}\right) \in \mathbb{C}^{d}|| z_{1}|<t, \ldots,| z_{d} \mid<t\right\}
$$

for $t \in \mathbb{R}_{>0}$.

Proof. Note that if $\left(z_{1}, \ldots, z_{d}\right) \in \Delta_{\epsilon / 4}^{d}$, then

$$
\left\{\left(z_{1}+(\epsilon / 2) e^{2 \pi i \theta_{1}}, \ldots, z_{d}+(\epsilon / 2) e^{2 \pi i \theta_{d}}\right) \mid \theta_{1}, \ldots, \theta_{d} \in[0,1]\right\} \subseteq \Delta_{\epsilon}^{d} .
$$

Moreover, as

$$
\begin{aligned}
\epsilon / 2 & =\left|(\epsilon / 2) e^{2 \pi i \theta_{j}}\right|=\left|z_{j}+(\epsilon / 2) e^{2 \pi i \theta_{j}}-z_{j}\right| \\
& \leq\left|z_{j}+(\epsilon / 2) e^{2 \pi i \theta_{j}}\right|+\left|z_{j}\right|<\left|z_{j}+(\epsilon / 2) e^{2 \pi i \theta_{j}}\right|+\epsilon / 4,
\end{aligned}
$$


we have $\left|z_{j}+(\epsilon / 2) e^{2 \pi i \theta_{j}}\right|>\epsilon / 4$ for $j=1, \ldots, d$. Thus, by [8, Theorem 4.1.3],

$$
\begin{aligned}
u\left(z_{1}, \ldots, z_{d}\right) & \leq \int_{0}^{1} \cdots \int_{0}^{1} u\left(z_{1}+(\epsilon / 2) e^{2 \pi i \theta_{1}}, \ldots, z_{d}+(\epsilon / 2) e^{2 \pi i \theta_{d}}\right) d \theta_{1} \cdots d \theta_{d} \\
& \leq \int_{0}^{1} \cdots \int_{0}^{1}\left(M-\sum_{j=1}^{n} a_{j} \log \left|z_{j}+(\epsilon / 2) e^{2 \pi i \theta_{j}}\right|^{2}\right) d \theta_{1} \cdots d \theta_{d} \\
& =M-\sum_{j=1}^{d} a_{j} \int_{0}^{1} \log \left|z_{j}+(\epsilon / 2) e^{2 \pi i \theta_{j}}\right|^{2} d \theta_{j} \\
& \leq M-\sum_{j=1}^{d} a_{j} \int_{0}^{1} \log (\epsilon / 4)^{2} d \theta_{j}=M-2 \log (\epsilon / 4) \sum_{j=1}^{d} a_{j} .
\end{aligned}
$$

Next we observe the following:

Claim 4.1.3. If $\operatorname{Supp}\left\{x \in X \mid f_{1}(x) \cdots f_{r}(x)=0\right\}$ is a normal crossing divisor on $X$, then the assertion of the lemma holds.

Proof. We choose an open neighborhood $V_{x}$ such that $V_{x}=\Delta_{1}^{d}$ and

$$
\operatorname{Supp}\left\{x \in X \mid f_{1}(x) \cdots f_{r}(x)=0\right\}
$$

is given by $\left\{z_{1} \cdots z_{l}=0\right\}$. Then there are $b_{i j} \in \mathbb{Z}_{\geq 0}$ and nowhere vanishing holomorphic functions $v_{1}, \ldots, v_{r}$ on $\Delta_{1}^{d}$ such that

$$
f_{1}=z_{1}^{b_{11}} \ldots z_{l}^{b_{1 l}} v_{1}, \ldots, f_{r}=z_{1}^{b_{r 1}} \cdots z_{l}^{b_{r l}} v_{r} .
$$

Thus

$$
M-\sum_{i=1}^{r} a_{i} \log \left|f_{i}\right|^{2}=M-\sum_{i=1}^{r} a_{i} \log \left|v_{i}\right|^{2}-\sum_{j=1}^{l}\left(\sum_{i=1}^{r} a_{i} b_{i j}\right) \log \left|z_{j}\right|^{2} .
$$

We choose $M_{1}, M_{2} \in \mathbb{R}$ such that $M_{1}=\max \left\{b_{i j} \mid i=1, \ldots, r, j=1, \ldots, l\right\}$ and $M_{2} \geq \max _{z \in \Delta_{1 / 2}^{d}}\left\{-\log \left|v_{i}(z)\right|^{2}\right\}$ for all $i$. Then

$$
M-\sum_{i=1}^{r} a_{i} \log \left|f_{i}\right|^{2} \leq M+M_{2}\left(a_{1}+\cdots+a_{r}\right)-\sum_{j=1}^{l} M_{1}\left(a_{1}+\cdots+a_{r}\right) \log \left|z_{j}\right|^{2}
$$

on $\Delta_{1 / 2}^{d}$. Thus, by the previous claim, for any $u \in \operatorname{PSH}\left(X ; f_{1}, \ldots, f_{r}, a_{1}, \ldots, a_{r}, M\right)$,

$$
u \leq M+\left(M_{2}-2 \log (1 / 8) l M_{1}\right)\left(a_{1}+\cdots+a_{r}\right)
$$

on $\Delta_{1 / 8}^{d}$. 
Let us start considering the general case. Let $\pi: X^{\prime} \rightarrow X$ be a proper bimeromorphic map such that $\operatorname{Supp}\left(\left\{\pi^{*}\left(f_{1}\right) \cdots \pi^{*}\left(f_{r}\right)=0\right\}\right)$ is a normal crossing divisor on $X^{\prime}$. Note that if $u$ is a plurisubharmonic function on $X$, then $\pi^{*}(u)$ is also plurisubharmonic on $X^{\prime}$ (cf. [9, Corollary 2.9.5]). By the above claim, for each $y \in \pi^{-1}(x)$, there is an open neighborhood $U_{y}$ of $y$ and a constant $M_{y}^{\prime}$ depending only on $f_{1}, \ldots, f_{r}$ and $y$ such that, for any $u \in \operatorname{PSH}\left(X ; f_{1}, \ldots, f_{r}, a_{1}, \ldots, a_{r}, M\right)$,

$$
f^{*}(u) \leq M+M_{y}^{\prime}\left(a_{1}+\cdots+a_{r}\right)
$$

on $U_{y}$. As $\pi^{-1}(x) \subseteq \bigcup_{y \in \pi^{-1}(x)} U_{y}$ and $\pi^{-1}(x)$ is compact, there are $y_{1}, \ldots, y_{s}$ such that $\pi^{-1}(x) \subseteq U_{y_{1}} \cup \cdots \cup U_{y_{s}}$. We can choose an open neighborhood $U_{x}$ of $x$ such that $\pi^{-1}\left(U_{x}\right) \subseteq U_{y_{1}} \cup \cdots \cup U_{y_{s}}$. Thus, if we set $M_{x}^{\prime}=\max \left\{M_{y_{1}}^{\prime}, \ldots, M_{y_{s}}^{\prime}\right\}$, then

$$
f^{*}(u) \leq M+M_{x}^{\prime}\left(a_{1}+\cdots+a_{r}\right)
$$

on $\pi^{-1}\left(U_{x}\right)$, and hence the lemma follows.

Let $\alpha$ be a continuous $(1,1)$-form on $X$. We set

$$
\operatorname{PSH}(X ; \alpha):=\left\{\begin{array}{l|l}
\text { (i) } \phi: X \rightarrow\{-\infty\} \cup \mathbb{R} \\
\text { (ii) } \phi \in\left(C^{\infty}+\operatorname{PSH}\right)(X) \\
\text { (iii) }[\alpha]+d d^{c}([\phi]) \geq 0
\end{array}\right\} .
$$

First we observe the following lemma.

Lemma 4.2. Assume that $X$ is compact and that $\alpha+d d^{c}\left(\psi_{0}\right)$ is either positive or zero for some $C^{\infty}$-function $\psi_{0}$ on $X$. If $\phi \in \operatorname{PSH}(X ; \alpha) \cap C^{0}(X)$, then there are sequences $\left\{\phi_{n}\right\}_{n=1}^{\infty}$ and $\left\{\varphi_{n}\right\}_{n=1}^{\infty}$ in $\operatorname{PSH}(X ; \alpha) \cap C^{\infty}(X)$ such that $\phi_{n} \leq \phi \leq \varphi_{n}$ on $X$ for all $n \geq 1$ and

$$
\lim _{n \rightarrow \infty}\left\|\phi-\phi_{n}\right\|_{\text {sup }}=\lim _{n \rightarrow \infty}\left\|\varphi_{n}-\phi\right\|_{\text {sup }}=0 .
$$

Proof. First we assume that $\alpha=d d^{c}\left(-\psi_{0}\right)$ for some $C^{\infty}$-function $\psi_{0}$ on $X$. Then

$$
\operatorname{PSH}(X ; \alpha)=\left\{\psi_{0}+c \mid c \in \mathbb{R} \cup\{-\infty\}\right\}
$$

because $X$ is compact. Thus the assertion of the lemma is obvious.

Next we assume that $\alpha$ is positive. By [4, Theorem 1], there is a sequence of $\left\{\varphi_{n}\right\}_{n=1}^{\infty}$ in $\operatorname{PSH}(X ; \alpha) \cap C^{\infty}(X)$ such that

$$
\varphi_{1}(x) \geq \varphi_{2}(x) \geq \cdots \geq \phi(x)
$$

and $\phi(x)=\lim _{n \rightarrow \infty} \varphi_{n}(x)$ for all $x \in X$. Since $X$ is compact and $\phi$ is continuous, it is easy to see that $\lim _{n \rightarrow \infty}\left\|\varphi_{n}-\phi\right\|_{\text {sup }}=0$. We set $\phi_{n}=\varphi_{n}-\left\|\varphi_{n}-\phi\right\|_{\text {sup }}$ for 
all $n \geq 1$. Then $\phi_{n} \in \operatorname{PSH}(X ; \alpha) \cap C^{\infty}(X)$ and $\phi_{n} \leq \phi$. Note that $\left\|\phi-\phi_{n}\right\|_{\text {sup }} \leq$ $2\left\|\varphi_{n}-\phi\right\|_{\text {sup }}$. Thus $\lim _{n \rightarrow \infty}\left\|\phi-\phi_{n}\right\|_{\text {sup }}=0$.

Finally we assume that $\alpha^{\prime}=\alpha+d d^{c}\left(\psi_{0}\right)$ is positive for some $C^{\infty}$-function $\psi_{0}$ on $X$. Then

$$
\phi^{\prime}:=\phi-\psi_{0} \in \operatorname{PSH}\left(X ; \alpha^{\prime}\right) \cap C^{0}(X) .
$$

Thus, by the previous observation, there are sequences $\left\{\phi_{n}^{\prime}\right\}_{n=1}^{\infty}$ and $\left\{\varphi_{n}^{\prime}\right\}_{n=1}^{\infty}$ in $\operatorname{PSH}\left(X ; \alpha^{\prime}\right) \cap C^{\infty}(X)$ such that $\phi_{n}^{\prime} \leq \phi^{\prime} \leq \varphi_{n}^{\prime}$ on $X$ for all $n \geq 1$ and

$$
\lim _{n \rightarrow \infty}\left\|\phi^{\prime}-\phi_{n}^{\prime}\right\|_{\text {sup }}=\lim _{n \rightarrow \infty}\left\|\varphi_{n}^{\prime}-\phi^{\prime}\right\|_{\text {sup }}=0 .
$$

We set $\phi_{n}:=\phi_{n}^{\prime}+\psi_{0}$ and $\varphi_{n}:=\varphi_{n}^{\prime}+\psi_{0}$ for every $n \geq 1$. Then

$$
\phi_{n}, \varphi_{n} \in \operatorname{PSH}(X ; \alpha) \cap C^{\infty}(X) \text { and } \phi_{n} \leq \phi \leq \varphi_{n}
$$

for all $n \geq 1$. Moreover, $\lim _{n \rightarrow \infty}\left\|\phi_{n}-\phi\right\|_{\text {sup }}=\lim _{n \rightarrow \infty}\left\|\varphi_{n}-\phi\right\|_{\text {sup }}=0$.

Let $A$ be an $\mathbb{R}$-Cartier divisor and let $g_{A}$ be an $A$-Green function of $C^{\infty}$-type on $X$. Let $\alpha=c_{1}\left(A, g_{A}\right)$, that is, $\alpha$ is a $C^{\infty}$-form such that

$$
[\alpha]=d d^{c}\left(\left[g_{A}\right]\right)+\delta_{A}
$$

(cf. Proposition 2.3.2). Let us now consider the natural correspondence between $G_{\mathrm{PSH}}(X ; A)$ and $\operatorname{PSH}(X ; \alpha)$ in terms of $g_{A}$.

Proposition 4.3. If $\phi \in \operatorname{PSH}(X ; \alpha)$, then $\phi+g_{A} \in G_{\mathrm{PSH}}(X ; A)$. Moreover, we have the following:

(1) For $u \in G_{\mathrm{PSH}}(X ; A)$, there is $\phi \in \operatorname{PSH}(X ; \alpha)$ such that $\phi+g_{A}=u$ (a.e.).

(2) For $\phi_{1}, \phi_{2} \in \operatorname{PSH}(X ; \alpha)$,

$$
\phi_{1} \leq \phi_{2} \Leftrightarrow \phi_{1}+g_{A} \leq \phi_{2}+g_{A} \text { (a.e.). }
$$

(3) For $\phi \in \operatorname{PSH}(X ; \alpha)$,

$$
\phi(x) \neq-\infty(\forall x \in X) \Leftrightarrow \phi+g_{A} \in G_{\mathrm{PSH}_{\mathbb{R}}}(X ; A) .
$$

(4) For $\phi \in \operatorname{PSH}(X ; \alpha)$,

$$
\phi \in C^{\infty}(X) \Leftrightarrow \phi+g_{A} \in G_{C^{\infty}}(X ; A) .
$$

(5) For $\phi \in \operatorname{PSH}(X ; \alpha)$,

$$
\phi \in C^{0}(X) \Leftrightarrow \phi+g_{A} \in G_{C^{0}}(X ; A) .
$$


Proof. We set $A=a_{1} D_{1}+\cdots+a_{l} D_{l}$, where $D_{i}$ 's are reduced and irreducible divisors on $X$ and $a_{1}, \ldots, a_{l} \in \mathbb{R}$. Let $U$ be an open set of $X$ and let $f_{1}, \ldots, f_{l}$ be local equations of $D_{1}, \ldots, D_{l}$ on $U$ respectively. Let

$$
g_{A}=h-\sum_{i=1}^{l} a_{i} \log \left|f_{i}\right|^{2} \quad \text { (a.e.) }
$$

be the local expression of $g_{A}$ with respect to $f_{1}, \ldots, f_{l}$, where $h \in C^{\infty}(U)$. Then

$$
g_{A}+\phi=(h+\phi)-\sum_{i=1}^{l} a_{i} \log \left|f_{i}\right|^{2} \quad \text { (a.e.). }
$$

Since $\alpha=d d^{c}(h)$ on $U$, we have

$$
d d^{c}([h+\phi])=[\alpha]+d d^{c}([\phi]) \geq 0 .
$$

Thus $g_{A}+\phi \in G_{\mathrm{PSH}}(X ; A)$ and

$$
g_{A}+\phi=(h+\phi)-\sum_{i=1}^{l} a_{i} \log \left|f_{i}\right|^{2} \quad \text { (a.e.) }
$$

is the local expression of $g_{A}+\phi$ with respect to $f_{1}, \ldots, f_{l}$.

(1) For $u \in G_{\mathrm{PSH}}(X ; A)$, let

$$
u=p-\sum_{i=1}^{l} a_{i} \log \left|f_{i}\right|^{2} \quad \text { (a.e.) }
$$

be the local expression of $u$ with respect to $f_{1}, \ldots, f_{l}$, where $p$ is plurisubharmonic. It is easy to see that $p-h$ does not depend on the choice of the local equations $f_{1}, \ldots, f_{l}$. Thus there is a function $\phi: X \rightarrow\{-\infty\} \cup \mathbb{R}$ such that $\phi$ is locally given by $p-h$. Moreover

$$
d d^{c}([p-h])+[\alpha]=d d^{c}([p]) \geq 0 .
$$

Hence $\phi \in \operatorname{PSH}(X ; \alpha)$ and $\phi+g_{A}=u$ (a.e.).

(2) Clearly

$$
\phi_{1} \leq \phi_{2} \text { (a.e.) } \Leftrightarrow \phi_{1}+g_{A} \leq \phi_{2}+g_{A} \text { (a.e.). }
$$

On the other hand, by Lemma 2.3.1,

$$
\phi_{1} \leq \phi_{2} \Leftrightarrow \phi_{1} \leq \phi_{2} \text { (a.e.). }
$$

(3)-(5) are obvious because

$$
\phi+g_{A}=(h+\phi)-\sum_{i=1}^{l} a_{i} \log \left|f_{i}\right|^{2} \text { (a.e.) }
$$

is a local expression of $\phi+g_{A}$ and $h$ is $C^{\infty}$. 
Let $\mathscr{T}$ be a type for Green functions on $X$ such that PSH is a subjacent type of $\mathscr{T}$, that is, for an arbitrary open set $U$ of $X$, if $u \leq v$ (a.e.) on $U$ for $u \in \operatorname{PSH}(U)$ and $v \in \mathscr{T}(U)$, then $u \leq v$ on $U$.

Proposition 4.4. Let $A$ and $B$ be $\mathbb{R}$-Cartier divisors on $X$ with $A \leq B$, Let $h$ be a B-Green function of $\mathscr{T}$-type on $X$ such that $h$ is of upper bounded type. Let $\left\{g_{\lambda}\right\}_{\lambda \in \Lambda}$ be a family of A-Green functions of PSH-type on X. Assume that $g_{\lambda} \leq h$ (a.e.) for all $\lambda \in \Lambda$. Then there is an A-Green function $g$ of PSH-type on $X$ with the following properties:

(a) Fix an A-Green function $g_{A}$ of $C^{\infty}$-type. Let $\alpha$ be a unique $C^{\infty}$-form with $[\alpha]=d d^{c}\left(\left[g_{A}\right]\right)+\delta_{A}$. If we choose $\phi \in \operatorname{PSH}(X ; \alpha)$ and $\phi_{\lambda} \in \operatorname{PSH}(X ; \alpha)$ for each $\lambda \in \Lambda$ such that $g=g_{A}+\phi$ (a.e.) and $g_{\lambda}=g_{A}+\phi_{\lambda}$ (a.e.) (cf. Proposition 4.3), then $\phi$ is the upper semicontinuous regularization of the function given by

$$
x \mapsto \sup _{\lambda \in \Lambda} \phi_{\lambda}(x) .
$$

In particular, $g_{\text {can }}$ is the upper semicontinuous regularization of the function

$$
x \mapsto \sup _{\lambda \in \Lambda}\left(g_{\lambda}\right)_{\operatorname{can}}(x)
$$

over $X \backslash \operatorname{Supp}(A)$.

(b) $g \leq h$ (a.e.).

(c) If there is $g_{\lambda}$ such that $g_{\lambda} \in G_{\mathrm{PSH}_{\mathbb{R}}}(X ; A)$, then $g \in G_{\mathrm{PSH}_{\mathbb{R}}}(X ; A)$.

Proof. Let $A=a_{1} D_{1}+\cdots+a_{l} D_{l}$ and $B=b_{1} D_{1}+\cdots+b_{l} D_{l}$ be the decompositions of $A$ and $B$ such that $D_{i}$ 's are reduced and irreducible divisors, $a_{1}, \ldots, a_{l}, b_{1}, \ldots, b_{l}$ $\in \mathbb{R}$ and $D_{1} \cup \cdots \cup D_{l}=\operatorname{Supp}(A) \cup \operatorname{Supp}(B)$. Let $U$ be an open set of $X$ and let $f_{1}, \ldots, f_{l}$ be local equations of $D_{1}, \ldots, D_{l}$ over $U$ respectively. Let

$$
h=v+\sum_{i=1}^{l}\left(-b_{i}\right) \log \left|f_{i}\right|^{2} \quad \text { (a.e.) }
$$

be the local expression of $h$ with respect to $f_{1}, \ldots, f_{l}$. Moreover, let

$$
g_{\lambda}=u_{\lambda}+\sum_{i=1}^{l}\left(-a_{i}\right) \log \left|f_{i}\right|^{2} \quad \text { (a.e.) }
$$

be the local expression of $g_{\lambda}$ with respect to $f_{1}, \ldots, f_{l}$. Then

$$
u_{\lambda} \leq v-\sum_{i=1}^{l}\left(b_{i}-a_{i}\right) \log \left|f_{i}\right|^{2} \quad \text { (a.e.) }
$$


for every $\lambda \in \Lambda$. Note that $v$ is locally bounded above. Thus $\left\{u_{\lambda}\right\}_{\lambda \in \Lambda}$ is uniformly locally bounded above by Lemma 4.1. Let $u$ be the function on $U$ given by

$$
u(x)=\sup \left\{u_{\lambda}(x) \mid \lambda \in \Lambda\right\} .
$$

Let $\tilde{u}$ be the upper semicontinuous regularization of $u$. Then $\tilde{u}$ is plurisubharmonic on $U$ (cf. Subsection 2.1). Let $f_{1}^{\prime}, \ldots, f_{l}^{\prime}$ be another set of local equations of $D_{1}, \ldots, D_{l}$. Then there are $e_{1}, \ldots, e_{n} \in \mathscr{O}_{U}^{\times}(U)$ such that $f_{i}^{\prime}=e_{i} f_{i}$ for all $i$, so that

$$
g_{\lambda}=\left(u_{\lambda}+\sum_{i=1} a_{i} \log \left|e_{i}\right|^{2}\right)+\sum_{i=1}^{l}\left(-a_{i}\right) \log \left|f_{i}^{\prime}\right|^{2} \quad \text { (a.e.) }
$$

is the local expression of $g_{\lambda}$ with respect to $f_{1}^{\prime}, \ldots, f_{l}^{\prime}$. Thus, if we denote the plurisubharmonic function arising from $f_{1}^{\prime}, \ldots, f_{l}^{\prime}$ by $\tilde{u}^{\prime}$, then, by Lemma 2.3.1,

$$
\tilde{u}^{\prime}=\tilde{u}+\sum_{i=1}^{l} a_{i} \log \left|e_{i}\right|^{2}
$$

This means that

$$
\tilde{u}+\sum_{i=1}^{l}\left(-a_{i}\right) \log \left|f_{i}\right|^{2}
$$

does not depend on the choice of $f_{1}, \ldots, f_{l}$ over $U \backslash \operatorname{Supp}(A)$. Thus there is $g \in$ $G_{\mathrm{PSH}}(X ; A)$ such that

$$
\left.g\right|_{U}=\tilde{u}+\sum_{i=1}^{l}\left(-a_{i}\right) \log \left|f_{i}\right|^{2} \quad \text { (a.e.). }
$$

Let $g_{A}=u_{A}+\sum_{i=1}^{l}\left(-a_{i}\right) \log \left|f_{i}\right|^{2}$ (a.e.) be the local expression of $g_{A}$ with respect to $f_{1}, \ldots, f_{l}$. Then $\phi_{\lambda}=u_{\lambda}-u_{A}$ and $\phi=\tilde{u}-u_{A}$. Thus (a) follows.

By (a), $g_{\text {can }}$ is the upper semicontinuous regularization of the function $g^{\prime}$ given by $g^{\prime}(x)=\sup _{\lambda \in \Lambda}\left\{\left(g_{\lambda}\right)_{\text {can }}(x)\right\}$ over $X \backslash \operatorname{Supp}(A)$. As PSH is a subjacent type of $\mathscr{T}$, we have $\left(g_{\lambda}\right)_{\text {can }} \leq h_{\text {can }}$ on $X \backslash(\operatorname{Supp}(A) \cup \operatorname{Supp}(B))$ for all $\lambda \in \Lambda$. Note that $g=g^{\prime}$ (a.e.) (cf. Subsection 2.1). Thus we have $g \leq h$ (a.e.).

Finally we assume that $g_{\lambda} \in G_{\mathrm{PSH}_{\mathbb{R}}}(X ; A)$ for some $\lambda$. Then $u_{\lambda} \leq \tilde{u}$ (a.e.), so that $u_{\lambda} \leq \tilde{u}$ by Lemma 2.3.1. Thus $\tilde{u}(x) \neq-\infty$. Therefore, $g \in G_{\mathrm{PSH}_{\mathbb{R}}}(X ; A)$.

Let $A$ be an $\mathbb{R}$-Cartier divisor on $X$ and let $g$ be a locally integrable function on $X$. We set

$$
G_{\mathscr{T}}(X ; A)_{\leq g}:=\left\{u \in G_{\mathscr{T}}(X ; A) \mid u \leq g \text { (a.e.) }\right\},
$$

where $G_{\mathscr{T}}(X ; A)$ is the set of all $A$-Green functions of $\mathscr{T}$-type on $X$. 
Lemma 4.5. Let $A$ and $B$ be $\mathbb{R}$-Cartier divisors on $X$ with $A \leq B$. Let $g_{B}$ be $a$ $B$-Green function of $C^{\infty}$-type (resp. $C^{0}$-type). There is an A-Green function $g_{A}$ of $C^{\infty}$-type (resp. $C^{0}$-type) such that

$$
g_{A} \leq g_{B} \text { (a.e.) } \quad \text { and } \quad G_{\mathrm{PSH}}(X ; A)_{\leq g_{A}}=G_{\mathrm{PSH}}(X ; A)_{\leq g_{B}} .
$$

Proof. We set $A=a_{1} D_{1}+\cdots+a_{n} D_{n}$ and $B=b_{1} D_{1}+\cdots+b_{n} D_{n}$, where $D_{i}$ 's are reduced and irreducible divisors on $X$ and $a_{1}, \ldots, a_{n}, b_{1}, \ldots, b_{n} \in \mathbb{R}$. For $x \in X$, let $U_{x}$ be a small open neighborhood of $x$ and let $f_{1}, \ldots, f_{n}$ be local equations of $D_{1}, \ldots, D_{n}$ on $U_{x}$ respectively. Note that if $x \notin D_{i}$, then we take $f_{i}$ as the constant function 1. Let $g_{B}=h_{x}-\sum_{i} b_{i} \log \left|f_{i}\right|^{2}$ (a.e.) be the local expression of $g_{B}$ on $U_{x}$ with respect to $f_{1}, \ldots, f_{n}$. Shrinking $U_{x}$ if necessary, we may assume that there is a constant $M_{x}$ such that $\left|h_{x}\right| \leq M_{x}$ on $U_{x}$.

Claim 4.5.1. There are an open neighborhood $V_{x}$ of $x$ and a positive constant $C_{x}$ such that $V_{x} \subseteq U_{x}$,

$$
h_{x}+C_{x}-\sum_{i} a_{i} \log \left|f_{i}\right|^{2} \leq g_{B} \quad \text { (a.e.) }
$$

on $V_{x}$, and

on $V_{x}$ for all $u \in G_{\mathrm{PSH}}(X ; A)_{\leq g_{B}}$

$$
u \leq h_{x}+C_{x}-\sum_{i} a_{i} \log \left|f_{i}\right|^{2} \quad \text { (a.e.) }
$$

Proof. For $u \in G_{\mathrm{PSH}}(X ; A)_{\leq g_{B}}$, let $u=p_{x}(u)-\sum_{i} a_{i} \log \left|f_{i}\right|^{2}$ (a.e.) be the local expression of $u$ on $U_{x}$ with respect to $f_{1}, \ldots, f_{n}$. Then $u \leq g_{B}$ (a.e.) is nothing other than

$$
\left.p_{x}(u) \leq h_{x}-\sum_{i}\left(b_{i}-a_{i}\right) \log \left|f_{i}\right|^{2} \quad \text { (a.e. }\right)
$$

If either $a_{i}=b_{i}$ or $x \notin D_{i}$ for all $i$, then $\sum_{i}\left(b_{i}-a_{i}\right) \log \left|f_{i}\right|^{2}=0$ on $U_{x}$. Thus our assertion is obvious by taking $C_{x}=0$, so that we may assume that $a_{i}<b_{i}$ and $x \in D_{i}$ for some $i$. By Lemma 4.1, there are an open neighborhood $U_{x}^{\prime}$ of $x$ and a positive constant $M_{x}^{\prime}$ such that $U_{x}^{\prime} \subseteq U_{x}$ and $p_{x}(u) \leq M_{x}^{\prime}$ on $U_{x}^{\prime}$ for all $u \in G_{\mathrm{PSH}}(X ; A)_{\leq g_{B}}$. Note that

$$
M_{x}^{\prime}=-M_{x}+\left(M_{x}^{\prime}+M_{x}\right) \leq h_{x}+\left(M_{x}^{\prime}+M_{x}\right)
$$

on $U_{x}$. Thus if we set $C_{x}=M_{x}^{\prime}+M_{x}$, then $p_{x}(u) \leq h_{x}+C_{x}$ on $U_{x}^{\prime}$ for all $u \in G_{\mathrm{PSH}}(X ; A)_{\leq g_{B}}$. As $\lim _{y \rightarrow x} \sum_{i}\left(b_{i}-a_{i}\right) \log \left|f_{i}\right|^{2}(y)=-\infty$, we can find an open neighborhood $V_{x}$ of $x$ such that $V_{x} \subseteq U_{x}^{\prime}$ and $C_{x} \leq-\sum_{i}\left(b_{i}-a_{i}\right) \log \left|f_{i}\right|^{2}$ on $V_{x}$. Therefore,

$$
p_{x}(u) \leq h_{x}+C_{x} \leq h_{x}-\sum_{i}\left(b_{i}-a_{i}\right) \log \left|f_{i}\right|^{2}
$$

on $V_{x}$ for all $u \in G_{\mathrm{PSH}}(X ; A)_{\leq g_{B}}$, as required. 
By using Claim 4.5.1, we can find an open covering $\left\{V_{\lambda}\right\}_{\lambda \in \Lambda}$ of $X$ and a family $\left\{C_{\lambda}\right\}_{\lambda \in \Lambda}$ of constants with the following properties:

(1) $\left\{V_{\lambda}\right\}_{\lambda \in \Lambda}$ is a locally finite covering.

(2) There are local equations $f_{\lambda, 1}, \ldots, f_{\lambda, n}$ of $D_{1}, \ldots, D_{n}$ on $V_{\lambda}$ respectively.

(3) Let $g_{B}=h_{\lambda}-\sum_{i} b_{i} \log \left|f_{\lambda, i}\right|^{2}$ (a.e.) be the local expression of $g_{B}$ on $V_{\lambda}$ with respect to $f_{\lambda, 1}, \ldots, f_{\lambda, n}$. Then

$$
h_{\lambda}+C_{\lambda}-\sum a_{i} \log \left|f_{\lambda, i}\right|^{2} \leq g_{B} \quad \text { (a.e.) }
$$

on $V_{\lambda}$, and

$$
u \leq h_{\lambda}+C_{\lambda}-\sum_{i} a_{i} \log \left|f_{\lambda, i}\right|^{2} \quad \text { (a.e.) }
$$

on $V_{\lambda}$ for all $u \in G_{\mathrm{PSH}}(X ; A)_{\leq g_{B}}$.

Let $\left\{\rho_{\lambda}\right\}_{\lambda \in \Lambda}$ be a partition of unity subordinate to the covering $\left\{V_{\lambda}\right\}_{\lambda \in \Lambda}$. We set

$$
g_{A}=\sum_{\lambda} \rho_{\lambda}\left(h_{\lambda}+C_{\lambda}-\sum_{i} a_{i} \log \left|f_{\lambda, i}\right|^{2}\right) .
$$

By Lemma 2.4.1, $g_{A}$ is an $A$-Green function of $C^{\infty}$-type (resp. $C^{0}$-type). Moreover, $g_{A} \leq g_{B}$ (a.e.) and $u \leq g_{A}$ (a.e.) for all $u \in G_{\mathrm{PSH}}(X ; A)_{\leq g_{B}}$. Hence the lemma follows.

The following theorem is the main result of this section.

Theorem 4.6. Let $A$ be an $\mathbb{R}$-Cartier divisor on $X$. If $X$ is projective and there is an A-Green function $h$ of $C^{\infty}$-type such that $d d^{c}([h])+\delta_{A}$ is represented by either a positive $C^{\infty}$-form or the zero form, then we have the following:

(1) Let $B$ be an $\mathbb{R}$-Cartier divisor on $X$ with $A \leq B$. Let $g_{B}$ be a B-Green function of $C^{0}$-type. Then there is $g \in G_{C^{0} \cap \mathrm{PSH}}(X ; A)$ such that $g \leq g_{B}$ (a.e.) and

$$
u \leq g \text { (a.e. }) \quad\left(\forall u \in G_{\mathrm{PSH}}(X ; A)_{\leq g_{B}}\right) .
$$

(2) If $u \in G_{C^{0} \cap \mathrm{PSH}}(X ; A)$, then there are sequences $\left\{u_{n}\right\}_{n=1}^{\infty}$ and $\left\{v_{n}\right\}_{n=1}^{\infty}$ of continuous functions on $X$ with the following properties:

(2.1) $u_{n} \geq 0$ and $v_{n} \geq 0$ for all $n \geq 1$.

(2.2) $\lim _{n \rightarrow \infty}\left\|u_{n}\right\|_{\text {sup }}=\lim _{n \rightarrow \infty}\left\|v_{n}\right\|_{\text {sup }}=0$.

(2.3) $u-u_{n}, u+v_{n} \in G_{C^{\infty} \cap \mathrm{PSH}}(X ; A)$ all $n \geq 1$.

Proof. (1) Let us begin with the following claim: 
Claim 4.6.1. There is $g \in G_{\mathrm{PSH}_{\mathbb{R}}}(X ; A)$ such that $g \leq g_{B}$ (a.e.) and

$$
u \leq g \quad \text { (a.e. }) \quad\left(\forall u \in G_{\mathrm{PSH}}(X ; A)_{\leq g_{B}}\right) .
$$

We say $g$ is the greatest element of $G_{\mathrm{PSH}}(X ; A)_{\leq g_{B}}$ modulo null functions.

Proof. Note that PSH is a subjacent type of $C^{0}$ by Lemma 2.3.1, and that $h-c \in$ $G_{\mathrm{PSH}_{\mathbb{R}}}(X ; A)_{\leq g_{B}}$ for some constant $c$. Thus the assertion follows from Proposition 4.4.

Claim 4.6.2. If $g_{B}$ is of $C^{\infty}$-type, then the assertion of (1) holds.

Proof. By Lemma 4.5, we may assume that $A=B$. Let $\alpha=c_{1}\left(A, g_{A}\right)$, that is, $\alpha$ is a $C^{\infty}$-form such that $[\alpha]=d d^{c}\left(\left[g_{A}\right]\right)+\delta_{A}$. We set

$$
\operatorname{PSH}(X ; \alpha)_{\leq 0}=\{\psi \in \operatorname{PSH}(X ; \alpha) \mid \psi \leq 0\}
$$

By our assumption, we can find a $C^{\infty}$-function $\psi_{0}$ such that $g_{A}+\psi_{0}=h$ (a.e.). Note that $\left[\alpha+d d^{c}\left(\psi_{0}\right)\right]=d d^{c}([h])+\delta_{A}$. Thus $\alpha+d d^{c}\left(\psi_{0}\right)$ is either positive or zero.

First we assume that $\alpha+d d^{c}\left(\psi_{0}\right)$ is positive. Let $g$ be the greatest element of $G_{\mathrm{PSH}}(X ; A)_{\leq g_{A}}$ modulo null functions (cf. Claim 4.6.1). We choose $\phi \in \operatorname{PSH}(X ; \alpha)$ and $\psi_{u} \in \operatorname{PSH}(X ; \alpha)$ for each $u \in G_{\mathrm{PSH}}(X ; A)_{\leq g_{A}}$ such that $g=g_{A}+\phi$ (a.e.) and $u=g_{A}+\psi_{u}$ (a.e.) (cf. Proposition 4.3). Then

$$
\left\{\psi_{u} \mid u \in G_{\mathrm{PSH}}(X ; A)_{\leq g_{A}}\right\}=\operatorname{PSH}(X ; \alpha)_{\leq 0} .
$$

Moreover, by our construction of $g$ (cf. Proposition 4.4 and Claim 4.6.1), $\phi$ is the upper semicontinuous regularization of the function $\phi^{\prime}$ given by

$$
\phi^{\prime}(x)=\sup \left\{\psi_{u}(x) \mid u \in G_{\mathrm{PSH}}(X ; A)_{\leq g_{A}}\right\}\left(=\sup \left\{\psi(x) \mid \psi \in \operatorname{PSH}(X ; \alpha)_{\leq 0}\right\}\right)
$$

for $x \in X$. On the other hand, by [3, Theorem 1.4], $\phi^{\prime}$ is continuous. Thus $\phi=\phi^{\prime}$ and $\phi$ is continuous. Therefore the claim follows by Proposition 4.3.

Next we assume that $\alpha+d d^{c}\left(\psi_{0}\right)=0$, that is, $\alpha=d d^{c}\left(-\psi_{0}\right)$. Then

$$
\operatorname{PSH}(X ; \alpha)=\left\{\psi_{0}+c \mid c \in \mathbb{R} \cup\{-\infty\}\right\}
$$

Let $g$ be the greatest element of $G_{\mathrm{PSH}}(X ; A)_{\leq g_{A}}$ modulo null functions. Then, by Proposition 4.3 , there is $c \in \mathbb{R}$ such that $g=g_{A}+\left(\psi_{0}+c\right)$ (a.e.). Thus the claim follows in this case.

Finally, let us consider the general case. First of all, we may assume $A=B$ as before. We can take a continuous function $f$ on $X$ such that $g_{A}=h+f$ (a.e.). By 
using the Stone-Weierstrass theorem, there is a sequence $\left\{u_{n}\right\}_{n=1}^{\infty}$ of continuous functions on $X$ such that $\lim _{n \rightarrow \infty}\left\|u_{n}\right\|_{\text {sup }}=0$ and $f+u_{n}$ is $C^{\infty}$ for every $n$. Then, as $g_{A}+u_{n}=h+\left(f+u_{n}\right)$ (a.e.), $g_{A}+u_{n}$ is of $C^{\infty}$-type for all $n$. Let $g$ (resp. $\left.g_{n}\right)$ be the greatest element of $G_{\mathrm{PSH}}(X ; A)_{\leq g_{A}}\left(\operatorname{resp} . G_{\mathrm{PSH}}(X ; A)_{\leq g_{A}+u_{n}}\right)$ modulo null functions. Note that the greatest element of $G_{\mathrm{PSH}}(X ; A)_{\leq g_{A} \pm\left\|u_{n}\right\|_{\mathrm{sup}}}$ modulo null functions is given by $g \pm\left\|u_{n}\right\|_{\text {sup }}$. By the previous claim, $g_{n} \in G_{C^{0} \cap \mathrm{PSH}}(X ; A)$. Moreover, since

$$
g_{A}-\left\|u_{n}\right\|_{\sup } \leq g_{A}+u_{n} \leq g_{A}+\left\|u_{n}\right\|_{\text {sup }} \quad \text { (a.e.) }
$$

we have

$$
g-\left\|u_{n}\right\|_{\text {sup }} \leq g_{n} \leq g+\left\|u_{n}\right\|_{\text {sup }} \quad \text { (a.e.) }
$$

for all $n$. Let $g=v+\sum_{i=1}^{l}\left(-a_{i}\right) \log \left|f_{i}\right|^{2}$ (a.e.) and $g_{n}=v_{n}+\sum_{i=1}^{l}\left(-a_{i}\right)\left|f_{i}\right|^{2}$ (a.e.) be local expressions of $g$ and $g_{n}$. Note that $v_{n}$ is continuous for every $n$. By Lemma 2.3.1, $v-\left\|u_{n}\right\|_{\text {sup }} \leq v_{n} \leq v+\left\|u_{n}\right\|_{\text {sup }}$ for all $n$. Thus $v_{n}$ converges to $v$ uniformly, which implies that $v$ is continuous.

(2) Let $\alpha^{\prime}$ be a $C^{\infty}$-form such that $\left[\alpha^{\prime}\right]=d d^{c}([h])+\delta_{A}$. By our assumption, $\alpha^{\prime}$ is either positive or zero. By Proposition 4.3, there is $\psi \in \operatorname{PSH}\left(X ; \alpha^{\prime}\right)$ such that $\psi$ is continuous and $\psi+h=u$ (a.e.). Thus, by Lemma 4.2 , there are sequences $\left\{u_{n}\right\}_{n=1}^{\infty}$ and $\left\{v_{n}\right\}_{n=1}^{\infty}$ of continuous functions on $X$ with the following properties:

(a) $u_{n} \geq 0$ and $v_{n} \geq 0$ for all $n \geq 1$.

(b) $\lim _{n \rightarrow \infty}\left\|u_{n}\right\|_{\text {sup }}=\lim _{n \rightarrow \infty}\left\|v_{n}\right\|_{\text {sup }}=0$.

(c) $\psi-u_{n}, \psi+v_{n} \in \operatorname{PSH}\left(X ; \alpha^{\prime}\right) \cap C^{\infty}(X)$ for every $n \geq 1$.

Note that $u-u_{n}=\left(\psi-u_{n}\right)+h$ (a.e.) and $u+v_{n}=\left(\psi+v_{n}\right)+h$ (a.e.). Therefore, by Proposition 4.3, $u-u_{n}, u+v_{n} \in G_{C^{\infty} \cap \mathrm{PSH}}(X ; A)$.

\section{§5. Arithmetic $\mathbb{R}$-Cartier divisors}

Throughout this section, let $X$ be a $d$-dimensional generically smooth and normal arithmetic variety, that is, $X$ is a flat and quasi-projective integral scheme over $\mathbb{Z}$ such that $X$ is normal, $X$ is smooth over $\mathbb{Q}$ and the Krull dimension of $X$ is $d$.

\section{$\S 5.1$. Definition of arithmetic $\mathbb{R}$-Cartier divisors}

Let $\operatorname{Div}(X)$ be the group of Cartier divisors on $X$. An element of

$$
\operatorname{Div}(X)_{\mathbb{R}}:=\operatorname{Div}(X) \otimes_{\mathbb{Z}} \mathbb{R} \quad\left(\operatorname{resp} \cdot \operatorname{Div}(X)_{\mathbb{Q}}:=\operatorname{Div}(X) \otimes_{\mathbb{Z}} \mathbb{Q}\right)
$$

is called an $\mathbb{R}$-Cartier divisor (resp. $\mathbb{Q}$-Cartier divisor) on $X$. Let $D$ be an $\mathbb{R}$ Cartier divisor on $X$ and let $D=a_{1} D_{1}+\cdots+a_{l} D_{l}$ be the unique decomposition 
of $D$ such that $D_{i}$ 's are prime divisors on $X$ and $a_{1}, \ldots, a_{l} \in \mathbb{R}$. Note that $D_{i}$ 's are not necessarily Cartier divisors on $X$. The $\operatorname{support} \operatorname{Supp}(D)$ of $D$ is defined by $\bigcup_{i \in\left\{i \mid a_{i} \neq 0\right\}} D_{i}$. If $a_{i} \geq 0$ for all $i$, then $D$ is said to be effective and it is denoted by $D \geq 0$. More generally, for $D, E \in \operatorname{Div}(X)_{\mathbb{R}}$, if $D-E \geq 0$, then we write $D \geq E$ or $E \leq D$. We define

$$
H^{0}(X, D)=\left\{\phi \in \operatorname{Rat}(X)^{\times} \mid(\phi)+D \geq 0\right\} \cup\{0\}
$$

where $\operatorname{Rat}(X)$ is the field of rational functions on $X$. Let $F_{\infty}: X(\mathbb{C}) \rightarrow X(\mathbb{C})$ be the complex conjugation map on $X(\mathbb{C})$. Let $g$ be a locally integrable function on $X(\mathbb{C})$. We say $g$ is $F_{\infty}$-invariant if $F_{\infty}^{*}(g)=g$ (a.e.) on $X(\mathbb{C})$. Note that we do not require that $F_{\infty}^{*}(g)$ is identically equal to $g$ on $X(\mathbb{C})$. A pair $\bar{D}=(D, g)$ is called an arithmetic $\mathbb{R}$-Cartier divisor on $X$ if $g$ is $F_{\infty}$-invariant. If $D \in \operatorname{Div}(X)$ (resp. $\left.D \in \operatorname{Div}(X)_{\mathbb{Q}}\right)$, then $\bar{D}$ is called an arithmetic divisor on $X$ (resp. arithmetic $\mathbb{Q}$-Cartier divisor on $X)$. For arithmetic $\mathbb{R}$-Cartier divisors $\bar{D}_{1}=\left(D_{1}, g_{1}\right)$ and $\bar{D}_{2}=\left(D_{2}, g_{2}\right), \bar{D}_{1}=\bar{D}_{2}$ and $\bar{D}_{1} \leq \bar{D}_{2}$ (or $\bar{D}_{2} \geq \bar{D}_{1}$ ) are defined as follows:

$$
\begin{aligned}
& \bar{D}_{1}=\bar{D}_{2} \stackrel{\text { def }}{\Longleftrightarrow} D_{1}=D_{2} \text { and } g_{1}=g_{2} \text { (a.e.), } \\
& \bar{D}_{1} \leq \bar{D}_{2} \stackrel{\text { def }}{\Longleftrightarrow} D_{1} \leq D_{2} \text { and } g_{1} \leq g_{2} \text { (a.e.). }
\end{aligned}
$$

If $\bar{D} \geq(0,0)$, then $\bar{D}$ is said to be arithmetically effective (or effective for simplicity). For arithmetic $\mathbb{R}$-Cartier divisors $\bar{D}$ and $\bar{E}$ on $X$, we define

$$
\begin{aligned}
(-\infty, \bar{D}] & :=\{\bar{M} \mid \bar{M} \text { is an arithmetic } \mathbb{R} \text {-Cartier divisor on } X \text { and } \bar{M} \leq \bar{D}\}, \\
{[\bar{D}, \infty) } & :=\{\bar{M} \mid \bar{M} \text { is an arithmetic } \mathbb{R} \text {-Cartier divisor on } X \text { and } \bar{D} \leq \bar{M}\}, \\
{[\bar{D}, \bar{E}] } & :=\{\bar{M} \mid \bar{M} \text { is an arithmetic } \mathbb{R} \text {-Cartier divisor on } X \text { and } \bar{D} \leq \bar{M} \leq \bar{E}\} .
\end{aligned}
$$

Let $\mathscr{T}$ be a type for Green functions on $X$, that is, $\mathscr{T}$ is a type for Green functions on $X(\mathbb{C})$ together with the following extra $F_{\infty}$-compatibility condition: if $u \in \mathscr{T}(U)$ for an open set $U$ of $X(\mathbb{C})$, then $F_{\infty}^{*}(u) \in \mathscr{T}\left(F_{\infty}^{-1}(U)\right)$. On arithmetic varieties, we always assume the above $F_{\infty}$-compatibility condition for a type for Green functions. We denote the set

$$
\left\{u \in \mathscr{T}(X(\mathbb{C})) \mid u=F_{\infty}^{*}(u)\right\}
$$

by $\mathscr{T}(X)$. Note that $\mathscr{T}(X)$ is different from $\mathscr{T}(X(\mathbb{C}))$. Clearly $C^{0}$ and $C^{\infty}$ have $F_{\infty}$-compatibility. Moreover, by the following lemma, $\mathrm{PSH}$ and $\mathrm{PSH}_{\mathbb{R}}$ also have $F_{\infty}$-compatibility. If two types $\mathscr{T}$ and $\mathscr{T}^{\prime}$ for Green functions have $F_{\infty^{-}}$ compatibility, then so do $\mathscr{T}+\mathscr{T}^{\prime}$ and $\mathscr{T}-\mathscr{T}^{\prime}$.

Lemma 5.1.1. Let $f_{1}, \ldots, f_{r} \in \mathbb{R}\left[X_{1}, \ldots, X_{N}\right]$ and

$$
V=\operatorname{Spec}\left(\mathbb{C}\left[X_{1}, \ldots, X_{N}\right] /\left(f_{1}, \ldots, f_{r}\right)\right) .
$$


Assume that $V$ is e-equidimensional and smooth over $\mathbb{C}$. Let $F_{\infty}: V \rightarrow V$ be the complex conjugation map. If $u$ is a plurisubharmonic function on an open set $U$ of $V$, then $F_{\infty}^{*}(u)$ is a plurisubharmonic function on $F_{\infty}^{-1}(U)$.

Proof. Fix $x \in U$ and choose $i_{1}<\cdots<i_{e}$ such that the projection $p: V \rightarrow \mathbb{C}^{e}$ given by $\left(x_{1}, \ldots, x_{N}\right) \mapsto\left(x_{i_{1}}, \ldots, x_{i_{e}}\right)$ is étale at $x$. Note that the following diagram is commutative:

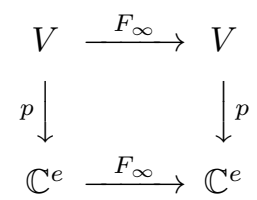

Let $U_{x}$ be an open neighborhood of $x$ such that $\left.p\right|_{U_{x}}: U_{x} \rightarrow W_{x}=p\left(U_{x}\right)$ is an isomorphism of complex manifolds. Then $\left.p\right|_{F_{\infty}^{-1}\left(U_{x}\right)}: F_{\infty}^{-1}\left(U_{x}\right) \rightarrow F_{\infty}^{-1}\left(W_{x}\right)$ is also an isomorphism as complex manifolds. This observation indicates that we may assume $V=\mathbb{C}^{e}$.

Let $y \in F_{\infty}^{-1}(U) \subseteq \mathbb{C}^{e}$ and $\xi \in \mathbb{C}^{e}$ be such that $y+\xi \exp (\sqrt{-1} \theta) \in F_{\infty}^{-1}(U)$ for all $0 \leq \theta \leq 2 \pi$. Then

$$
\begin{aligned}
F_{\infty}^{*}(u)(y) & =u(\bar{y}) \leq \frac{1}{2 \pi} \int_{0}^{2 \pi} u(\bar{y}+\bar{\xi} \exp (\sqrt{-1} \theta)) d \theta \\
& =\frac{1}{2 \pi} \int_{0}^{2 \pi} u(\bar{y}+\bar{\xi} \exp (-\sqrt{-1} \theta)) d \theta=\frac{1}{2 \pi} \int_{0}^{2 \pi} u(\overline{y+\xi \exp (\sqrt{-1} \theta)}) d \theta \\
& =\frac{1}{2 \pi} \int_{0}^{2 \pi} F_{\infty}^{*}(u)(y+\xi \exp (\sqrt{-1} \theta)) d \theta
\end{aligned}
$$

which shows that $F_{\infty}^{*}(u)$ is plurisubharmonic on $F_{\infty}^{-1}(U)$.

Let $D$ be an $\mathbb{R}$-Cartier divisor on $X$ and let $g$ be a $D$-Green function on $X(\mathbb{C})$. By the following lemma, $\frac{1}{2}\left(g+F_{\infty}^{*}(g)\right)$ is an $F_{\infty}$-invariant $D$-Green function of $\mathscr{T}$ type on $X(\mathbb{C})$.

Lemma 5.1.2. If $g$ is a D-Green function of $\mathscr{T}$-type, then so is $F_{\infty}^{*}(g)$.

Proof. Let $D=a_{1} D_{1}+\cdots+a_{l} D_{l}$ be a decomposition of $D$ such that $a_{1}, \ldots, a_{l} \in \mathbb{R}$ and $D_{i}$ 's are Cartier divisors on $X$. Let $U$ be a Zariski open set of $X$ over which $D_{i}$ can be written by a local equation $\phi_{i}$ for each $i$. Let $g=u+\sum_{i=1}^{l}\left(-a_{i}\right) \log \left|\phi_{i}\right|^{2}$ (a.e.) be the local expression of $g$ with respect to $\phi_{1}, \ldots, \phi_{l}$ over $U(\mathbb{C})$. Note that $F_{\infty}^{*}\left(\phi_{i}\right)=\bar{\phi}_{i}$ as a function over $U(\mathbb{C})$. Thus $F_{\infty}^{*}(g)=F_{\infty}^{*}(u)+\sum_{i=1}^{l}\left(-a_{i}\right) \log \left|\phi_{i}\right|^{2}$ (a.e.) is a local expression of $F_{\infty}^{*}(g)$, as required. 
We define

$$
\begin{gathered}
\widehat{\operatorname{Div}}_{\mathscr{T}}(X):=\left\{(D, g) \mid \begin{array}{l}
D \in \operatorname{Div}(X) \text { and } g \text { is an } F_{\infty} \text {-invariant } \\
D \text {-Green function of } \mathscr{T} \text {-type on } X(\mathbb{C})
\end{array}\right\}, \\
\widehat{\operatorname{Div}} \mathscr{T}(X)_{\mathbb{Q}}:=\left\{(D, g) \mid \begin{array}{l}
D \in \operatorname{Div}(X)_{\mathbb{Q}} \text { and } g \text { is an } F_{\infty} \text {-invariant } \\
D \text {-Green function of } \mathscr{T} \text {-type on } X(\mathbb{C})
\end{array}\right\}, \\
\widehat{\operatorname{Div}} \mathscr{T}(X)_{\mathbb{R}}:=\left\{(D, g) \mid \begin{array}{l}
D \in \operatorname{Div}(X)_{\mathbb{R}} \text { and } g \text { is an } F_{\infty} \text {-invariant } \\
D \text {-Green function of } \mathscr{T} \text {-type on } X(\mathbb{C})
\end{array}\right\} .
\end{gathered}
$$

An element of $\widehat{\operatorname{Div}}_{\mathscr{T}}(X)_{\mathbb{R}}$ (resp. $\left.\widehat{\operatorname{Div}}_{\mathscr{T}}(X)_{\mathbb{Q}}, \widehat{\operatorname{Div}}_{\mathscr{T}}(X)\right)$ is called an arithmetic $\mathbb{R}$-Cartier divisor of $\mathscr{T}$-type on $X$ (resp. arithmetic $\mathbb{Q}$-Cartier divisor of $\mathscr{T}$-type on $X$, arithmetic Cartier divisor of $\mathscr{T}$-type on $X)$. Let $\bar{D}=(D, g)$ be an arithmetic $\mathbb{R}$-Cartier divisor of $\mathscr{T}$-type. Then, as $F_{\infty}^{*}(g)=g$ (a.e.), we can see that $F_{\infty}^{*}\left(g_{\text {can }}\right)=g_{\text {can }}$ on $X(\mathbb{C}) \backslash \operatorname{Supp}(D)(\mathbb{C})$.

Now we recall $\widehat{\mathrm{Pic}}_{C^{0}}(X), \widehat{\mathrm{Pic}}_{C^{0}}(X)_{\mathbb{Q}}$ and $\widehat{\mathrm{Pic}}_{C^{0}}(X)_{\mathbb{R}}$ (for details, see [15]). First of all, let $\widehat{\mathrm{Pic}}_{C^{0}}(X)$ be the group of isomorphism classes of $F_{\infty}$-invariant continuous hermitian invertible sheaves on $X$ and let $\widehat{\operatorname{Pic}}_{C^{0}}(X)_{\mathbb{Q}}:=\widehat{\operatorname{Pic}}_{C^{0}}(X) \otimes_{\mathbb{Z}} \mathbb{Q}$. For an $F_{\infty}$-invariant continuous function $f$ on $X(\mathbb{C}), \overline{\mathscr{O}}(f)$ is $\left(\mathscr{O}_{X}, \exp (-f)|\cdot|_{\text {can }}\right)$. Then we define

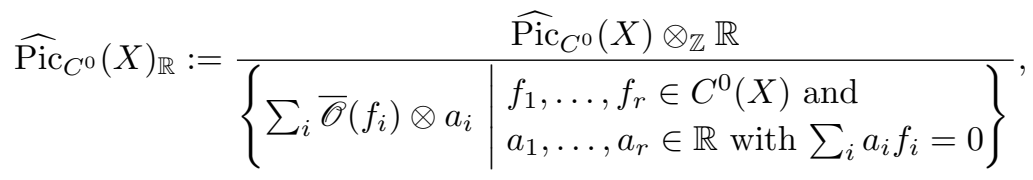

where $C^{0}(X)=\left\{f \in C^{0}(X(\mathbb{C})) \mid F_{\infty}^{*}(f)=f\right\}$ as before. Note that there is a natural surjective homomorphism $\overline{\mathscr{O}}: \widehat{\operatorname{Div}}_{C^{0}}(X) \rightarrow \widehat{\operatorname{Pic}}_{C^{0}}(X)$ given by

$$
\overline{\mathscr{O}}(D, g)=\left(\mathscr{O}_{X}(D),|\cdot|_{g}\right)
$$

where $|1|_{g}=\exp (-g / 2)$.

\section{$\S 5.2$. Volume function for arithmetic $\mathbb{R}$-Cartier divisors}

We assume that $X$ is projective. Let $\bar{D}=(D, g)$ be an arithmetic $\mathbb{R}$-Cartier divisor on $X$. We set

$$
\hat{H}^{0}(X, \bar{D})=\left\{\phi \in H^{0}(X, D) \mid\|\phi\|_{g} \leq 1\right\}
$$

and

$$
\hat{h}^{0}(X, \bar{D})= \begin{cases}\log \# \hat{H}^{0}(X, \bar{D}) & \text { if } \hat{H}^{0}(X, \bar{D}) \text { is finite } \\ \infty & \text { otherwise }\end{cases}
$$


where $\|\phi\|_{g}$ is the essential supremum of $|\phi|_{g}=|\phi| \exp (-g / 2)$. Note that

$$
\hat{H}^{0}(X, \bar{D})=\left\{\phi \in \operatorname{Rat}(X)^{\times} \mid \widehat{(\phi)}+\bar{D} \geq 0\right\} \cup\{0\} .
$$

The volume $\widehat{\operatorname{vol}}(\bar{D})$ of $\bar{D}$ is defined to be

$$
\widehat{\operatorname{vol}}(\bar{D})=\limsup _{n \rightarrow \infty} \frac{\hat{h}^{0}(X, n \bar{D})}{n^{d} / d !} .
$$

For arithmetic $\mathbb{R}$-Cartier divisors $\bar{D}$ and $\bar{D}^{\prime}$ on $X$, if $\bar{D} \leq \bar{D}^{\prime}$, then $\hat{H}^{0}(X, \bar{D}) \subseteq$ $\hat{H}^{0}\left(X, \bar{D}^{\prime}\right)$ and $\widehat{\operatorname{vol}}(\bar{D}) \leq \widehat{\operatorname{vol}}\left(\bar{D}^{\prime}\right)$.

Proposition 5.2.1. Let $\mathscr{T}$ be a type for Green functions on $X$ and let $\bar{D}=(D, g)$ be an arithmetic $\mathbb{R}$-Cartier divisor of $\mathscr{T}$-type on $X$. If $g$ is either of upper bounded type or of lower bounded type, then $\hat{H}^{0}(X, \bar{D})$ is finite. Moreover, if $g$ is of upper bounded type, then $\widehat{\operatorname{vol}}(\bar{D})<\infty$.

Proof. First we assume that $g$ is of lower bounded type. Then, by Lemma 2.5.1, $\|\cdot\|_{g}$ yields a norm on $H^{0}(X, D)$, and hence the assertion follows.

Next we assume that $g$ is of upper bounded type. Then, by Proposition 2.3.6, there is an $F_{\infty}$-invariant $D$-Green function $g^{\prime}$ of $C^{\infty}$-type such that $g \leq g^{\prime}$ (a.e.). By Proposition 2.4.2, we can choose $a_{1}, \ldots, a_{l} \in \mathbb{R}$ and $\bar{D}_{1}, \ldots, \bar{D}_{l} \in \widehat{\operatorname{Div}}_{C^{\infty}}(X)$ such that $\left(D, g^{\prime}\right)=a_{1} \bar{D}_{1}+\cdots+a_{l} \bar{D}_{l}$. For each $i$, by using Lemmas 5.2.3 and 5.2.4 below, we can find effective arithmetic Cartier divisors $\bar{A}_{i}$ and $\bar{B}_{i}$ of $C^{\infty}$-type such that $\bar{D}_{i}=\bar{A}_{i}-\bar{B}_{i}$. As

$$
\left(D, g^{\prime}\right)=a_{1} \bar{A}_{1}+\cdots+a_{l} \bar{A}_{l}+\left(-a_{1}\right) \bar{B}_{1}+\cdots+\left(-a_{l}\right) \bar{B}_{l},
$$

if we set $\bar{D}^{\prime \prime}=\left\lceil a_{1}\right\rceil \bar{A}_{1}+\cdots+\left\lceil a_{l}\right\rceil \bar{A}_{l}+\left\lceil\left(-a_{1}\right)\right\rceil \bar{B}_{1}+\cdots+\left\lceil\left(-a_{l}\right)\right\rceil \bar{B}_{l}$, then $\left(D, g^{\prime}\right) \leq$ $\bar{D}^{\prime \prime}$ and $\bar{D}^{\prime \prime} \in \widehat{\operatorname{Div}}_{C^{\infty}}(X)$. Note that

$$
\hat{H}^{0}(X, n \bar{D}) \subseteq \hat{H}^{0}\left(X, n\left(D, g^{\prime}\right)\right) \subseteq \hat{H}^{0}\left(X, n \bar{D}^{\prime \prime}\right)=\hat{H}^{0}\left(X, \overline{\mathscr{O}}\left(\bar{D}^{\prime \prime}\right)^{\otimes n}\right)
$$

for all $n \geq 1$. Thus our assertion follows from [14, Lemma 3.3].

Now we consider the fundamental properties of $\widehat{\operatorname{vol}}$ on $\widehat{\operatorname{Div}}_{C^{0}}(X)_{\mathbb{R}}$.

Theorem 5.2.2. There is a natural surjective homomorphism

$$
\overline{\mathscr{O}}_{\mathbb{R}}: \widehat{\operatorname{Div}}_{C^{0}}(X)_{\mathbb{R}} \rightarrow \widehat{\operatorname{Pic}}_{C^{0}}(X)_{\mathbb{R}}
$$

such that the following diagram is commutative:

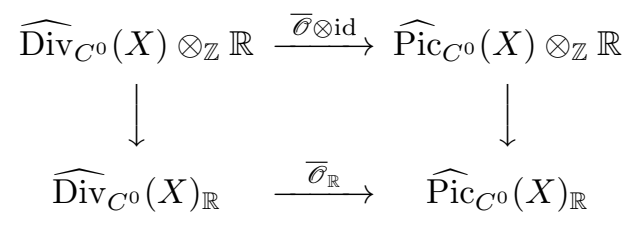


Moreover, we have the following:

(1) For all $\bar{D} \in \widehat{\operatorname{Div}}_{C^{0}}(X)_{\mathbb{R}}$,

$$
\widehat{\operatorname{vol}}(\bar{D})=\lim _{t \rightarrow \infty} \frac{\hat{h}^{0}(t \bar{D})}{t^{d} / d !}=\widehat{\operatorname{vol}}\left(\overline{\mathscr{O}}_{\mathbb{R}}(\bar{D})\right),
$$

where $t \in \mathbb{R}_{>0}$ and $\widehat{\operatorname{vol}}\left(\overline{\mathscr{O}}_{\mathbb{R}}(\bar{D})\right)$ is the volume defined in $[15$, Section 4$]$.

(2) $\widehat{\operatorname{vol}}(a \bar{D})=a^{d} \widehat{\operatorname{vol}}(\bar{D})$ for all $a \in \mathbb{R}_{\geq 0}$ and $\bar{D} \in \widehat{\operatorname{Div}}_{C^{0}}(X)_{\mathbb{R}}$.

(3) (Continuity of $\widehat{\mathrm{vol}}$ ) Let $\bar{D}_{1}, \ldots, \bar{D}_{r}, \bar{A}_{1}, \ldots, \bar{A}_{r^{\prime}} \in \widehat{\operatorname{Div}}_{C^{0}}(X)_{\mathbb{R}}$. For a compact set $B$ in $\mathbb{R}^{r}$ and a positive number $\epsilon$, there are positive numbers $\delta$ and $\delta^{\prime}$ such that, for all $a_{1}, \ldots, a_{r}, \delta_{1}, \ldots, \delta_{r^{\prime}} \in \mathbb{R}$ and $\phi \in C^{0}(X)$ with $\left(a_{1}, \ldots, a_{r}\right) \in B$, $\sum_{j=1}^{r^{\prime}}\left|\delta_{j}\right| \leq \delta$ and $\|\phi\|_{\text {sup }} \leq \delta^{\prime}$, we have

$$
\left|\widehat{\operatorname{vol}}\left(\sum_{i=1}^{r} a_{i} \bar{D}_{i}+\sum_{j=1}^{r^{\prime}} \delta_{j} \bar{A}_{j}+(0, \phi)\right)-\widehat{\operatorname{vol}}\left(\sum_{i=1}^{r} a_{i} \bar{D}_{i}\right)\right| \leq \epsilon .
$$

Moreover, if $\bar{D}_{1}, \ldots, \bar{D}_{r}, \bar{A}_{1}, \ldots, \bar{A}_{r^{\prime}}$ are $C^{\infty}$, then there is a positive constant $C$ depending only on $X$ and $\bar{D}_{1}, \ldots, \bar{D}_{r}, \bar{A}_{1}, \ldots, \bar{A}_{r^{\prime}}$ such that

$$
\begin{array}{r}
\left|\widehat{\operatorname{vol}}\left(\sum_{i=1}^{r} a_{i} \bar{D}_{i}+\sum_{j=1}^{r^{\prime}} \delta_{j} \bar{A}_{j}+(0, \phi)\right)-\widehat{\operatorname{vol}}\left(\sum_{i=1}^{r} a_{i} \bar{D}_{i}\right)\right| \\
\leq C\left(\sum_{i=1}^{r}\left|a_{i}\right|+\sum_{j=1}^{r^{\prime}}\left|\delta_{j}\right|\right)^{d-1}\left(\|\phi\|_{\text {sup }}+\sum_{j=1}^{r^{\prime}}\left|\delta_{j}\right|\right)
\end{array}
$$

for all $a_{1}, \ldots, a_{r}, \delta_{1}, \ldots, \delta_{r^{\prime}} \in \mathbb{R}$ and $\phi \in C^{0}(X)$.

(4) Let $\bar{D}_{1}$ and $\bar{D}_{2}$ be arithmetic $\mathbb{R}$-Cartier divisors of $C^{0}$-type. If $\bar{D}_{1}$ and $\bar{D}_{2}$ are pseudo-effective (for the definition, see Subsection 6.1), then

$$
\widehat{\operatorname{vol}}\left(\bar{D}_{1}+\bar{D}_{2}\right)^{1 / d} \geq \widehat{\operatorname{vol}}\left(\bar{D}_{1}\right)^{1 / d}+\widehat{\operatorname{vol}}\left(\bar{D}_{2}\right)^{1 / d} .
$$

(5) (Fujita's approximation theorem for arithmetic $\mathbb{R}$-Cartier divisors) If $\bar{D}$ is an arithmetic $\mathbb{R}$-Cartier divisor of $C^{0}$-type and $\widehat{\operatorname{vol}}(\bar{D})>0$, then, for any positive number $\epsilon$, there are a birational morphism $\mu: Y \rightarrow X$ of generically smooth and normal projective arithmetic varieties and an ample arithmetic $\mathbb{Q}$-Cartier divisor $\bar{A}$ of $C^{\infty}$-type on $Y$ (cf. Section 6) such that $\bar{A} \leq \mu^{*}(\bar{D})$ and $\widehat{\operatorname{vol}}(\bar{A}) \geq \widehat{\operatorname{vol}}(\bar{D})-\epsilon$.

Let us begin with the following lemmas.

Lemma 5.2.3. Let $Y$ be a normal projective arithmetic variety. Then we have the following: 
(1) Let $Z$ be a Weil divisor on $Y$. Then there is an effective Cartier divisor $A$ on $Y$ such that $Z \leq A$.

(2) Let $D$ be a Cartier divisor on $Y$. Then there are effective Cartier divisors $A$ and $B$ on $Y$ such that $D=A-B$.

(3) Let $x_{1}, \ldots, x_{l}$ be points of $Y$ and let $D$ be a Cartier divisor on $Y$. Then there are effective Cartier divisors $A$ and $B$, and a non-zero rational function $\phi$ on $Y$ such that $D+(\phi)=A-B$ and $x_{1}, \ldots, x_{l} \notin \operatorname{Supp}(A) \cup \operatorname{Supp}(B)$.

Proof. (1) Let $Z=a_{1} \Gamma_{1}+\cdots+a_{l} \Gamma_{l}$ be the decomposition such that $\Gamma_{i}$ 's are prime divisors on $Y$ and $a_{1}, \ldots, a_{l} \in \mathbb{Z}$. Let $L$ be an ample invertible sheaf on $Y$. Then we can choose a positive integer $n$ and a non-zero section $s \in H^{0}\left(Y, L^{\otimes n}\right)$ such that $\operatorname{mult}_{\Gamma_{i}}(s) \geq a_{i}$ for all $i$. Thus, if we set $A=\operatorname{div}(s)$, then $A$ is a Cartier divisor and $Z \leq A$.

(2) First of all, we can find effective Weil divisors $A^{\prime}$ and $B^{\prime}$ on $Y$ such that $D=A^{\prime}-B^{\prime}$. By (1), there is an effective Cartier divisor $A$ such that $A^{\prime} \leq A$. We set $B=B^{\prime}+\left(A-A^{\prime}\right)$. Then $B$ is effective and $D=A-B$. Moreover, since $B=A-D, B$ is a Cartier divisor.

(3) Let $L$ be an ample invertible sheaf on $Y$ as before. Then there are a positive integer $n_{1}$ and a non-zero $s_{1} \in H^{0}\left(Y, L^{\otimes n_{1}}\right)$ such that $s_{1}\left(x_{i}\right) \neq 0$ for all $i$. We set $A^{\prime}=\operatorname{div}\left(s_{1}\right)$. Similarly we can find a positive integer $n_{2}$ and a non-zero $s_{2} \in H^{0}\left(Y, \mathscr{O}_{Y}\left(n_{2} A^{\prime}-D\right)\right)$ such that $s_{2}\left(x_{i}\right) \neq 0$ for all $i$. Therefore, if we set $A=n_{2} A^{\prime}$ and $B=\operatorname{div}\left(s_{2}\right)$, then there is a non-zero rational function $\phi$ on $Y$ such that $A-D=B+(\phi)$, as required.

Lemma 5.2.4. Let $\mathscr{T}$ be either $C^{0}$ or $C^{\infty}$. Let $A^{\prime}$ and $A^{\prime \prime}$ be effective $\mathbb{R}$-Cartier divisors on $X$ and $A=A^{\prime}-A^{\prime \prime}$. Let $g_{A}$ be an $F_{\infty}$-invariant $A$-Green function of $\mathscr{T}$-type on $X(\mathbb{C})$. Then there are effective arithmetic $\mathbb{R}$-Cartier divisors $\left(A^{\prime}, g_{A^{\prime}}\right)$ and $\left(A^{\prime \prime}, g_{A^{\prime \prime}}\right)$ of $\mathscr{T}$-type such that $\left(A, g_{A}\right)=\left(A^{\prime}, g_{A^{\prime}}\right)-\left(A^{\prime \prime}, g_{A^{\prime \prime}}\right)$.

Proof. Let $g_{A^{\prime \prime}}$ be an $F_{\infty}$-invariant $A^{\prime \prime}$-Green function of $\mathscr{T}$-type such that $g_{A^{\prime \prime}}$ $\geq 0$ (a.e.). We put $g_{A^{\prime}}=g_{A}+g_{A^{\prime \prime}}$. Then $g_{A^{\prime}}$ is an $F_{\infty}$-invariant $A^{\prime}$-Green function of $\mathscr{T}$-type. Replacing $g_{A^{\prime \prime}}$ with $g_{A^{\prime \prime}}+$ (positive constant) if necessary, we have $g_{A^{\prime}} \geq 0$ (a.e.).

Lemma 5.2.5. Let $\mathscr{T}$ be a type for Green functions such that $-\mathscr{T} \subseteq \mathscr{T}$ and $C^{\infty} \subseteq \mathscr{T}$. Then the kernel of the natural homomorphism $\widehat{\operatorname{Div}}_{\mathscr{T}}(X) \otimes_{\mathbb{Z}} \mathbb{R} \rightarrow$ $\widehat{\operatorname{Div}} \mathscr{T}(X)_{\mathbb{R}}$ coincides with

$$
\left\{\begin{array}{l|l}
\sum_{i=1}^{l}\left(0, \phi_{i}\right) \otimes a_{i} & \begin{array}{l}
a_{1}, \ldots, a_{l} \in \mathbb{R}, \phi_{1}, \ldots, \phi_{l} \in \mathscr{T}(X) \\
\text { and } a_{1} \phi_{1}+\cdots+a_{l} \phi_{l}=0
\end{array}
\end{array}\right\} .
$$


Proof. It is sufficient to show that, for $\sum_{i=1}^{l}\left(D_{i}, g_{i}\right) \otimes a_{i} \in \widehat{\operatorname{Div}} \mathscr{T}(X) \otimes_{\mathbb{Z}} \mathbb{R}$, if

$$
\sum_{i=1}^{l} a_{i} D_{i}=0 \text { and } \sum_{i=1}^{l} a_{i} g_{i}=0 \text { (a.e.) }
$$

then there are $\phi_{1}, \ldots, \phi_{l} \in \mathscr{T}(X)$ such that $\sum_{i=1}^{l}\left(D_{i}, g_{i}\right) \otimes a_{i}=\sum_{i=1}^{l}\left(0, \phi_{i}\right) \otimes a_{i}$ and $a_{1} \phi_{1}+\cdots+a_{l} \phi_{l}=0$. Let $E_{1}, \ldots, E_{r}$ be a free basis of the $\mathbb{Z}$-submodule of $\operatorname{Div}(X)$ generated by $D_{1}, \ldots, D_{l}$. We set $D_{i}=\sum_{j=1}^{r} b_{i j} E_{j}$. Since

$$
0=\sum_{i=1}^{l} a_{i} D_{i}=\sum_{j=1}^{r}\left(\sum_{i=1}^{l} a_{i} b_{i j}\right) E_{j}
$$

we have $\sum_{i=1}^{l} a_{i} b_{i j}=0$ for all $j=1, \ldots, r$. Let $h_{j}$ be an $F_{\infty}$-invariant $E_{j}$-Green function of $C^{\infty}$-type. Note that $\sum_{j=1}^{r} b_{i j} h_{j}$ is an $F_{\infty}$-invariant $D_{i}$-Green function of $\mathscr{T}$-type. Thus we can find $\phi_{1}, \ldots, \phi_{l} \in \mathscr{T}(X)$ such that

$$
\left.g_{i}=\sum_{j=1}^{r} b_{i j} h_{j}+\phi_{i} \quad \text { (a.e. }\right)
$$

for each $i$. Then

$$
0 \stackrel{\text { (a.e.) }}{=} \sum_{i=1}^{l} a_{i} g_{i} \stackrel{\text { (a.e.) }}{=} \sum_{j=1}^{r}\left(\sum_{i=1}^{l} a_{i} b_{i j}\right) h_{j}+\sum_{i=1}^{l} a_{i} \phi_{i}=\sum_{i=1}^{l} a_{i} \phi_{i} \text {. }
$$

Note that $\sum_{i} a_{i} \phi_{i} \in \mathscr{T}(X)$, so that $\sum_{i} a_{i} \phi_{i}=0$ over $X(\mathbb{C})$. On the other hand,

$$
\begin{aligned}
\sum_{i=1}^{l}\left(D_{i}, g_{i}\right) \otimes a_{i} & =\sum_{i=1}^{l} \sum_{j=1}^{r}\left(E_{j}, h_{j}\right) \otimes a_{i} b_{i j}+\sum_{i=1}^{l}\left(0, \phi_{i}\right) \otimes a_{i} \\
& =\sum_{j=1}^{r}\left(E_{j}, h_{j}\right) \otimes\left(\sum_{i=1}^{l} a_{i} b_{i j}\right)+\sum_{i=1}^{l}\left(0, \phi_{i}\right) \otimes a_{i}=\sum_{i=1}^{l}\left(0, \phi_{i}\right) \otimes a_{i}
\end{aligned}
$$

as required.

Proof of Theorem 5.2.2. By Proposition 2.4.2, the natural homomorphism

$$
\widehat{\operatorname{Div}}_{C^{0}}(X) \otimes_{\mathbb{Z}} \mathbb{R} \rightarrow \widehat{\operatorname{Div}}_{C^{0}}(X)_{\mathbb{R}}
$$

is surjective. Thus the first assertion follows from Lemma 5.2.5.

(1) We set $\bar{D}=a_{1} \bar{D}_{1}+\cdots+a_{l} \bar{D}_{l}$, where $a_{1}, \ldots, a_{l} \in \mathbb{R}$ and $\bar{D}_{1}, \ldots, \bar{D}_{l} \in$ $\widehat{\operatorname{Div}}_{C^{0}}(X)$. For each $\bar{D}_{i}$, by using Lemmas 5.2 .3 and 5.2 .4 , we can find effective arithmetic Cartier divisors $\bar{D}_{i}^{\prime}$ and $\bar{D}_{i}^{\prime \prime}$ of $C^{0}$-type such that $\bar{D}_{i}=\bar{D}_{i}^{\prime}-\bar{D}_{i}^{\prime \prime}$. Then

$$
\bar{D}=a_{1} \bar{D}_{1}^{\prime}+\cdots+a_{l} \bar{D}_{l}^{\prime}+\left(-a_{1}\right) \bar{D}_{1}^{\prime \prime}+\cdots+\left(-a_{l}\right) \bar{D}_{l}^{\prime \prime} .
$$


Thus we may assume that $\bar{D}_{i}$ is effective for every $i$. We set $I=\left\{i \mid a_{i} \geq 0\right\}$ and $J=\left\{i \mid a_{i}<0\right\}$. Moreover, we set

$$
\begin{aligned}
& \bar{A}_{n}=\sum_{i \in I}\left\lfloor n a_{i}\right\rfloor \bar{D}_{i}+\sum_{j \in J}\left\lfloor(n+1) a_{j}\right\rfloor \bar{D}_{j}, \\
& \bar{B}_{n}=\sum_{i \in I}\left\lceil n a_{i}\right\rceil \bar{D}_{i}+\sum_{j \in J}\left\lceil(n-1) a_{j}\right\rceil \bar{D}_{j}
\end{aligned}
$$

for $n \in \mathbb{Z}_{\geq 1}$. Then, as $\lim _{n \rightarrow \infty} \bar{A}_{n} / n=\lim _{n \rightarrow \infty} \bar{B}_{n} / n=\bar{D}$, by [15, Theorem 5.1] we have

$$
\lim _{n \rightarrow \infty} \frac{\hat{h}^{0}\left(X, \bar{A}_{n}\right)}{n^{d} / d !}=\lim _{n \rightarrow \infty} \frac{\hat{h}^{0}\left(X, \bar{B}_{n}\right)}{n^{d} / d !}=\widehat{\operatorname{vol}}\left(\overline{\mathscr{O}}_{\mathbb{R}}(\bar{D})\right)
$$

Note that

$$
\begin{cases}\lfloor\lfloor t\rfloor a\rfloor \leq t a \leq\lceil\lceil t\rceil a\rceil & \text { if } a \geq 0 \\ \lfloor(\lfloor t\rfloor+1) a\rfloor \leq t a \leq\lceil(\lceil t\rceil-1) a\rceil & \text { if } a<0\end{cases}
$$

for $a \in \mathbb{R}$ and $t \in \mathbb{R}_{\geq 1}$, which yields $\bar{A}_{\lfloor t\rfloor} \leq t \bar{D} \leq \bar{B}_{\lceil t\rceil}$ for $t \in \mathbb{R}_{\geq 1}$. Therefore,

$$
\frac{(\lfloor t\rfloor)^{d}}{t^{d}} \cdot \frac{h^{0}\left(X, \bar{A}_{\lfloor t\rfloor}\right)}{(\lfloor t\rfloor)^{d} / d !} \leq \frac{h^{0}(X, t \bar{D})}{t^{d} / d !} \leq \frac{(\lceil t\rceil)^{d}}{t^{d}} \cdot \frac{h^{0}\left(X, \bar{B}_{\lceil t\rceil}\right)}{(\lceil t\rceil)^{d} / d !},
$$

and hence (1) follows.

(2) follows from (1).

(3) The first assertion follows from [15, (4) in Proposition 4.6]. Let us prove the second assertion. We choose $\bar{E}_{1}, \ldots, \bar{E}_{m}, \bar{B}_{1}, \ldots, \bar{B}_{m^{\prime}} \in \widehat{\operatorname{Div}}_{C^{\infty}}(X)$ such that $\bar{D}_{i}=\sum_{k=1}^{m} \alpha_{i k} \bar{E}_{k}$ and $\bar{A}_{j}=\sum_{l=1}^{m^{\prime}} \beta_{j l} \bar{B}_{l}$ for some $\alpha_{i k}, \beta_{j l} \in \mathbb{R}$. Then

$$
\sum_{i=1}^{r} a_{i} \bar{D}_{i}=\sum_{k=1}^{m}\left(\sum_{i=1}^{r} a_{i} \alpha_{i k}\right) \bar{E}_{k} \quad \text { and } \quad \sum_{j=1}^{r^{\prime}} \delta_{j} \bar{A}_{j}=\sum_{l=1}^{m^{\prime}}\left(\sum_{j=1}^{r^{\prime}} \delta_{j} \beta_{j l}\right) \bar{B}_{l} .
$$

Moreover, if we set $C^{\prime}=\max \left(\left\{\alpha_{i k}\right\} \cup\left\{\beta_{j l}\right\}\right)$, then

$$
\left|\sum_{i=1}^{r} a_{i} \alpha_{i k}\right| \leq C^{\prime} \sum_{i=1}^{r}\left|a_{i}\right| \text { and }\left|\sum_{j=1}^{r^{\prime}} \delta_{j} \beta_{j l}\right| \leq C^{\prime} \sum_{j=1}^{r^{\prime}}\left|\delta_{j}\right| .
$$

Thus we may assume that $\bar{D}_{1}, \ldots, \bar{D}_{r}, \bar{A}_{1}, \ldots, \bar{A}_{r^{\prime}} \in \widehat{\operatorname{Div}}_{C^{\infty}}(X)$. Therefore, the second assertion of (3) follows from [15, Lemma 3.1, Theorem 4.4 and Proposition 4.6].

(4) If $\widehat{\operatorname{vol}}\left(\bar{D}_{1}\right)>0$ and $\widehat{\operatorname{vol}}\left(\bar{D}_{2}\right)>0$, then (4) follows from (3) and [23, Theorem B] (or [16, Theorem 6.2]). Let us fix an ample arithmetic Cartier divisor $\bar{A}$ (for the definition of ampleness, see Subsection 6.1). Then $\widehat{\operatorname{vol}}\left(\bar{D}_{1}+\epsilon \bar{A}\right)>0$ and 
$\widehat{\operatorname{vol}}\left(\bar{D}_{2}+\epsilon \bar{A}\right)>0$ for all $\epsilon>0$ by Proposition 6.3.2. Thus, by using (3) and the previous observation, we obtain (4).

(5) By using the continuity of vol and the Stone-Weierstrass theorem, we can find an arithmetic $\mathbb{Q}$-Cartier divisor $\bar{D}^{\prime}$ of $C^{\infty}$-type such that $\bar{D}^{\prime} \leq \bar{D}$ and

$$
\widehat{\operatorname{vol}}\left(\bar{D}^{\prime}\right)>\max \{\widehat{\operatorname{vol}}(\bar{D})-\epsilon / 2,0\} .
$$

Then, by virtue of [6], [23] or [16], there are a birational morphism $\mu: Y \rightarrow X$ of generically smooth and normal projective arithmetic varieties and an ample arithmetic $\mathbb{Q}$-Cartier divisor $\bar{A}$ of $C^{\infty}$-type on $Y$ such that $\bar{A} \leq \mu^{*}\left(\bar{D}^{\prime}\right)$ and $\widehat{\operatorname{vol}}(\bar{A}) \geq \widehat{\operatorname{vol}}\left(\bar{D}^{\prime}\right)-\epsilon / 2$. Thus (5) follows.

\section{§5.3. Intersection number of arithmetic $\mathbb{R}$-Cartier divisors with a 1-dimensional subscheme}

We assume that $X$ is projective. Let $C$ be a 1-dimensional closed integral subscheme of $X$. Let $\overline{\mathcal{L}}=(\mathcal{L}, h)$ be an $F_{\infty}$-invariant continuous hermitian invertible sheaf on $X$. Then it is well-known that $\widehat{\operatorname{deg}}\left(\left.\overline{\mathcal{L}}\right|_{C}\right)$ is defined and it has the following property: if $s$ is a non-zero element of $H^{0}(X, \mathcal{L})$ with $\left.s\right|_{C} \neq 0$, then

$$
\widehat{\operatorname{deg}}\left(\left.\overline{\mathcal{L}}\right|_{C}\right)=\log \#\left(\frac{\left.\mathcal{L}\right|_{C}}{\mathscr{O}_{C} \cdot s}\right)-\frac{1}{2} \sum_{x \in C(\mathbb{C})} \log (h(s, s)(x)) .
$$

In addition, the map

$$
\widehat{\operatorname{Pic}}_{C^{0}}(X) \rightarrow \mathbb{R} \quad\left(\overline{\mathcal{L}} \mapsto \widehat{\operatorname{deg}}\left(\left.\overline{\mathcal{L}}\right|_{C}\right)\right)
$$

is a homomorphism of abelian groups, so that it extends to a homomorphism

$$
\widehat{\operatorname{deg}}\left(-\left.\right|_{C}\right): \widehat{\operatorname{Pic}}_{C^{0}}(X) \otimes_{\mathbb{Z}} \mathbb{R} \rightarrow \mathbb{R}
$$

given by

$$
\widehat{\operatorname{deg}}\left(\left.\left(\overline{\mathcal{L}}_{1} \otimes a_{1}+\cdots+\overline{\mathcal{L}}_{r} \otimes a_{r}\right)\right|_{C}\right)=a_{1} \widehat{\operatorname{deg}}\left(\left.\overline{\mathcal{L}}_{1}\right|_{C}\right)+\cdots+a_{r} \widehat{\operatorname{deg}}\left(\left.\overline{\mathcal{L}}_{r}\right|_{C}\right) .
$$

If $f_{1}, \ldots, f_{r} \in C^{0}(X), a_{1}, \ldots, a_{r} \in \mathbb{R}$ and $a_{1} f_{1}+\cdots+a_{r} f_{r}=0$, then

$$
\begin{aligned}
\widehat{\operatorname{deg}}\left(\left.\left(\overline{\mathscr{O}}\left(f_{1}\right) \otimes a_{1}+\cdots+\overline{\mathscr{O}}\left(f_{r}\right) \otimes a_{r}\right)\right|_{C}\right) & =a_{1} \widehat{\operatorname{deg}}\left(\left.\overline{\mathscr{O}}\left(f_{1}\right)\right|_{C}\right)+\cdots+a_{r} \widehat{\operatorname{deg}}\left(\left.\overline{\mathscr{O}}\left(f_{r}\right)\right|_{C}\right) \\
& =\sum_{i=1}^{r} a_{i}\left(\sum_{x \in C(\mathbb{C})} f_{i}(x)\right)=0 .
\end{aligned}
$$

Therefore, $\widehat{\operatorname{deg}}\left(-\left.\right|_{C}\right): \widehat{\operatorname{Pic}}_{C^{0}}(X) \otimes_{\mathbb{Z}} \mathbb{R} \rightarrow \mathbb{R}$ descends to a homomorphism $\widehat{\operatorname{Pic}}_{C^{0}}(X)_{\mathbb{R}}$ $\rightarrow \mathbb{R}$, denoted also by $\widehat{\operatorname{deg}}\left(-\left.\right|_{C}\right)$. Using this homomorphism, we define

$$
\widehat{\operatorname{deg}}\left(-\left.\right|_{C}\right): \widehat{\operatorname{Div}}_{C^{0}}(X)_{\mathbb{R}} \rightarrow \mathbb{R}
$$

to be $\widehat{\operatorname{deg}}\left(\left.\bar{D}\right|_{C}\right):=\widehat{\operatorname{deg}}\left(\left.\overline{\mathscr{O}}_{\mathbb{R}}(\bar{D})\right|_{C}\right)$ for $\bar{D} \in \widehat{\operatorname{Div}}_{C^{0}}(X)_{\mathbb{R}}$. If there are effective Cartier 
divisors $D_{1}, \ldots, D_{l}$ and $a_{1}, \ldots, a_{l} \in \mathbb{R}$ such that $D=a_{1} D_{1}+\cdots+a_{l} D_{l}$ and $C \nsubseteq \operatorname{Supp}\left(D_{i}\right)$ for all $i$, then we can see that

$$
\widehat{\operatorname{deg}}\left(\left.\bar{D}\right|_{C}\right)=\sum_{i=1}^{l} a_{i} \log \#\left(\mathscr{O}_{C}\left(D_{i}\right) / \mathscr{O}_{C}\right)+\frac{1}{2} \sum_{x \in C(\mathbb{C})} g_{\operatorname{can}}(x) .
$$

Let $\mathscr{T}$ be a type for Green functions on $X$ such that $C^{0} \subseteq \mathscr{T}, \mathscr{T}$ is real valued and $-\mathscr{T} \subseteq \mathscr{T}$. Let $\bar{D}=(D, g)$ be an arithmetic $\mathbb{R}$-Cartier divisor of $\mathscr{T}$-type on $X$. There is $h \in \mathscr{T}(X)$ such that $g-h$ is an $F_{\infty}$-invariant $D$-Green function of $C^{0}$-type. We would like to define $\widehat{\operatorname{deg}}\left(\left.\bar{D}\right|_{C}\right)$ to be the following quantity:

$$
\widehat{\operatorname{deg}}\left(\left.(D, g-h)\right|_{C}\right)+\frac{1}{2} \sum_{x \in C(\mathbb{C})} h(x) .
$$

Indeed, it does not depend on the choice of $h$. Let $h^{\prime}$ be another element of $\mathscr{T}(X)$ such that $g-h^{\prime}$ is an $F_{\infty}$-invariant $D$-Green function of $C^{0}$-type. We can find $u \in C^{0}(X)$ such that $g-h=g-h^{\prime}+u$ (a.e.), so that $h^{\prime}=h+u$ over $X(\mathbb{C})$. Therefore,

$$
\begin{aligned}
\widehat{\operatorname{deg}}\left(\left.\left(D, g-h^{\prime}\right)\right|_{C}\right)+\frac{1}{2} & \sum_{x \in C(\mathbb{C})} h^{\prime}(x)=\widehat{\operatorname{deg}}\left(\left.(D,(g-h)-u)\right|_{C}\right)+\frac{1}{2} \sum_{x \in C(\mathbb{C})}(h+u)(x) \\
& =\widehat{\operatorname{deg}}\left(\left.(D,(g-h))\right|_{C}\right)-\frac{1}{2} \sum_{x \in C(\mathbb{C})} u(x)+\frac{1}{2} \sum_{x \in C(\mathbb{C})}(h+u)(x) \\
& =\widehat{\operatorname{deg}}\left(\left.(D, g-h)\right|_{C}\right)+\frac{1}{2} \sum_{x \in C(\mathbb{C})} h(x) .
\end{aligned}
$$

Note that if there are effective Cartier divisors $D_{1}, \ldots, D_{l}$ and $a_{1}, \ldots, a_{l} \in \mathbb{R}$ such that $D=a_{1} D_{1}+\cdots+a_{l} D_{l}$ and $C \nsubseteq \operatorname{Supp}\left(D_{i}\right)$ for all $i$, then

$$
\widehat{\operatorname{deg}}\left(\left.\bar{D}\right|_{C}\right)=\sum_{i=1}^{l} a_{i} \log \#\left(\mathscr{O}_{C}\left(D_{i}\right) / \mathscr{O}_{C}\right)+\frac{1}{2} \sum_{x \in C(\mathbb{C})} g_{\operatorname{can}}(x) .
$$

Moreover, $\widehat{\operatorname{deg}}\left(-\left.\right|_{C}\right): \widehat{\operatorname{Div}}_{\mathscr{T}}(X)_{\mathbb{R}} \rightarrow \mathbb{R}$ is a homomorphism.

Let $Z_{1}(X)$ be the group of 1 -cycles on $X$ and $Z_{1}(X)_{\mathbb{R}}=Z_{1}(X) \otimes_{\mathbb{Z}} \mathbb{R}$. Let $Z$ be an element of $Z_{1}(X)_{\mathbb{R}}$. There is a unique expression $Z=a_{1} C_{1}+\cdots+a_{l} C_{l}$ such that $a_{1}, \ldots, a_{l} \in \mathbb{R}$ and $C_{1}, \ldots, C_{l}$ are 1-dimensional closed integral schemes on $X$. For $\bar{D} \in \widehat{\operatorname{Div}}_{\mathscr{T}}(X)_{\mathbb{R}}$, we define

$$
\widehat{\operatorname{deg}}(\bar{D} \mid Z):=\sum_{i=1}^{l} a_{i} \widehat{\operatorname{deg}}\left(\left.\bar{D}\right|_{C_{i}}\right) .
$$

Note that $\widehat{\operatorname{deg}}(\bar{D} \mid C)=\widehat{\operatorname{deg}}\left(\left.\bar{D}\right|_{C}\right)$ for a 1-dimensional closed integral scheme $C$ on $X$. 


\section{$\S 6$. Positivity of arithmetic $\mathbb{R}$-Cartier divisors}

In this section, we will introduce several kinds of positivity for arithmetic $\mathbb{R}$ Cartier divisors and investigate their properties. Throughout this section, $X$ will be a generically smooth projective and normal arithmetic variety.

\section{$\S 6.1$. Definitions}

Let $\bar{D}=(D, g)$ be an arithmetic $\mathbb{R}$-Cartier divisor on $X$, that is, $D \in \operatorname{Div}(X)_{\mathbb{R}}$ and $g$ is an $F_{\infty}$-invariant locally integrable function on $X(\mathbb{C})$. The ampleness, adequateness, nefness, bigness and pseudo-effectivity of $\bar{D}$ are defined as follows:

- Ample: First we recall the ampleness of a $C^{\infty}$-hermitian invertible sheaf. According to [13], an $F_{\infty}$-invariant $C^{\infty}$-hermitian invertible sheaf $\overline{\mathcal{L}}=(\mathcal{L}, h)$ on $X$ is said to be ample if $\mathcal{L}$ is ample, $c_{1}(\overline{\mathcal{L}})$ is a positive form and $H^{0}\left(X, \mathcal{L}^{\otimes n}\right)$ is generated by elements of

$$
\left\{s \in H^{0}\left(X, \mathcal{L}^{\otimes n}\right) \mid\|s\|_{\text {sup }}<1\right\}
$$

as a $\mathbb{Z}$-module for $n \gg 1$. Note that our definition is slightly stronger than Zhang's [25], where the semipositivity of $c_{1}(\overline{\mathcal{L}})$ is assumed instead of positivity.

We say $\bar{D}$ is ample if there are $a_{1}, \ldots, a_{r} \in \mathbb{R}_{>0}$ and ample arithmetic $\mathbb{Q}$ Cartier divisors $\bar{A}_{1}, \ldots, \bar{A}_{r}$ of $C^{\infty}$-type (i.e., $\overline{\mathscr{O}}\left(n_{i} \bar{A}_{i}\right)$ is an ample $C^{\infty}$-hermitian invertible sheaf for some $n_{i} \in \mathbb{Z}_{>0}$ in the above sense) such that

$$
\bar{D}=a_{1} \bar{A}_{1}+\cdots+a_{r} \bar{A}_{r} .
$$

Note that an ample arithmetic $\mathbb{R}$-Cartier divisor is of $C^{\infty}$-type. The set of all ample arithmetic $\mathbb{R}$-Cartier divisors on $X$ is denoted by $\widehat{\operatorname{Amp}}(X)_{\mathbb{R}}$. By applying [16, Lemma 1.1.3] to the case where $P=\widehat{\operatorname{Div}}_{C^{\infty}}(X)_{\mathbb{Q}}, m=1, b_{1}=0, A={ }^{t}(0, \ldots, 0)$ and $x_{1}=\bar{A}_{1}, \ldots, x_{r}=\bar{A}_{r}$, we can see that

$$
\widehat{\operatorname{Amp}}(X)_{\mathbb{R}} \cap \widehat{\operatorname{Div}}_{C^{\infty}}(X)_{\mathbb{Q}}=\left\{\begin{array}{l|l}
\bar{D} & \begin{array}{l}
\overline{\mathscr{O}}(n \bar{D}) \text { is an ample } C^{\infty} \text {-hermitian } \\
\text { invertible sheaf on } X \text { for some } n \in \mathbb{Z}_{>0}
\end{array}
\end{array}\right\} .
$$

- Adequate: $\bar{D}$ is said to be adequate if there are an ample arithmetic $\mathbb{R}$-Cartier divisor $\bar{A}$ and a non-negative $F_{\infty}$-invariant continuous function $f$ on $X(\mathbb{C})$ such that $\bar{D}=\bar{A}+(0, f)$. Note that an adequate arithmetic $\mathbb{R}$-Cartier divisor is of $C^{0}$-type.

- Nef: We say $\bar{D}$ is nef if the following properties holds:

(a) $\bar{D}$ is of $\mathrm{PSH}_{\mathbb{R}}$-type.

(b) $\widehat{\operatorname{deg}}\left(\left.\bar{D}\right|_{C}\right) \geq 0$ for all 1-dimensional closed integral subschemes $C$ of $X$. 
The cone of all nef arithmetic $\mathbb{R}$-Cartier divisors on $X$ is denoted by $\widehat{\operatorname{Nef}}(X)_{\mathbb{R}}$. Moreover, the cone of all nef arithmetic $\mathbb{R}$-Cartier divisors of $C^{\infty}$-type (resp. $C^{0}$ type) on $X$ is denoted by $\widehat{\operatorname{Nef}}_{C^{\infty}}(X)_{\mathbb{R}}\left(\right.$ resp. $\left.\widehat{\operatorname{Nef}}_{C^{0}}(X)_{\mathbb{R}}\right)$.

- Big: Let us fix a type $\mathscr{T}$ for Green functions. We say $\bar{D}$ is a big arithmetic $\mathbb{R}$-Cartier divisor of $\mathscr{T}$-type if $\bar{D} \in \widehat{\operatorname{Div}}_{\mathscr{T} b}(X)_{\mathbb{R}}$ (i.e. $\bar{D} \in \widehat{\operatorname{Div}}_{\mathscr{T}}(X)_{\mathbb{R}}$ and $g$ is of bounded type) and $\widehat{\operatorname{vol}}(\bar{D})>0$.

- Pseudo-effective: $\bar{D}$ is said to be pseudo-effective if $\bar{D}$ is of $C^{0}$-type and there are arithmetic $\mathbb{R}$-Cartier divisors $\bar{D}_{1}, \ldots, \bar{D}_{r}$ of $C^{0}$-type and sequences $\left\{a_{n 1}\right\}_{n=1}^{\infty}, \ldots,\left\{a_{n r}\right\}_{n=1}^{\infty}$ in $\mathbb{R}$ such that $\lim _{n \rightarrow \infty} a_{n i}=0$ for all $i=1, \ldots, r$ and $\widehat{\operatorname{vol}}\left(\bar{D}+a_{n 1} \bar{D}_{1}+\cdots+a_{n r} \bar{D}_{r}\right)>0$ for all $n \gg 1$.

\section{$\S 6.2$. Properties of ample arithmetic $\mathbb{R}$-Cartier divisors}

In this subsection, we consider several properties of ample arithmetic $\mathbb{R}$-Cartier divisors. Let us begin with the following proposition.

Proposition 6.2.1. (1) If $\bar{A}$ and $\bar{B}$ are ample (resp. adequate) arithmetic $\mathbb{R}$ Cartier divisors and $a \in \mathbb{R}_{>0}$, then $\bar{A}+\bar{B}$ and $a \bar{A}$ are also ample (resp. adequate).

(2) If $\bar{A}$ is an ample arithmetic $\mathbb{R}$-Cartier divisor, then there are an ample arithmetic $\mathbb{Q}$-Cartier divisor $\bar{A}^{\prime}$ and an ample arithmetic $\mathbb{R}$-Cartier divisor $\bar{A}^{\prime \prime}$ such that $\bar{A}=\bar{A}^{\prime}+\bar{A}^{\prime \prime}$.

(3) Let $\bar{A}$ be an ample (resp. adequate) arithmetic $\mathbb{R}$-Cartier divisor and let $\bar{L}_{1}$, $\ldots, \bar{L}_{n}$ be arithmetic $\mathbb{R}$-Cartier divisors of $C^{\infty}$-type (resp. of $C^{0}$-type). Then there is $\delta \in \mathbb{R}_{>0}$ such that $\bar{A}+\delta_{1} \bar{L}_{1}+\cdots+\delta_{n} \bar{L}_{n}$ is ample (resp. adequate) for $\delta_{1}, \ldots, \delta_{n} \in \mathbb{R}$ with $\left|\delta_{1}\right|+\cdots+\left|\delta_{n}\right| \leq \delta$.

(4) If $\bar{A}$ is an adequate arithmetic $\mathbb{R}$-Cartier divisor, then $\widehat{\operatorname{vol}}(\bar{A})>0$.

Proof. (1) and (2) are obvious.

(3) First we assume that $\bar{A}$ is ample and that $\bar{L}_{1}, \ldots, \bar{L}_{n}$ are of $C^{\infty}$-type. We set $\bar{L}_{i}=\sum_{j=1}^{l} b_{i j} \bar{M}_{j}$ such that $\bar{M}_{1}, \ldots, \bar{M}_{l}$ are arithmetic $\mathbb{Q}$-Cartier divisors of $C^{\infty}$-type and $b_{i j} \in \mathbb{R}$. Then, as

$$
\bar{A}+\sum_{i=1}^{n} \delta_{i} \bar{L}_{i}=\bar{A}+\sum_{j=1}^{l}\left(\sum_{i=1}^{n} \delta_{i} b_{i j}\right) \bar{M}_{j}
$$

we may assume that $\bar{L}_{1}, \ldots, \bar{L}_{n}$ are arithmetic $\mathbb{Q}$-Cartier divisors of $C^{\infty}$-type. Moreover, by (1) and (2), we may further assume that $\bar{A}$ is an ample arithmetic $\mathbb{Q}$-Cartier divisor. 
Let us choose $\delta \in \mathbb{Q}_{>0}$ such that $\bar{A} \pm \delta \bar{L}_{i}$ is ample for every $i=1, \ldots, n$. Note that

$$
\sum_{i=1}^{n} \frac{\left|\delta_{i}\right|}{\delta}\left(\bar{A}+\operatorname{sign}\left(\delta_{i}\right) \delta \bar{L}_{i}\right)=\left(\sum_{i=1}^{n} \frac{\left|\delta_{i}\right|}{\delta}\right) \bar{A}+\sum_{i=1}^{n} \delta_{i} \bar{L}_{i}
$$

where $\operatorname{sign}(a)$ for $a \in \mathbb{R}$ is given by

$$
\operatorname{sign}(a)= \begin{cases}1 & \text { if } a \geq 0 \\ -1 & \text { if } a<0\end{cases}
$$

Hence, if $\sum_{i=1}\left|\delta_{i}\right| \leq \delta$, then $\bar{A}+\sum_{i=1}^{n} \delta_{i} \bar{L}_{i}$ is ample.

Next we assume that $\bar{A}$ is adequate and that $\bar{L}_{1}, \ldots, \bar{L}_{n}$ are of $C^{0}$-type. Then there are an ample arithmetic $\mathbb{R}$-Cartier divisor $\bar{A}^{\prime}$ and $u \in C^{0}(X)$ such that $u \geq 0$ and $\bar{A}=\bar{A}^{\prime}+(0, u)$. As $\bar{A}^{\prime}-(0, \epsilon)$ is ample for $0<\epsilon \ll 1$ by the previous observation, we may assume that $u \geq \epsilon$ for some positive number $\epsilon$. By virtue of the Stone-Weierstrass theorem, we can find $v_{1}, \ldots, v_{n} \in C^{0}(X)$ such that $v_{i} \geq 0(\forall i)$, $\epsilon \geq v_{1}+\cdots+v_{n}$ and $\bar{L}_{i}^{\prime}:=\bar{L}_{i}+\left(0, v_{i}\right)$ is of $C^{\infty}$-type for all $i$. By the previous case, we can find $0<\delta<1$ such that $\bar{A}^{\prime}+\delta_{1} \bar{L}_{1}^{\prime}+\cdots+\delta_{n} \bar{L}_{n}^{\prime}$ is ample for $\delta_{1}, \ldots, \delta_{n} \in \mathbb{R}$ with $\left|\delta_{1}\right|+\cdots+\left|\delta_{n}\right| \leq \delta$. Note that

$$
\bar{A}+\delta_{1} \bar{L}_{1}+\cdots+\delta_{n} \bar{L}_{n}=\bar{A}^{\prime}+\delta_{1} \bar{L}_{1}^{\prime}+\cdots+\delta_{n} \bar{L}_{n}^{\prime}+\left(0, u-\delta_{1} v_{1}-\cdots-\delta_{n} v_{n}\right)
$$

and

$$
u-\delta_{1} v_{1}-\cdots-\delta_{n} v_{n} \geq u-v_{1}-\cdots-v_{n} \geq 0
$$

as required.

(4) Clearly we may assume that $\bar{A}$ is ample, so that the assertion follows from (2) and (4) of Theorem 5.2.2.

Next we prove the following.

Proposition 6.2.2. (1) If $\bar{A}$ is an ample arithmetic $\mathbb{R}$-Cartier divisor and $\bar{B}$ is a nef arithmetic $\mathbb{R}$-Cartier divisor of $C^{\infty}$-type, then $\bar{A}+\bar{B}$ is ample.

(2) If $\bar{A}$ is an adequate arithmetic $\mathbb{R}$-Cartier divisor and $\bar{B}$ is a nef arithmetic $\mathbb{R}$-Cartier divisor of $C^{0}$-type, then $\bar{A}+\bar{B}$ is adequate.

Proof. (1) We set $\bar{B}=b_{1} \bar{B}_{1}+\cdots+b_{n} \bar{B}_{n}$ where $b_{1}, \ldots, b_{n} \in \mathbb{R}$ and $\bar{B}_{1}, \ldots, \bar{B}_{n}$ are arithmetic $\mathbb{Q}$-Cartier divisors of $C^{\infty}$-type. We choose an ample arithmetic $\mathbb{Q}$-Cartier divisor $\bar{A}_{1}$ and an ample arithmetic $\mathbb{R}$-Cartier divisor $\bar{A}_{2}$ such that $\bar{A}=\bar{A}_{1}+\bar{A}_{2}$. Then, by Proposition $6.2 .1(3)$, there are $\delta_{1}, \ldots, \delta_{n} \in \mathbb{R}_{>0}$ such that

$$
\bar{A}_{1}+\sum_{i=1}^{n} \delta_{i} \bar{B}_{i} \text { and } \bar{A}_{2}-\sum_{i=1}^{n} \delta_{i} \bar{B}_{i}
$$


are ample and $b_{i}+\delta_{i} \in \mathbb{Q}$ for all $i$. Moreover, we can take an ample arithmetic $\mathbb{Q}$-Cartier divisor $\bar{A}_{3}$ and an ample arithmetic $\mathbb{R}$-Cartier divisor $\bar{A}_{4}$ such that

$$
\bar{A}_{2}-\sum_{i=1}^{n} \delta_{i} \bar{B}_{i}=\bar{A}_{3}+\bar{A}_{4} .
$$

Then, since

$$
\bar{A}_{1}+\sum_{i=1}^{n} \delta_{i} \bar{B}_{i}+\bar{B}=\bar{A}_{1}+\sum_{i=1}^{n}\left(b_{i}+\delta_{i}\right) \bar{B}_{i}
$$

is a nef arithmetic $\mathbb{Q}$-Cartier divisor of $C^{\infty}$-type, $\bar{A}_{3}+\bar{A}_{1}+\sum_{i=1}^{n} \delta_{i} \bar{B}_{i}+\bar{B}$ is an ample arithmetic $\mathbb{Q}$-Cartier divisor by [14, Lemma 5.6]. Therefore,

$$
\bar{A}+\bar{B}=\bar{A}_{4}+\bar{A}_{3}+\bar{A}_{1}+\sum_{i=1}^{n} \delta_{i} \bar{B}_{i}+\bar{B}
$$

is an ample arithmetic $\mathbb{R}$-Cartier divisor.

(2) Clearly we may assume that $\bar{A}$ is ample. By Proposition 6.2.1(3), there is a positive real number $\delta$ such that $\frac{1}{2} \bar{A}-(0, \delta)$ is ample. Note that $\frac{1}{2} A+B$ is ample, that is, $\frac{1}{2} A+B$ is a linear combination of ample Cartier divisors with positive coefficients, which can be checked in the same way as above. Thus, by Theorem 4.6(2), there is $u \in C^{0}(X)$ (i.e., $u$ is an $F_{\infty}$-invariant continuous function in $X(\mathbb{C})$ ) such that $0 \leq u<\delta$ on $X(\mathbb{C})$ and $\frac{1}{2} \bar{A}+\bar{B}+(0, u)$ is a nef $\mathbb{R}$-Cartier divisor of $C^{\infty}$-type. Then, by (1),

$$
\frac{1}{2} \bar{A}-(0, \delta)+\frac{1}{2} \bar{A}+\bar{B}+(0, u)
$$

is ample. Thus

$$
\bar{A}+\bar{B}=\frac{1}{2} \bar{A}-(0, \delta)+\frac{1}{2} \bar{A}+\bar{B}+(0, u)+(0, \delta-u)
$$

is adequate.

Finally let us record the following lemma.

Lemma 6.2.3. Let $\bar{D}_{1}=\left(D_{1}, g_{1}\right)$ and $\bar{D}_{2}=\left(D_{2}, g_{2}\right)$ be arithmetic $\mathbb{R}$-Cartier divisors of $\mathrm{PSH}_{\mathbb{R}}$-type on $X$. If $D_{1}=D_{2}, g_{1} \leq g_{2}$ (a.e.) and $\bar{D}_{1}$ is nef, then $\bar{D}_{2}$ is also nef.

Proof. Since $D_{1}=D_{2}$, there is a $\phi \in\left(\mathrm{PSH}_{\mathbb{R}}-\mathrm{PSH}_{\mathbb{R}}\right)(X(\mathbb{C}))$ such that $g_{2}=$ $g_{1}+\phi$ (a.e.) and $\phi \geq 0$ (a.e.). Note that $\phi \geq 0$ by Lemma 2.3.1. Let $C$ be a 1-dimensional closed integral subscheme of $X$. Then

$$
\widehat{\operatorname{deg}}\left(\left.\bar{D}_{2}\right|_{C}\right)=\operatorname{deg}\left(\left.\bar{D}_{1}\right|_{C}\right)+\frac{1}{2} \sum_{y \in C(\mathbb{C})} \phi(y) \geq \operatorname{deg}\left(\left.\bar{D}_{1}\right|_{C}\right) \geq 0 .
$$




\section{§6.3. Criteria of bigness and pseudo-effectivity}

The purpose of this subsection is to prove the following propositions.

Proposition 6.3.1. For $\bar{D}=(D, g) \in \widehat{\operatorname{Div}}_{C^{0}}(X)_{\mathbb{R}}$, the following are equivalent:

(1) $\bar{D}$ is big, that is, $\widehat{\operatorname{vol}}(\bar{D})>0$.

(2) For any $\bar{A} \in \widehat{\operatorname{Div}}_{C^{0}}(X)_{\mathbb{R}}$, there are a positive integer $n$ and a non-zero rational function $\phi$ such that $\bar{A} \leq n \bar{D}+\widehat{(\phi)}$.

Proof. " $(2) \Rightarrow(1) "$ is obvious.

Let us consider " $(1) \Rightarrow(2)$ ". By using Lemmas 5.2.3 and 5.2.4, we can find effective arithmetic $\mathbb{R}$-Cartier divisors $\bar{A}^{\prime}$ and $\bar{A}^{\prime \prime}$ of $C^{0}$-type such that $\bar{A}=\bar{A}^{\prime}-\bar{A}^{\prime \prime}$. Note that $\bar{A} \leq \bar{A}^{\prime}$. Thus we may assume $\bar{A}$ is effective. By the continuity of $\widehat{\text { vol }}$ (cf. Theorem 5.2.2), there is a positive integer $m$ such that

$$
\widehat{\operatorname{vol}}(\bar{D}-(1 / m) \bar{A})>0,
$$

that is, $\widehat{\operatorname{vol}}(m \bar{D}-\bar{A})>0$, so that there is a positive integer $n$ and a non-zero rational function $\phi$ such that

$$
n(m \bar{D}-\bar{A})+\widehat{(\phi)} \geq 0 .
$$

Thus $m n \bar{D}+\widehat{(\phi)} \geq n \bar{A} \geq \bar{A}$.

Proposition 6.3.2. For $\bar{D}=(D, g) \in \widehat{\operatorname{Div}}_{C^{0}}(X)_{\mathbb{R}}$, the following are equivalent:

(1) $\bar{D}$ is pseudo-effective.

(2) For any big arithmetic $\mathbb{R}$-Cartier divisor $\bar{A}$ of $C^{0}$-type, $\widehat{\operatorname{vol}}(\bar{D}+\bar{A})>0$.

(3) There is a big arithmetic $\mathbb{R}$-Cartier divisor $\bar{A}$ of $C^{0}$-type such that $\widehat{\operatorname{vol}}(\bar{D}+$ $(1 / n) \bar{A})>0$ for all $n \geq 1$.

Proof. It is sufficient to see that (1) implies (2). As $\bar{D}$ is pseudo-effective, there are arithmetic $\mathbb{R}$-Cartier divisors $\bar{D}_{1}, \ldots, \bar{D}_{r}$ of $C^{0}$-type and sequences $\left\{a_{m 1}\right\}_{m=1}^{\infty}, \ldots$, $\left\{a_{m r}\right\}_{m=1}^{\infty}$ in $\mathbb{R}$ such that $\lim _{m \rightarrow \infty} a_{m i}=0$ for all $i=1, \ldots, r$ and $\widehat{\operatorname{vol}}\left(\bar{D}+a_{m 1} \bar{D}_{1}+\right.$ $\left.\cdots+a_{m r} \bar{D}_{r}\right)>0$ for all $m \gg 1$. By the continuity of $\widehat{\text { vol, }}$, there is a sufficiently large positive integer $m$ such that $\bar{A}-\left(a_{m 1} \bar{D}_{1}+\cdots+a_{m r} \bar{D}_{r}\right)$ is big. Thus

$$
\widehat{\operatorname{vol}}(\bar{D}+\bar{A}) \geq \widehat{\operatorname{vol}}\left(\bar{D}+a_{m 1} \bar{D}_{1}+\cdots+a_{m r} \bar{D}_{r}\right)>0 .
$$

Proposition 6.3.3. If $\bar{D}=(D, g)$ is a pseudo-effective arithmetic $\mathbb{R}$-Cartier divisor of $C^{0}$-type such that $D$ is big on the generic fiber $X_{\mathbb{Q}}$ (i.e., $\operatorname{vol}\left(D_{\mathbb{Q}}\right)>0$ on $\left.X_{\mathbb{Q}}\right)$, then $\bar{D}+(0, \epsilon)$ is big for all $\epsilon \in \mathbb{R}_{>0}$. 
Proof. Let $\bar{A}$ be an ample arithmetic Cartier divisor on $X$. Since $D$ is big on $X_{\mathbb{Q}}$, by using the continuity of the volume function over $X_{\mathbb{Q}}$ (cf. [10, I, Corollary 2.2.45]), we can see that there are a positive integer $m$ and a non-zero rational function $\phi$ such that

$$
m D-A+(\phi) \geq 0 .
$$

If we set $(L, h)=m \bar{D}-\bar{A}+\widehat{(\phi)}$, then $h$ is an $L$-Green function of $C^{0}$-type and $L$ is effective. Thus there is a positive number $\lambda$ such that

$$
m \bar{D}-\bar{A}+\widehat{(\phi)} \geq(0,-\lambda)
$$

that is, $m \bar{D}+(0, \lambda) \geq \bar{A}-\widehat{(\phi)}$. We choose a sufficiently large positive integer $n$ such that

$$
\frac{\lambda}{n+m} \leq \epsilon
$$

Then

$$
\begin{aligned}
\bar{D}+\frac{1}{n}(\bar{A}-\widehat{(\phi)}) & \leq \bar{D}+\frac{1}{n}(m \bar{D}+(0, \lambda))=\left(1+\frac{m}{n}\right)\left(\bar{D}+\left(0, \frac{\lambda}{n+m}\right)\right) \\
& \leq\left(1+\frac{m}{n}\right)(\bar{D}+(0, \epsilon)) .
\end{aligned}
$$

Note that $\bar{A}-\widehat{(\phi)}$ is ample, so that $\bar{D}+(1 / n)(\bar{A}-\widehat{(\phi)})$ is big by Proposition 6.3.2, and hence $\bar{D}+(0, \epsilon)$ is also big.

Remark 6.3.4. It is very natural to ask whether $\hat{H}^{0}(X, n(\bar{D}+(0, \epsilon))) \neq\{0\}$ for some $n \in \mathbb{Z}_{>0}$ in the case where $D$ is not necessarily big on $X_{\mathbb{Q}}$. This does not hold in general. For example, let $\mathbb{P}_{\mathbb{Z}}^{1}=\operatorname{Proj}\left(\mathbb{Z}\left[T_{0}, T_{1}\right]\right)$ be the projective line over $\mathbb{Z}$ and $\bar{D}=a\left(\widehat{T_{1} / T_{0}}\right)$ for $a \in \mathbb{R} \backslash \mathbb{Q}$. It is easy to see that $\bar{D}$ is pseudo-effective and $H^{0}\left(\mathbb{P}_{\mathbb{Z}}^{1}, n D\right)=\{0\}$ for all $n \in \mathbb{Z}_{>0}$. Thus $\hat{H}^{0}\left(\mathbb{P}_{\mathbb{Z}}^{1}, n(\bar{D}+(0, \epsilon))=\{0\}\right.$ for $\epsilon \in \mathbb{R}_{>0}$ and $n \in \mathbb{Z}_{>0}$.

\section{$\S 6.4$. Intersection number of arithmetic $\mathbb{R}$-Cartier divisors of $C^{0}$-type}

Let

$$
\widehat{\operatorname{Div}}_{C} \infty(X) \times \cdots \times \widehat{\operatorname{Div}}_{C} \infty(X) \rightarrow \mathbb{R}
$$

be a symmetric multi-linear map over $\mathbb{Z}$ given by

$$
\left(\bar{D}_{1}, \ldots, \bar{D}_{d}\right) \mapsto \widehat{\operatorname{deg}}\left(\bar{D}_{1} \cdots \bar{D}_{d}\right):=\widehat{\operatorname{deg}}\left(\widehat{c}_{1}\left(\overline{\mathscr{O}}\left(\bar{D}_{1}\right)\right) \cdots \widehat{c}_{1}\left(\overline{\mathscr{O}}\left(\bar{D}_{d}\right)\right)\right)
$$

which extends to a symmetric multi-linear map

$$
\left(\widehat{\operatorname{Div}}_{C^{\infty}}(X) \otimes_{\mathbb{Z}} \mathbb{R}\right) \times \cdots \times\left(\widehat{\operatorname{Div}}_{C^{\infty}}(X) \otimes_{\mathbb{Z}} \mathbb{R}\right) \rightarrow \mathbb{R}
$$

over $\mathbb{R}$. 
Proposition-Definition 6.4.1. The above multi-linear map

$$
\left(\widehat{\operatorname{Div}}_{C^{\infty}}(X) \otimes_{\mathbb{Z}} \mathbb{R}\right) \times \cdots \times\left(\widehat{\operatorname{Div}}_{C^{\infty}}(X) \otimes_{\mathbb{Z}} \mathbb{R}\right) \rightarrow \mathbb{R}
$$

descends to a symmetric multi-linear map

$$
\widehat{\operatorname{Div}}_{C \infty}(X)_{\mathbb{R}} \times \cdots \times \widehat{\operatorname{Div}}_{C \infty}(X)_{\mathbb{R}} \rightarrow \mathbb{R}
$$

over $\mathbb{R}$, whose value at $\left(\bar{D}_{1}, \ldots, \bar{D}_{d}\right) \in \widehat{\operatorname{Div}}_{C^{\infty}}(X)_{\mathbb{R}} \times \cdots \times \widehat{\operatorname{Div}}_{C^{\infty}}(X)_{\mathbb{R}}$ is also denoted by $\widehat{\operatorname{deg}}\left(\bar{D}_{1} \cdots \bar{D}_{d}\right)$ by abuse of notation.

Proof. Let $a_{1}, \ldots, a_{l} \in \mathbb{R}$ and $\phi_{1}, \ldots, \phi_{l} \in C^{\infty}(X)$ be such that $a_{1} \phi_{1}+\cdots+a_{l} \phi_{l}$ $=0$. By Lemma 5.2.5, it is sufficient to show that

$$
\widehat{\operatorname{deg}}\left(\left(\left(0, \phi_{1}\right) \otimes a_{1}+\cdots+\left(0, \phi_{l}\right) \otimes a_{l}\right) \cdot \bar{D}_{2} \cdots \bar{D}_{d}\right)=0
$$

for all $\bar{D}_{2}, \ldots, \bar{D}_{d} \in \widehat{\operatorname{Div}}_{C}(X)$. First, note that there are 1-dimensional closed integral subschemes $C_{1}, \ldots, C_{r}, c_{1}, \ldots, c_{r} \in \mathbb{Z}$ and a current $T$ of $(d-2, d-2)$ type such that

$$
\bar{D}_{2} \cdots \bar{D}_{d} \sim\left(c_{1} C_{1}+\cdots+c_{r} C_{r}, T\right) .
$$

Then

$$
\begin{aligned}
\widehat{\operatorname{deg}}\left(\left(\left(0, \phi_{1}\right) \otimes a_{1}+\cdots+\left(0, \phi_{l}\right) \otimes a_{l}\right) \cdot \bar{D}_{2} \cdots \bar{D}_{d}\right) \\
=\sum_{i=1}^{l} a_{i} \widehat{\operatorname{deg}}\left(\left(0, \phi_{i}\right) \cdot\left(c_{1} C_{1}+\cdots+c_{r} C_{r}, T\right)\right) \\
=\sum_{i=1}^{l} a_{i}\left(\sum_{j=1}^{r} c_{j} \sum_{y \in C_{j}(\mathbb{C})} \phi_{i}(y)+(1 / 2) \int_{X(\mathbb{C})} d d^{c}\left(\phi_{i}\right) \wedge T\right) \\
=\sum_{j=1}^{r} c_{j} \sum_{y \in C_{j}(\mathbb{C})} \sum_{i=1}^{l} a_{i} \phi_{i}(y)+(1 / 2) \int_{X(\mathbb{C})} d d^{c}\left(\sum_{i=1}^{l} a_{i} \phi_{i}\right) \wedge T=0,
\end{aligned}
$$

as required.

Let $\widehat{\operatorname{Div}}_{C^{0}}^{\text {Nef }}(X)_{\mathbb{R}}$ be the subspace of $\widehat{\operatorname{Div}}_{C^{0}}(X)_{\mathbb{R}}$ generated by $\widehat{\operatorname{Nef}}_{C^{0}}(X)_{\mathbb{R}}$. The purpose of this subsection is to show the following proposition, which gives another construction of the intersection number due to [25, Lemma 6.5], [26, Section 1] and [11, Section 5] (cf. Remark 6.4.3).

Proposition 6.4.2. (1)

$$
\widehat{\operatorname{Div}}_{C^{0} \cap \mathrm{PSH}+C^{\infty}}(X)_{\mathbb{R}} \subseteq \widehat{\operatorname{Div}}_{C^{0}}^{\mathrm{Nef}}(X)_{\mathbb{R}} \subseteq \widehat{\operatorname{Div}}_{C^{0} \cap \mathrm{PSH}-C^{0} \cap \mathrm{PSH}}(X)_{\mathbb{R}} .
$$


(2) The symmetric multi-linear map $\widehat{\operatorname{Div}}_{C}(X)_{\mathbb{R}} \times \cdots \times \widehat{\operatorname{Div}}_{C^{\infty}}(X)_{\mathbb{R}} \rightarrow \mathbb{R}$ given in Proposition-Definition 6.4 .1 extends to a unique symmetric multi-linear map

$$
\widehat{\operatorname{Div}}_{C^{0}}^{\mathrm{Nef}}(X)_{\mathbb{R}} \times \cdots \times \widehat{\operatorname{Div}}_{C^{0}}^{\mathrm{Nef}}(X)_{\mathbb{R}} \rightarrow \mathbb{R}
$$

such that $(\bar{D}, \ldots, \bar{D}) \mapsto \widehat{\operatorname{vol}}(\bar{D})$ for $\bar{D} \in \widehat{\operatorname{Nef}}_{C^{0}}(X)_{\mathbb{R}}$. By abuse of notation, for

$$
\left(\bar{D}_{1}, \ldots, \bar{D}_{d}\right) \in \widehat{\operatorname{Div}}_{C^{0}}^{\mathrm{Nef}}(X)_{\mathbb{R}} \times \cdots \times \widehat{\operatorname{Div}}_{C^{0}}^{\mathrm{Nef}}(X)_{\mathbb{R}},
$$

the image of $\left(\bar{D}_{1}, \ldots, \bar{D}_{d}\right)$ under the above extension is also denoted by

$$
\widehat{\operatorname{deg}}\left(\bar{D}_{1} \cdots \bar{D}_{d}\right) .
$$

Proof. (1) It is obvious that

$$
\widehat{\operatorname{Div}}_{C^{0}}^{\mathrm{Nef}}(X)_{\mathbb{R}} \subseteq \widehat{\operatorname{Div}}_{C^{0} \cap \mathrm{PSH}-C^{0} \cap \mathrm{PSH}}(X)_{\mathbb{R}} .
$$

Let $\bar{D} \in \widehat{\operatorname{Div}}_{C^{0} \cap \mathrm{PSH}+C^{\infty}}(X)_{\mathbb{R}}$. By Proposition 2.3.7, there is an ample arithmetic Cartier divisor $\bar{A}$ with $\bar{D}+\bar{A} \in \widehat{\operatorname{Div}}_{C^{0} \cap \mathrm{PSH}}(X)_{\mathbb{R}}$. Thus it is sufficient to show the following claim:

Claim 6.4.2.1. For $\bar{D} \in \widehat{\operatorname{Div}}_{C^{0} \cap \mathrm{PSH}}(X)_{\mathbb{R}}$, there is an ample arithmetic Cartier divisor $\bar{B}$ such that $\bar{D}+\bar{B} \in \widehat{\operatorname{Nef}}_{C^{0}}(X)_{\mathbb{R}}$ and $D+B$ is ample.

Proof. By the Stone-Weierstrass theorem, there is an $F_{\infty}$-invariant non-negative continuous function $u$ on $X(\mathbb{C})$ such that $\bar{D}-(0, u) \in \widehat{\operatorname{Div}}_{C^{\infty}}(X)_{\mathbb{R}}$. Thus, by Proposition 6.2.1, we can find an ample arithmetic Cartier divisor $\bar{B}$ such that $\bar{D}-(0, u)+\bar{B}$ is ample. In particular, $\bar{D}+\bar{B} \in \widehat{\mathrm{Nef}}_{C^{0}}(X)_{\mathbb{R}}$ and $D+B$ is ample.

(2) Let us begin with the following claim.

Claim 6.4.2.2. (a) For $\bar{D} \in \widehat{\operatorname{Nef}}_{C}(X)_{\mathbb{R}}, \widehat{\operatorname{deg}}\left(\bar{D}^{d}\right)=\widehat{\operatorname{vol}}(\bar{D})$.

(b) $d ! X_{1} \cdots X_{d}=\sum_{\emptyset \neq I \subseteq\{1, \ldots, d\}}(-1)^{d-\#(I)}\left(\sum_{i \in I} X_{i}\right)^{d}$ in $\mathbb{Z}\left[X_{1}, \ldots, X_{d}\right]$.

(c) For $\bar{D}_{1}, \ldots, \bar{D}_{d} \in \widehat{\operatorname{Nef}}_{C^{\infty}}(X)_{\mathbb{R}}$,

$$
\widehat{\operatorname{deg}}\left(\bar{D}_{1} \cdots \bar{D}_{d}\right)=\frac{1}{d !} \sum_{\emptyset \neq I \subseteq\{1, \ldots, d\}}(-1)^{d-\#(I)} \widehat{\operatorname{vol}}\left(\sum_{i \in I} \bar{D}_{i}\right) .
$$

Proof. (a) First we assume that $\bar{D}$ is ample. We set $\bar{D}=a_{1} \bar{A}_{1}+\cdots+a_{l} \bar{A}_{l}$ such that $a_{1}, \ldots, a_{l} \in \mathbb{R}_{>0}$ and $\bar{A}_{i}$ 's are ample arithmetic Cartier divisors. Let us choose sufficiently small positive numbers $\delta_{1}, \ldots, \delta_{l}$ such that $a_{i}+\delta_{i} \in \mathbb{Q}$ for all $i$. Then, 
by [14, Corollary 5.5],

$$
\widehat{\operatorname{deg}}\left(\left(\left(a_{1}+\delta_{1}\right) \bar{A}_{1}+\cdots+\left(a_{l}+\delta_{l}\right) \bar{A}_{l}\right)^{d}\right)=\widehat{\operatorname{vol}}\left(\left(a_{1}+\delta_{1}\right) \bar{A}_{1}+\cdots+\left(a_{l}+\delta_{l}\right) \bar{A}_{l}\right),
$$

and using the continuity of $\widehat{\mathrm{vol}}$, the assertion follows.

Next we consider the general case. Let $\bar{A}$ be an ample arithmetic Cartier divisor of $C^{\infty}$-type. Then, by Proposition $6.2 .2, \bar{D}+\epsilon \bar{A}$ is ample for all $\epsilon>0$. Thus the assertion follows from the previous observation and the continuity of $\widehat{\text { vol. }}$.

(b) In general, let us show that

$$
\sum_{\emptyset \neq I \subseteq\{1, \ldots, d\}}(-1)^{\#(I)}\left(\sum_{i \in I} X_{i}\right)^{l}= \begin{cases}0 & \text { if } l<d, \\ (-1)^{d} d ! X_{1} \cdots X_{d} & \text { if } l=d\end{cases}
$$

for integers $d$ and $l$ with $1 \leq l \leq d$; call this assertion $A(d, l)$. Then $A(1,1)$ is obvious. Moreover, it is easy to see $A(d, 1)$. Note that

$$
\begin{aligned}
\int_{0}^{X_{d}} & \left(\sum_{\emptyset \neq I \subseteq\{1, \ldots, d\}}(-1)^{\#(I)}\left(\sum_{i \in I} X_{i}\right)^{l-1}\right) d X_{d} \\
& =\frac{1}{l} \sum_{\emptyset \neq I \subseteq\{1, \ldots, d\}}(-1)^{\#(I)}\left(\sum_{i \in I} X_{i}\right)^{l}+X_{d} \sum_{\emptyset \neq J \subseteq\{1, \ldots, d-1\}}(-1)^{\#(J)}\left(\sum_{j \in J} X_{j}\right)^{l-1},
\end{aligned}
$$

which shows that $A(d-1, l-1)$ and $A(d, l-1)$ imply $A(d, l)$. Thus (b) follows by double induction on $d$ and $l$.

(c) follows from (a) and (b).

The uniqueness of the symmetric multi-linear map follows from (b) of the previous claim. We set

$$
\widehat{P}=\left\{\bar{D} \in \widehat{\operatorname{Nef}}_{C^{0}}(X)_{\mathbb{R}} \mid D \text { is ample }\right\} .
$$

Note that $\bar{D}+\bar{A} \in \widehat{P}$ for all $\bar{D} \in \widehat{\operatorname{Nef}}_{C^{0}}(X)_{\mathbb{R}}$ and $\bar{A} \in \widehat{\operatorname{Amp}}(X)_{\mathbb{R}}$. In particular,

$$
\widehat{\operatorname{Div}}_{C^{0}}^{\mathrm{Nef}}(X)_{\mathbb{R}}=\left\{\bar{D}-\bar{D}^{\prime} \mid \bar{D}, \bar{D}^{\prime} \in \widehat{P}\right\} .
$$

For $\left(\bar{D}_{1}, \ldots, \bar{D}_{d}\right) \in \widehat{P} \times \cdots \times \widehat{P}$, we define

$$
\alpha\left(\bar{D}_{1}, \ldots, \bar{D}_{d}\right):=\frac{1}{d !} \sum_{\emptyset \neq I \subseteq\{1, \ldots, d\}}(-1)^{d-\#(I)} \widehat{\operatorname{vol}}\left(\sum_{i \in I} \bar{D}_{i}\right) .
$$

Claim 6.4.2.4. $\alpha$ is symmetric and

$$
\alpha\left(a \bar{D}_{1}+b \bar{D}_{1}^{\prime}, \bar{D}_{2}, \ldots, \bar{D}_{d}\right)=a \alpha\left(\bar{D}_{1}, \bar{D}_{2}, \ldots, \bar{D}_{d}\right)+b \alpha\left(\bar{D}_{1}^{\prime}, \bar{D}_{2}, \ldots, \bar{D}_{d}\right)
$$

for $\bar{D}_{1}, \bar{D}_{1}^{\prime}, \bar{D}_{2}, \ldots, \bar{D}_{d} \in \widehat{P}$ and $a, b \in \mathbb{R}_{\geq 0}$ with $a+b>0$. 
Proof. Clearly $\alpha$ is symmetric. By Theorem 4.6, for any $\epsilon>0$, there are nonnegative $F_{\infty}$-invariant continuous functions $u_{1}, u_{1}^{\prime}, u_{2}, \ldots, u_{d}$ such that

$$
\left\|u_{1}\right\|_{\text {sup }} \leq \epsilon, \quad\left\|u_{1}^{\prime}\right\|_{\text {sup }} \leq \epsilon, \quad\left\|u_{2}\right\|_{\text {sup }} \leq \epsilon, \ldots,\left\|u_{d}\right\|_{\text {sup }} \leq \epsilon
$$

and $\bar{D}_{1}(\epsilon):=\bar{D}_{1}+\left(0, u_{1}\right), \bar{D}_{1}^{\prime}(\epsilon):=\bar{D}_{1}^{\prime}+\left(0, u_{1}^{\prime}\right), \bar{D}_{2}(\epsilon):=\bar{D}_{2}+\left(0, u_{2}\right), \ldots$, $\bar{D}_{d}(\epsilon):=\bar{D}_{d}+\left(0, u_{d}\right)$ are elements of $\widehat{\operatorname{Nef}}_{C}(X)_{\mathbb{R}}$. Then, by Claim 6.4.2.2,

$$
\begin{aligned}
\alpha\left(a \bar{D}_{1}(\epsilon)+\right. & \left.b \bar{D}_{1}^{\prime}(\epsilon), \bar{D}_{2}(\epsilon), \ldots, \bar{D}_{d}(\epsilon)\right) \\
& =a \alpha\left(\bar{D}_{1}(\epsilon), \bar{D}_{2}(\epsilon), \ldots, \bar{D}_{d}(\epsilon)\right)+b \alpha\left(\bar{D}_{1}^{\prime}(\epsilon), \bar{D}_{2}(\epsilon), \ldots, \bar{D}_{d}(\epsilon)\right) .
\end{aligned}
$$

Thus, using the continuity of $\widehat{\text { vol }}$, we obtain the assertion of the claim.

By the above claim together with Lemma 6.4.4 below, we obtain the existence of the symmetric multi-linear map. Finally we need to check

$$
\widehat{\operatorname{vol}}(\bar{D})=\widehat{\operatorname{deg}}\left(\bar{D}^{d}\right)
$$

for $\bar{D} \in \widehat{\operatorname{Nef}}_{C^{0}}(X)_{\mathbb{R}}$. Let $\bar{A}$ be an ample arithmetic $\mathbb{R}$-Cartier divisor of $C^{\infty}$-type. As $\bar{D}+\epsilon \bar{A} \in \widehat{P}$ for $\epsilon>0$, we have

$$
\widehat{\operatorname{vol}}(\bar{D}+\epsilon \bar{A})=\widehat{\operatorname{deg}}\left((\bar{D}+\epsilon \bar{A})^{d}\right)=\sum_{i=0}^{d}\left(\begin{array}{l}
d \\
i
\end{array}\right) \epsilon^{i} \widehat{\operatorname{deg}}\left(\bar{D}^{d-i} \bar{A}^{i}\right)
$$

and hence the assertion follows from the continuity of $\widehat{\mathrm{vol}}$.

Remark 6.4.3. (1) By our construction, $\widehat{\operatorname{vol}}(\bar{D})=\widehat{\operatorname{deg}}\left(\bar{D}^{d}\right)$ for $\bar{D} \in \widehat{\operatorname{Nef}}_{C^{0}}(X)_{\mathbb{R}}$. In particular, $\bar{D}$ is big if and only if $\widehat{\operatorname{deg}}\left(\bar{D}^{d}\right)>0$. This is however a non-trivial fact for $\bar{D} \in \widehat{\operatorname{Nef}}_{C^{\infty}}(X)_{\mathbb{R}}$ (cf. [14, Corollary 5.5] and Claim 6.4.2.2).

(2) In [25, Lemma 6.5], [26, Section 1] and [11, Section 5], a symmetric multilinear map

$$
\widehat{\operatorname{Div}}_{C^{0}}^{\mathrm{Nef}}(X) \times \cdots \times \widehat{\operatorname{Div}}_{C^{0}}^{\mathrm{Nef}}(X) \rightarrow \mathbb{R}
$$

is constructed as an extension of

$$
\widehat{\operatorname{Div}}_{C} \infty(X) \times \cdots \times \widehat{\operatorname{Div}}_{C} \infty(X) \rightarrow \mathbb{R}
$$

Of course, it extends by multi-linearity to a symmetric multi-linear map

$$
\widehat{\operatorname{Div}}_{C^{0}}^{\mathrm{Nef}}(X)_{\mathbb{R}} \times \cdots \times \widehat{\operatorname{Div}}_{C^{0}}^{\mathrm{Nef}}(X)_{\mathbb{R}} \rightarrow \mathbb{R} .
$$

Our intersection number in Proposition 6.4.2 coincides with the number given by the above multi-linear map. For details, see [18, Subsections 1.2 and 2.2]. 
Lemma 6.4.4. Let $V$ and $W$ be vector spaces over $\mathbb{R}$ and let $P$ be a cone in $V$, that is, $a x+b y \in P$ whenever $x, y \in P$ and $a, b \in \mathbb{R}_{\geq 0}$ with $a+b>0$. Let $f: P^{s} \rightarrow W$ be a map such that

$$
f\left(x_{1}, \ldots, a x_{i}+b y_{i}, \ldots, x_{s}\right)=a f\left(x_{1}, \ldots, x_{i}, \ldots, x_{s}\right)+b f\left(x_{1}, \ldots, y_{i}, \ldots, x_{s}\right)
$$

for all $i=1, \ldots, s$ and all $x_{1}, \ldots, x_{i}, y_{i}, \ldots, x_{s} \in P$ and $a, b \in \mathbb{R}_{\geq 0}$ with $a+b>0$. If $V=\left\{x-x^{\prime} \mid x, x^{\prime} \in P\right\}$, then there is a unique multi-linear map $\tilde{f}: V^{s} \rightarrow W$ such that $\left.\tilde{f}\right|_{P^{s}}=f$. Moreover, if $f$ is symmetric, then $\tilde{f}$ is also symmetric.

Proof. For $x_{1}, \ldots, x_{s} \in V$, we set $x_{i}=x_{i, 1}-x_{i,-1}\left(x_{i, 1}, x_{i,-1} \in P\right)$ for each $i$, and define

$$
\tilde{f}\left(x_{1}, \ldots, x_{s}\right)=\sum_{\epsilon_{1}, \ldots, \epsilon_{s} \in\{ \pm 1\}} \epsilon_{1} \cdots \epsilon_{s} f\left(x_{1, \epsilon_{1}}, \ldots, x_{s, \epsilon_{s}}\right) .
$$

Claim 6.4.4.1. The above is well-defined, that is, if we choose other $y_{i, 1}, y_{i,-1}$ $\in P$ with $x_{i}=y_{i, 1}-y_{i,-1}$ for each $i$, then

$$
\sum_{\epsilon_{1}, \ldots, \epsilon_{s} \in\{ \pm 1\}} \epsilon_{1} \cdots \epsilon_{s} f\left(x_{1, \epsilon_{1}}, \ldots, x_{s, \epsilon_{s}}\right)=\sum_{\epsilon_{1}, \ldots, \epsilon_{s} \in\{ \pm 1\}} \epsilon_{1} \cdots \epsilon_{s} f\left(y_{1, \epsilon_{1}}, \ldots, y_{s, \epsilon_{s}}\right) .
$$

Proof. For simplicity, we denote

$$
\sum_{\epsilon_{1}, \ldots, \epsilon_{s} \in\{ \pm 1\}} \epsilon_{1} \cdots \epsilon_{s} f\left(x_{1, \epsilon_{1}}, \ldots, x_{s, \epsilon_{s}}\right) \quad \text { and } \sum_{\epsilon_{1}, \ldots, \epsilon_{s} \in\{ \pm 1\}} \epsilon_{1} \cdots \epsilon_{s} f\left(y_{1, \epsilon_{1}}, \ldots, y_{s, \epsilon_{s}}\right)
$$

by $I_{x}$ and $I_{y}$ respectively. We use induction on $s$. If $s=1$, then the assertion is obvious, so that we assume $s>1$. By the induction hypothesis, for all $x \in P$,

$\sum_{\epsilon_{2}, \ldots, \epsilon_{s} \in\{ \pm 1\}} \epsilon_{2} \cdots \epsilon_{s} f\left(x, x_{2, \epsilon_{2}}, \ldots, x_{s, \epsilon_{s}}\right)=\sum_{\epsilon_{2}, \ldots, \epsilon_{s} \in\{ \pm 1\}} \epsilon_{2} \cdots \epsilon_{s} f\left(x, y_{2, \epsilon_{2}}, \ldots, y_{s, \epsilon_{s}}\right)$.

As $x_{1,1}+y_{1,-1}=x_{1,-1}+y_{1,1}$, we have

$$
\begin{aligned}
f\left(x_{1,1}, x_{2, \epsilon_{2}}, \ldots, x_{s, \epsilon_{s}}\right) & +f\left(y_{1,-1}, x_{2, \epsilon_{2}}, \ldots, x_{s, \epsilon_{s}}\right) \\
= & f\left(x_{1,-1}, x_{2, \epsilon_{2}}, \ldots, x_{s, \epsilon_{s}}\right)+f\left(y_{1,1}, x_{2, \epsilon_{2}}, \ldots, x_{s, \epsilon_{s}}\right) .
\end{aligned}
$$

Therefore,

$$
\begin{aligned}
I_{x} & =\sum_{\epsilon_{2}, \ldots, \epsilon_{s} \in\{ \pm 1\}} \epsilon_{2} \cdots \epsilon_{s}\left(f\left(x_{1,1}, x_{2, \epsilon_{2}}, \ldots, x_{s, \epsilon_{s}}\right)-f\left(x_{1,-1}, x_{2, \epsilon_{2}}, \ldots, x_{s, \epsilon_{s}}\right)\right) \\
& =\sum_{\epsilon_{2}, \ldots, \epsilon_{s} \in\{ \pm 1\}} \epsilon_{2} \cdots \epsilon_{s}\left(f\left(y_{1,1}, x_{2, \epsilon_{2}}, \ldots, x_{s, \epsilon_{s}}\right)-f\left(y_{1,-1}, x_{2, \epsilon_{2}}, \ldots, x_{s, \epsilon_{s}}\right)\right) \\
& =\sum_{\epsilon_{2}, \ldots, \epsilon_{s} \in\{ \pm 1\}} \epsilon_{2} \cdots \epsilon_{s}\left(f\left(y_{1,1}, y_{2, \epsilon_{2}}, \ldots, y_{s, \epsilon_{s}}\right)-f\left(y_{1,-1}, y_{2, \epsilon_{2}}, \ldots, y_{s, \epsilon_{s}}\right)\right)=I_{y},
\end{aligned}
$$

as required. 
Clearly, if $f$ is symmetric, then $\tilde{f}$ is also symmetric. The uniqueness and multi-linearity of $\tilde{f}$ are straightforward consequences.

\section{$\S 6.5$. Asymptotic multiplicity}

First we recall the multiplicity of Cartier divisors. Let $(R, \mathfrak{m})$ be a $d$-dimensional noetherian local domain with $d \geq 1$. For a non-zero element $a$ of $R$, we denote the multiplicity of the local ring $(R / a R, \mathfrak{m}(R / a R))$ by $\operatorname{mult}_{\mathfrak{m}}(a)$, that is,

$$
\operatorname{mult}_{\mathfrak{m}}(a):= \begin{cases}\lim _{n \rightarrow \infty} \frac{\text { length }_{R}\left((R / a R) / \mathfrak{m}^{n+1}(R / a R)\right)}{n^{d-1} /(d-1) !} & \text { if } a \notin R^{\times}, \\ 0 & \text { if } a \in R^{\times} .\end{cases}
$$

Note that the above limit always exists and $\operatorname{mult}_{\mathfrak{m}}(a) \in \mathbb{Z}_{\geq 0}$. Moreover, if $R$ is regular, then

$$
\operatorname{mult}_{\mathfrak{m}}(a)=\max \left\{i \in \mathbb{Z}_{\geq 0} \mid a \in \mathfrak{m}^{i}\right\} .
$$

Let $a$ and $b$ be non-zero elements of $R$. By applying [12, Theorem 14.6] to the exact sequence

$$
0 \rightarrow R / a R \stackrel{\times b}{\longrightarrow} R / a b R \rightarrow R / b R \rightarrow 0
$$

we can see that

$$
\operatorname{mult}_{\mathfrak{m}}(a b)=\operatorname{mult}_{\mathfrak{m}}(a)+\operatorname{mult}_{\mathfrak{m}}(b) .
$$

Let $K$ be the quotient field of $R$. For $\alpha \in K^{\times}$, we set $\alpha=a / b(a, b \in R \backslash\{0\})$. Then mult $_{\mathfrak{m}}(a)-$ mult $_{\mathfrak{m}}(b)$ does not depend on the expression $\alpha=a / b$. Indeed, if $\alpha=a / b=a^{\prime} / b^{\prime}$, then, by the previous formula,

$$
\operatorname{mult}_{\mathfrak{m}}(a)+\operatorname{mult}_{\mathfrak{m}}\left(b^{\prime}\right)=\operatorname{mult}_{\mathfrak{m}}\left(a b^{\prime}\right)=\operatorname{mult}_{\mathfrak{m}}\left(a^{\prime} b\right)=\operatorname{mult}_{\mathfrak{m}}\left(a^{\prime}\right)+\operatorname{mult}_{\mathfrak{m}}(b) .
$$

Thus we define

$$
\operatorname{mult}_{\mathfrak{m}}(\alpha):=\operatorname{mult}_{\mathfrak{m}}(a)-\operatorname{mult}_{\mathfrak{m}}(b) .
$$

Note that the map

$$
\text { mult }_{\mathfrak{m}}: K^{\times} \rightarrow \mathbb{Z}
$$

is a homomorphism, that is, $\operatorname{mult}_{\mathfrak{m}}(\alpha \beta)=\operatorname{mult}_{\mathfrak{m}}(\alpha)+\operatorname{mult}_{\mathfrak{m}}(\beta)$ for $\alpha, \beta \in K^{\times}$.

For $x \in X$, we define a homomorphism

$$
\operatorname{mult}_{x}: \operatorname{Div}(X) \rightarrow \mathbb{Z}
$$

by $\operatorname{mult}_{x}(D):=\operatorname{mult}_{\mathfrak{m}_{x}}\left(f_{x}\right)$, where $\mathfrak{m}_{x}$ is the maximal ideal of $\mathscr{O}_{X, x}$ and $f_{x}$ is a local equation of $D$ at $x$. Note that this definition does not depend on the choice of the local equation $f_{x}$. By abuse of notation, the natural extension

$$
\operatorname{mult}_{x} \otimes \operatorname{id}_{\mathbb{R}}: \operatorname{Div}(X)_{\mathbb{R}} \rightarrow \mathbb{R}
$$

is also denoted by mult $_{x}$. 
Let $\bar{D}$ be an arithmetic $\mathbb{R}$-Cartier divisor of $C^{0}$-type. For $x \in X$, we define

$$
\nu_{x}(\bar{D}):= \begin{cases}\inf \left\{\operatorname{mult}_{x}(D+(\phi)) \mid \phi \in \hat{H}^{0}(X, \bar{D}) \backslash\{0\}\right\} & \text { if } \hat{H}^{0}(X, \bar{D}) \neq\{0\} \\ \infty & \text { if } \hat{H}^{0}(X, \bar{D})=\{0\}\end{cases}
$$

We call $\nu_{x}(\bar{D})$ the multiplicity at $x$ of the complete arithmetic linear series of $\bar{D}$. First let us prove the following lemma.

Lemma 6.5.1. Let $\bar{D}$ and $\bar{E}$ be arithmetic $\mathbb{R}$-Cartier divisors of $C^{0}$-type. Then we have the following:

(1) If $\bar{D}$ is effective, then $\nu_{x}(\bar{D}) \leq \operatorname{mult}_{x}(D)$.

(2) $\nu_{x}(\bar{D}+\bar{E}) \leq \nu_{x}(\bar{D})+\nu_{x}(\bar{E})$.

(3) If $\bar{D} \leq \bar{E}$, then $\nu_{x}(\bar{E}) \leq \nu_{x}(\bar{D})+\operatorname{mult}_{x}(E-D)$.

(4) For $\phi \in \operatorname{Rat}(X)^{\times}, \nu_{x}(\bar{D}+\widehat{(\phi)})=\nu_{x}(\bar{D})$.

Proof. (1) is obvious.

(2) If either $\hat{H}^{0}(X, \bar{D})=\{0\}$ or $\hat{H}^{0}(X, \bar{E})=\{0\}$, then the assertion is obvious, so that we may assume that $\hat{H}^{0}(X, \bar{D}) \neq\{0\}$ and $\hat{H}^{0}(X, \bar{E}) \neq\{0\}$. Let $\phi \in$ $\hat{H}^{0}(X, \bar{D}) \backslash\{0\}$ and $\psi \in \hat{H}^{0}(X, \bar{E}) \backslash\{0\}$. Then, as

$$
\widehat{(\phi \psi)}+\bar{E}+\bar{D}=\widehat{(\phi)}+\bar{D}+\widehat{(\psi)}+\bar{E} \geq 0,
$$

we have $\phi \psi \in \hat{H}^{0}(X, \bar{D}+\bar{E}) \backslash\{0\}$. Thus

$$
\nu_{x}(\bar{D}+\bar{E}) \leq \operatorname{mult}_{x}((\phi \psi)+D+E)=\operatorname{mult}_{x}((\phi)+D)+\operatorname{mult}_{x}((\psi)+E),
$$

which implies (2).

(3) If we set $\bar{F}=\bar{E}-\bar{D}$, then, by (1) and (2),

$$
\nu_{x}(\bar{E})=\nu_{x}(\bar{D}+\bar{F}) \leq \nu_{x}(\bar{D})+\nu_{x}(\bar{F}) \leq \nu_{x}(\bar{D})+\operatorname{mult}_{x}(F) .
$$

(4) Let $\alpha: H^{0}(X, D+(\phi)) \rightarrow H^{0}(X, D)$ be the natural isomorphism given by $\alpha(\psi)=(\phi \psi)$. Note that $(\bar{D}+\widehat{(\phi)})+\widehat{(\psi)}=\bar{D}+\widehat{(\alpha(\psi))}$. Thus we have (4).

We set

$$
N(\bar{D})=\left\{n \in \mathbb{Z}_{>0} \mid \hat{H}^{0}(X, n \bar{D}) \neq\{0\}\right\} .
$$

Note that $N(\bar{D})$ is a subsemigroup of $\mathbb{Z}_{>0}$, that is, if $n, m \in N(\bar{D})$, then $n+m \in$ $N(\bar{D})$. We assume that $N(\bar{D}) \neq \emptyset$. For $x \in X$, we define

$$
\mu_{x}(\bar{D}):=\inf \left\{\operatorname{mult}_{x}(D+(1 / n)(\phi)) \mid n \in N(\bar{D}), \phi \in \hat{H}^{0}(X, n \bar{D}) \backslash\{0\}\right\},
$$

which is called the asymptotic multiplicity at $x$ of the complete arithmetic $\mathbb{Q}$-linear series of $\bar{D}$. 
We can see that

$$
\mu_{x}(\bar{D})=\inf \left\{\frac{\nu_{x}(n \bar{D})}{n} \mid n \in N(\bar{D})\right\} .
$$

Indeed, the inequality $\mu_{x}(\bar{D}) \leq \nu_{x}(n \bar{D}) / n$ for $n \in N(\bar{D})$ is obvious, so that $\mu_{x}(\bar{D})$ $\leq \inf \left\{\nu_{x}(n \bar{D}) / n \mid n \in N(\bar{D})\right\}$. Moreover, for $n \in N(\bar{D})$ and $\phi \in \hat{H}^{0}(X, n \bar{D}) \backslash\{0\}$,

$$
\inf \left\{\frac{\nu_{x}(n \bar{D})}{n} \mid n \in N(\bar{D})\right\} \leq \frac{\nu_{x}(n \bar{D})}{n} \leq \operatorname{mult}_{x}(D+(1 / n)(\phi)),
$$

and hence we have the converse inequality.

By the above lemma,

$$
\nu_{x}((n+m) \bar{D}) \leq \nu_{x}(n \bar{D})+\nu_{x}(m \bar{D})
$$

for all $n, m \in N(\bar{D})$. Thus, if $\hat{h}^{0}(\bar{D}) \neq\{0\}$ (i.e., $N(\bar{D})=\mathbb{Z}_{>0}$ ), then

$$
\lim _{n \rightarrow \infty} \frac{\nu_{x}(n \bar{D})}{n}=\inf \left\{\frac{\nu_{x}(n \bar{D})}{n} \mid n>0\right\} .
$$

Proposition 6.5.2. Let $\bar{D}$ and $\bar{E}$ be arithmetic $\mathbb{R}$-Cartier divisors of $C^{0}$-type such that $N(\bar{D}) \neq \emptyset$ and $N(\bar{E}) \neq \emptyset$. Then we have the following:

(1) $\mu_{x}(\bar{D}+\bar{E}) \leq \mu_{x}(\bar{D})+\mu_{x}(\bar{E})$

(2) If $\bar{D} \leq \bar{E}$, then $\mu_{x}(\bar{E}) \leq \mu_{x}(\bar{D})+\operatorname{mult}_{x}(E-D)$.

(3) $\mu_{x}(\bar{D}+\widehat{(\phi)})=\mu_{x}(\bar{D})$ for $\phi \in \operatorname{Rat}(X)^{\times}$.

(4) $\mu_{x}(a \bar{D})=a \mu_{x}(\bar{D})$ for $a \in \mathbb{Q}_{>0}$.

Proof. First let us see (4). We first assume that $a \in \mathbb{Z}_{>0}$. Let $n \in N(\bar{D})$ and $\phi \in \hat{H}^{0}(n \bar{D}) \backslash\{0\}$. Then $\phi^{a} \in \hat{H}^{0}(n(a \bar{D})) \backslash\{0\}$. Thus

$$
\mu_{x}(a \bar{D}) \leq \operatorname{mult}_{x}\left(a D+(1 / n)\left(\phi^{a}\right)\right)=a \operatorname{mult}_{x}(D+(1 / n)(\phi)),
$$

which yields $\mu_{x}(a \bar{D}) \leq a \mu_{x}(\bar{D})$. Conversely, let $n \in N(a \bar{D})$ and $\psi \in \hat{H}^{0}(n(a \bar{D})) \backslash\{0\}$. Then

$$
\mu_{x}(\bar{D}) \leq \operatorname{mult}_{x}(D+(1 / n a)(\psi))=(1 / a) \operatorname{mult}_{x}(a D+(1 / n)(\psi)),
$$

and hence $\mu_{x}(\bar{D}) \leq(1 / a) \mu_{x}(a \bar{D})$. Thus (4) follows in the case where $a \in \mathbb{Z}_{>0}$.

In general, we choose a positive integer $m$ such that $m a \in \mathbb{Z}_{>0}$. Then, by the previous observation,

$$
m \mu_{x}(a \bar{D})=\mu_{x}(m a \bar{D})=\operatorname{ma} \mu_{x}(\bar{D})
$$

as required. 
By (4), to prove (1)-(3) we may assume that $\hat{h}^{0}(\bar{D}) \neq 0$ and $\hat{h}^{0}(\bar{E}) \neq 0$; then the three items follow from (2)-(4) of Lemma 6.5.1 respectively.

Finally, we prove the vanishing of the asymptotic multiplicity for a nef and big arithmetic $\mathbb{R}$-Cartier divisor.

Proposition 6.5.3. If $\bar{D}$ is a nef and big arithmetic $\mathbb{R}$-Cartier divisor of $C^{0}$-type, then $\mu_{x}(\bar{D})=0$ for all $x \in X$.

Proof. Case 1: $\bar{D}$ is an ample arithmetic $\mathbb{R}$-Cartier divisor. Note that if $\bar{D}$ is an ample arithmetic $\mathbb{Q}$-Cartier divisor, then the assertion is obvious. By Lemmas 5.2.3 and 5.2.4, there are $a_{1}, \ldots, a_{l} \in \mathbb{R}$ and effective arithmetic $\mathbb{Q}$-Cartier divisors

$$
\bar{A}_{1}, \ldots, \bar{A}_{l}, \bar{B}_{1}, \ldots, \bar{B}_{l}
$$

of $C^{\infty}$-type such that

$$
\bar{D}=a_{1} \bar{A}_{1}+\cdots+a_{l} \bar{A}_{l}-a_{1} \bar{B}_{1}-\cdots-a_{l} \bar{B}_{l} .
$$

Let us choose sufficiently small positive numbers $\delta_{1}, \ldots, \delta_{l}, \delta_{1}^{\prime}, \ldots, \delta_{l}^{\prime}$ such that $a_{i}-\delta_{i}, a_{i}+\delta_{i}^{\prime} \in \mathbb{Q}$ for all $i$. We set

$$
\bar{D}^{\prime}=\left(a_{1}-\delta_{1}\right) \bar{A}_{1}+\cdots+\left(a_{l}-\delta_{l}\right) \bar{A}_{l}-\left(a_{1}+\delta_{1}^{\prime}\right) \bar{B}_{1}-\cdots-\left(a_{l}+\delta_{l}^{\prime}\right) \bar{B}_{l} .
$$

Then $\bar{D}^{\prime} \leq \bar{D}$ and $\bar{D}^{\prime}$ is an ample arithmetic $\mathbb{Q}$-Cartier divisor by Proposition 6.2.1. By Proposition 6.5.2(2),

$$
0 \leq \mu_{x}(\bar{D}) \leq \mu_{x}\left(\bar{D}^{\prime}\right)+\operatorname{mult}_{x}\left(D-D^{\prime}\right)=\sum\left(\delta_{i} \operatorname{mult}_{x}\left(A_{i}\right)+\delta_{i}^{\prime} \operatorname{mult}_{x}\left(B_{i}\right)\right)
$$

because $\mu_{x}\left(\bar{D}^{\prime}\right)=0$. Therefore,

$$
0 \leq \mu_{x}(\bar{D}) \leq \sum\left(\delta_{i} \operatorname{mult}_{x}\left(A_{i}\right)+\delta_{i}^{\prime} \operatorname{mult}_{x}\left(B_{i}\right)\right),
$$

and hence $\mu_{x}(\bar{D})=0$.

Case 2: $\bar{D}$ is an adequate arithmetic $\mathbb{R}$-Cartier divisor. In this case, there is an ample arithmetic $\mathbb{R}$-Cartier divisor $\bar{A}$ and a non-negative $F_{\infty}$-invariant continuous function $\phi$ on $X(\mathbb{C})$ such that $\bar{D}=\bar{A}+(0, \phi)$. By Proposition 6.5.2(2),

$$
0 \leq \mu_{x}(\bar{D}) \leq \mu_{x}(\bar{A})=0,
$$

as required.

Case 3: General case. Let $\bar{A}$ be an ample arithmetic $\mathbb{Q}$-Cartier divisor. Since $\bar{D}$ is big, by Proposition 6.3.1 there are a positive integer $m$ and $\phi \in \operatorname{Rat}(X)^{\times}$such 
that $\bar{A} \leq m \bar{D}+\widehat{(\phi)}$. We set $\bar{E}=m \bar{D}+\widehat{(\phi)}$. Then $\bar{E}$ is nef. Moreover, for $\delta \in(0,1]$, by Proposition $6.2 .2, \delta \bar{A}+(1-\delta) \bar{E}$ is adequate and $\delta \bar{A}+(1-\delta) \bar{E} \leq \bar{E}$. Hence

$$
\mu_{x}(\bar{E}) \leq \mu_{x}(\delta \bar{A}+(1-\delta) \bar{E})+\delta \operatorname{mult}_{x}(E-A) \leq \delta \operatorname{mult}_{x}(E-A),
$$

which implies that $\mu_{x}(\bar{E})=0$. Therefore, using (3) and (4) of Proposition 6.5.2,

$$
\mu_{x}(\bar{D})=\frac{1}{m} \mu_{x}(m \bar{D})=\frac{1}{m} \mu_{x}(\bar{E})=0 .
$$

\section{§6.6. Generalized Hodge index theorem for an arithmetic $\mathbb{R}$-Cartier divisor}

In this subsection, we prove the following $\mathbb{R}$-Cartier divisor version of $[14$, Corollary 6.4]:

Theorem 6.6.1. Let $\bar{D}$ be an arithmetic $\mathbb{R}$-Cartier divisor of $\left(C^{0} \cap \mathrm{PSH}\right)$-type on $X$. If $D$ is nef on every fiber of $X \rightarrow \operatorname{Spec}(\mathbb{Z})$ (i.e., $\operatorname{deg}\left(\left.D\right|_{C}\right) \geq 0$ for all 1-dimensional closed vertical integral subschemes $C$ on $X)$, then $\widehat{\operatorname{vol}}(\bar{D}) \geq \widehat{\operatorname{deg}}\left(\bar{D}^{d}\right)$.

Proof. Let us begin with the following claim:

Claim 6.6.1.1. Set $\bar{D}=(D, g)$. If $\bar{D}$ is of $C^{\infty}$-type and $D$ is ample (that is, there are $a_{1}, \ldots, a_{l} \in \mathbb{R}_{>0}$ and ample Cartier divisors $A_{1}, \ldots, A_{l}$ such that $D=$ $\left.a_{1} A_{1}+\cdots+a_{l} A_{l}\right)$, and if $d d^{c}([g])+\delta_{D}$ is positive, then the assertion of the theorem holds.

Proof. By Proposition 2.4.2, we can find $F_{\infty}$-invariant locally integrable functions $h_{1}, \ldots, h_{l}$ such that $h_{i}$ is an $A_{i}$-Green function $h_{i}$ of $C^{\infty}$-type for each $i$ and $g=a_{1} h_{1}+\cdots+a_{l} h_{l}$ (a.e.). Let $\delta_{1}, \ldots, \delta_{l}$ be sufficiently small positive real numbers such that $a_{1}+\delta_{1}, \ldots, a_{l}+\delta_{l} \in \mathbb{Q}$. We set

$$
\left(D^{\prime}, g^{\prime}\right)=\left(a_{1}+\delta_{1}\right)\left(A_{1}, h_{1}\right)+\cdots+\left(a_{l}+\delta_{l}\right)\left(A_{l}, h_{l}\right) .
$$

Then $D^{\prime}$ is an ample $\mathbb{Q}$-Cartier divisor, and

$$
d d^{c}\left(\left[g^{\prime}\right]\right)+\delta_{D^{\prime}}=d d^{c}([g])+\delta_{D}+\sum_{i=1}^{l} \delta_{i}\left(d d^{c}\left(\left[h_{i}\right]\right)+\delta_{A_{i}}\right)
$$

is positive because $\delta_{1}, \ldots, \delta_{l}$ are sufficiently small. Therefore, by [14, Corollary 6.4], we have $\widehat{\operatorname{vol}}\left(\bar{D}^{\prime}\right) \geq \widehat{\operatorname{deg}}\left(\bar{D}^{\prime}\right)$, which implies the claim by the continuity of $\widehat{\operatorname{vol}}$ (cf. Theorem 5.2.2).

First we assume that $\bar{D}$ is of $C^{\infty}$-type. Let $\bar{A}=(A, h)$ be an arithmetic Cartier divisor of $C^{\infty}$-type such that $A$ is ample and $d d^{c}([h])+\delta_{A}$ is positive. Then, by 
using the same idea as in the proofs of Propositions 6.2.1 and 6.2.2, we can see that $D+\epsilon A$ is ample for all $\epsilon>0$, Thus, by the above claim, $\widehat{\operatorname{vol}}(\bar{D}+\epsilon(A, h)) \geq$ $\widehat{\operatorname{deg}}\left((\bar{D}+\epsilon(A, h))^{d}\right)$, and hence the assertion follows by letting $\epsilon \rightarrow 0$.

Finally we consider the general case. By Claim 6.4.2.1, there is an ample arithmetic Cartier divisor $\bar{B}$ such that $\bar{A}:=\bar{D}+\bar{B} \in \widehat{\operatorname{Nef}}_{C^{0}}(X)_{\mathbb{R}}$ and $A$ is ample. Let $\epsilon$ be an arbitrary positive number. Then, by Theorem 4.6 , we can find an $F_{\infty^{-}}$ invariant continuous function $u$ on $X(\mathbb{C})$ such that $0 \leq u(x) \leq \epsilon$ for all $x \in X(\mathbb{C})$ and $\bar{A}^{\prime}:=\bar{A}+(0, u) \in \widehat{\operatorname{Div}}_{C^{\infty} \cap \mathrm{PSH}}(X)_{\mathbb{R}}$, which means that $\bar{A}^{\prime} \in \widehat{\operatorname{Nef}}_{C^{\infty}}(X)_{\mathbb{R}}$. Note that

$$
\begin{aligned}
& \widehat{\operatorname{deg}}\left(\bar{D}^{d}\right)=\sum_{i=0}^{d}(-1)^{d-i}\left(\begin{array}{l}
d \\
i
\end{array}\right) \widehat{\operatorname{deg}}\left(\bar{A}^{i} \cdot \bar{B}^{d-i}\right), \\
& \widehat{\operatorname{deg}}\left(\bar{D}^{\prime}{ }^{d}\right)=\sum_{i=0}^{d}(-1)^{d-i}\left(\begin{array}{l}
d \\
i
\end{array}\right) \widehat{\operatorname{deg}}\left(\bar{A}^{\prime i} \cdot \bar{B}^{d-i}\right),
\end{aligned}
$$

where $\bar{D}^{\prime}:=\bar{D}+(0, u)$. By $(6.4 .2 .3), \widehat{\operatorname{deg}}\left(\bar{A}^{i} \cdot \bar{B}^{d-i}\right)$ and $\widehat{\operatorname{deg}}\left(\bar{A}^{\prime i} \cdot \bar{B}^{d-i}\right)$ are given by alternating sums of volumes, so that, by the continuity of $\widehat{\text { vol }}$, there is a constant $C$ not depending on $\epsilon$ such that

$$
\left|\widehat{\operatorname{deg}}\left(\bar{A}^{i} \cdot \bar{B}^{d-i}\right)-\widehat{\operatorname{deg}}\left(\bar{A}^{i} \cdot \bar{B}^{d-i}\right)\right| \leq C \epsilon
$$

for all $i=0, \ldots, d$, and hence

$$
\left|\widehat{\operatorname{deg}}\left(\bar{D}^{\prime d}\right)-\widehat{\operatorname{deg}}\left(\bar{D}^{d}\right)\right| \leq 2^{d} C \epsilon
$$

On the other hand, by the continuity of $\widehat{\text { vol }}$ again, there is a constant $C^{\prime}$ not depending on $\epsilon$ such that

$$
\left|\widehat{\operatorname{vol}}\left(\bar{D}^{\prime}\right)-\widehat{\operatorname{vol}}(\bar{D})\right| \leq C^{\prime} \epsilon
$$

Therefore, by the previous case,

$$
\begin{aligned}
\widehat{\operatorname{vol}}(\bar{D})-\widehat{\operatorname{deg}}\left(\bar{D}^{d}\right) & \left.\geq \widehat{\operatorname{vol}}\left(\bar{D}^{\prime}\right)-C^{\prime} \epsilon\right)-\left(\widehat{\operatorname{deg}}\left(\bar{D}^{\prime} d\right)+2^{d} C \epsilon\right) \\
& =\left(\widehat{\operatorname{vol}}\left(\bar{D}^{\prime}\right)-\widehat{\operatorname{deg}}\left(\bar{D}^{\prime}\right)\right)-\left(C^{\prime}+2^{d} C\right) \epsilon \geq-\left(C^{\prime}+2^{d} C\right) \epsilon .
\end{aligned}
$$

Thus the theorem follows because $\epsilon$ is an arbitrary positive number.

\section{$\S 7$. Limit of nef arithmetic $\mathbb{R}$-Cartier divisors on arithmetic surfaces}

Let $X$ be a regular projective arithmetic surface and let $\mathscr{T}$ be a type for Green functions on $X$ such that PSH is a subjacent type of $\mathscr{T}$. The purpose of this section is to prove the following theorem. 
Theorem 7.1. Let $\left\{\bar{M}_{n}=\left(M_{n}, h_{n}\right)\right\}_{n=0}^{\infty}$ be a sequence of nef arithmetic $\mathbb{R}$ Cartier divisors on $X$ with the following properties:

(a) There is an arithmetic Cartier divisor $\bar{D}=(D, g)$ of $\mathscr{T}$-type such that $g$ is of upper bounded type and $\bar{M}_{n} \leq \bar{D}$ for all $n \geq 1$.

(b) There is a proper closed subset $E$ of $X$ such that $\operatorname{Supp}(D) \subseteq E$ and $\operatorname{Supp}\left(M_{n}\right)$ $\subseteq E$ for all $n \geq 1$.

(c) $\lim _{n \rightarrow \infty} \operatorname{mult}_{C}\left(M_{n}\right)$ exists for all 1-dimensional closed integral subschemes $C$ on $X$.

(d) $\lim \sup _{n \rightarrow \infty}\left(h_{n}\right)_{\text {can }}(x)$ exists in $\mathbb{R}$ for all $x \in X(\mathbb{C}) \backslash E(\mathbb{C})$.

Then there is a nef arithmetic $\mathbb{R}$-Cartier divisor $\bar{M}=(M, h)$ on $X$ such that $\bar{M} \leq \bar{D}$

$$
M=\sum_{C}\left(\lim _{n \rightarrow \infty} \operatorname{mult}_{C}\left(M_{n}\right)\right) C
$$

and $\left.h_{\mathrm{can}}\right|_{X(\mathbb{C}) \backslash E(\mathbb{C})}$ is the upper semicontinuous regularization of the function given by $x \mapsto \limsup _{n \rightarrow \infty}\left(h_{n}\right)_{\text {can }}(x)$ over $X(\mathbb{C}) \backslash E(\mathbb{C})$. Moreover,

$$
\limsup _{n \rightarrow \infty} \widehat{\operatorname{deg}}\left(\left.\bar{M}_{n}\right|_{C}\right) \leq \widehat{\operatorname{deg}}\left(\left.\bar{M}\right|_{C}\right)
$$

for all 1-dimensional closed integral subschemes $C$ on $X$.

Proof. Let $C_{1}, \ldots, C_{l}$ be 1-dimensional irreducible components of $E$. Then there are $a_{1}, \ldots, a_{l}, a_{n 1}, \ldots, a_{n l} \in \mathbb{R}$ such that

$$
D=a_{1} C_{1}+\cdots+a_{l} C_{l} \text { and } M_{n}=a_{n 1} C_{1}+\cdots+a_{n l} C_{l} .
$$

We set $p_{i}=\lim _{n \rightarrow \infty} a_{n i}$ for $i=1, \ldots, l$ and $M=p_{1} C_{1}+\cdots+p_{l} C_{l}$.

Let $U$ be a Zariski open set of $X$ over which we can find local equations $\phi_{1}, \ldots, \phi_{l}$ of $C_{1}, \ldots, C_{l}$ respectively. Let

$$
h_{n}=u_{n}+\sum_{i=1}^{l}\left(-a_{n i}\right) \log \left|\phi_{i}\right|^{2} \text { (a.e.) and } g=v+\sum_{i=1}^{l}\left(-a_{i}\right) \log \left|\phi_{i}\right|^{2} \text { (a.e.) }
$$

be the local expressions of $h_{n}$ and $g$ with respect to $\phi_{1}, \ldots, \phi_{l}$, where $u_{n} \in \mathrm{PSH}_{\mathbb{R}}$ and $v$ is locally bounded above.

Claim 7.1.1. $\left\{u_{n}\right\}_{n=0}^{\infty}$ is locally uniformly bounded above, that is, for each point $x \in U(\mathbb{C})$, there are an open neighborhood $V_{x}$ of $x$ and a constant $M_{x}$ such that $u_{n}(y) \leq M_{x}$ for all $y \in V_{x}$ and $n \geq 0$.

Proof. Since $h_{n} \leq g$ (a.e.), we have

$$
u_{n} \leq v-\sum_{i=1}^{n}\left(a_{i}-a_{n i}\right) \log \left|\phi_{i}\right|^{2} \quad \text { (a.e.) }
$$


over $U(\mathbb{C})$. If $x \notin C_{1}(\mathbb{C}) \cup \cdots \cup C_{l}(\mathbb{C})$, then $\phi_{i}(x) \neq 0$ for all $i$. Thus, as

$$
v-\sum_{i=1}^{n}\left(a_{i}-a_{n i}\right) \log \left|\phi_{i}\right|^{2}
$$

is locally bounded above, the assertion follows from Lemma 2.3.1.

Next we assume that $x \in C_{1}(\mathbb{C}) \cup \cdots \cup C_{l}(\mathbb{C})$. Clearly we may assume that $x \in C_{1}(\mathbb{C})$. Note that $C_{i}(\mathbb{C}) \cap C_{j}(\mathbb{C})=\emptyset$ for $i \neq j$. Thus $\phi_{1}(x)=0$ and $\phi_{i}(x) \neq 0$ for all $i \geq 2$. Therefore, we can find an open neighborhood $V_{x}$ of $x$ and a constant $M_{x}^{\prime}$ such that $\left|\phi_{1}\right|<1$ on $V_{x}$ and

$$
u_{n} \leq M_{x}^{\prime}-\left(a_{1}-a_{n 1}\right) \log \left|\phi_{1}\right|^{2} \quad \text { (a.e.) }
$$

over $V_{x}$ for all $n \geq 1$. Moreover, we can also find a positive constant $M^{\prime \prime}$ such that $a_{1}-a_{n 1} \leq M^{\prime \prime}$ for all $n \geq 1$, so that

$$
u_{n} \leq M_{x}^{\prime}-M^{\prime \prime} \log \left|\phi_{1}\right|^{2} \quad \text { (a.e.) }
$$

over $V_{x}$. Thus the claim follows from Lemma 4.1 .

We set $u(x):=\limsup _{n \rightarrow \infty} u_{n}(x)$ for $x \in U(\mathbb{C})$. Note that $u(x) \in\{-\infty\} \cup \mathbb{R}$. Let $\tilde{u}$ be the upper semicontinuous regularization of $u$. Then, as $u_{n}$ is plurisubharmonic for all $n \geq 1$, by the above claim, $\tilde{u}$ is also plurisubharmonic on $U(\mathbb{C})$ (cf. Subsection 2.1).

Claim 7.1.2. $\tilde{u}(x) \neq-\infty$ for all $x \in U(\mathbb{C})$.

Proof. If $x \notin C_{1}(\mathbb{C}) \cup \cdots \cup C_{l}(\mathbb{C})=E(\mathbb{C})$, then $\phi_{i}(x) \neq 0$ for all $i$. Note that $\lim \sup _{n \rightarrow \infty}\left(h_{n}\right)_{\text {can }}(x)$ exists in $\mathbb{R}$ and that

$$
\left(h_{n}\right)_{\operatorname{can}}(x)=u_{n}(x)+\sum_{i=1}^{l}\left(-a_{n i}\right) \log \left|\phi_{i}(x)\right|^{2} .
$$

Thus $\lim \sup _{n \rightarrow \infty} u_{n}(x)$ exists in $\mathbb{R}$ and

$$
\limsup _{n \rightarrow \infty} u_{n}(x)=\limsup _{n \rightarrow \infty}\left(h_{n}\right)_{\operatorname{can}}(x)+\sum_{i=1}^{l} p_{i} \log \left|\phi_{i}(x)\right|^{2} .
$$

Hence the assertion follows in this case.

Next we assume that $x \in C_{1}(\mathbb{C}) \cup \cdots \cup C_{l}(\mathbb{C})$. We may assume $x \in C_{1}(\mathbb{C})$. As before, $\phi_{1}(x)=0$ and $\phi_{i}(x) \neq 0$ for $i \geq 2$. By using Lemma 5.2.3, let us choose a rational function $\psi$ and effective Cartier divisors $A$ and $B$ such that $C_{1}+(\psi)=A-B$ and $C_{1} \nsubseteq \operatorname{Supp}(A) \cup \operatorname{Supp}(B)$. We set

$$
M_{n}^{\prime}=M_{n}+a_{n 1}(\psi), \quad h_{n}^{\prime}=h_{n}-a_{n 1} \log |\psi|^{2} \quad \text { and } \quad \bar{M}_{n}^{\prime}=\left(M_{n}^{\prime}, h_{n}^{\prime}\right) .
$$


Then $\bar{M}_{n}^{\prime}=\bar{M}_{n}+a_{n 1} \widehat{(\psi)}$ and

$$
\begin{aligned}
0 \leq \widehat{\operatorname{deg}}\left(\left.\bar{M}_{n}\right|_{C_{1}}\right)= & \widehat{\operatorname{deg}}\left(\left.\bar{M}_{n}^{\prime}\right|_{C_{1}}\right) \\
= & a_{n 1}\left(\log \#\left(\mathscr{O}_{C_{1}}(A) / \mathscr{O}_{C_{1}}\right)-\log \#\left(\mathscr{O}_{C_{1}}(B) / \mathscr{O}_{C_{1}}\right)\right) \\
& +\sum_{i=2}^{l} a_{n i} \log \#\left(\mathscr{O}_{C_{1}}\left(C_{i}\right) / \mathscr{O}_{C_{1}}\right)+\frac{1}{2} \sum_{y \in C_{1}(\mathbb{C})}\left(h_{n}^{\prime}\right)_{\operatorname{can}}(y) .
\end{aligned}
$$

Thus we can find a constant $T$ such that

$$
\sum_{y \in C_{1}(\mathbb{C})}\left(h_{n}^{\prime}\right)_{\operatorname{can}}(y) \geq T
$$

for all $n \geq 1$, which yields

$$
\sum_{y \in C_{1}(\mathbb{C})} \limsup _{n \rightarrow \infty}\left(h_{n}^{\prime}\right)_{\text {can }}(y) \geq \limsup _{n \rightarrow \infty}\left(\sum_{y \in C_{1}(\mathbb{C})}\left(h_{n}^{\prime}\right)_{\operatorname{can}}(y)\right) \geq T .
$$

In particular, $\limsup _{n \rightarrow \infty}\left(h_{n}^{\prime}\right)_{\operatorname{can}}(x) \neq-\infty$. On the other hand,

$$
h_{n}^{\prime}=u_{n}-a_{n 1} \log \left|\phi_{1} \psi\right|^{2}-\sum_{i=2}^{l} a_{n i} \log \left|\phi_{i}\right|^{2} \quad \text { (a.e.). }
$$

Note that $\left(\phi_{1} \psi\right)(x) \in \mathbb{C}^{\times}$. Thus

$$
\limsup _{n \rightarrow \infty} u_{n}(x)=\limsup _{n \rightarrow \infty}\left(h_{n}^{\prime}\right)_{\operatorname{can}}(x)+p_{1} \log \left|\left(\phi_{1} \psi\right)(x)\right|^{2}+\sum_{i=2}^{l} p_{i} \log \left|\phi_{i}(x)\right|^{2} .
$$

Therefore we have the assertion of the claim in this case.

Claim 7.1.3. $\tilde{u}+\sum_{i=1}^{l}\left(-p_{i}\right) \log \left|\phi_{i}\right|^{2}$ does not depend on the choice of $\phi_{1}, \ldots, \phi_{l}$.

Proof. Let $\phi_{1}^{\prime}, \ldots, \phi_{l}^{\prime}$ be another set of local equations of $C_{1}, \ldots, C_{l}$. Then there are $e_{1}, \ldots, e_{l} \in \mathscr{O}_{U}^{\times}(U)$ such that $\phi_{i}^{\prime}=e_{i} \phi_{i}$ for all $i$. Let $g_{n}=u_{n}^{\prime}-\sum_{i=1}^{l} a_{n i} \log \left|\phi_{i}^{\prime}\right|^{2}$ (a.e.) be the local expression of $g_{n}$ with respect to $\phi_{1}^{\prime}, \ldots, \phi_{l}^{\prime}$. Then $u_{n}^{\prime}=u_{n}+$ $\sum_{i=1}^{l} a_{n i} \log \left|e_{i}\right|^{2}$ by Lemma 2.3.1. Thus

$$
\tilde{u}^{\prime}=\tilde{u}+\sum_{i=1}^{l} p_{i} \log \left|e_{i}\right|^{2}
$$

which implies that

$$
\tilde{u}+\sum_{i=1}^{l}\left(-p_{i}\right) \log \left|\phi_{i}\right|^{2}=\tilde{u}^{\prime}+\sum_{i=1}^{l}\left(-p_{i}\right) \log \left|\phi_{i}^{\prime}\right|^{2} .
$$


By the above claim, there is an $M$-Green function $h$ of $\mathrm{PSH}_{\mathbb{R}}$-type on $X(\mathbb{C})$ such that

$$
\left.h\right|_{U(\mathbb{C})}=\tilde{u}+\sum_{i=1}^{l}\left(-p_{i}\right) \log \left|\phi_{i}\right|^{2} .
$$

By our construction, $\left.h_{\text {can }}\right|_{X(\mathbb{C}) \backslash E(\mathbb{C})}$ is the upper semicontinuous regularization of the function given by $h^{\sharp}(x)=\lim \sup _{n \rightarrow \infty}\left(h_{n}\right)_{\text {can }}(x)$ over $X(\mathbb{C}) \backslash E(\mathbb{C})$.

Claim 7.1.4. $h$ is $F_{\infty}$-invariant and $h \leq g$ (a.e.).

Proof. As PSH is a subjacent type of $\mathscr{T}$, we have $\left(h_{n}\right)_{\text {can }} \leq g_{\text {can }}$ over $X(\mathbb{C}) \backslash E(\mathbb{C})$, so that $h^{\sharp} \leq g_{\text {can }}$ over $X(\mathbb{C}) \backslash E(\mathbb{C})$. Note that $h^{\sharp}=h$ (a.e.) (cf. Subsection 2.1). Thus the claim follows because $h^{\sharp}$ is $F_{\infty}$-invariant.

Finally let us check that

$$
\widehat{\operatorname{deg}}\left(\left.\bar{M}\right|_{C}\right) \geq \limsup _{n \rightarrow \infty} \widehat{\operatorname{deg}}\left(\left.\bar{M}_{n}\right|_{C}\right) \geq 0
$$

for all 1-dimensional closed integral subschemes $C$ on $X$.

By Lemma 5.2.3 again, we can choose non-zero rational functions $\psi_{1}, \ldots, \psi_{l}$ on $X$ and effective Cartier divisors $A_{1}, \ldots, A_{l}, B_{1}, \ldots, B_{l}$ such that $C_{i}+\left(\psi_{i}\right)=$ $A_{i}-B_{i}$ for all $i$ and $C \nsubseteq \operatorname{Supp}\left(A_{i}\right) \cup \operatorname{Supp}\left(B_{i}\right)$ for all $i$. We set

$$
\begin{aligned}
& M_{n}^{\prime \prime}=M_{n}+\sum_{i=1}^{l} a_{n i}\left(\psi_{i}\right), \quad h_{n}^{\prime \prime}=h_{n}+\sum_{i=1}^{l}\left(-a_{n i}\right) \log \left|\psi_{i}\right|^{2}, \quad \bar{M}_{n}^{\prime \prime}=\left(M_{n}^{\prime \prime}, h_{n}^{\prime \prime}\right), \\
& M^{\prime \prime}=M+\sum_{i=1}^{l} p_{i}\left(\psi_{i}\right), \quad h^{\prime \prime}=h+\sum_{i=1}^{l}\left(-p_{i}\right) \log \left|\psi_{i}\right|^{2}, \quad \bar{M}^{\prime \prime}=\left(M^{\prime \prime}, h^{\prime \prime}\right) .
\end{aligned}
$$

First we assume that $C$ is not flat over $\mathbb{Z}$. Then

$$
\widehat{\operatorname{deg}}\left(\left.\bar{M}_{n}\right|_{C}\right)=\widehat{\operatorname{deg}}\left(\left.\bar{M}_{n}^{\prime \prime}\right|_{C}\right)=\sum_{i=1}^{l} a_{i n}\left(\log \#\left(\mathscr{O}_{C}\left(A_{i}\right) / \mathscr{O}_{C}\right)-\log \#\left(\mathscr{O}_{C}\left(B_{i}\right) / \mathscr{O}_{C}\right)\right)
$$

and

$$
\widehat{\operatorname{deg}}\left(\left.\bar{M}\right|_{C}\right)=\widehat{\operatorname{deg}}\left(\left.\bar{M}^{\prime \prime}\right|_{C}\right)=\sum_{i=1}^{l} p_{i}\left(\log \#\left(\mathscr{O}_{C}\left(A_{i}\right) / \mathscr{O}_{C}\right)-\log \#\left(\mathscr{O}_{C}\left(B_{i}\right) / \mathscr{O}_{C}\right)\right)
$$

Thus

$$
\widehat{\operatorname{deg}}\left(\left.\bar{M}\right|_{C}\right)=\lim _{n \rightarrow \infty} \widehat{\operatorname{deg}}\left(\left.\bar{M}_{n}\right|_{C}\right) \geq 0
$$


Next we assume that $C$ is flat over $\mathbb{Z}$. Then

$$
\begin{aligned}
\widehat{\operatorname{deg}}\left(\left.\bar{M}_{n}\right|_{C}\right) & =\widehat{\operatorname{deg}}\left(\left.\bar{M}_{n}^{\prime \prime}\right|_{C}\right) \\
= & \sum_{i=1}^{l} a_{i n}\left(\log \#\left(\mathscr{O}_{C}\left(A_{i}\right) / \mathscr{O}_{C}\right)-\log \#\left(\mathscr{O}_{C}\left(B_{i}\right) / \mathscr{O}_{C}\right)\right)+\frac{1}{2} \sum_{y \in C(\mathbb{C})}\left(h_{n}^{\prime \prime}\right)_{\operatorname{can}}(y)
\end{aligned}
$$

and

$$
\begin{aligned}
\widehat{\operatorname{deg}}\left(\left.\bar{M}\right|_{C}\right) & =\widehat{\operatorname{deg}}\left(\left.\bar{M}^{\prime \prime}\right|_{C}\right) \\
& =\sum_{i=1}^{l} p_{i}\left(\log \#\left(\mathscr{O}_{C}\left(A_{i}\right) / \mathscr{O}_{C}\right)-\log \#\left(\mathscr{O}_{C}\left(B_{i}\right) / \mathscr{O}_{C}\right)\right)+\frac{1}{2} \sum_{y \in C(\mathbb{C})}\left(h^{\prime \prime}\right)_{\operatorname{can}}(y) .
\end{aligned}
$$

Let us consider a Zariski open set $U$ of $X$ with $C \cap U \neq \emptyset$. Let

$$
h_{n}=u_{n}+\sum\left(-a_{n i}\right) \log \left|\phi_{i}\right|^{2} \text { (a.e.) and } h=\tilde{u}+\sum\left(-p_{i}\right) \log \left|\phi_{i}\right|^{2} \text { (a.e.) }
$$

be the local expressions of $h_{n}$ and $h$ as before. Then

$h_{n}^{\prime \prime}=u_{n}+\sum\left(-a_{n i}\right) \log \left|\phi_{i} \psi_{i}\right|^{2}$ (a.e.) and $\quad h^{\prime \prime}=\tilde{u}+\sum\left(-p_{i}\right) \log \left|\phi_{i} \psi_{i}\right|^{2}$ (a.e.).

Moreover, $\left(\phi_{i} \psi_{i}\right)(y) \in \mathbb{C}^{\times}$for all $y \in C(\mathbb{C})$ and $i$. Thus

$$
\limsup _{n \rightarrow \infty}\left(h_{n}^{\prime \prime}\right)_{\operatorname{can}}(y) \leq\left(h^{\prime \prime}\right)_{\operatorname{can}}(y) \text {. }
$$

Therefore,

$$
\limsup _{n \rightarrow \infty} \sum_{y \in C(\mathbb{C})}\left(h_{n}^{\prime \prime}\right)_{\operatorname{can}}(y) \leq \sum_{y \in C(\mathbb{C})} \limsup _{n \rightarrow \infty}\left(h_{n}^{\prime \prime}\right)_{\operatorname{can}}(y) \leq \sum_{y \in C(\mathbb{C})}\left(h^{\prime \prime}\right)_{\operatorname{can}}(y),
$$

which yields

$$
0 \leq \limsup _{n \rightarrow \infty} \widehat{\operatorname{deg}}\left(\left.\bar{M}_{n}\right|_{C}\right) \leq \widehat{\operatorname{deg}}\left(\left.\bar{M}\right|_{C}\right)
$$

\section{$\S 8$. $\sigma$-decompositions on arithmetic surfaces}

In this section, we consider $\sigma$-decompositions of effective arithmetic $\mathbb{R}$-Cartier divisors of $C^{0}$-type. This is necessary to prove the property (1) of Theorem 9.3.5.

Let $X$ be a regular projective arithmetic surface. We fix an $F_{\infty}$-invariant continuous volume form $\Phi$ on $X(\mathbb{C})$ with $\int_{X(\mathbb{C})} \Phi=1$. Let $\bar{D}=(D, g)$ be an effective arithmetic $\mathbb{R}$-Cartier divisor of $C^{0}$-type on $X$. For a 1-dimensional closed integral subscheme $C$ on $X$, we set

$$
\nu_{C}(\bar{D}):=\min \left\{\operatorname{mult}_{C}(D+(\phi)) \mid \phi \in \hat{H}^{0}(X, \bar{D}) \backslash\{0\}\right\}
$$


as in Subsection 6.5. Moreover, we set

$$
F(\bar{D})=\operatorname{Fx}(\bar{D})=\sum_{C} \nu_{C}(\bar{D}) C \quad \text { and } \quad M(\bar{D})=\operatorname{Mv}(\bar{D})=D-\operatorname{Fx}(\bar{D}) .
$$

Let $V(\bar{D})$ be the complex vector space generated by $\hat{H}^{0}(X, \bar{D})$ in $H^{0}(X, D) \otimes_{\mathbb{Z}} \mathbb{C}$, that is, $V(\bar{D}):=\left\langle\hat{H}^{0}(X, \bar{D})\right\rangle_{\mathbb{C}}$.

Lemma 8.1. $\operatorname{dist}(V(\bar{D}) ; g)$ is $F_{\infty}$-invariant.

Proof. First of all, note that, for $\phi \in \operatorname{Rat}(X), F_{\infty}^{*}(\phi)=\bar{\phi}$ as a function on $X(\mathbb{C})$. Let us check that $\langle\phi, \psi\rangle_{g} \in \mathbb{R}$ for all $\phi, \psi \in\left\langle\hat{H}^{0}(X, \bar{D})\right\rangle_{\mathbb{R}}$. Indeed,

$$
\begin{aligned}
\langle\phi, \psi\rangle_{g} & =\int_{X(\mathbb{C})} \phi \bar{\psi} \exp (-g) \Phi=-\int_{X(\mathbb{C})} F_{\infty}^{*}(\phi \bar{\psi} \exp (-g) \Phi) \\
& =-\int_{X(\mathbb{C})} F_{\infty}^{*}(\phi) F_{\infty}^{*}(\bar{\psi}) F_{\infty}^{*}(\exp (-g)) F_{\infty}^{*}(\Phi) \\
& =\int_{X(\mathbb{C})} \bar{\phi} \psi \exp (-g) \Phi=\langle\psi, \phi\rangle_{g}=\overline{\langle\phi, \psi\rangle_{g}} .
\end{aligned}
$$

Thus $\langle\phi, \psi\rangle_{g}$ yields an inner product on $\left\langle\hat{H}^{0}(X, \bar{D})\right\rangle_{\mathbb{R}}$. Let $\phi_{1}, \ldots, \phi_{N}$ be an orthonormal basis of $\left\langle\hat{H}^{0}(X, \bar{D})\right\rangle_{\mathbb{R}}$ over $\mathbb{R}$. It gives rise to an orthonormal basis of $\left\langle\hat{H}^{0}(X, \bar{D})\right\rangle_{\mathbb{C}}$. Therefore,

$$
\operatorname{dist}(V(\bar{D}) ; g)=\left|\phi_{1}\right|_{g}^{2}+\cdots+\left|\phi_{N}\right|_{g}^{2}
$$

Note that $F_{\infty}^{*}\left(\left|\phi_{i}\right|_{g}\right)=\left|\bar{\phi}_{i}\right|_{g}=\left|\phi_{i}\right|_{g}$, and hence the lemma follows.

Here we define $g_{F(\bar{D})}, g_{M(\bar{D})}, \bar{M}(\bar{D})$ and $\bar{F}(\bar{D})$ as follows:

$$
\begin{array}{llrl}
g_{F(\bar{D})}=-\log \operatorname{dist}(V(\bar{D}) ; g), & g_{M(\bar{D})}=g-g_{F(\bar{D})}=g+\log \operatorname{dist}(V(\bar{D}) ; g), \\
\bar{M}(\bar{D})=\left(M(\bar{D}), g_{M(\bar{D})}\right), & \bar{F}(\bar{D})=\left(F(\bar{D}), g_{F(\bar{D})}\right) .
\end{array}
$$

Let us check the following proposition:

Proposition 8.2. (1) $\hat{H}^{0}(X, \bar{D}) \subseteq \hat{H}^{0}(X, \bar{M}(\bar{D}))$.

(2) $g_{M(\bar{D})}$ is an $M(\bar{D})$-Green function of $\left(C^{\infty} \cap \mathrm{PSH}\right)$-type on $X(\mathbb{C})$.

(3) $g_{F(\bar{D})}$ is an $F(\bar{D})$-Green function of $\left(C^{0}-C^{\infty} \cap \mathrm{PSH}\right)$-type over $X(\mathbb{C})$.

(4) $\bar{M}(\bar{D})$ is nef.

Proof. (1) If $\phi \in \hat{H}^{0}(X, \bar{D}) \backslash\{0\}$, then $(\phi)+D \geq F(\bar{D})$, and hence $(\phi)+M(\bar{D}) \geq 0$. Note that $|\phi|_{g}^{2}=\operatorname{dist}(V(\bar{D}) ; g)|\phi|_{g_{M(\bar{D})}}^{2}$ for $\phi \in \hat{H}^{0}(X, M(\bar{D}))$. Thus, as $\|\phi\|_{g} \leq 1$, by Proposition 3.2.1 we have $|\phi|_{g_{M(\bar{D})}}^{2}=|\phi|_{g}^{2} / \operatorname{dist}(V(\bar{D}) ; g) \leq\|\phi\|_{g}^{2} \leq 1$. Therefore, $\phi \in \hat{H}^{0}(X, \bar{M}(\bar{D}))$. 
(2), (3) Let us fix $x \in X(\mathbb{C})$. We set

$$
\nu_{x}:=\min \left\{\operatorname{mult}_{x}(D+(\phi)) \mid \phi \in V(\bar{D}) \backslash\{0\}\right\} .
$$

Note that $\operatorname{mult}_{x}(D+(\phi))=\operatorname{mult}_{x}(D)+\operatorname{ord}_{x}(\phi)$. First let us prove the following claim:

Claim 8.2.1. (a) If $\phi_{1}, \ldots, \phi_{n} \in V(\bar{D}) \backslash\{0\}$ and $V(\bar{D})$ is generated by $\phi_{1}, \ldots, \phi_{n}$, then $\nu_{x}=\min \left\{\operatorname{mult}_{x}\left(D+\left(\phi_{1}\right)\right), \ldots, \operatorname{mult}_{x}\left(D+\left(\phi_{n}\right)\right)\right\}$.

(b) $\nu_{x}=\operatorname{mult}_{x}(F(\bar{D}))$.

Proof. (a) is obvious. Let us consider the natural homomorphism

$$
\left\langle\hat{H}^{0}(X, \bar{D})\right\rangle_{\mathbb{Z}} \otimes_{\mathbb{Z}} \mathscr{O}_{X} \rightarrow \mathscr{O}_{X}(\lfloor D\rfloor),
$$

which is surjective on $X \backslash \operatorname{Supp}(D)$ because $0 \leq\lfloor D\rfloor \leq D$. In particular,

$$
V(\bar{D}) \otimes_{\mathbb{C}} \mathscr{O}_{X(\mathbb{C})} \rightarrow \mathscr{O}_{X(\mathbb{C})}(\lfloor D\rfloor)
$$

is surjective on $X(\mathbb{C}) \backslash \operatorname{Supp}(D)(\mathbb{C})$, so that if $x \in X(\mathbb{C}) \backslash \operatorname{Supp}(D)(\mathbb{C})$, then $\nu_{x}=0$. On the other hand, if $x \in X(\mathbb{C}) \backslash \operatorname{Supp}(D)(\mathbb{C})$, then $\operatorname{mult}_{x}(F(\bar{D}))=0$ because $0 \leq F(\bar{D}) \leq D$. Therefore, we may assume that $x \in \operatorname{Supp}(D)(\mathbb{C})$, so that there is a 1-dimensional closed integral subscheme $C$ of $X$ with $x \in C(\mathbb{C})$. Let $\psi_{1}, \ldots, \psi_{n}$ be all elements of $\hat{H}^{0}(X, \bar{D}) \backslash\{0\}$. Let $\eta$ be the generic point of $C$. Then

$$
\operatorname{mult}_{C}(F(\bar{D}))=\min \left\{\operatorname{mult}_{C}(D)+\operatorname{ord}_{\eta}\left(\psi_{1}\right), \ldots, \operatorname{mult}_{C}(D)+\operatorname{ord}_{\eta}\left(\psi_{n}\right)\right\}
$$

Thus, by (a),

$$
\begin{aligned}
\operatorname{mult}_{x}(F(\bar{D})) & =\operatorname{mult}_{C}(F(\bar{D})) \\
& =\min \left\{\operatorname{mult}_{C}(D)+\operatorname{ord}_{\eta}\left(\psi_{1}\right), \ldots, \operatorname{mult}_{C}(D)+\operatorname{ord}_{\eta}\left(\psi_{n}\right)\right\} \\
& =\min \left\{\operatorname{mult}_{x}\left(D+\left(\psi_{1}\right)\right), \ldots, \operatorname{mult}_{x}\left(D+\left(\psi_{n}\right)\right)\right\}=\nu_{x} .
\end{aligned}
$$

Let $\phi_{1}, \ldots, \phi_{N}$ be an orthonormal basis of $V(\bar{D})$ with respect to $\langle,\rangle_{g}$. Let $g=u_{x}+(-a) \log |z|^{2}$ (a.e.) be a local expression of $g$ around $x$, where $z$ is a local chart around $x$ with $z(x)=0$. For every $i$, we set $\phi_{i}=z^{a_{i}} v_{i}$ around $x$ with $v_{i} \in \mathscr{O}_{X(\mathbb{C}), x}^{\times}$. Then $\left|\phi_{i}\right|_{g}^{2}=|z|^{2\left(a_{i}+a\right)} \exp \left(-u_{x}\right)\left|v_{i}\right|^{2}$. By the above claim,

$$
\nu_{x}=\min \left\{a_{1}+a, \ldots, a_{N}+a\right\}=\operatorname{mult}_{x}(F(\bar{D})) .
$$

Thus

$$
\operatorname{dist}(V(\bar{D}) ; g)=|z|^{2 \nu_{x}} \exp \left(-u_{x}\right) \sum_{i=1}^{N}|z|^{2\left(a_{i}+a-\nu_{x}\right)}\left|v_{i}\right|^{2}
$$


Therefore,

$$
\begin{aligned}
& g_{F(\bar{D})}=u_{x}-\log \left(\sum_{i=1}^{N}|z|^{2\left(a_{i}+a-\nu_{x}\right)}\left|v_{i}\right|^{2}\right)-\nu_{x} \log |z|^{2} \\
& g_{M(\bar{D})}=\log \left(\sum_{i=1}^{N}|z|^{2\left(a_{i}+a-\nu_{x}\right)}\left|v_{i}\right|^{2}\right)-\left(a-\nu_{x}\right) \log |z|^{2} .
\end{aligned}
$$

Note that $\log \left(\sum_{i=1}^{N}|z|^{2\left(a_{i}+a-\nu_{x}\right)}\left|v_{i}\right|^{2}\right)$ is a subharmonic $C^{\infty}$-function. Thus we get (2) and (3).

(4) For $\phi \in \hat{H}^{0}(X, \bar{D}) \backslash\{0\}$ and a 1-dimensional closed integral subscheme $C$ on $X$, as

$$
\operatorname{mult}_{C}(M(\bar{D})+(\phi))=\operatorname{mult}_{C}(D+(\phi))-\nu_{C}(\bar{D}),
$$

there is a $\psi \in \hat{H}^{0}(X, \bar{D}) \backslash\{0\}$ such that $\operatorname{mult}_{C}(M(\bar{D})+(\psi))=0$. This means that

$$
C \not \subset \operatorname{Supp}(M(\bar{D})+(\psi)) \text {. }
$$

Then, by Proposition 3.2.1, $0<|\psi|_{g_{M(\bar{D})}}(x) \leq 1$ for all $x \in C(\mathbb{C})$ as before. Hence

$$
\widehat{\operatorname{deg}}\left(\left.\bar{M}(\bar{D})\right|_{C}\right)=\log \# \mathscr{O}_{C}((\psi)+M(\bar{D})) / \mathscr{O}_{C}-\sum_{x \in C(\mathbb{C})} \log |\psi|_{g_{M(\bar{D})}}(x) \geq 0
$$

For $n \geq 1$, we set

$$
\begin{aligned}
M_{n}(\bar{D}) & :=\frac{1}{n} M(n \bar{D}), & g_{M_{n}(\bar{D})} & :=\frac{1}{n} g_{M(n \bar{D})}, \\
F_{n}(\bar{D}) & :=\frac{1}{n} F(n \bar{D}), & g_{F_{n}(\bar{D})} & :=\frac{1}{n} g_{F(n \bar{D})} .
\end{aligned}
$$

In addition,

$$
\bar{M}_{n}(\bar{D}):=\left(M_{n}(\bar{D}), g_{M_{n}(\bar{D})}\right) \quad \text { and } \quad \bar{F}_{n}(\bar{D}):=\left(F_{n}(\bar{D}), g_{F_{n}(\bar{D})}\right)
$$

Then we have the following proposition, which guarantees a decomposition

$$
\bar{D}=\bar{M}_{\infty}(\bar{D})+\bar{F}_{\infty}(\bar{D})
$$

as described in the proposition. This is called the $\sigma$-decomposition of $\bar{D}$. Moreover, $\bar{M}_{\infty}(\bar{D})$ (resp. $\bar{F}_{\infty}(\bar{D})$ ) is called the asymptotic movable part (resp. the asymptotic fixed part) of $\bar{D}$. The $\sigma$-decomposition is an arithmetic analog of the $\sigma$-decomposition introduced by Nakayama [21].

Proposition 8.3. There exists a nef arithmetic $\mathbb{R}$-Cartier divisor $\bar{M}_{\infty}(\bar{D})=$ $\left(M_{\infty}(\bar{D}), g_{M_{\infty}(\bar{D})}\right)$ on $X$ with the following properties:

(1) $\operatorname{mult}_{C}\left(M_{\infty}(\bar{D})\right)=\lim _{n \rightarrow \infty} \operatorname{mult}_{C}\left(M_{n}(\bar{D})\right)$ for all 1-dimensional closed integral subschemes $C$ on $X$. 
(2) $\left(g_{M_{\infty}(\bar{D})}\right)_{\text {can }}$ is the upper semicontinuous regularization of the function given by

$$
x \mapsto \limsup _{n \rightarrow \infty}\left(g_{M_{n}(\bar{D})}\right)_{\operatorname{can}}(x)
$$

over $X(\mathbb{C}) \backslash \operatorname{Supp}(D)(\mathbb{C})$. In particular,

$$
\left(g_{M_{\infty}(\bar{D})}\right)_{\operatorname{can}}(x)=\limsup _{n \rightarrow \infty}\left(g_{M_{n}(\bar{D})}\right)_{\operatorname{can}}(x) \quad \text { (a.e.). }
$$

Moreover, if $\bar{D}$ is of $C^{\infty}$-type, then $\lim _{n \rightarrow \infty}\left(g_{M_{n}(\bar{D})}\right)_{\operatorname{can}}(x)$ exists.

(3) $\widehat{\operatorname{deg}}\left(\left.\bar{M}_{\infty}(\bar{D})\right|_{C}\right) \geq \lim \sup _{n \rightarrow \infty} \widehat{\operatorname{deg}}\left(\left.\bar{M}_{n}(\bar{D})\right|_{C}\right)$ for all 1-dimensional closed integral subschemes $C$ on $X$.

(4) Define

$$
\begin{aligned}
F_{\infty}(\bar{D}) & :=D-M_{\infty}(\bar{D}), \\
g_{F_{\infty}(\bar{D})} & :=g-g_{M_{\infty}(\bar{D})}, \\
\bar{F}_{\infty}(\bar{D}) & :=\left(F_{\infty}(\bar{D}), g_{F_{\infty}(\bar{D})}\right)\left(=\bar{D}-\bar{M}_{\infty}(\bar{D})\right) .
\end{aligned}
$$

Then $\mu_{C}(\bar{D})=\operatorname{mult}_{C}\left(F_{\infty}(\bar{D})\right)$ for all 1-dimensional closed integral subschemes $C$ on $X$, and $\bar{F}_{\infty}(\bar{D})$ is an effective arithmetic $\mathbb{R}$-Cartier divisor of $\left(C^{0}-\mathrm{PSH}_{\mathbb{R}}\right)$-type. In addition, if $\bar{D}$ is of $C^{\infty}$-type, then there is a constant e such that

$$
\left.n g_{F_{\infty}(\bar{D})} \leq g_{F(n \bar{D})}+3 \log (n+1)+e \quad \text { (a.e. }\right)
$$

for all $n \geq 1$.

(5) If $\bar{D}$ is of $C^{\infty}$-type, then there is a constant $e^{\prime}$ such that

$$
\hat{h}^{0}\left(X, n \bar{M}_{\infty}(\bar{D})\right) \leq \hat{h}^{0}(X, n \bar{D}) \leq \hat{h}^{0}\left(X, n \bar{M}_{\infty}(\bar{D})\right)+e^{\prime} n \log (n+1)
$$

for all $n \geq 1$.

Proof. It is easy to see that

$$
\operatorname{mult}_{C}(F((n+m) \bar{D})) \leq \operatorname{mult}_{C}(F(n \bar{D}))+\operatorname{mult}_{C}(F(m \bar{D}))
$$

for all $n, m \geq 1$ and all 1-dimensional closed integral subschemes $C$. Thus the limit $\lim _{n \rightarrow \infty} \operatorname{mult}_{C}\left(F_{n}(\bar{D})\right)$ exists and

$$
\lim _{n \rightarrow \infty} \operatorname{mult}_{C}\left(F_{n}(\bar{D})\right)=\inf _{n \geq 1} \operatorname{mult}_{C}\left(F_{n}(\bar{D})\right) .
$$

Therefore $\lim _{n \rightarrow \infty} \operatorname{mult}_{C}\left(M_{n}(\bar{D})\right)$ exists because $M_{n}(\bar{D})=D-F_{n}(\bar{D})$. Note that $\mu_{C}(\bar{D})=\lim _{n \rightarrow \infty} \operatorname{mult}_{C}\left(F_{n}(\bar{D})\right)$ as $\operatorname{mult}_{C}\left(F_{n}(\bar{D})\right)=\nu_{C}(\bar{D}) / n$ (cf. Subsection 6.5). 
Claim 8.3.1. Let $h$ be a D-Green function of $C^{\infty}$-type. Then there is a positive constant $A$ such that, for $x \in X(\mathbb{C}) \backslash \operatorname{Supp}(D)(\mathbb{C})$, the limit

$$
\lim _{n \rightarrow \infty} \frac{\log (\operatorname{dist}(V(n \bar{D}) ; n h)(x))}{n}
$$

exists in $\mathbb{R}_{\leq 0}$ and

$$
\begin{aligned}
\lim _{n \rightarrow \infty} \frac{\log (\operatorname{dist}(V(n \bar{D}) ; n h)(x))-\log \left(A(n+1)^{3}\right)}{n} \\
\quad=\sup _{n \geq 1} \frac{\log (\operatorname{dist}(V(n \bar{D}) ; n h)(x))-\log \left(A(n+1)^{3}\right)}{n} .
\end{aligned}
$$

Proof. First, note that $\bigoplus_{n=0}^{\infty} V(n \bar{D})$ is a graded subring of $\bigoplus_{n=0}^{\infty} H^{0}(X, n D)$. By Theorem 3.2.3, there is a positive constant $A$ such that

$$
\operatorname{dist}(V(n \bar{D}) ; n h) \leq A(n+1)^{3}
$$

and

$$
\frac{\operatorname{dist}(V(n \bar{D}) ; n h)}{A(n+1)^{3}} \cdot \frac{\operatorname{dist}(V(m \bar{D}) ; m h)}{A(m+1)^{3}} \leq \frac{\operatorname{dist}(V((n+m) \bar{D}) ;(n+m) h)}{A(n+m+1)^{3}}
$$

for all $n, m \geq 1$. Moreover, $\operatorname{dist}(V(n \bar{D}) ; n h)(x) \neq 0$ for $x \in X(\mathbb{C}) \backslash \operatorname{Supp}(D)(\mathbb{C})$. Thus the claim follows.

By using the Stone-Weierstrass theorem, for any positive number $\epsilon$, we can find continuous functions $u$ and $v$ with the following properties:

$$
\begin{aligned}
& u \geq 0, \quad\|u\|_{\text {sup }} \leq \epsilon, \quad h:=g+u \text { is of } C^{\infty} \text {-type } \\
& v \geq 0, \quad\|v\|_{\text {sup }} \leq \epsilon, \quad h^{\prime}:=g-v \text { is of } C^{\infty} \text {-type. }
\end{aligned}
$$

By Lemma 3.2.2,

$$
\exp (-n \epsilon) \operatorname{dist}\left(V(n \bar{D}) ; n h^{\prime}\right) \leq \operatorname{dist}(V(n \bar{D}) ; n g) \leq \exp (n \epsilon) \operatorname{dist}(V(n \bar{D}) ; n h) .
$$

Thus, by the above claim, for $x \in X(\mathbb{C}) \backslash \operatorname{Supp}(D)(\mathbb{C})$,

$$
\limsup _{n \rightarrow \infty} \frac{\log (\operatorname{dist}(V(n \bar{D}) ; n g)(x))}{n}
$$

exists in $\{a \in \mathbb{R} \mid a \leq \epsilon\}$. Since $\epsilon$ is an arbitrary positive number, we actually have

$$
\limsup _{n \rightarrow \infty} \frac{\log (\operatorname{dist}(V(n \bar{D}) ; n g)(x))}{n} \leq 0 \text {. }
$$


This shows that $\lim \sup _{n \rightarrow \infty}\left(g_{M_{n}(\bar{D})}\right)_{\text {can }}(x)$ exists in $\mathbb{R}$ for $x \in X(\mathbb{C}) \backslash \operatorname{Supp}(D)(\mathbb{C})$. Therefore, by Theorem 7.1 , there is a nef arithmetic $\mathbb{R}$-Cartier divisor

$$
\bar{M}_{\infty}(\bar{D})=\left(M_{\infty}(\bar{D}), g_{M_{\infty}(\bar{D})}\right)
$$

satisfying (1)-(3). Further the last assertion of (2) is a consequence of the above claim.

Let us show (4). Obviously $\mu_{C}(\bar{D})=\operatorname{mult}_{C}\left(F_{\infty}(\bar{D})\right)$ because

$$
\mu_{C}(\bar{D})=\lim _{n \rightarrow \infty} \operatorname{mult}_{C}\left(F_{n}(\bar{D})\right) .
$$

Note that

$$
\left(g_{F_{\infty}(\bar{D})}\right)_{\operatorname{can}}(x)=-\limsup _{n \rightarrow \infty} \frac{\log (\operatorname{dist}(V(n \bar{D}) ; n g)(x))}{n} \quad \text { (a.e.) }
$$

on $X(\mathbb{C}) \backslash \operatorname{Supp}(D)(\mathbb{C})$. Thus $(8.3 .2)$ yields $\left(g_{F_{\infty}(\bar{D})}\right)_{\operatorname{can}}(x) \geq 0$ (a.e.). Hence $\bar{F}_{\infty}(\bar{D})$ is effective. Moreover, it is obvious that $g_{F_{\infty}(\bar{D})}$ is of $\left(C^{0}-\mathrm{PSH}_{\mathbb{R}}\right)$-type because $g$ is of $C^{0}$-type and $g_{M_{\infty}(\bar{D})}$ is of $\mathrm{PSH}_{\mathbb{R}^{-} \text {-type. }}$

Now assume that $\bar{D}$ is of $C^{\infty}$-type. By the above claim, there is a positive constant $A^{\prime}$ such that

$$
\begin{aligned}
-\lim _{n \rightarrow \infty} \frac{\log (\operatorname{dist}(V(n \bar{D}) ; n g)(x))}{n} \\
\quad=\lim _{n \rightarrow \infty} \frac{-\log (\operatorname{dist}(V(n \bar{D}) ; n g)(x))+\log \left(A^{\prime}(n+1)^{3}\right)}{n} \\
\quad=\inf _{n \geq 1} \frac{-\log (\operatorname{dist}(V(n \bar{D}) ; n g)(x))+\log \left(A^{\prime}(n+1)^{3}\right)}{n}
\end{aligned}
$$

on $X(\mathbb{C}) \backslash \operatorname{Supp}(D)(\mathbb{C})$. Thus, for $n \geq 1$,

$$
g_{F_{\infty}(\bar{D})} \leq \frac{-\log (\operatorname{dist}(V(n \bar{D}) ; n g)(x))+\log \left(A^{\prime}(n+1)^{3}\right)}{n} \quad \text { (a.e.) }
$$

which implies the last assertion of (4).

Finally let us check (5). By (4), we have $\bar{M}_{\infty}(\bar{D}) \leq \bar{D}$, so that

$$
\hat{h}^{0}\left(X, n \bar{M}_{\infty}(\bar{D})\right) \leq \hat{h}^{0}(X, n \bar{D})
$$

for $n \geq 1$. Moreover, by (4) again,

$$
n \bar{M}_{\infty}(\bar{D})+\left(0.3 \log (n+1)+\log A^{\prime}\right) \geq \bar{M}(n \bar{D})
$$

for all $n \geq 1$. Thus, by Proposition 8.2(1),

$$
\hat{h}^{0}(X, n \bar{D}) \leq \hat{h}^{0}(X, \bar{M}(n \bar{D})) \leq \hat{h}^{0}\left(X, n \bar{M}_{\infty}(\bar{D})+\left(0.3 \log (n+1)+\log A^{\prime}\right)\right) .
$$


Note that there is a positive constant $e^{\prime}$ such that

$$
\hat{h}^{0}\left(X, n \bar{M}_{\infty}(\bar{D})+\left(0.3 \log (n+1)+\log A^{\prime}\right)\right) \leq \hat{h}^{0}\left(X, n \bar{M}_{\infty}(\bar{D})\right)+e^{\prime} n \log (n+1)
$$

for all $n \geq 1$ (cf. [14, Proposition 2.1(3)] and [16, Lemma 1.2.2]). Thus (5) follows.

\section{§9. Zariski decompositions and their properties on arithmetic surfaces}

Throughout this section, $X$ is a regular projective arithmetic surface and $\mathscr{T}$ is a type for Green functions on $X$. We always assume that PSH is a subjacent type of $\mathscr{T}$.

\section{§9.1. Preliminaries}

In this subsection, we prepare several lemmas for the proof of Theorem 9.2.1.

Lemma 9.1.1. Assume that $\mathscr{T}$ is either $C^{0}$ or $\mathrm{PSH}_{\mathbb{R}}$. Let $M$ be a 1-equidimensional complex manifold and let $D_{1}, \ldots, D_{n}$ be $\mathbb{R}$-Cartier divisors on $M$. Let $g_{1}, \ldots, g_{n}$ be locally integrable functions on $M$ such that $g_{i}$ is a $D_{i}$-Green function of $\mathscr{T}$-type for each $i$. Set

$$
g(x)=\max \left\{g_{1}(x), \ldots, g_{n}(x)\right\} \quad(x \in M)
$$

and

$$
D=\sum_{x \in M} \max \left\{\operatorname{mult}_{x}\left(D_{1}\right), \ldots, \operatorname{mult}_{x}\left(D_{n}\right)\right\} x .
$$

Then $g$ is a D-Green function of $\mathscr{T}$-type.

Proof. For $x \in M$, let $z$ be a local chart of an open neighborhood $U_{x}$ of $x$ with $z(x)=0$, and let

$$
g_{1}=u_{1}-a_{1} \log |z|^{2} \text { (a.e.), } \ldots, g_{n}=u_{n}-a_{n} \log |z|^{2} \text { (a.e.) }
$$

be local expressions of $g_{1}, \ldots, g_{n}$ respectively over $U_{x}$, where $a_{i}=\operatorname{mult}_{x}\left(D_{i}\right)$ and $u_{i} \in \mathscr{T}\left(U_{x}\right)$ for $i=1, \ldots, n$. Clearly we may assume that $a_{1}=\max \left\{a_{1}, \ldots, a_{n}\right\}$. First of all, we have

$$
g=\max \left\{u_{i}+\left(a_{1}-a_{i}\right) \log |z|^{2} \mid i=1, \ldots, n\right\}-a_{1} \log |z|^{2} \quad \text { (a.e.) }
$$

over $U_{x}$. In addition, the value of

$$
u:=\max \left\{u_{i}+\left(a_{1}-a_{i}\right) \log |z|^{2} \mid i=1, \ldots, n\right\}
$$

at $y \in U_{x}$ is finite. 
First we consider the case where $\mathscr{T}=\mathrm{PSH}_{\mathbb{R}}$. Then $u_{1}, \ldots, u_{n}$ are subharmonic over $U_{x}$, so that $u_{i}+\left(a_{1}-a_{i}\right) \log |z|^{2}$ is also subharmonic over $U_{x}$ for every $i$. Therefore, $u$ is subharmonic over $U_{x}$.

Next let us consider the case where $\mathscr{T}=C^{0}$. We set $I=\left\{i \mid a_{i}=a_{1}\right\}$. Then, shrinking $U_{x}$ if necessary, we may assume that $u_{1}>u_{j}+\left(a_{1}-a_{j}\right) \log |z|^{2}$ on $U_{x}$ for all $j \notin I$. Thus $u=\max \left\{u_{i} \mid i \in I\right\}$, and hence $u$ is continuous.

Lemma 9.1.2. Assume that $\mathscr{T}$ is either $C^{0}$ or $\mathrm{PSH}_{\mathbb{R}}$. Let

$$
\bar{D}_{1}=\left(D_{1}, g_{1}\right), \ldots, \bar{D}_{n}=\left(D_{n}, g_{n}\right)
$$

be arithmetic $\mathbb{R}$-Cartier divisors of $\mathscr{T}$-type on $X$. Set

$$
\begin{aligned}
\max \left\{D_{1}, \ldots, D_{n}\right\}: & =\sum_{C} \max \left\{\operatorname{mult}_{C}\left(D_{1}\right), \ldots, \operatorname{mult}_{C}\left(D_{n}\right)\right\} C, \\
\max \left\{\bar{D}_{1}, \ldots, \bar{D}_{n}\right\}: & :=\left(\max \left\{D_{1}, \ldots, D_{n}\right\}, \max \left\{g_{1}, \ldots, g_{n}\right\}\right) .
\end{aligned}
$$

Then we have the following:

(1) $\max \left\{\bar{D}_{1}, \ldots, \bar{D}_{n}\right\}$ is an arithmetic $\mathbb{R}$-Cartier divisor of $\mathscr{T}$-type for $D$.

(2) If $\mathscr{T}=\operatorname{PSH}_{\mathbb{R}}$ and $\bar{D}_{1}, \ldots, \bar{D}_{n}$ are nef, then $\max \left\{\bar{D}_{1}, \ldots, \bar{D}_{n}\right\}$ is nef.

Proof. (1) It is obvious that $\max \left\{g_{1}, \ldots, g_{n}\right\}$ is $F_{\infty}$-invariant, so that (1) follows from Lemma 9.1.1.

(2) For simplicity, we set $D=\max \left\{D_{1}, \ldots, D_{n}\right\}, g=\max \left\{g_{1}, \ldots, g_{n}\right\}$ and $\bar{D}=\max \left\{\bar{D}_{1}, \ldots, \bar{D}_{n}\right\}$. Let $C$ be a 1-dimensional closed integral subscheme of $X$. Let $\gamma$ be the generic point of $C$. Since the codimension of

$$
\operatorname{Supp}\left(D-D_{1}\right) \cap \cdots \cap \operatorname{Supp}\left(D-D_{n}\right)
$$

is greater than or equal to 2 , there is $i$ such that $\gamma \notin \operatorname{Supp}\left(D-D_{i}\right)$. By Proposition 2.3.4, $g-g_{i}$ is a $\left(D-D_{i}\right)$-Green function of $\left(\mathrm{PSH}_{\mathbb{R}}-\mathrm{PSH}_{\mathbb{R}}\right)$-type and $g-g_{i} \geq 0$ (a.e.). Moreover, as $x \notin \operatorname{Supp}\left(D-D_{i}\right)$ for $x \in C(\mathbb{C})$, by Proposition 2.3.4 we have

$$
\left(g-g_{i}\right)_{\operatorname{can}}(x) \geq 0 .
$$

Therefore, $\widehat{\operatorname{deg}}\left(\bar{D}-\left.\bar{D}_{i}\right|_{C}\right) \geq 0$, and hence

$$
\widehat{\operatorname{deg}}\left(\left.\bar{D}\right|_{C}\right) \geq \widehat{\operatorname{deg}}\left(\left.\bar{D}_{i}\right|_{C}\right) \geq 0 \text {. }
$$

Lemma 9.1.3. Let $(D, g)$ be an effective arithmetic $\mathbb{R}$-Cartier divisor of $C^{0}$-type on $X$ and let $E$ be an $\mathbb{R}$-Cartier divisor on $X$ with $0 \leq E \leq D$. Then there is an $F_{\infty}$-invariant $E$-Green function $h$ of $\left(C^{0} \cap \mathrm{PSH}\right)$-type such that

$$
0 \leq(E, h) \leq(D, g)
$$


Proof. Let $h_{1}$ be an $F_{\infty}$-invariant $E$-Green function of $\left(C^{\infty} \cap \mathrm{PSH}\right)$-type. There is a constant $C_{1}$ such that $h_{1}+C_{1} \leq g$ (a.e.). We set $h=\max \left\{h_{1}+C_{1}, 0\right\}$. Then, by Lemma 9.1.1, $h$ is an $F_{\infty}$-invariant $E$-Green function of ( $\left.C^{0} \cap \mathrm{PSH}\right)$-type and $0 \leq h \leq g$ (a.e.).

\section{§9.2. The existence of Zariski decompositions}

Let $\bar{D}=(D, g)$ be an arithmetic $\mathbb{R}$-Cartier divisor of $\mathscr{T}$-type on $X$ such that $g$ is of upper bounded type. Let us consider

$$
(-\infty, \bar{D}] \cap \widehat{\operatorname{Nef}}(X)_{\mathbb{R}}=\{\bar{M} \mid \bar{M} \text { is nef and } \bar{M} \leq \bar{D}\} .
$$

The following theorem is one of the main theorems of this paper, which guarantees the existence of the greatest element $\bar{P}$ of $(-\infty, \bar{D}] \cap \widehat{\mathrm{Nef}}(X)_{\mathbb{R}}$ under the assumption $(-\infty, \bar{D}] \cap \widehat{\operatorname{Nef}}(X)_{\mathbb{R}} \neq \emptyset$. If we set $\bar{N}=\bar{D}-\bar{P}$, then we have a decomposition $\bar{D}=\bar{P}+\bar{N}$. It is called the Zariski decomposition of $\bar{D}$, and $\bar{P}$ (resp. $\bar{N}$ ) is called the positive part (resp. negative part) of $\bar{D}$.

Theorem 9.2.1 (Zariski decomposition on an arithmetic surface). If

$$
(-\infty, \bar{D}] \cap \widehat{\operatorname{Nef}}(X)_{\mathbb{R}} \neq \emptyset,
$$

then there is $\bar{P}=(P, p) \in(-\infty, \bar{D}] \cap \widehat{\operatorname{Nef}}(X)_{\mathbb{R}}$ such that $\bar{P}$ is greatest in $(-\infty, \bar{D}] \cap$ $\widehat{\operatorname{Nef}}(X)_{\mathbb{R}}$, that is, $\bar{M} \leq \bar{P}$ for all $\bar{M} \in(-\infty, \bar{D}] \cap \widehat{\operatorname{Nef}}(X)_{\mathbb{R}}$. Moreover, if $\bar{D}$ is of $C^{0}$-type, then $\bar{P}$ is also of $C^{0}$-type.

Proof. For a 1-dimensional closed integral subscheme $C$ of $X$, we put

$$
a(C)=\sup \left\{\operatorname{mult}_{C}(M) \mid\left(M, g_{M}\right) \in(-\infty, \bar{D}] \cap \widehat{\operatorname{Nef}}(X)_{\mathbb{R}}\right\}
$$

We choose $\bar{M}_{0}=\left(M_{0}, g_{0}\right) \in(-\infty, \bar{D}] \cap \widehat{\operatorname{Nef}}(X)_{\mathbb{R}}$. Then $\operatorname{mult}_{C}\left(M_{0}\right) \leq a(C) \leq$ mult $_{C}(D)$. Let $\left\{C_{1}, \ldots, C_{l}\right\}$ be the set of all 1-dimensional closed integral subschemes in $\operatorname{Supp}(D) \cup \operatorname{Supp}\left(M_{0}\right)$. Note that if $C \notin\left\{C_{1}, \ldots, C_{l}\right\}$, then $a(C)=0$. We set $P=\sum_{C} a(C) C$.

Claim 9.2.1.1. There is a sequence $\left\{\bar{M}_{n}=\left(M_{n}, g_{n}\right)\right\}_{n=0}^{\infty}$ in $(-\infty, \bar{D}] \cap \widehat{\operatorname{Nef}}(X)_{\mathbb{R}}$ such that $\bar{M}_{n} \leq \bar{M}_{n+1}$ for all $n \geq 0$ and

$$
\lim _{n \rightarrow \infty} \operatorname{mult}_{C_{i}}\left(M_{n}\right)=a\left(C_{i}\right)
$$

for all $i=1, \ldots, n$.

Proof. For each $i$, let $\left\{\bar{M}_{i, n}\right\}_{n=1}^{\infty}$ be a sequence in $(-\infty, \bar{D}] \cap \widehat{\operatorname{Nef}}(X)_{\mathbb{R}}$ such that

$$
\lim _{n \rightarrow \infty} \operatorname{mult}_{C_{i}}\left(M_{i, n}\right)=a\left(C_{i}\right) .
$$


We set $\bar{M}_{n}=\max \left\{\left\{\bar{M}_{0}\right\} \cup\left\{\bar{M}_{i, j}\right\}_{1 \leq i \leq l, 1 \leq j \leq n}\right\}$ for $n \geq 1$. By Lemma 9.1.2, $\bar{M}_{n} \in(-\infty, \bar{D}] \cap \widehat{\operatorname{Nef}}(X)_{\mathbb{R}}$. Moreover, $\bar{M}_{n} \leq \bar{M}_{n+1}$ and

$$
\lim _{n \rightarrow \infty} \operatorname{mult}_{C_{i}}\left(M_{n}\right)=a\left(C_{i}\right)
$$

for all $i$

Since PSH is a subjacent type of $\mathscr{T}$, by Lemma 2.3 .1 we have

$$
\left(g_{0}\right)_{\mathrm{can}} \leq \cdots \leq\left(g_{n}\right)_{\mathrm{can}} \leq\left(g_{n+1}\right)_{\mathrm{can}} \leq \cdots \leq g_{\text {can }}
$$

on $X(\mathbb{C}) \backslash\left(\operatorname{Supp}(D) \cup \operatorname{Supp}\left(M_{0}\right)\right)(\mathbb{C})$, which means that $\lim _{n \rightarrow \infty}\left(g_{n}\right)_{\text {can }}(x)$ exists for $x \in X(\mathbb{C}) \backslash\left(\operatorname{Supp}(D) \cup \operatorname{Supp}\left(M_{0}\right)\right)(\mathbb{C})$. Therefore, by Theorem 7.1, there is an $F_{\infty}$-invariant $P$-Green function $h$ of $\mathrm{PSH}_{\mathbb{R}}$-type on $X(\mathbb{C})$ such that $(P, h) \leq \bar{D}$ and $(P, h)$ is nef. Now we consider

$$
[(P, h), \bar{D}] \cap \widehat{\operatorname{Nef}}(X)_{\mathbb{R}}=\left\{\left(M, g_{M}\right) \mid\left(M, g_{M}\right) \text { is nef and }(P, h) \leq\left(M, g_{M}\right) \leq \bar{D}\right\} \text {. }
$$

Note that $M=P$ for all $\left(M, g_{M}\right) \in[(P, h), \bar{D}] \cap \widehat{\operatorname{Nef}}(X)_{\mathbb{R}}$.

Claim 9.2.1.2. If $\bar{P}=(P, p)$ is the greatest element of $[(P, h), \bar{D}] \cap \widehat{\operatorname{Nef}}(X)_{\mathbb{R}}$, then $\bar{P}$ is also the greatest element of $(-\infty, \bar{D}] \cap \widehat{\operatorname{Nef}}(X)_{\mathbb{R}}$.

Proof. For $\left(N, g_{N}\right) \in(-\infty, \bar{D}] \cap \widehat{\operatorname{Nef}}(X)_{\mathbb{R}}$, we set

$$
\left(M, g_{M}\right)=\left(\max \{P, N\}, \max \left\{h, g_{N}\right\}\right) .
$$

Then

$$
\left(M, g_{M}\right) \in[(P, h), \bar{D}] \cap \widehat{\operatorname{Nef}}(X)_{\mathbb{R}} \quad \text { and } \quad\left(N, g_{N}\right) \leq\left(M, g_{M}\right) .
$$

Thus the claim follows.

By Proposition 4.4, there is a $P$-Green function $p$ of $\mathrm{PSH}_{\mathbb{R}}$-type such that $p \leq g$ (a.e.) and $p_{\text {can }}$ is the upper semicontinuous regularization of the function $p^{\prime}$ given by

$$
p^{\prime}(x):=\sup \left\{\left(g_{M}\right)_{\operatorname{can}}(x) \mid \bar{M} \in[(P, h), \bar{D}] \cap \widehat{\operatorname{Nef}}(X)_{\mathbb{R}}\right\}
$$

over $X(\mathbb{C}) \backslash \operatorname{Supp}(P)(\mathbb{C})$. Since $\left(g_{M}\right)_{\text {can }}$ is $F_{\infty}$-invariant on $X(\mathbb{C}) \backslash \operatorname{Supp}(P)(\mathbb{C})$, $p^{\prime}$ is also $F_{\infty}$-invariant, and hence $p$ is $F_{\infty}$-invariant because $p=p^{\prime}$ (a.e.) on $X(\mathbb{C}) \backslash \operatorname{Supp}(P)(\mathbb{C})$ (cf. Subsection 2.1). We set $\bar{P}=(P, p)$. Then $(P, h) \leq \bar{P} \leq \bar{D}$ and hence $\bar{P}$ is nef by Lemma 6.2.3. In addition, $\bar{P}$ is the greatest element of $[(P, h), \bar{D}] \cap \widehat{\operatorname{Nef}}(X)_{\mathbb{R}}$.

Finally, assume that $\bar{D}$ is of $C^{0}$-type. Let $e$ be the degree of $P$ on the generic fiber of $X \rightarrow \operatorname{Spec}(\mathbb{Z})$. As $\bar{P}$ is nef, we have $e \geq 0$. Let $X(\mathbb{C})=X_{1} \cup \cdots \cup X_{r}$ be the decomposition of $X(\mathbb{C})$ into connected components. We set $P=\sum_{i=1}^{r} \sum_{j} a_{i j} P_{i j}$ 
on $X(\mathbb{C})$, where $P_{i j} \in X_{i}$ for all $i$ and $j$. Note that $e=\sum_{j} a_{i j}$ for all $i$. Let us fix a $C^{\infty}$-volume form $\omega_{i}$ on $X_{i}$ with $\int_{X_{i}} \omega_{i}=1$. Let $p_{i j}$ be a $P_{i j}$-Green function of $C^{\infty}$-type on $X_{i}$ such that $d d^{c}\left(\left[p_{i j}\right]\right)+\delta_{P_{i j}}=\left[\omega_{i}\right]$. We set $p^{\prime}=\sum_{i=1}^{r} \sum_{j} a_{i j} p_{i j}$. Then $p^{\prime}$ is a $P$-Green function of $C^{\infty}$-type and

$$
d d^{c}\left(\left[p^{\prime}\right]\right)+\delta_{P}=\sum_{i=1}^{r}\left(\sum_{j} a_{i j}\right)\left[\omega_{i}\right]=e \sum_{i=1}^{r}\left[\omega_{i}\right] .
$$

Thus, if $e>0$, then $d d^{c}\left(\left[p^{\prime}\right]\right)+\delta_{P}$ is represented by a positive $C^{\infty}$-form $e \sum_{i=1}^{r} \omega_{i}$. Moreover, if $e=0$, then $d d^{c}\left(\left[p^{\prime}\right]\right)+\delta_{P}=0$. Let us consider the set

$$
\left\{\begin{array}{l|l}
\varphi & \begin{array}{l}
\varphi \text { is a } P \text {-Green function of PSH-type } \\
\text { on } X(\mathbb{C}) \text { with } \varphi \leq g \text { (a.e.) }
\end{array}
\end{array}\right\} .
$$

By Theorem 4.6, the above set has the greatest element $\tilde{p}$ modulo null functions such that $\tilde{p}$ is a $P$-Green function of $\left(C^{0} \cap \mathrm{PSH}\right)$-type. Since $g$ is $F_{\infty}$-invariant, we have $F_{\infty}^{*}(\tilde{p}) \leq F_{\infty}^{*}(g)=g$ (a.e.). Moreover, by Lemmas 5.1.1 and 5.1.2, $F_{\infty}^{*}(\tilde{p})$ is a $P$-Green function of PSH-type. Thus $F_{\infty}^{*}(\tilde{p}) \leq \tilde{p}$ (a.e.), and hence

$$
\tilde{p}=F_{\infty}^{*}\left(F_{\infty}^{*}(\tilde{p})\right) \leq F_{\infty}^{*}(\tilde{p}) \quad \text { (a.e.). }
$$

Therefore, $\tilde{p}$ is $F_{\infty}$-invariant. Note that $(P, \tilde{p})$ is nef because $p \leq \tilde{p}$ (a.e.). Hence $p=\tilde{p}$ (a.e.).

\section{§9.3. Properties of Zariski decompositions}

Let $\bar{D}=(D, g)$ be an arithmetic $\mathbb{R}$-Cartier divisor of $\mathscr{T}$-type on $X$ such that $g$ is of upper bounded type. First of all, let us record the following three properties of Zariski decompositions:

Proposition 9.3.1. Assume $(-\infty, \bar{D}] \cap \widehat{\operatorname{Nef}}(X)_{\mathbb{R}} \neq \emptyset$. Let $\bar{D}=\bar{P}+\bar{N}$ be the Zariski decomposition of $\bar{D}$. Then we have the following:

(1) For a non-zero rational function $\phi$ on $X, \bar{D}+\widehat{(\phi)}=(\bar{P}+\widehat{(\phi)})+\bar{N}$ is the Zariski decomposition of $\bar{D}+\widehat{(\phi)}$.

(2) For $a \in \mathbb{R}_{>0}, a \bar{D}=a \bar{P}+a \bar{N}$ is the Zariski decomposition of $a \bar{D}$.

Proof. Note that $\pm \widehat{(\phi)}$ is nef and

$$
\bar{D}_{1} \leq \bar{D}_{2} \Leftrightarrow \bar{D}_{1}+\widehat{(\phi)} \leq \bar{D}_{2}+\widehat{(\phi)}
$$

and

$$
\bar{D}_{1} \leq \bar{D}_{2} \Leftrightarrow a \bar{D}_{1} \leq a \bar{D}_{2}
$$

for arithmetic $\mathbb{R}$-Cartier divisors $\bar{D}_{1}, \bar{D}_{2}$, any non-zero rational function $\phi$ and $a \in \mathbb{R}_{>0}$. Thus the assertions of this proposition are obvious. 
Proposition 9.3.2. (1) If $\hat{h}^{0}(X, a \bar{D}) \neq 0$ for some $a \in \mathbb{R}_{>0}$, then

$$
(-\infty, \bar{D}] \cap \widehat{\operatorname{Nef}}(X)_{\mathbb{R}} \neq \emptyset
$$

(2) If $\bar{D}$ is of $C^{0}$-type and $(-\infty, \bar{D}] \cap \widehat{\operatorname{Nef}}(X)_{\mathbb{R}} \neq \emptyset$, then $\bar{D}$ is pseudo-effective.

Proof. (1) We choose $\phi \in \hat{H}^{0}(X, a \bar{D}) \backslash\{0\}$. Then $a \bar{D}+\widehat{(\phi)} \geq 0$, which implies $\bar{D} \geq$ $(-1 / a) \widehat{(\phi)}$. Note that $(-1 / a) \widehat{(\phi)}$ is nef, so that $(-1 / a) \widehat{(\phi)} \in(-\infty, \bar{D}] \cap \widehat{\operatorname{Nef}}(X)_{\mathbb{R}}$, as required.

(2) Let $\bar{D}=\bar{P}+\bar{N}$ be the Zariski decomposition of $\bar{D}$ and let $\bar{A}$ be an ample arithmetic $\mathbb{R}$-Cartier divisor. For $n \in \mathbb{Z}_{>0}$, by Proposition $6.2 .2, \bar{P}+(1 / n) \bar{A}$ is adequate. In particular, $\widehat{\operatorname{vol}}(\bar{P}+(1 / n) \bar{A})>0$, and hence

$$
\widehat{\operatorname{vol}}(\bar{D}+(1 / n) \bar{A}) \geq \widehat{\operatorname{vol}}(\bar{P}+(1 / n) \bar{A})>0,
$$

which shows that $\bar{D}$ is pseudo-effective.

Remark 9.3.3. It is expected that the converse of (2) in Proposition 9.3.2 holds, that is, if $\bar{D}$ is of $C^{0}$-type and $\bar{D}$ is pseudo-effective, then $(-\infty, \bar{D}] \cap \widehat{\operatorname{Nef}}(X)_{\mathbb{R}} \neq \emptyset$ (cf. [18]).

Proposition 9.3.4. Assume that $\bar{D}$ is of $C^{\infty}$-type and $\bar{D}$ is effective. Let $\bar{P}$ be the positive part of the Zariski decomposition of $\bar{D}$. Then there is a constant $e^{\prime}$ such that

$$
\hat{h}^{0}(X, n \bar{P}) \leq \hat{h}^{0}(X, n \bar{D}) \leq \hat{h}^{0}(X, n \bar{P})+e^{\prime} n \log (n+1)
$$

for all $n \geq 1$. In particular, $\widehat{\operatorname{vol}}(\bar{P})=\widehat{\operatorname{vol}}(\bar{D})$.

Proof. The assertion is a consequence of Proposition 8.3 because $\bar{M}_{\infty}(\bar{D}) \leq \bar{P}$.

The following theorem is also one of the main theorems of this paper.

Theorem 9.3.5. Assume that $\bar{D}$ is of $C^{0}$-type and $(-\infty, \bar{D}] \cap \widehat{\operatorname{Nef}}(X)_{\mathbb{R}} \neq \emptyset$. Let $\bar{P}($ resp. $\bar{N})$ be the positive part (resp. negative part) of the Zariski decomposition of $\bar{D}$. Then we have the following:

(1) $\widehat{\operatorname{vol}}(\bar{P})=\widehat{\operatorname{vol}}(\bar{D})=\widehat{\operatorname{deg}}\left(\bar{P}^{2}\right)$.

(2) $\widehat{\operatorname{deg}}\left(\left.\bar{P}\right|_{C}\right)=0$ for all 1-dimensional closed integral subschemes $C$ with $C \subseteq$ $\operatorname{Supp}(N)$.

(3) If $\bar{M}$ is an arithmetic $\mathbb{R}$-Cartier divisor of $\mathrm{PSH}_{\mathbb{R}}$-type on $X$ such that $0 \leq$ $\bar{M} \leq \bar{N}$ and $\operatorname{deg}\left(\left.\bar{M}\right|_{C}\right) \geq 0$ for all 1-dimensional closed integral subschemes $C$ with $C \subseteq \operatorname{Supp}(N)$, then $\bar{M}=0$.

(4) Assume $N \neq 0$. Let $N=c_{1} C_{1}+\cdots+c_{l} C_{l}$ be a decomposition such that $c_{1}, \ldots, c_{l} \in \mathbb{R}_{>0}$ and $C_{1}, \ldots, C_{l}$ are distinct 1 -dimensional closed integral subschemes on $X$. Then the following hold: 
(4.1) There are effective arithmetic Cartier divisors $\left(C_{1}, h_{1}\right), \ldots,\left(C_{l}, h_{l}\right)$ of $\left(C^{0} \cap \mathrm{PSH}\right)$-type such that $c_{1}\left(C_{1}, h_{1}\right)+\cdots+c_{l}\left(C_{l}, h_{l}\right) \leq \bar{N}$.

(4.2) If $\left(C_{1}, k_{1}\right), \ldots,\left(C_{l}, k_{l}\right)$ are effective arithmetic Cartier divisors of $\mathrm{PSH}_{\mathbb{R}^{-}}$ type such that $\alpha_{1}\left(C_{1}, k_{1}\right)+\cdots+\alpha_{l}\left(C_{l}, k_{l}\right) \leq \bar{N}$ for some $\alpha_{1}, \ldots, \alpha_{l} \in$ $\mathbb{R}_{>0}$, then

$$
(-1)^{l} \operatorname{det}\left(\widehat{\operatorname{deg}}\left(\left.\left(C_{i}, k_{i}\right)\right|_{C_{j}}\right)\right)>0 \text {. }
$$

Proof. (1) It follows from Proposition 6.4.2 that $\widehat{\operatorname{vol}}(\bar{P})=\widehat{\operatorname{deg}}\left(\bar{P}^{2}\right)$. We need to show $\widehat{\operatorname{vol}}(\bar{P})=\widehat{\operatorname{vol}}(\bar{D})$. If $\widehat{\operatorname{vol}}(\bar{D})=0$, then the assertion is obvious, so that we may assume that $\widehat{\operatorname{vol}}(\bar{D})>0$.

First we consider the case where $\bar{D}$ is of $C^{\infty}$-type. We choose a positive integer $n$ and a non-zero rational function $\phi$ such that $n \bar{D}+\widehat{(\phi)}$ is effective. By Proposition 9.3.1, the positive part of the Zariski decomposition $n \bar{D}+\widehat{(\phi)}$ is $n \bar{P}+\widehat{(\phi)}$. Thus, by Proposition 9.3.4,

$$
n^{2} \widehat{\operatorname{vol}}(\bar{P})=\widehat{\operatorname{vol}}(n \bar{P})=\widehat{\operatorname{vol}}(n \bar{P}+\widehat{(\phi)})=\widehat{\operatorname{vol}}(n \bar{D}+\widehat{(\phi)})=\widehat{\operatorname{vol}}(n \bar{D})=n^{2} \widehat{\operatorname{vol}}(\bar{D}),
$$

as required.

Let us consider the general case. By the Stone-Weierstrass theorem, there is a sequence $\left\{u_{n}\right\}_{n=1}^{\infty}$ of non-negative $F_{\infty}$-invariant continuous functions such that $\lim _{n \rightarrow \infty}\left\|u_{n}\right\|_{\text {sup }}=0$ and $\bar{D}_{n}:=\bar{D}-\left(0, u_{n}\right)$ is of $C^{\infty}$-type for every $n \geq 1$. By the continuity of $\widehat{\text { vol }}$ (cf. Theorem 5.2.2),

$$
\lim _{n \rightarrow \infty} \widehat{\operatorname{vol}}\left(\bar{D}_{n}\right)=\widehat{\operatorname{vol}}(\bar{D})
$$

In particular, $\bar{D}_{n}$ is big for $n \gg 1$. Let $\bar{P}_{n}$ be the positive part of the Zariski decomposition of $\bar{D}_{n}$. Since $\bar{P}_{n} \leq \bar{D}_{n} \leq \bar{D}$ and $\bar{P}_{n}$ is nef, we have $\bar{P}_{n} \leq \bar{P}$, and hence

$$
\widehat{\operatorname{vol}}\left(\bar{D}_{n}\right)=\widehat{\operatorname{vol}}\left(\bar{P}_{n}\right) \leq \widehat{\operatorname{vol}}(\bar{P}) \leq \widehat{\operatorname{vol}}(\bar{D})
$$

Thus the assertion follows by letting $n \rightarrow \infty$.

(4.1) Let us check (4.1) before starting the proofs of (2) and (3). By Proposition 2.4.2, there are effective arithmetic Cartier divisors $\left(C_{1}, h_{1}^{\prime}\right), \ldots,\left(C_{l}, h_{l}^{\prime}\right)$ of $C^{0}$ type such that $c_{1}\left(C_{1}, h_{1}^{\prime}\right)+\cdots+c_{l}\left(C_{l}, h_{l}^{\prime}\right)=\bar{N}$. For each $i$, by using Lemma 9.1.3, we can find an effective arithmetic Cartier divisor $\left(C_{i}, h_{i}\right)$ of $\left(C^{0} \cap \mathrm{PSH}\right)$-type such that $\left(C_{i}, h_{i}\right) \leq\left(C_{i}, h_{i}^{\prime}\right)$, as required.

(2) We may assume $N \neq 0$. We assume $\operatorname{deg}\left(\left.\bar{P}\right|_{C_{i}}\right)>0$ for some $i$. By (4.1),

$$
0 \leq c_{i}\left(C_{i}, h_{i}\right) \leq \bar{N}
$$

Note that if $C^{\prime}$ is a 1-dimensional closed integral subscheme with $C^{\prime} \neq C_{i}$, then

$$
\operatorname{deg}\left(\left.\left(C_{i}, h_{i}\right)\right|_{C^{\prime}}\right) \geq 0 .
$$


Thus, since $\operatorname{deg}\left(\left.\bar{P}\right|_{C_{i}}\right)>0$, we can find a sufficiently small positive number $\epsilon$ such that $\bar{P}+\epsilon\left(C_{i}, h_{i}\right)$ is nef and $\bar{P}+\epsilon\left(C_{i}, h_{i}\right) \leq \bar{D}$. This is a contradiction.

(3) Since $0 \leq \bar{M} \leq \bar{N}$, if $C^{\prime}$ is a 1-dimensional closed integral subscheme with $C^{\prime} \nsubseteq \operatorname{Supp}(N)$, then $\operatorname{\operatorname {deg}}\left(\left.\bar{M}\right|_{C^{\prime}}\right) \geq 0$. Thus $\bar{M}$ is nef, and hence $\bar{P}+\bar{M}$ is nef and $\bar{P}+\bar{M} \leq \bar{D}$. Therefore, $\bar{M}=0$.

(4.2) By Lemma 1.2.3, it is sufficient to prove the following: if $\beta_{1}, \ldots, \beta_{l} \in \mathbb{R}_{\geq 0}$ and

$$
\widehat{\operatorname{deg}}\left(\left.\left(\beta_{1}\left(C_{1}, k_{1}\right)+\cdots+\beta_{l}\left(C_{l}, k_{l}\right)\right)\right|_{C_{i}}\right) \geq 0
$$

for all $i$, then $\beta_{1}=\cdots=\beta_{l}=0$. Replacing $\beta_{1}, \ldots, \beta_{l}$ with $t \beta_{1}, \ldots, t \beta_{l}(t>0)$, we may assume that $0 \leq \beta_{i} \leq \alpha_{i}$ for all $i$. Thus the assertion follows from (3).

Theorem 9.3.6 (Asymptotic orthogonality of $\sigma$-decomposition). If $\bar{D}$ is of $C^{0}$ type, effective and big, then

$$
\lim _{n \rightarrow \infty} \widehat{\operatorname{deg}}\left(\bar{M}_{n}(\bar{D}) \mid F_{n}(\bar{D})\right)=0 .
$$

(For the definition of $\bar{M}_{n}(\bar{D})$ and $F_{n}(\bar{D})$, see Section 8.)

Proof. Let us begin with the following claim:

Claim 9.3.6.1. $P=M_{\infty}(\bar{D})$ and $N=F_{\infty}(\bar{D})$.

Proof. First of all, note that $\bar{M}_{\infty}(\bar{D}) \leq \bar{P}$ and $\bar{F}_{\infty}(\bar{D}) \geq \bar{N}$. Since $\bar{D}$ is effective, $(0,0) \in(-\infty, \bar{D}] \cap \widehat{\operatorname{Nef}}(X)_{\mathbb{R}}$, so that $\bar{P}$ is effective. Then, by Proposition 6.5.2(2),

$$
\mu_{C}(\bar{D}) \leq \mu_{C}(\bar{P})+\operatorname{mult}_{C}(N) .
$$

Moreover, by Proposition 6.5.3, $\mu_{C}(\bar{P})=0$ because $\bar{P}$ is nef and big. Thus we have

$$
\operatorname{mult}_{C}\left(F_{\infty}(\bar{D})\right)=\mu_{C}(\bar{D}) \leq \operatorname{mult}_{C}(N),
$$

which implies $F_{\infty}(\bar{D}) \leq N$. Therefore, $N=F_{\infty}(\bar{D})$, and hence $P=M_{\infty}(\bar{D})$.

Claim 9.3.6.2. $\widehat{\operatorname{deg}}\left(\left.\bar{M}_{\infty}(\bar{D})\right|_{C}\right)=0$ for any 1-dimensional closed integral subscheme $C$ with $C \subseteq \operatorname{Supp}(N)$.

Proof. Since $\bar{M}_{\infty}(\bar{D}) \leq \bar{P}$ and $P=M_{\infty}(\bar{D})$, there is $\phi \in\left(C^{0}-\mathrm{PSH}_{\mathbb{R}}\right)(X(\mathbb{C}))$ such that $\phi \geq 0$ and $\bar{P}=\bar{M}_{\infty}(\bar{D})+(0, \phi)$. Thus, for a 1-dimensional closed integral subscheme $C$ with $C \subseteq \operatorname{Supp}(N)$, by Theorem 9.3.5(3),

$$
0 \leq \widehat{\operatorname{deg}}\left(\left.\bar{M}_{\infty}(\bar{D})\right|_{C}\right) \leq \widehat{\operatorname{deg}}\left(\left.\bar{P}\right|_{C}\right)=0 .
$$

Let $C_{1}, \ldots, C_{l}$ be irreducible components of $\operatorname{Supp}(D)$. We set $F_{n}(\bar{D})=$ $\sum_{i=1}^{l} a_{n i} C_{i}$ and $F_{\infty}(\bar{D})=\sum_{i=1}^{l} a_{i} C_{i}$. Then $\lim _{n \rightarrow \infty} a_{n i}=a_{i}$. Moreover, if we 
set $I=\left\{i \mid a_{i}>0\right\}$, then $\bigcup_{i \in I} C_{i}=\operatorname{Supp}(N)$. Therefore, by the above claim and Proposition 8.3(3),

$$
\begin{aligned}
0 & \leq \liminf _{n \rightarrow \infty} \widehat{\operatorname{deg}}\left(\bar{M}_{n}(\bar{D}) \mid F_{n}(\bar{D})\right) \leq \limsup _{n \rightarrow \infty} \widehat{\operatorname{deg}}\left(\bar{M}_{n}(\bar{D}) \mid F_{n}(\bar{D})\right) \\
& \leq \sum_{i=1}^{l} \limsup _{n \rightarrow \infty} a_{n i} \widehat{\operatorname{deg}}\left(\left.\bar{M}_{n}(\bar{D})\right|_{C_{i}}\right)=\sum_{i=1}^{l} a_{i} \limsup _{n \rightarrow \infty} \widehat{\operatorname{deg}}\left(\left.\bar{M}_{n}(\bar{D})\right|_{C_{i}}\right) \\
& =\sum_{i \in I} a_{i} \limsup _{n \rightarrow \infty} \widehat{\operatorname{deg}}\left(\left.\bar{M}_{n}(\bar{D})\right|_{C_{i}}\right) \leq \sum_{i \in I} a_{i} \widehat{\operatorname{deg}}\left(\left.\bar{M}_{\infty}(\bar{D})\right|_{C_{i}}\right)=0 .
\end{aligned}
$$

Finally let us consider Fujita's approximation theorem on an arithmetic surface.

Proposition 9.3.7. Assume that $\bar{D}$ is $C^{0}$-type and $\widehat{\operatorname{vol}}(\bar{D})>0$. Then, for any $\epsilon>0$, there is $\bar{A} \in \widehat{\operatorname{Div}}_{C}(X)_{\mathbb{R}}$ such that

$$
\bar{A} \text { is nef, } \quad \bar{A} \leq \bar{D} \quad \text { and } \widehat{\operatorname{vol}}(\bar{A}) \geq \widehat{\operatorname{vol}}(\bar{D})-\epsilon
$$

Proof. By using the continuity of $\widehat{\text { vol}}$, we can find a sufficiently small positive number $\delta$ such that

$$
\widehat{\operatorname{vol}}(\bar{D}-(0, \delta))>\max \{\widehat{\operatorname{vol}}(\bar{D})-\epsilon, 0\} .
$$

Let $\bar{D}-(0, \delta)=\bar{P}_{\delta}+\bar{N}_{\delta}$ be the Zariski decomposition of $\bar{D}-(0, \delta)$. Since $\bar{P}_{\delta}$ is a big arithmetic $\mathbb{R}$-Cartier divisor of $C^{0}$-type, by Theorem 4.6 there is an $F_{\infty}$-invariant continuous function $u$ on $X(\mathbb{C})$ such that $0 \leq u<\delta$ on $X(\mathbb{C})$ and $\bar{P}_{\delta}+(0, u)$ is nef and of $C^{\infty}$-type. If we set $\bar{A}=\bar{P}_{\delta}+(0, u)$, then $\bar{A} \leq \bar{D}$ and

$$
\widehat{\operatorname{vol}}(\bar{D})-\epsilon<\widehat{\operatorname{vol}}(\bar{D}-(0, \delta)) \leq \widehat{\operatorname{vol}}(\bar{A}) .
$$

Remark 9.3.8. Assume that $\bar{D}$ is of $C^{0}$-type, big and not nef. Let $\bar{D}=\bar{P}+\bar{N}$ be the Zariski decomposition of $\bar{D}$ and let $N=c_{1} C_{1}+\cdots+c_{l} C_{l}$ be the decomposition such that $c_{1}, \ldots, c_{l} \in \mathbb{R}_{>0}$ and $C_{1}, \ldots, C_{l}$ are distinct 1-dimensional closed integral subschemes on $X$. Then $C_{1}, \ldots, C_{l}$ are not necessarily linearly independent in $\operatorname{Pic}(X) \otimes_{\mathbb{Z}} \mathbb{Q}($ cf. Remark 9.4.2).

Remark 9.3.9. After this paper was completed, several significant advances have been made in the study of Zariski decompositions. Here we report on them briefly. Let $\bar{D}$ and $\bar{P}$ be arithmetic $\mathbb{R}$-Cartier divisors of $C^{0}$-type on $X$.

(1) A generalization of Proposition 9.3.4 was found: if $\bar{P}$ is the greatest element of $(-\infty, \bar{D}] \cap \widehat{\operatorname{Nef}}_{C^{0}}(X)_{\mathbb{R}}$, then $\hat{h}^{0}(X, n \bar{P})=\hat{h}^{0}(X, n \bar{D})$ for all $n \geq 0$ (cf. [20, Appendix B]). This can be proved as follows: If $\phi \in \hat{H}^{0}(X, n \bar{D}) \backslash\{0\}$, then $(-1 / n) \widehat{(\phi)} \in(-\infty, \bar{D}] \cap \widehat{\operatorname{Nef}}_{C^{0}}(X)_{\mathbb{R}}$ because $n \bar{D}+\widehat{(\phi)} \geq 0$ and $-\widehat{(\phi)}$ is nef. Thus $(-1 / n) \widehat{(\phi)} \leq \bar{P}$, and hence $\phi \in \hat{H}^{0}(X, n \bar{P})$. 
(2) A numerical characterization of the greatest element of the set $(-\infty, \bar{D}] \cap$ $\widehat{\mathrm{Nef}}_{C^{0}}(X)_{\mathbb{R}}$ was obtained, by proving that the following are equivalent (cf. [20]):

(a) $\bar{P}$ is the greatest element of $(-\infty, \bar{D}] \cap \widehat{\operatorname{Nef}}_{C^{0}}(X)_{\mathbb{R}}$.

(b) $\bar{P}$ is an element of $(-\infty, \bar{D}] \cap \widehat{\operatorname{Nef}}_{C^{0}}(X)_{\mathbb{R}}$ with the following property: if $\bar{B}$ is an arithmetic $\mathbb{R}$-Cartier divisor of $C^{0}$-type such that $(0,0) \supsetneqq \bar{B} \leq \bar{D}-\bar{P}$ and $\bar{P}+\bar{B}$ is of $\left(C^{0} \cap \mathrm{PSH}\right)$-type, then $\widehat{\operatorname{deg}}(\bar{P} \cdot \bar{B})=0$ and $\widehat{\operatorname{deg}}\left(\bar{B}^{2}\right)<0$.

(3) In the case where $\bar{D}$ is big, the greatest element of $(-\infty, \bar{D}] \cap \widehat{\operatorname{Nef}}_{C^{0}}(X)_{\mathbb{R}}$ is characterized by $\widehat{\operatorname{vol}}(\bar{D})=\widehat{\operatorname{vol}}(\bar{P})$. Namely, if $\bar{D}$ is big, $\bar{P} \in(-\infty, \bar{D}] \cap \widehat{\operatorname{Nef}}_{C^{0}}(X)_{\mathbb{R}}$ and $\widehat{\operatorname{vol}}(\bar{D})=\widehat{\operatorname{vol}}(\bar{P})$, then $\bar{P}$ is the greatest element of $(-\infty, \bar{D}] \cap \widehat{\operatorname{Nef}}_{C^{0}}(X)_{\mathbb{R}}(\mathrm{cf}$. $[19$, Theorem 4.2.1]).

\section{§9.4. Examples of Zariski decompositions on $\mathbb{P}_{\mathbb{Z}}^{1}$}

Let $\mathbb{P}_{\mathbb{Z}}^{1}=\operatorname{Proj}(\mathbb{Z}[x, y]), C_{0}=\{x=0\}, C_{\infty}=\{y=0\}$ and $z=x / y$. Let $\alpha$ and $\beta$ be positive real numbers. We set

$$
D=C_{0}, \quad g=-\log |z|^{2}+\log \max \left\{\alpha^{2}|z|^{2}, \beta^{2}\right\} \quad \text { and } \quad \bar{D}=(D, g) .
$$

The purpose of this subsection is to show the following fact:

Proposition 9.4.1. The Zariski decomposition of $\bar{D}$ exists if and only if either $\alpha \geq 1$ or $\beta \geq 1$. Moreover, we have the following:

(1) If $\alpha \geq 1$ and $\beta \geq 1$, then $\bar{D}$ is nef.

(2) If $\alpha \geq 1$ and $\beta<1$, then the positive part of $\bar{D}$ is given by

$$
\left(\theta C_{0},-\theta \log |z|^{2}+\log \max \left\{\alpha^{2}|z|^{2 \theta}, 1\right\}\right),
$$

where $\theta=\log \alpha /(\log \alpha-\log \beta)$.

(3) If $\alpha<1$ and $\beta \geq 1$, then the positive part of $\bar{D}$ is given by

$$
\left(C_{0}-\left(1-\theta^{\prime}\right) C_{\infty},-\log |z|^{2}+\log \max \left\{|z|^{2 \theta^{\prime}}, \beta^{2}\right\}\right),
$$

where $\theta^{\prime}=\log \beta /(\log \beta-\log \alpha)$.

Proof. Let us begin with the following claim:

Claim 9.4.1.1. For $a, b, \lambda \in \mathbb{R}_{>0}$, set

$$
L=\lambda C_{0}, \quad h=-\lambda \log |z|^{2}+\log \max \left\{a^{2}|z|^{2 \lambda}, b^{2}\right\} \quad \text { and } \quad \bar{L}=(L, h) .
$$

Then we have the following:

(a) $\bar{L}$ is an arithmetic $\mathbb{R}$-Cartier divisor of $\left(C^{0} \cap \mathrm{PSH}\right)$-type. In addition, $\bar{L}$ is effective if and only if $a \geq 1$. 
(b) $H^{0}\left(\mathbb{P}_{\mathbb{Z}}^{1}, L\right)=\bigoplus_{i \in \mathbb{Z}, 0 \leq i \leq \lambda} \mathbb{Z} z^{-i}$.

(c) For $i \in \mathbb{Z}$ with $0 \leq i \leq \lambda$, $\left\|z^{-i}\right\|_{h}=\frac{1}{a^{1-i / \lambda} b^{i / \lambda}}$.

(d) For $s=\sum_{0 \leq i \leq \lambda} c_{i} z^{-i} \in H^{0}\left(\mathbb{P}_{\mathbb{Z}}^{1}, L\right)$,

$$
\|s\|_{h} \geq \sqrt{\sum_{0 \leq i \leq \lambda}\left(\frac{c_{i}}{a^{1-i / \lambda} b^{i / \lambda}}\right)^{2}} .
$$

(e) $\hat{H}^{0}\left(\mathbb{P}_{\mathbb{Z}}^{1}, \bar{L}\right)=\{0\}$ if $a<1$ and $b<1$.

(f) $\bar{L}$ is nef if and only if $a \geq 1$ and $b \geq 1$.

(g) $\bar{L}$ is adequate if $a^{2}>2^{\lambda}$ and $b^{2}>2^{\lambda}$.

Proof. (a) and (b) are obvious. (c) is a straightforward calculation. (e) follows from (d). We will prove (d), (f) and (g).

(d) Indeed,

$$
\begin{aligned}
\|s\|_{h} & \geq \sup _{|\zeta|=(b / a)^{1 / \lambda}}|s|_{h}(\zeta)=\frac{1}{a} \sup _{|\zeta|=(b / a)^{1 / \lambda}}\left|\sum_{0 \leq i \leq \lambda} c_{i} \zeta^{-i}\right| \\
& \geq \frac{1}{a} \sqrt{\int_{0}^{1}\left|\sum_{0 \leq i \leq \lambda} c_{i}\left((b / a)^{1 / \lambda} \exp (2 \pi \sqrt{-1} t)\right)^{-i}\right|^{2} d t} \\
& =\frac{1}{a} \sqrt{\sum_{0 \leq i, j \leq \lambda} \int_{0}^{1} c_{i} c_{j}(b / a)^{\frac{-i-j}{\lambda}} \exp (2 \pi \sqrt{-1}(j-i) t) d t} \\
& =\sqrt{\sum_{0 \leq i \leq \lambda}\left(\frac{c_{i}}{a^{1-i / \lambda} b^{i / \lambda}}\right)^{2}} .
\end{aligned}
$$

(f) It is easy to see that $\widehat{\operatorname{deg}}\left(\left.\bar{L}\right|_{C_{0}}\right)=\log b$ and $\widehat{\operatorname{deg}}\left(\left.\bar{L}\right|_{C_{\infty}}\right)=\log a$. For $\gamma \in \overline{\mathbb{Q}}$, let $C_{\gamma}$ be the 1-dimensional closed integral subscheme of $\mathbb{P}_{\mathbb{Z}}^{1}$ given by the Zariski closure of $\{(\gamma: 1)\}$. Then

$$
\widehat{\operatorname{deg}}\left(\left.\bar{L}\right|_{C_{\gamma}}\right) \geq \sum_{\sigma \in C_{\gamma}(\mathbb{C})}\left(-\lambda \log |\sigma(\gamma)|+\log \max \left\{a|\sigma(\gamma)|^{\lambda}, b\right\}\right) .
$$

Thus (f) follows.

(g) We choose $\delta \in \mathbb{R}_{>0}$ such that $a^{2} \geq(2(1+\delta))^{\lambda}$ and $b^{2} \geq(2(1+\delta))^{\lambda}$. Then, as

$$
\begin{aligned}
\lambda \log \left((1+\delta)|z|^{2}+(1+\delta)\right) \leq \lambda \log \max \left\{2(1+\delta)|z|^{2}\right. & , 2(1+\delta)\} \\
& \leq \log \max \left\{a^{2}|z|^{2 \lambda}, b^{2}\right\}
\end{aligned}
$$


we have

$$
\lambda\left(C_{0},-\log |z|^{2}+\log \left((1+\delta)|z|^{2}+(1+\delta)\right)\right) \leq \bar{L} .
$$

Note that $\left(C_{0},-\log |z|^{2}+\log \left((1+\delta)|z|^{2}+(1+\delta)\right)\right)$ is ample. Thus $(\mathrm{g})$ follows.

Next we claim the following:

Claim 9.4.1.2. If $\alpha<1$ and $\beta<1$, then the Zariski decomposition of $\bar{D}$ does not exist.

Proof. For $t>0$, we set

$$
\bar{D}_{t}=\left(C_{0},-\log |z|^{2}+\log \max \left\{t^{2} \alpha^{2}|z|^{2}, t^{2} \beta^{2}\right\}\right) .
$$

It is easy to see that

$$
a \bar{D}_{t_{1}}+b \bar{D}_{t_{2}}=(a+b) \bar{D}_{\left(t_{1}^{a} t_{2}^{b}\right)^{\frac{1}{a+b}}}
$$

for $t_{1}, t_{2} \in \mathbb{R}_{>0}$ and $a, b \in \mathbb{R}_{>0}$. Moreover, by Claim 9.4.1.1(g), $\bar{D}_{t_{0}}$ is adequate if $t_{0} \gg 1$. Assume that the Zariski decomposition of $\bar{D}$ exists. Let $\bar{P}$ be the positive part of $\bar{D}$. We choose $\epsilon>0$ such that $t_{0}^{\frac{\epsilon}{1+\epsilon}} \alpha<1$ and $t_{0}^{\frac{\epsilon}{1+\epsilon}} \beta<1$. Then $\bar{P}+\epsilon \bar{D}_{t_{0}}$ is adequate by Proposition 6.2.2. Thus, by Proposition 6.2.1,

$$
\widehat{\operatorname{vol}}\left(\bar{D}_{t_{0}^{\frac{\epsilon}{1+\epsilon}}}\right)=\frac{\widehat{\operatorname{vol}}\left((1+\epsilon) \bar{D}_{\left(t_{0}^{\epsilon}\right)^{\frac{1}{1+\epsilon}}}\right)}{(1+\epsilon)^{2}}=\frac{\widehat{\operatorname{vol}}\left(\bar{D}+\epsilon \bar{D}_{t_{0}}\right)}{(1+\epsilon)^{2}} \geq \frac{\widehat{\operatorname{vol}}\left(\bar{P}+\epsilon \bar{D}_{t_{0}}\right)}{(1+\epsilon)^{2}}>0
$$

which yields a contradiction by Claim 9.4.1.1(e).

By the above claim, it is sufficient to prove (1)-(3). (1) follows from (f) in Claim 9.4.1.1.

(2) In this case, $\bar{D}$ is effective. Thus the Zariski decomposition of $\bar{D}$ exists. First we assume that $\alpha>1$, so that $0<\theta<1$ and $\alpha^{1-\theta} \beta^{\theta}=1$. Let us prove the following claim:

Claim 9.4.1.3. $\left\langle\hat{H}^{0}\left(\mathbb{P}_{\mathbb{Z}}^{1}, n \bar{D}\right)\right\rangle_{\mathbb{Z}}=\bigoplus_{i \in \mathbb{Z}, 0 \leq i \leq n \theta} \mathbb{Z} z^{-i}$.

Proof. By Claim 9.4.1.1(c), $\left\|z^{-i}\right\|_{n g}=\beta^{\frac{n \theta-i}{1-\theta}}$. Thus $z^{-i} \in \hat{H}^{0}\left(\mathbb{P}_{\mathbb{Z}}^{1}, n \bar{D}\right)$ for $0 \leq i \leq$ $n \theta$. For $s=\sum_{i=0}^{n} a_{i} z^{-i} \in H^{0}\left(\mathbb{P}_{\mathbb{Z}}^{1}, n D\right)$, by Claim 9.4.1.1(d),

$$
\|s\|_{n g} \geq \sqrt{\sum_{i=0}^{n}\left(\left|a_{i}\right| \beta^{\frac{n \theta-i}{1-\theta}}\right)^{2}}
$$

Thus, if $\|s\|_{n g} \leq 1$, then $a_{i}=0$ for $i>n \theta$, which means $s \in \bigoplus_{0 \leq i \leq n \theta} \mathbb{Z} z^{-i}$. 
Claim 9.4.1.4. $\bar{D}$ is big and

$$
\mu_{C}(\bar{D})= \begin{cases}1-\theta & \text { if } C=C_{0}, \\ 0 & \text { if } C \neq C_{0}\end{cases}
$$

for a 1-dimensional closed integral subscheme $C$ of $\mathbb{P}_{\mathbb{Z}}^{1}$.

Proof. Note that $\left(z^{-i}\right)+n D=(n-i) C_{0}+i C_{\infty}$. Thus the second assertion follows from Claim 9.4.1.3. To show that $\bar{D}$ is big, we set

$$
S_{n}=\left\{\sum_{0 \leq i \leq n \theta / 3} a_{i} z^{-i}|| a_{i} \mid \leq \beta^{\frac{-i}{1-\theta}}\right\} .
$$

It is easy to see that $S_{n} \subseteq \hat{H}^{0}\left(\mathbb{P}_{\mathbb{Z}}^{1}, n \bar{D}\right)$ for $n \gg 1$. Note that, for $M \in \mathbb{R}_{\geq 0}$,

$$
\#\{a \in \mathbb{Z}|| a \mid \leq M\}=2\lfloor M\rfloor+1 \geq\lfloor M\rfloor+1 \geq M .
$$

Therefore

$$
\#\left(S_{n}\right) \geq \prod_{0 \leq i \leq n \theta / 3} \beta^{\frac{-i}{1-\theta}}=\beta^{\frac{-1}{1-\theta} \frac{\lfloor n \theta / 3\rfloor(\lfloor n \theta / 3\rfloor+1)}{2}}
$$

which implies

$$
\hat{h}^{0}(X, n \bar{D}) \geq \log \#\left(S_{n}\right) \geq \frac{-\log \beta}{1-\theta} \frac{\lfloor n \theta / 3\rfloor(\lfloor n \theta / 3\rfloor+1)}{2}
$$

for $n \gg 1$, and hence $\widehat{\operatorname{vol}}(\bar{D})>0$.

We set

$$
P^{\prime}=\theta C_{0}, \quad p^{\prime}=-\theta \log |z|^{2}+\log \max \left\{\alpha^{2}|z|^{2 \theta}, 1\right\} \quad \text { and } \quad \bar{P}^{\prime}=\left(P^{\prime}, p^{\prime}\right) .
$$

By Claims 9.4.1.4 and 9.3.6.1, if $\bar{P}=(P, p)$ is the positive part of the Zariski decomposition, then $P=\theta C_{0}$. Let us show that $\bar{P}^{\prime}=\bar{P}$. First of all, $\bar{P}^{\prime} \leq \bar{D}$ and $\bar{P}^{\prime}$ is nef by Claim 9.4.1.1(f). Thus $\bar{P}^{\prime} \leq \bar{P}$, and hence there is a continuous function $u$ such that $u \geq 0$ and $\bar{P}=\bar{P}^{\prime}+(0, u)$. Note that

$$
0 \leq u \leq-(1-\theta) \log |z|^{2}+\log \max \left\{\alpha^{2}|z|^{2}, \beta^{2}\right\}-\log \max \left\{\alpha^{2}|z|^{2 \theta}, 1\right\}
$$

In particular, if $|z| \geq \beta^{\frac{1}{1-\theta}}$, then $u(z)=0$. As $p=-\theta \log |z|^{2}+u$ on $\{z|| z \mid<$ $\left.\beta^{\frac{1}{1-\theta}}\right\}, u$ is subharmonic on $\left\{z|| z \mid<\beta^{\frac{1}{1-\theta}}\right\}$. Thus, by the maximal principle,

$$
u(z) \leq \sup _{|\zeta|=\beta^{1-\theta}} u(\zeta)=0,
$$

which implies that $u(z)=0$ on $\left\{z|| z \mid<\beta^{\frac{1}{1-\theta}}\right\}$. Therefore $\bar{P}^{\prime}=\bar{P}$. 
Finally let us consider the case where $\alpha=1$. Let $\bar{P}$ be the positive part of $\bar{D}$. For $t \in(1,1 / \beta)$, we set

$$
\bar{D}_{t}=\left(C_{0},-\log |z|^{2}+\log \max \left\{t^{2}|z|^{2}, t^{2} \beta^{2}\right\}\right)
$$

as in the proof of Claim 9.4.1.2. Then $\bar{D} \leq \bar{D}_{t}$ and, by the previous observation, the positive part $\bar{P}_{t}$ of $\bar{D}_{t}$ is given by

$$
\bar{P}_{t}=\left(\theta_{t} C_{0},-\theta_{t} \log |z|^{2}+\log \max \left\{t^{2}|z|^{2 \theta_{t}}, 1\right\}\right),
$$

where $\theta_{t}=\log t /(-\log \beta)$. Therefore, $(0,0) \leq \bar{P} \leq \bar{P}_{t}$, and hence $\bar{P}=(0,0)$ as $t \rightarrow 1$.

(3) If we set $\bar{D}^{\prime \prime}=\bar{D}-\widehat{(z)}$, then $\bar{D}^{\prime \prime}=\left(C_{\infty},-\log |w|^{2}+\log \max \left\{\beta^{2}|w|^{2}, \alpha^{2}\right\}\right)$, where $w=y / x$. Thus, as in (2), we can see that the positive part of $\bar{D}^{\prime \prime}$ is

$$
\left(\theta^{\prime} C_{\infty},-\theta^{\prime} \log |w|^{2}+\log \max \left\{\beta^{2}|w|^{2 \theta^{\prime}}, 1\right\}\right),
$$

where $\theta^{\prime}=\log \beta /(\log \beta-\log \alpha)$, so that the positive part of $\bar{D}=\bar{D}^{\prime \prime}+\widehat{(z)}$ is

$$
\left(C_{0}-\left(1-\theta^{\prime}\right) C_{\infty},-\log |z|^{2}+\log \max \left\{|z|^{2 \theta^{\prime}}, \beta^{2}\right\}\right)
$$

by Proposition 9.3.1.

Remark 9.4.2. Let us choose $\alpha, \alpha^{\prime}, \beta, \beta^{\prime} \in \mathbb{R}_{>0}$ such that $\alpha \geq 1, \alpha^{\prime} \geq 1, \alpha \beta^{\prime}<1$ and $\alpha^{\prime} \beta<1$. We set

$$
M=C_{0}+C_{\infty}, \quad \varphi=-\log |z|^{2}+\log \max \left\{\alpha^{2}|z|^{2}, \beta^{2}\right\}+\log \max \left\{\alpha^{\prime 2}, \beta^{\prime 2}|z|^{2}\right\}
$$

and $\bar{M}=(M, \varphi)$, that is,

$$
\varphi= \begin{cases}-\log |z|^{2}+\log \left(\alpha^{\prime} \beta\right)^{2} & \text { if }|z| \leq \beta / \alpha, \\ \log \left(\alpha \alpha^{\prime}\right)^{2} & \text { if } \beta / \alpha \leq|z| \leq \alpha^{\prime} / \beta^{\prime}, \\ \log |z|^{2}+\log \left(\alpha \beta^{\prime}\right)^{2} & \text { if }|z| \geq \alpha^{\prime} / \beta^{\prime} .\end{cases}
$$

It is easy to see that $\bar{M}$ is an effective arithmetic Cartier divisor of $\left(C^{0} \cap \mathrm{PSH}\right)$-type and that

$$
\widehat{\operatorname{deg}}\left(\left.\bar{M}\right|_{C_{0}}\right)=\log \left(\alpha^{\prime} \beta\right) \text { and } \widehat{\operatorname{deg}}\left(\left.\bar{M}\right|_{C_{\infty}}\right)=\log \left(\alpha \beta^{\prime}\right) .
$$

If we set

$$
\vartheta=\frac{\log \alpha+\log \alpha^{\prime}}{\log \alpha-\log \beta}, \quad \vartheta^{\prime}=\frac{\log \alpha+\log \alpha^{\prime}}{\log \alpha^{\prime}-\log \beta^{\prime}}
$$

and

$$
\psi=-\vartheta \log |z|^{2}+\log \max \left\{\alpha^{2}|z|^{2 \vartheta}, \alpha^{\prime-2}\right\}+\log \max \left\{\alpha^{\prime 2}, \alpha^{-2}|z|^{2 \vartheta^{\prime}}\right\},
$$


that is,

$$
\psi= \begin{cases}-\vartheta \log |z|^{2} & \text { if }|z| \leq \beta / \alpha, \\ \log \left(\alpha \alpha^{\prime}\right)^{2} & \text { if } \beta / \alpha \leq|z| \leq \alpha^{\prime} / \beta^{\prime}, \\ \vartheta^{\prime} \log |z|^{2} & \text { if }|z| \geq \alpha^{\prime} / \beta^{\prime},\end{cases}
$$

then the positive part of $\bar{M}$ is

$$
\left(\vartheta C_{0}+\vartheta^{\prime} C_{\infty}, \psi\right)
$$

This can be checked in a similar way to Proposition 9.4.1. The details are left to the readers. In the case where $\alpha=\alpha^{\prime}=1$, the negative part of $\bar{M}$ is $\bar{M}$ itself, which means that the support of the negative part contains $C_{0}$ and $C_{\infty}$ despite $C_{0}-C_{\infty}=(z)$. This example also shows that if the positive parts of $\bar{D}$ and $\bar{D}^{\prime}$ are $\bar{P}$ and $\bar{P}^{\prime}$ respectively, then the positive part of $\bar{D}+\bar{D}^{\prime}$ is not necessarily $\bar{P}+\bar{P}^{\prime}$.

Remark 9.4.3. Let $\lambda$ be a positive real number. We set

$$
\phi_{\lambda}=-\log |z|^{2}+\log \left(|z|^{2}+\lambda\right) \quad \text { and } \quad \bar{M}_{\lambda}=\left(C_{0}, \phi_{\lambda}\right) .
$$

We denote $\bar{M}_{1}$ by $\bar{L}$, that is, $\bar{L}=\left(C_{0},-\log |z|^{2}+\log \left(|z|^{2}+1\right)\right)$. It is easy to see that $\bar{M}_{\lambda}$ is an arithmetic Cartier divisor of $\left(C^{\infty} \cap \mathrm{PSH}\right)$-type, $\widehat{\operatorname{deg}}\left(\bar{M}_{\lambda}^{2}\right)=(\log \lambda+1) / 2$ and that $\bar{M}_{\lambda}$ is nef for $\lambda \geq 1$. In particular, $\bar{M}_{\lambda}$ is big for $\lambda \geq 1$.

From now on, we fix $\lambda$ with $0<\lambda<1$. By using the inequality

$$
\log (1+\lambda x) \geq \lambda \log (1+x) \quad\left(x \in \mathbb{R}_{\geq 0}\right),
$$

we can see that $\lambda \bar{L} \leq \bar{M}_{\lambda}$, which means that $\bar{M}_{\lambda}$ is big. On the other hand,

$$
\widehat{\operatorname{deg}}\left(\left.\bar{M}_{\lambda}\right|_{C_{0}}\right)=\log \lambda<0,
$$

so that $\bar{M}_{\lambda}$ is not nef. We set

$$
\Phi_{\lambda}=d d^{c}\left(\log \left(|z|^{2}+\lambda\right)\right)=\frac{\lambda}{2 \pi \sqrt{-1}\left(|z|^{2}+\lambda\right)^{2}} d z \wedge d \bar{z}
$$

which is an $F_{\infty}$-invariant volume form on $\mathbb{P}^{1}(\mathbb{C})$ with $\int_{\mathbb{P}^{1}(\mathbb{C})} \Phi_{\lambda}=1$. Moreover, we set

$$
\begin{aligned}
& \widehat{\operatorname{Div}}_{\Phi_{\lambda}}\left(\mathbb{P}_{\mathbb{Z}}^{1}\right)_{\mathbb{R}} \\
& =\left\{\begin{array}{l|l}
\left(A, g_{A}\right) & \begin{array}{l}
(1) A \text { is an } \mathbb{R} \text {-Cartier divisor on } \mathbb{P}_{\mathbb{Z}}^{1} \\
(2) g_{A} \text { is an } F_{\infty} \text {-invariant } A \text {-Green function of } C^{\infty} \text {-type } \\
\text { on } \mathbb{P}^{1}(\mathbb{C}) \text { such that } d d^{c}\left(\left[g_{A}\right]\right)+\delta_{A}=(\operatorname{deg}(A)) \Phi_{\lambda}
\end{array}
\end{array}\right\},
\end{aligned}
$$


which is the Arakelov Chow group consisting of admissible metrics with respect to $\Phi_{\lambda}$ due to Arakelov-Faltings [7]. Let us check that the set

$$
\left\{\left(A, g_{A}\right) \in \widehat{\operatorname{Div}}_{\Phi_{\lambda}}\left(\mathbb{P}_{\mathbb{Z}}^{1}\right)_{\mathbb{R}} \mid\left(A, g_{A}\right) \text { is nef and }(0,0) \leq\left(A, g_{A}\right) \leq \bar{M}_{\lambda}\right\}
$$

has only one element $(0,0)$.

Indeed, let $\bar{A}=\left(A, g_{A}\right)$ be an element of the above set. Then there are constants $a, b$ such that $0 \leq a \leq 1$ and $\bar{A}=a \bar{M}_{\lambda}+(0, b)$. Since $g_{A} \leq \phi_{\lambda}$, we have $b \leq(1-a) \phi_{\lambda}$. Thus $b \leq 0$ because $\phi_{\lambda}(\infty)=0$. In addition,

$$
\widehat{\operatorname{deg}}\left(\left.\bar{A}\right|_{C_{0}}\right)=a \log \lambda+b \geq 0 .
$$

In particular, $b \geq 0$, so that $b=0$, and hence $a \log \lambda \geq 0$. Thus $a=0$.

This example shows that the Arakelov Chow group consisting of admissible metrics is insufficient to get the Zariski decomposition.

Finally note that $\lambda \bar{L}$ is not necessarily the positive part of $\bar{M}_{\lambda}$ because $\widehat{\operatorname{vol}}\left(\bar{M}_{\lambda}\right) \geq(\log \lambda+1) / 2$ (cf. Theorem 6.6.1), $\widehat{\operatorname{vol}}(\lambda \bar{L})=\lambda^{2} / 2$ and $(\log \lambda+1) / 2>$ $\lambda^{2} / 2$ for $0<1-\lambda \ll 1$.

Remark 9.4.4. Let $n$ be a positive integer and $f \in \mathbb{R}[T]$ such that $\operatorname{deg}(f)=2 n$ and $f(t)>0$ for all $t \in \mathbb{R}_{\geq 0}$. It seems not to be easy to find the positive part of

$$
\left(n C_{0},-n \log |z|^{2}+\log f(|z|)\right)
$$

on $\mathbb{P}_{\mathbb{Z}}^{1}$.

\section{Acknowledgments}

I would like to thank Prof. Bauer, Prof. Caibăr and Prof. Kennedy for sending me their wonderful paper concerning Zariski decompositions in vector spaces; our research was done independently. I also express my hearty thanks to Prof. Yuan and the referee for their questions and comments.

This research was partially supported by the Ministry of Education, Science, Sports and Culture, Grant-in-Aid for Scientific Research (A), 22244003, 2012.

\section{References}

[1] T. Bauer, A simple proof for the existence of Zariski decompositions on surfaces, J. Algebraic Geom. 18 (2009), 789-793. Zbl 1184.14007 MR 2524598

[2] T. Bauer, M. Caibăr and G. Kennedy, Zariski decomposition: A new (old) chapter of linear algebra, Amer. Math. Monthly 119 (2012), 25-41. Zbl pre06071087 MR 2877664

[3] R. Berman and J.-P. Demailly, Regularity of plurisubharmonic upper envelopes in big cohomology classes, in Perspectives in analysis, geometry, and topology: on the occasion of the 60th birthday of Oleg Viro, Progr. Math. 296, Birkhäuser, 2012, 39-66. MR 2884031 
[4] Z. Błocki and S. Kołodziej, On regularization of plurisubharmonic functions on manifolds, Proc. Amer. Math. Soc. 135 (2007), 2089-2093. Zbl 1116.32024 MR 2299485

[5] H. Chen, Positive degree and arithmetic bigness, arXiv:0803.2583 [math.AG].

[6] _ Arithmetic Fujita approximation, Ann. Sci. École Norm. Sup. 43 (2010), 555-578. Zbl 1202.14024 MR 2722508

[7] G. Faltings, Calculus on arithmetic surfaces, Ann. of Math. 119 (1984), 387-424. Zbl 0559.14005 MR 0740897

[8] L. Hörmander, Notions of convexity, Progr. Math. 127, Birkhäuser, 1994. Zbl 0835.32001 MR 1301332

[9] M. Klimek, Pluripotential theory, London Math. Soc. Monogr. 6, Oxford Univ. Press, 1991. Zbl 0742.31001 MR 1150978

[10] R. Lazarsfeld, Positivity in algebraic geometry I, II, Springer, 2004. Zbl 1093.14501(I) Zbl 1093.14500(II) MR 2095471(I) MR 2095472(II)

[11] V. Maillot, Géométrie d'Arakelov des variétés toriques et fibrés en droites intégrables, Mém. Soc. Math. France 80 (2000). Z Zbl 0963.14009 MR 1775582

[12] H. Matsumura, Commutative ring theory, Cambridge Stud. Adv. Math. 8, Cambridge Univ. Press, 1989. Zbl 0666.13002 MR 1011461

[13] A. Moriwaki, Arithmetic height functions over finitely generated fields, Invent. Math. 140 (2000), 101-142. Zbl 1007.11042 MR 1779799

[14] Continuity of volumes on arithmetic varieties, J. Algebraic Geom. 18 (2009), 407457. Zbl 1167.14014 MR 2496453

[15] _ Continuous extension of arithmetic volumes, Int. Math. Res. Notices 2009, 35983638. Zbl 1192.14022 MR 2539186

[16] Estimation of arithmetic linear series, Kyoto J. Math. 50 (2010), 685-725. Zbl 1210.14028 MR 2740691

[17] MR 2823999

[18] _ Toward Dirichlet's unit theorem on arithmetic varieties, Kyoto J. Math., to appear; arXiv:1010.1599v2 [math.AG].

[19] $\_$Arithmetic linear series with base conditions, Math. Z., DOI 10.1007/s00209-0120991-2.

[20] Characterization of nef arithmetic divisors on arithmetic surfaces, arXiv:1201.6124 [math.AG].

[21] N. Nakayama, Zariski-decomposition and abundance, MSJ Memoirs 14, Math. Soc. Japan, Tokyo, 2004. Zbl 1061.14018 MR 2104208

[22] R. Webster, Convexity, Oxford Univ. Press, 1994. Zbl 0835.52001 MR 1443208

[23] X. Yuan, On volumes of arithmetic line bundles, Compos. Math. 145 (2009), 1447-1464. Zbl 1197.14023 MR 2575090

[24] O. Zariski, The theorem of Riemann-Roch for high multiples of an effective divisor on an algebraic surface, Ann. of Math. 76 (1962), 560-615. Z Zbl 0124.37001 MR 0141668

[25] S. Zhang, Positive line bundles on arithmetic varieties, J. Amer. Math. Soc. 8 (1995), 187221. Zbl 0861.14018 MR 1254133

[26] Small points and adelic metrics, J. Algebraic Geom. 4 (1995), 281-300. Zbl 0861.14019 MR 1311351 